\title{
DISSERTATION
}

\section{DESIGN OF A MULTI-SENSOR PLATFORM FOR INTEGRATING EXTRACELLULAR ACIDIFICATION RATE WITH MULTI-METABOLITE FLUX MEASUREMENT FOR SMALL BIOLOGICAL SAMPLES}

\author{
Submitted by \\ Yusra M Obeidat \\ Department of Electrical and Computer Engineering
}

In partial fulfillment of the requirements

For the Degree of Doctor of Philosophy

Colorado State University

Fort Collins, Colorado

Spring 2019

Doctoral Committee:

Advisor: Tom Chen

Sudeep Pasricha

George Collins

Stuart Tobet 
Copyright by Yusra M Obeidat 2019

All Rights Reserved 


\section{ABSTRACT}

\section{DESIGN OF A MULTI-SENSOR PLATFORM FOR INTEGRATING EXTRACELLULAR ACIDIFICATION RATE WITH MULTI-METABOLITE FLUX MEASUREMENT FOR SMALL BIOLOGICAL SAMPLES}

Cell metabolism involves a set of cellular chemical reactions that are very important to cell development as well as its response to environmental changes around the cell. Understanding cell metabolism and the associated metabolic pathways has been the focus of many research efforts and it is gaining more attention recently. In assisted reproductive technology (ART), understanding metabolism of oocytes and embryos provides the possibility of selecting more viable embryos for transfer and reducing the number of embryos transferred in a given in vitro fertilization (IVF) cycle. Although stage-specific morphologic markers and grading systems have been developed and widely in use, this approach is unable to reliably assess the physiological status of the embryo and it is not only subjective but has a poor correlation with subsequent developmental competence. Therefore, there is an ever-increasing need for noninvasive quantitative markers of embryo viability. Analysis of metabolism has proved to be a valuable marker of embryo viability based on animal models. Through noninvasive analysis of metabolic markers, it will be feasible to identify those embryos with the highest probability of establishing a healthy pregnancy.

Crucial to cell metabolic process is a set of analytes that can be used as indicators of cell metabolism. They include oxygen, glucose, and lactate. Rates of cellular oxygen consumption (OCR) and extracellular acidification (ECAR) are widely used proxies for mitochondrial oxidative phosphorylation (OXPHOS) and glycolytic rate in cell metabolism studies. However, ECAR can result from both oxidative metabolism (carbonic acid formation) and glycolysis (lactate release), potentially leading to erroneous conclusions about metabolic substrate utilization. Co- 
measurement of extracellular glucose and lactate flux along with OCR and ECAR can improve the accuracy and provide better insight into cellular metabolic processes but is currently not feasible with any commercially available instrumentation.

Some techniques for measuring dissolved oxygen (DO), glucose and lactate rely on fluorescent labels. These techniques are incredibly labor intensive, and the pipet construction used is complex comparing with solid state and electrochemical methods. Injecting a cell with fluorescent label can also lead to experimental error, since biochemical mechanisms inside of the cell may interact with the label. Other techniques include the use of scanning electrochemical microscopy (SECM) for studying the metabolism of single cells, but it has its drawbacks including probe fouling, complex instrumentation, as well as calibration can also be challenging. Furthermore, electrochemical microphysiometers were used for monitoring changes in glucose and lactate concentrations in cell cultures, but these techniques need larger sample volumes and might need difficult calibration.

Due to the lack of quantitative and real-time monitoring of cell metabolism, the success rate of in-vitro fertilization (IVF) is still low, with very low percentage of embryos transferred resulting in a term pregnancy [1-2]. Therefore, more work needs to be done for testing embryos metabolism in-vitro to improve the culture conditions and reduce the effect of environmental stresses and chose the media that balance all nutrients the cell needs during development.

In this work, we present a miniaturized multi-sensor platform capable of real-time monitoring of OCR and ECAR along with extracellular lactate and glucose flux for small biological samples such as single equine and bovine embryos. This multiplexed approach enables validation of ECAR resulting from OXPHOS versus glycolysis, and expression of metabolic flux ratios that provide further insight into cellular substrate utilization. We demonstrate expected shifts in embryo metabolism during development and in response to OXPHOS inhibition as a model system for monitoring metabolic plasticity in very small biological samples. 
In this work, DO was measured amperometrically using a three-electrode system of working $(\mathrm{WE})$, counter (CE) and reference (RE) electrodes. Glucose and lactate were measured enzymatically by measuring the current generated from the oxidation of hydrogen peroxide generated from the catalysis of glucose or lactate at the WEs with their catalysis enzymes. $\mathrm{pH}$ was measured potentiometrically using two electrodes system of Indium Tin Oxide (ITO) WE and Au pseudo RE. A micro-chamber containing all four sensors was designed and manufactured to investigate single cell immersed in a respiration medium. The micro-chamber design is an important part of the platform that provides sufficient change of the target analytes in the microenvironment that enables the sensors to measure tiny changes of the target analytes due to cell respiration. This setup helps to measure the analytes with a change in concentration ranges from (0.001 to 30$) \mathrm{fmol} / \mathrm{s}$ with high specificity which is comparable with what was published in literature. The specificity of our sensors was clearly determined by monitoring the switch in metabolism to glycolysis induced by adding oligomycin as an inhibitor for ATP-synthase. The ability to measure the extracellular acidification rate (ECAR) in addition to lactate production can help to differentiate the respiratory acid production from glycolytic acidification. The ability of the sensor to detect a metabolic shift from oxidative phosphorylation (OXPHOS) to glycolysis was demonstrated in embryos by an ablation of oxygen consumption and an increase in lactate production as well as ECAR following addition of oligomycin. The increase in $\mathrm{pH}$ change rate after adding oligomycin and its slowdown after FCCP further indicates the dependence of cell on glycolysis and the increase of lactate production. The results of bovine or equine embryos show that the embryos metabolism change with development as expected and the amounts of glucose and oxygen uptakes and lactate production increase at later stages of developments, which match the existing biological knowledge of increasing the need for ATP production at later stages of development.

Our system is capable to provide single-cell metabolism measurement with more complete panel than what commercially available devices such as Seahorse provides. Our results provide a clear insight into the mechanism of OXPHOS and glycolysis for single cells and a more complete 
analysis to include inter-sensor interference for improved accuracy. The capability of the platform is illustrated with measurements multi-metabolites of single-cell equine or bovine embryos for assisted reproduction technologies. However, this platform has a wide potential utility for analyzing small biological samples such as single cells and tumor biopsies for immunology and cancer research applications. Furthermore, we also present a preliminary interference analysis of the multi-sensor platform to allow better understanding of sensor interference in the proposed multi-sensor platform. 


\section{ACKNOWLEDGEMENTS}

First and above all, I praise God, the almighty for providing me this opportunity and granting me the capability to proceed successfully.

I would like to express my special deep gratitude to professor Tom Chen, my research advisor, for his patient guidance, enthusiastic encouragement and useful critiques of this research work.

I would like to extend my thanks and appreciation to doctor Elaine Carnevale and doctor Adam Chicco for their support, contribution in understanding and discussing a lot of information that were valuable and very helpful in my research work.

I would like to thank August DeMann and Stuart Field for their support in metal evaporation.

I would like to thank all my lab members for their support by providing either valuable suggestions, ideas or technical assistance.

My grateful thanks are also extended to Marwan Obeidat and all my friends and colleagues at Colorado State University.

Finally, I can't finish without thanking my great family. I warmly thank and appreciate my parents for their prayers, material and spiritual support in all aspects of my life. 


\section{TABLE OF CONTENTS}

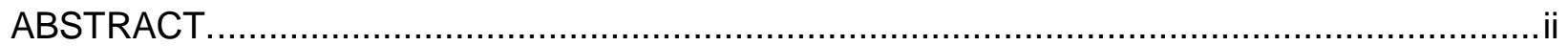

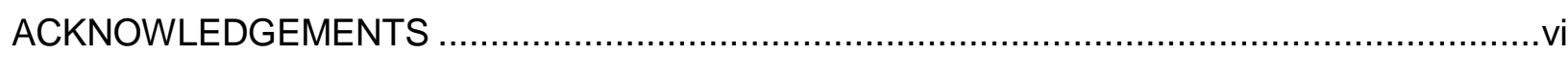

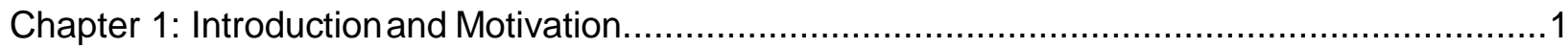

Chapter 2: Background and Existing Research Work ...................................................

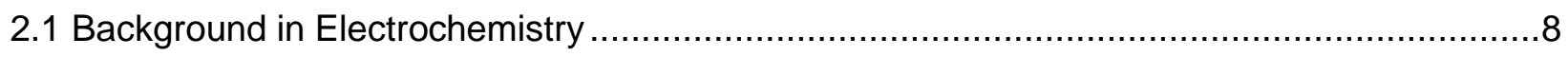

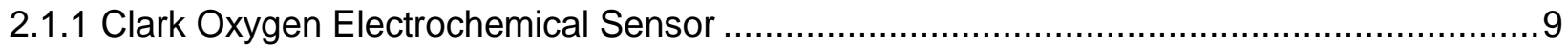

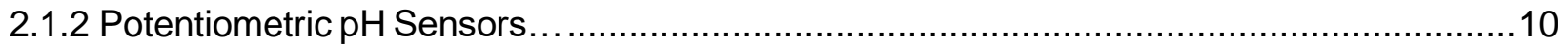

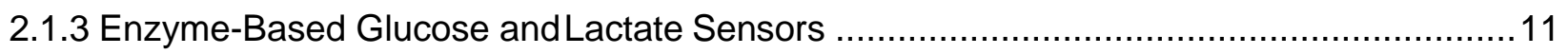

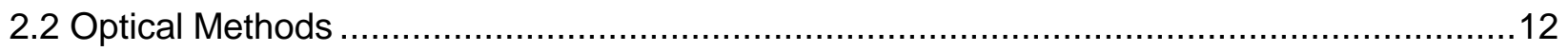

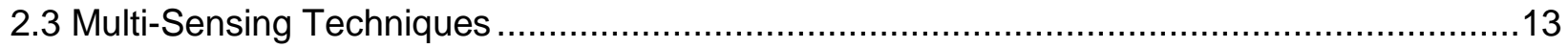

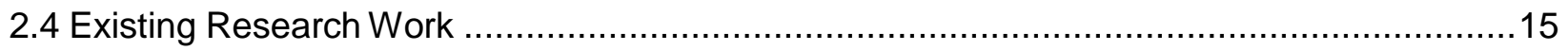

Chapter 3: Characterization of an $\mathrm{O}_{2}$ Sensor Using Microelectrodes ....................................19

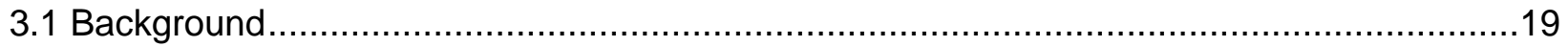

$3.2 \mathrm{O}_{2}$ Sensor Design and Characterization ............................................................. 19

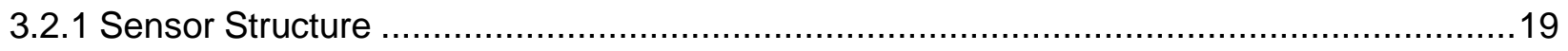

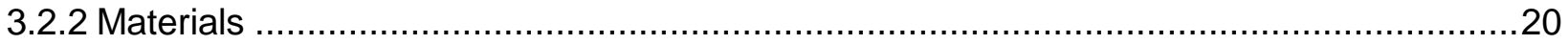

3.2.3 Sensor Surface Modification andOperating Conditions.............................................20

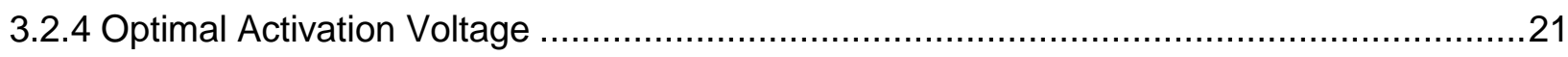

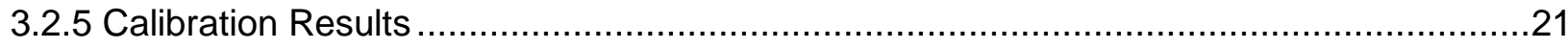

Chapter 4: Monitoring Oocyte/Embryo $\mathrm{O}_{2}$ Respiration Using Three Electrodes Clark Sensor .....23

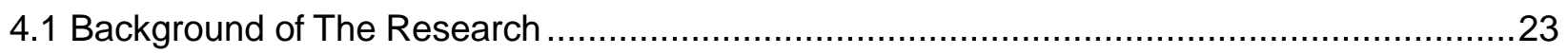

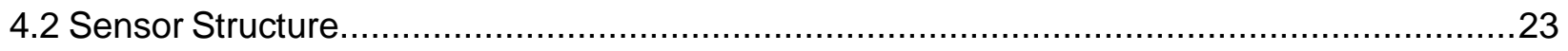

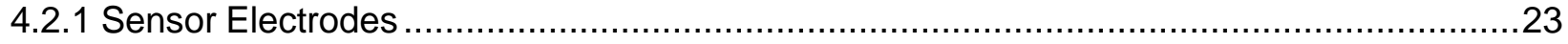

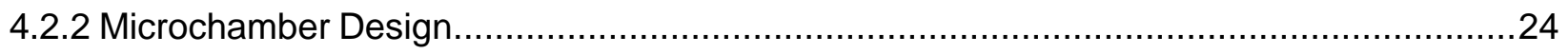

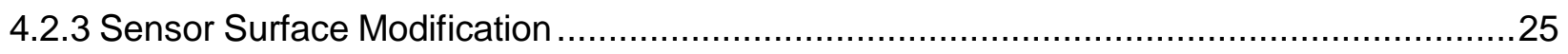

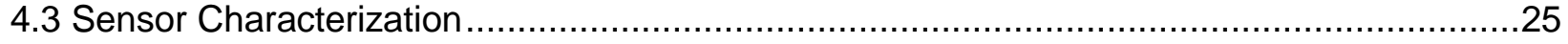

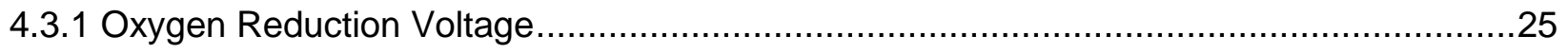

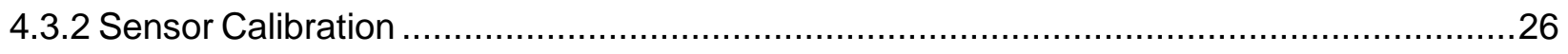

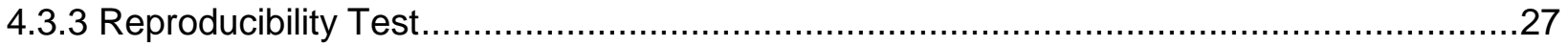


4.3.4 Verification of Airtightness of the Micro-Chamber

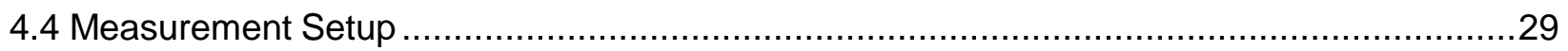

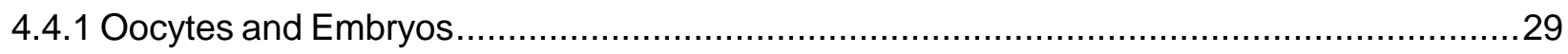

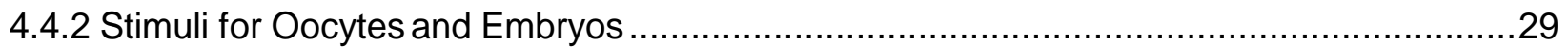

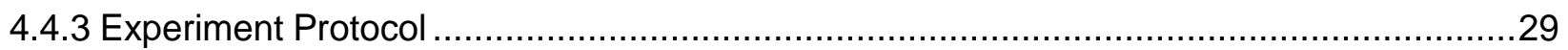

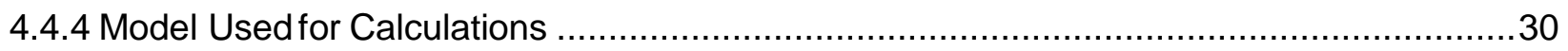

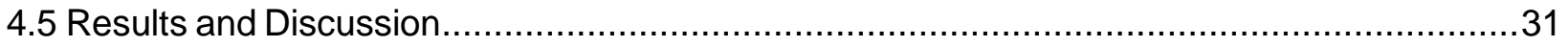

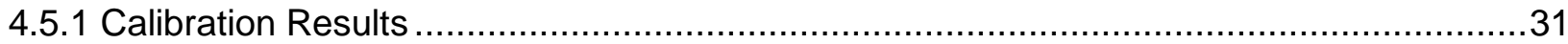

4.5.2 Measurement of Basal Mitochondrial Respiration in Individual Equine Oocytes...............32

4.5.3 Effect of Oligomycin Addition on Cell Respiration of Equine Oocytes .............................33

4.5.4 Effect of FCCP Titration on Cell Respiration of Bovine Oocytes and Embryos .................35

4.5.5 Basal Respiration and the Effect of Oligomycin and FCCP Titration on Bovine Oocytes .....36

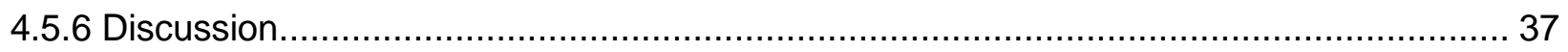

Chapter 5: A multi-Sensor System for Measuring Bovine Embryo Metabolism.......................39

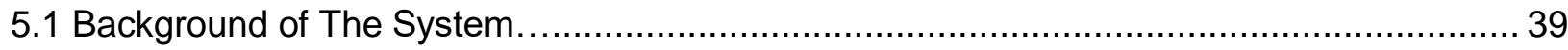

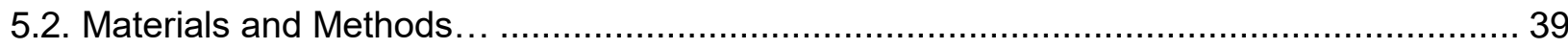

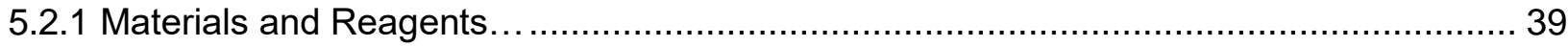

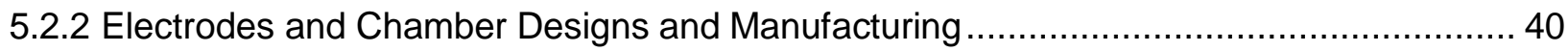

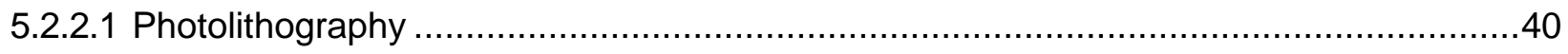

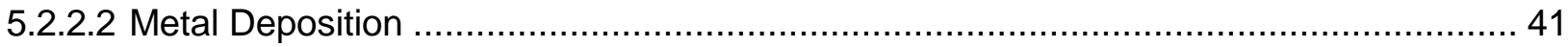

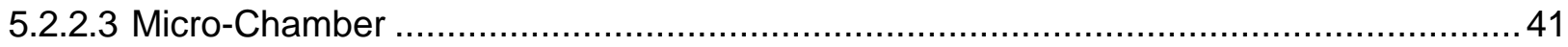

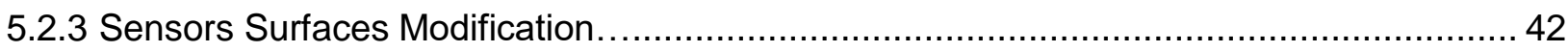

5.2.3.1 Oxygen Electrode Surface Modification............................................................ 42

5.2.3.2 Glucose Electrode Surface Modification......................................................... 42

5.2.3.3 Lactate Electrode Surface Modification............................................................ 42

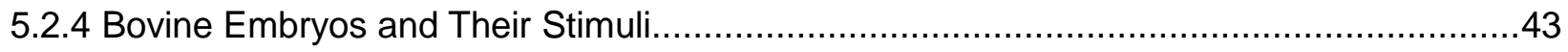

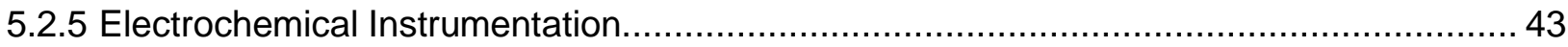

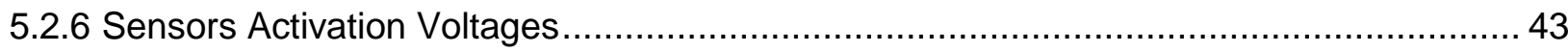

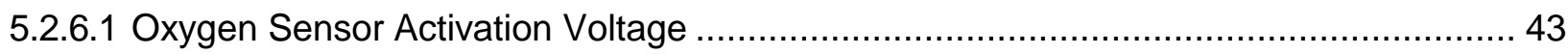

5.2.6.2 Glucose Sensor Activation Voltage ................................................................... 44

5.2.6.3 Lactate Sensor Activation Voltage .............................................................. 44 
5.2.6.4 Hydrogen peroxide $\left(\mathrm{H}_{2} \mathrm{O}_{2}\right)$ Activation Voltage .......................................................44

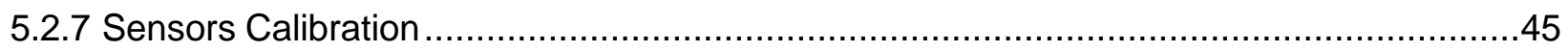

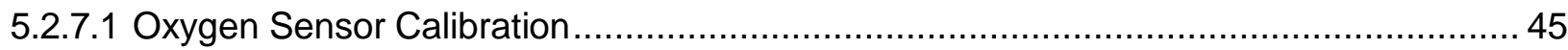

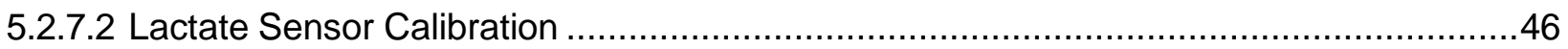

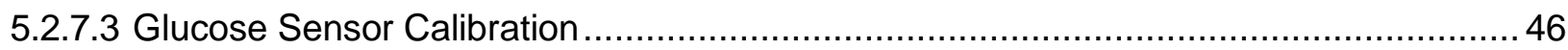

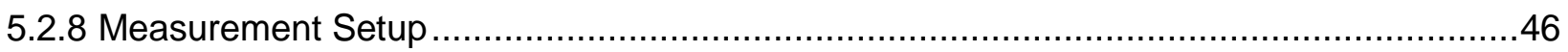

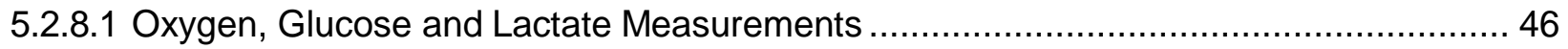

5.2.9 Model Used for Analyte Consumption/Production Calculations....................................47

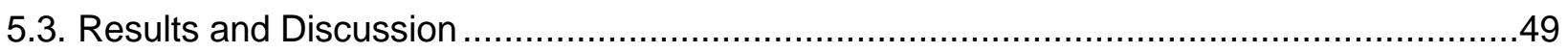

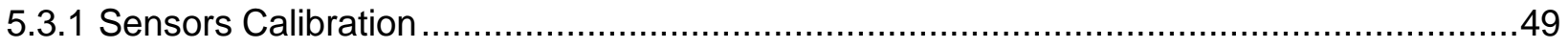

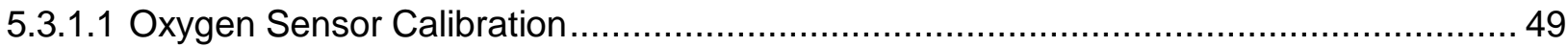

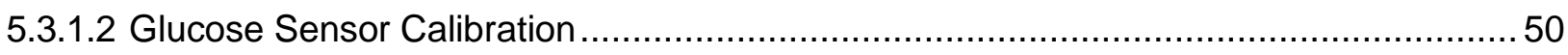

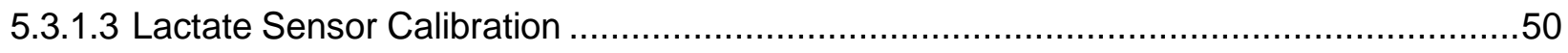

5.3.2 Characterization of Bovine Embryo Energy Metabolism ........................................... 50

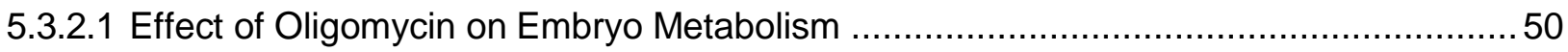

5.3.2.2 Evaluation of Embryo Metabolism Throughout Development..................................... 51

5.3.2.3 Metabolic Flux Ratios Reveal Shifts in Glucose Utilization during Embryo

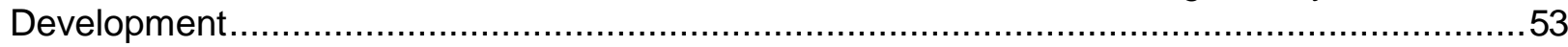

5.3.3 Advantages of the Present Design over Other Metabolism Sensing Systems ...................54

Chapter 6: Design of A Multi-Sensor Platform for Integrating Extracellular Acidification Rate with Multi-Metabolite Flux Measurement for Small Biological Samples

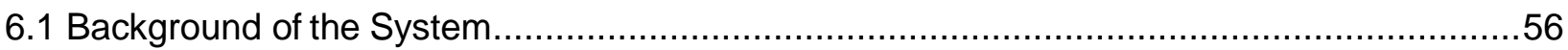

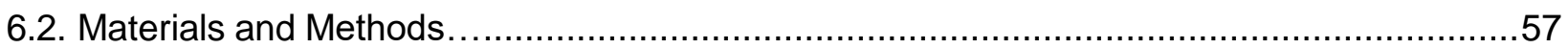

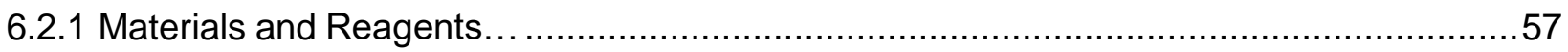

6.2.2 Electrodes and Chamber Designs and Manufacturing …..........................................57

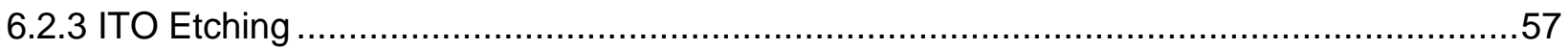

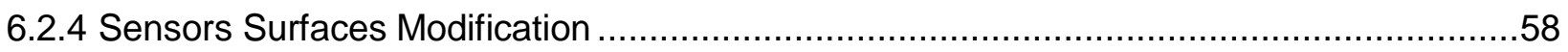

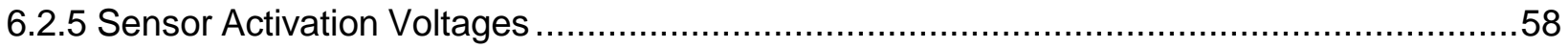

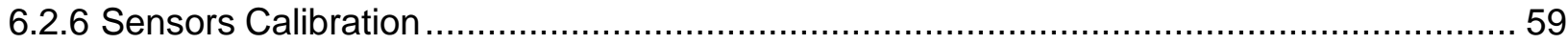

6.2.6.1 Calibration for Oxygen, Glucose and Lactate Sensors ……....................................59

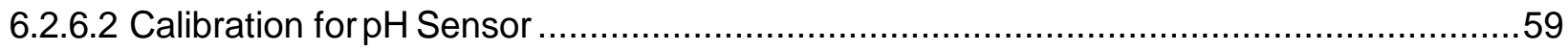




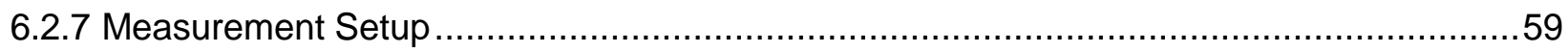

6.2.7.1 Oxygen, Glucose, Lactate and pH Measurements ...................................................59

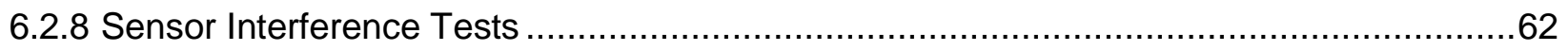

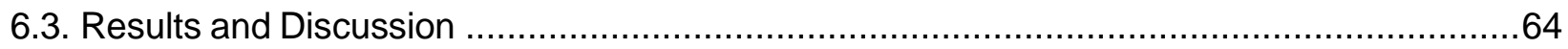

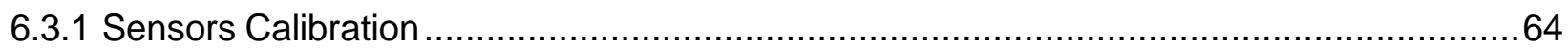

6.3.1.1 Oxygen, Glucose and Lactate Sensors Calibration Results ........................................64

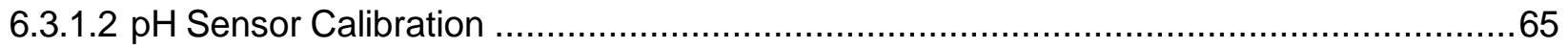

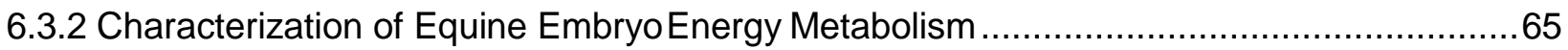

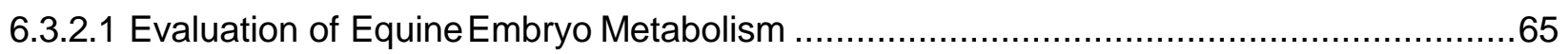

6.3.2.2 Effect of Oligomycin and FCCP Titration on Embryo Metabolism ..................................70

6.3.2.3 Effect of Changing pH on Glucose and Lactate Measurements ....................................73

6.3.3 Sensor Interference between Glucose and Lactate Sensors .........................................73

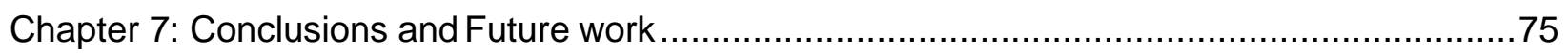

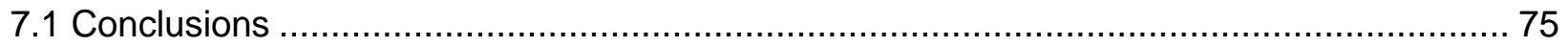

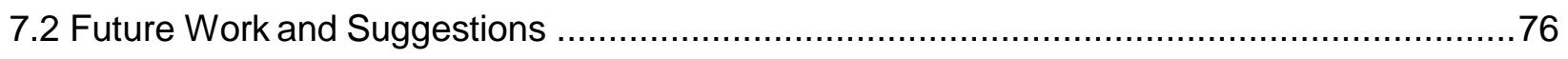

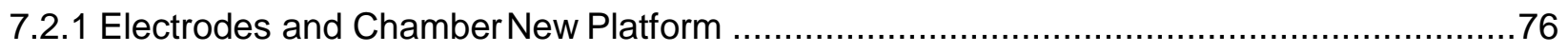

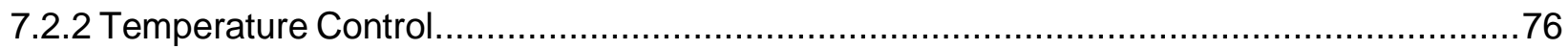

7.2.3 Design of Fully Controlled Circuit to Replace Benchtop …..........................................77

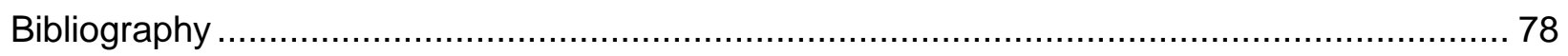

Appendix A: Modifying enzymes contents to reduce toxicity on cells... ................................ 86

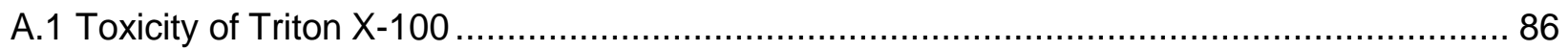

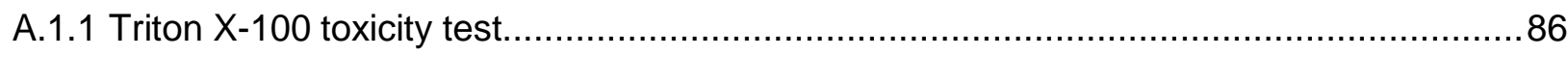

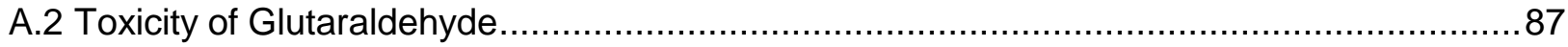

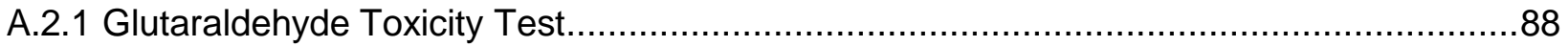

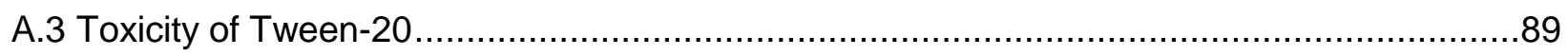

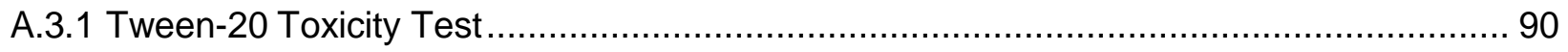

A.4 Conclusion of the Toxicity Tests and Enzymes Modifications.......................................92

Appendix B: Bovine Embryos Grading, Experiment Setup, Statistical Analysis and Sensors

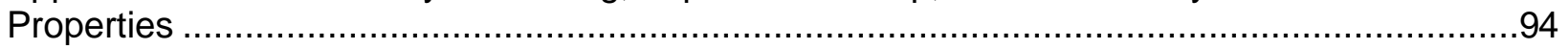

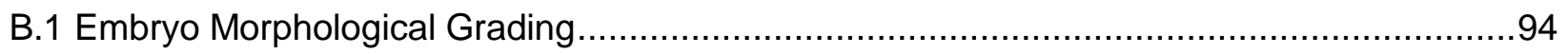

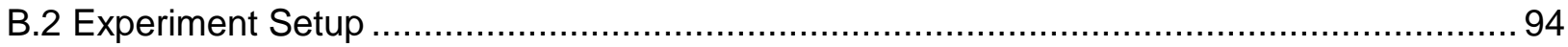

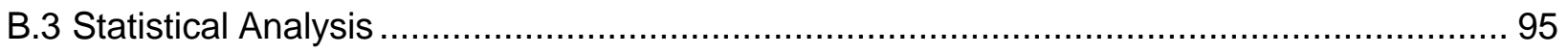




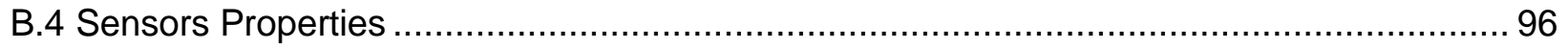

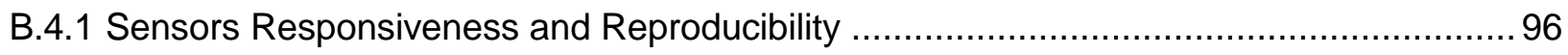

B.4.1.1 Oxygen Sensor Responsiveness and Reproducibility …………........................... 96

B.4.1.2 Glucose Sensor Responsiveness and Reproducibility ......................................... 97

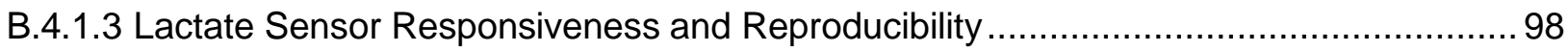

B.4.2 The Use of Quasi-Reference Electrode …………….......................................... 99

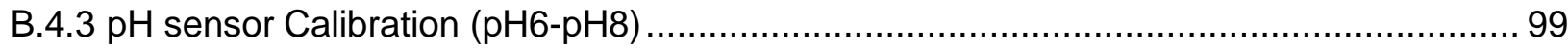

Appendix C: Examples of the measured data...........................................................101

C.1 Bovine Embryos Metabolism: Basal vs Glycolysis................................................. 101

C.2 Equine Embryos Metabolism (Basal, Oligomycin, FCCP ${ }_{1-3}$ ) ...................................... 102

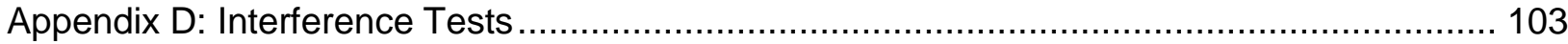

D.1 Effect of Enzymes Reactions on $\mathrm{O}_{2}$ Level in Multi-Sensor Platform ...............................103

D.2 Interference between Enzyme Sensors in Multi-Sensor System ................................... 106

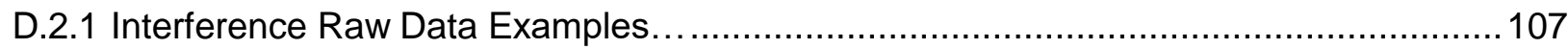

Appendix E: Effect of the Platform on Embryos Development ............................................. 108

Appendix F: Matlab Gui........................................................................................111

Appendix G: Publications......................................................................................... 140 


\section{Chapter 1: Introduction and Motivation}

Cellular metabolism is the set of chemical reactions that generate chemical energy for the cells for their daily activities and produce complex molecules to maintain the cell life and support its reproduction [3]. Metabolism involves complex sequences of controlled biochemical reactions known as metabolic pathways, in which one chemical is transformed into another by a sequence of enzymes [4].

Chemical energy is mainly derived from a molecule called adenosine triphosphate (ATP). ATP forms adenosine diphosphate (ADP) through hydrolysis process, ADP is an inorganic phosphate molecule, and energy [3]. Metabolic pathways are generally devided into two pathways: a catabolic pathway which includes any metabolic function associated with making ATP, and an anabolic pathway which includes any metabolic function associated with assembling more complex molecules from carbon-containing molecules produced out of the catabolic pathway. The new molecules built via anabolic pathways are useful for building cell structures and maintaining the cell [3-4]. The catabolic pathway is dominated by the breakdown of glucose into a form that releases sufficient energy to make ATP and it is simply the transfer of energy stored in glucose bonds to phosphate bonds. The transfer of electrons from glucose to oxygen results in a release of energy that is sufficient to convert (phosphorylate) ADP molecules to ATP molecules and if glucose were to transfer electrons directly to oxygen, the release of energy would be so sudden that a large amount of heat would be produced, which would cause cell damage and would be extremely inefficient $[3,5]$. Therefore, a series of intermediate reactions are requiered to produce a similar amount of energy and produce ATP without causing cell damage. The first intermediate reaction that glucose undergoes is glycolysis, where glucose sugar is broken into a compound called pyruvate. Pyruvate is used to initiate a series of chemical reactions known as the Krebs cycle. The Krebs cycle converts some ADP molecules to ATP and completes the conversion of glucose to carbon dioxide $\mathrm{CO}_{2}$. In an aerobic metabolic cycle, oxygen is the 
final electron acceptor [4-5]. Cellular respiration doesn't occur when oxygen is not readily available for accepting electrons at the end of the transport chain, and instead, electrons are transferred from glucose to some other organic, electron-accepting molecule. The process by which ATP is produced via electron transport from glucose to a non-oxygen molecule is called an anaerobic process and is essentially a secondary process for the cell to produce fuel in the absence of oxygen. The use of other electron acceptors (non-oxygen molecules) in the transport chain produces different byproducts than regular cellular respiration such as lactate. All these molecules are first broken down into pyruvate, which is then used in the Krebs cycle [3-4]. A schematic diagram that shows the major metabolic pathways in mamalian cells is shown in Fig. 1.1.

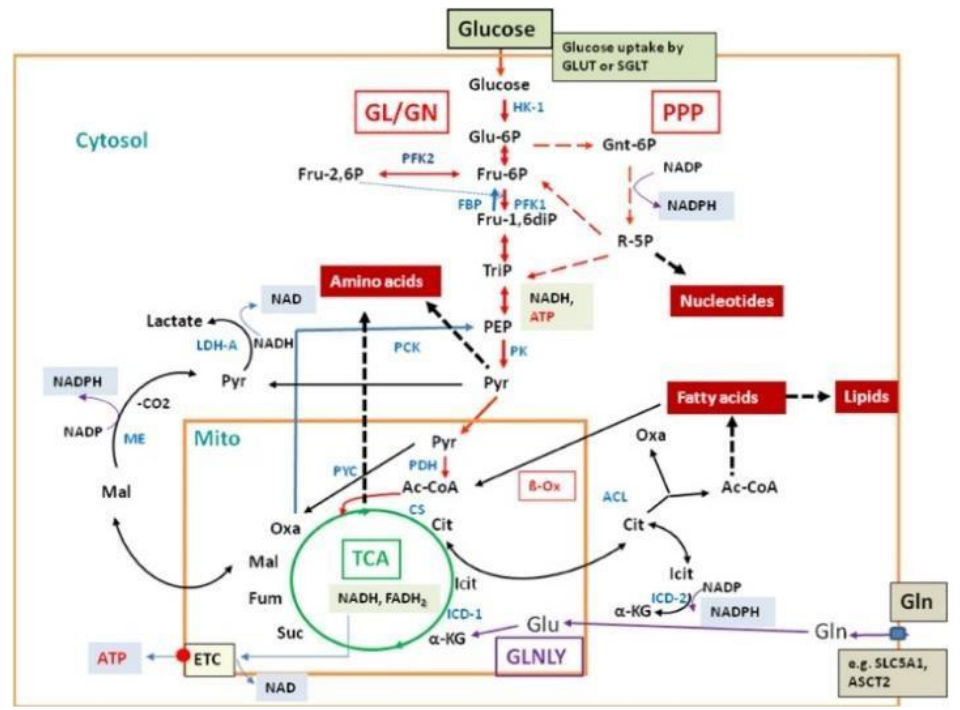

Fig.1.1. Major catabolic and anabolic pathways in mammalian cells. [3]

Metabolic activities of a cell are often associated with cell activity and viability, such as mitochondrial respiration [6]. Oxygen consumption of a cell can often be used as a good indicator of cell activity and viability that is closely associated with organismal metabolic health and disease [3, 5-6]. However, to better understand the cell's metabolic activity, it is desired to measure different metabolites from individual living cells [4, 7-12]. The development of sensing techniques to analyze at the single cell level is more desirable for biomedical and biological research. The 
most common analytes of interest at the single cell level, when anlyzing metabolism include inorganic molecules such as $\mathrm{DO}$ and $\mathrm{CO}_{2}$, organic molecules such as glucose, adenosine triphosphate (ATP), NADH, reduced flavin adenine dinucleotide (FADH ), and lactate. Combined with $\mathrm{pH}$, they are good indicators of cellular metabolic activity [4-14]. These analytes provide a good understanding of how well a cell is supporting proper metabolism through both catabolic and anabolic mean. For examples: Monitoring the amount of extracellular oxygen $\mathrm{O}_{2}$ give information about how well the cell can carry out respiration, extracellular $\mathrm{CO}_{2}$ reflects how well the Krebs cycle is being completed in the cell, extracellular glucose reflects the capacity for the cell to produce fuel and to carry out anabolic functions, and extracellular $\mathrm{pH}$ is an indication of cellular respiration, especially the production of $\mathrm{CO}_{2}$ and/or lactic acid [4].

Multiple analytes measurement provides a more complete understanding of cellular metabolic pathways and changes in these pathways in response to various chemical and biological stimulations. During the aerobic pathway the cells consume glucose and oxygen with $\mathrm{CO}_{2}$ as a product and in the anaerobic pathway the cells consume glucose in the absence of oxygen, producing lactic acid [3-5]. Therefore, a simplified view of cellular metabolism and more insight into both the aerobic and anaerobic pathways of the cells can be proposed by measuring analytes directly involved in the energy-producing pathways where the inputs are glucose and oxygen and the outputs are lactic acid and $\mathrm{CO}_{2}$ which both contribute to acidification (change in the extracellular $\mathrm{pH}$ level) [15]. By monitoring the direction and magnitude of the changes in these analytes, cellular metabolic responses to a particular agent can also be inferred.

Most studies at the present time focus on multiple cells measurements. Multiple-cell measurements are more compatible with the limitations of existing instrumentation technology. In order to develop a more complete understanding of inherently heterogeneous cell populations, it is necessary to measure and analyze a wide variety of parameters from individual living cells. Single-cell characteristics play an important role in determining population characteristics and the transient dynamics that lead to future cell expression and behavior at a population level $[4,7]$. 
Therefore, the need for a wide variety of sensing and measurement techniques to analyze at a single-cell level is increasing in the biological research community.

Dramatic variations in energetic metabolism can be experienced in cells based on their nature, activity, and microenvironment. Abnormal cellular bioenergetics are always associated with diseases such as obesity, diabetes, cancer, neurodegeneration and cardiomyopathy $[16,17]$ All phenotypic changes are driven by underlying adjustments of cellular bioenergetics which often have unique profiles $[18,19]$.

Cancer cells represent a good example of metabolic adaptation. Many cancer cells dispense completely with ATP generation through the highly efficient mitochondrial respiratory pathway and rely on glycolysis for ATP generation even when growing in the presence of oxygen. This process known as the Warburg effect $[20,21]$ and basically represents the increase in glucose uptake and the enhancement of glycolytic capacity and a high lactate production, along with the absence of respiration despite the presence of high oxygen concentration in tumor cells [22-24].

Living cells have relevant biological complexity and can be highly multiplexed to screen for drugs and their mechanisms. Therefore, measurement at cells level has become a favored format for drug discovery. Cells remodel bioenergetic pathways in response to a changing extracellular environment, disease and/or drug exposure, to drive phenotypic changes associated with these perturbations [25-28]. By measuring the changes in oxygen and extracellular acidification in the media surrounding cells, the relative state of aerobic and glycolytic metabolism can be determined, respectively [29-30].

The development of new techniques for measuring the cellular metabolites uptake or release has wide applications in exploring directed evolution, drug toxicity, and cancer [31-32]. Measuring the metabolism of single cells is very useful because it helps avoiding the complexities associated with cellular heterogeneity. Single cell sensitivity is necessary for studies of organisms composed of a single or small number of cells, such as embryos during early development. There is also a strong need in the clinical field of assisted reproduction to develop non-invasive methods for 
evaluating the health and developmental potential of embryos. The methods that have been developed and widely used for selecting embryos in clinical in vitro fertilization (IVF) programs are stage-specific morphologic markers and grading systems, these methods are based on physical characteristics identified through optical microscopy [1-2]. But these methods are unable to reliably assess the physiological status of the embryo and it is well recognized within the clinical IVF community that morphology is not only subjective but has a poor correlation with subsequent developmental competence [33]. As a result, multi-fetal pregnancies might happen because more than one embryo are often transferred to the uterus in an IVF cycle, which increase risks to the pregnancy, the mother, and the child [34]. Therefore, a variety of non-invasive approaches are being developed to assess embryonic developmental potential to move toward single embryo transfer [35].

Metabolism is considered as one of the most intensely investigated biologic processes in early embryonic development. There are increasing studies demonstrated that embryos with greater development capacity have distinct metabolic profiles [31, 32]. Many previous studies have demonstrated that the early embryo undergoes dramatic changes in its metabolism, switching from a low to a high basal metabolic rate [36]. The increase in metabolic activity indicates a switch in utilization of nutrients, switching from a pyruvate to a glucose-based metabolism [37]. Furthermore, energy metabolism may serve as a biomarker for the development of mammalian embryos and some alterations in metabolism have been demonstrated as a reason for abnormal embryonic development [38]. Non-invasively assessing metabolism of embryos most commonly means the ability to evaluate what embryos consume and produce through analysis of culture media. Measuring the consumption and production of several key energetic substrates such as glucose, lactate and pyruvate is one of the standard techniques of evaluating embryonic metabolism. Fluorometric enzymatic assays have been used in several studies to measure change in cell metabolites, specifically glucose, pyruvate, and lactate. The main drawbacks of this technology are: it is incredibly labor intensive and pipet construction is complex 
$[39,40]$. An array of alternative microfabricated approaches have been developed in recent years for studying cellular metabolism, many of them are technically more complex and may not be suitable for integration with culture systems or routine clinical use. One example is the use of electrochemical microphysiometers for monitoring changes glucose and lactate concentrations in cell cultures using both continuous [41] and discrete [8] fluid flow approaches that needs larger sample volumes and might need difficult calibration. Furthermore, scanning electrochemical microscopy (SECM) has been demonstrated as a noninvasive means for studying the metabolism of single cells by measuring oxygen [42] and glucose and lactate [43], but it has its drawbacks including probe fouling, complex instrumentation, as well as calibration can also be challenging.

These methods are technically complex and may not be suitable for routine clinical use. The drawbacks of these systems exemplify the challenges involved in developing flexible systems that are compatible and can be integrated with embryo culture. Therefore, a continuous advancement in this field need to be done to develop methods for measuring metabolism at a single cell level with a robust, easy to use, reproducible and cost-effective systems.

The focus of the research presented here has been to develop a microfabricated multisensors system for real-time measurement of multiple metabolites in addition to the change in $\mathrm{pH}$ level during cell metabolism. The results of this work demonstrate the capability of a multi-sensors platform to measure the glycolysis and mitochondrial respiration and ectracellular acidification in real-time of single cells during metabolism. The final results have shown the ability of the sensor to monitor a shift from OXPHOS to glycolysis in real-time at single cell level and the correlation between the glycolysis and extracellular acidification. Although there are some commercially available analyzers that are used to measure cell metabolism such as Seahorse XF analyzer [4445], these analyzers are extremely expensive, consumes a lot of media for each test, and requires thousands of cells per each test, and it lacks the real time monitoring of single cells. 
Our design platform can support the ART by providing a simple, flexible, easy to use, inexpensive way with simple pipette system and less labor intensive for measuring multiple analytes at the same time at single cell level. This way of measuring bovine or equine embryos allows tracking the embryo development in the same chamber and the change of metabolic activity during development in a small volume of medium. The micro-chamber design is an important part of the platform that provides sufficient changes of the target analytes in the microenvironment that enables the sensors to measure tiny changes of the target analytes due to cell respiration in a small volume of medium. This setup helps to measure the analytes with a change in concentration ranges from $(0.001$ to 30$) \mathrm{fmol} / \mathrm{s}$ with high specificity which is comparable with what was published in literature. Moreover, our platform allow monitoring the switch in metabolism to glycolysis induced by adding oligomycin as an inhibitor for ATP-synthase. Our platform allow measuring the maximal non-coupled respiratory capacity revealed by carbonyl cyanide-4(trifluoromethoxy)phenylhydrazone (FCCP) titration and it is effect on $\mathrm{pH}$, glucose consumption and lactate production. Other important applications of our platform is evaluating effects of metabolic therapies on oocyte bioenergetics and monitoring mitochondrial function throughout oocyte maturation andblastocyst development to predict embryo viability to compliment assisted reproductive technologies. This platform has a wide potential utility for analyzing small biological samples such as single cells and tumor biopsies for immunology and cancer research applications. However, Limitations of the current setup include challenges associated with handling and positioning of single embryos for analyses, which can be overcome by the addition of microscopy and microfluidic channels for sample visualization and manipulation. Some other limitations include the minor toxicity of the enzyme materials on cells in long term development. Moreover, to monitor the cell in a good environment as close as possible to the body environment, strict control of temperature during monitoring is needed. Finally, other limitations include the lack of periodic calibration process to ensure accuracy. 


\section{Chapter 2: Background and Existing Research Work}

\subsection{Background in Electrochemistry}

Electrochemistry includes techniques that determine an analyte's concentration or characterize an analyte's chemical reactivity by measuring of potential, charge, or current. Electrochemistry is originated from the study of the movement of electrons in an oxidationreduction reaction [46]. The most commonly used electrochemical techniques include amperometry, potentiometry and voltammetry. Amperometry and voltammetry are considered Dynamic techniques in which a current is meausred as a function of a fixed or variable potential [46]. Potentiometry is one of the most important quantitative electrochemical methods in which a potential of an electrochemical cell is measured under static conditions [46].

Electrochemistry provides the ability to do rapid and continuous measurements and observation of real-time changes in diverse biological systems, from single cells [47] to patients in a clinical setting [48]. Additionally, electrochemical sensors can enable label-free, real-time intracellular and extracellular measurements without perturbing the system under investigation [47]. The sensor resolution can be improved by co-locating the sensors with the cells to prevent mixing due to diffusion. Placing both sensors and cells within a micro- environment decreases the distance from cells to sensors [49-51] and increases sensitivity by lowering sample volumes, minimizing the dilution of cellular metabolic products such as lactate into a large extracellular volume, and maximizes the concentration changes with cellular consumption and production, e.g., glucose, oxygen, lactate and acid [50-52, 52-53]. Examples of electrochemical sensors that are widely used for biosensing applications include potentiometric $\mathrm{pH}$ sensors [47-48], Clark oxygen sensors [49-50], and enzymatic based sensors such as glucose and lactate sensors [9-10, 12, 43, 51-53]. There have been several studies that used electrochemical methods for measuring $\mathrm{DO}[50,54]$ glucose $[51,55-56]$, lactate $[51,57]$ and $\mathrm{pH}[58-61]$. 


\subsubsection{Clark Oxygen Electrocehmical Sensor}

Electrochemical sensor relies on the transfer of electrons between an electrode and a chemical redox reaction. Clark sensor is one of the first amperometric sensors that was developed in 1956 by L. C. Clark to measure DO in blood [54]. Clark oxygen sensor measures DO concentration using amperometry [49-50]. As shown in Fig. 2.1, the DO is brought to the surface of WE and a negative voltage is applied between the WE and CE. Electrochemical reduction happens at the WE, and the reduction current of oxygen is proportional to the DO of the sample solution. The reduction at the WE is represented by the following equation [53-54]:

$$
\begin{aligned}
& \mathrm{O}_{2}+2 \mathrm{H}^{+}+2 e^{-} \rightarrow \mathrm{H}_{2} \mathrm{O}_{2} \\
& \mathrm{H}_{2} \mathrm{O}_{2}+2 \mathrm{H}^{+}+2 e^{-} \rightarrow 2 \mathrm{H}_{2} \mathrm{O}
\end{aligned}
$$

The resultant passes through the electrolyte to the CE and oxidizes as following [56]:

$$
2 \mathrm{H}_{2} \mathrm{O} \rightarrow \mathrm{O}_{2}+4 \mathrm{H}^{+}+4 e^{-}
$$

Also, the oxygen reduction current related to the oxygen partial concentration is related by the following equation [56-57]:

$$
I(t)=n F A P_{m}\left(\frac{\partial C}{\partial x}\right), @ x=0
$$

Where $\mathrm{n}$ is the number of electrons, $\mathrm{F}$ is Faraday's constant, $\mathrm{A}$ is surface area of the working electrode, and $\mathrm{P}_{\mathrm{m}}$ is permeability of the membrane.

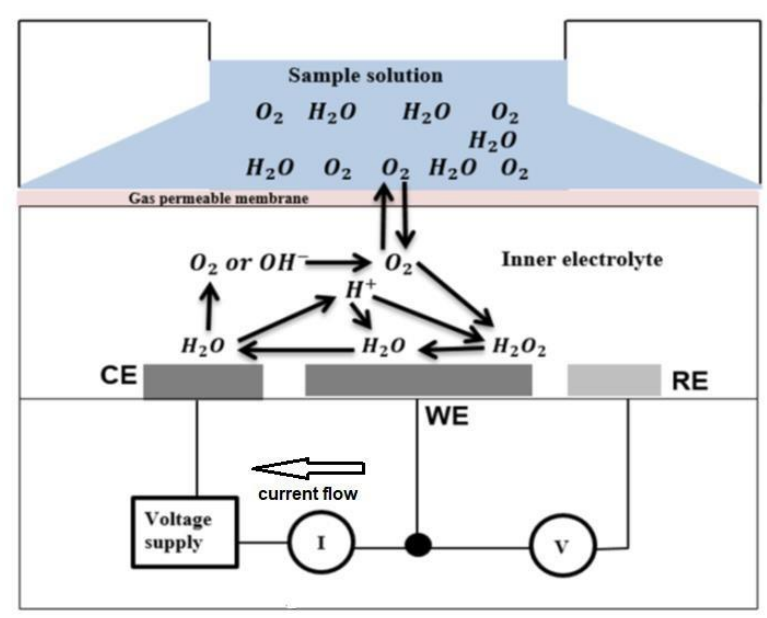

Fig. 2.1. Principle of Clark sensor operation 


\subsubsection{Potentiometric pH Sensors}

In potentiometry the potential of an electrochemical cell is measured under static conditions. The quantitative potentiometric applications use Nernst equation to relate an electrochemical cell's potential to the concentration of electroactive species in the cell [46]. Potentiometric biosensors are based on ion-selective electrodes (ISE) and ion-sensitive field effect transistors (ISFET). The primary outputting signal is possibly due to ions accumulated at the ion-selective membrane interface. Current flowing through the electrode is ideally zero [46].

$\mathrm{pH}$ is defined as the negative logarithm of the hydrogen ion concentration. It represents the relative activity of hydrogen ions in solution. The defintion of $\mathrm{pH}$ was introduced in 1909 by the Danish biochemist, Soren Peter Lauritz Sorensen, and is expressed mathematically as:

$\mathrm{pH}=-\log \left[H^{+}\right]$

$\mathrm{pH}$ measurement is based on the use of a $\mathrm{pH}$ sensitive electrode and a reference electrode. Silicon dioxide $\left(\mathrm{SiO}_{2}\right)$ was commonly used as a $\mathrm{pH}$-sensitive dielectric for the ISFET [59]. For higher $\mathrm{pH}$ responses, $\mathrm{Al}_{2} \mathrm{O}_{3}, \mathrm{Si}_{3} \mathrm{~N}_{4}, \mathrm{Ta}_{2} \mathrm{O}_{5}$, and $\mathrm{SnO}_{2}$ have been used as $\mathrm{pH}$-sensitive dielectrics [59-64]. Indium tin oxide (ITO) has been used as a pH-sensitive electrode because of it's high electrical conductivity, high optical transmittance in the visible region and it is considered anovel $\mathrm{pH}$ sensitive material [65-66].

$\mathrm{pH}$ measurement is potentiometric, where a change in potential between a $\mathrm{pH}$ sensitive electrode and reference electrode is measured in response to change in hydrogen ions concentration without polarizing the electrochemical cell [47-48].

The difference in the potentials of the $\mathrm{pH}$ and reference electrodes provides a millivolt signal proportional to $\mathrm{pH}$. The general mathematical description of $\mathrm{pH}$ Measurement obeys Nernst equation [66]:

$\mathrm{E}=\mathrm{E}_{0}-\frac{R T}{F} \ln \alpha_{\mathrm{H}}$ 
where $\mathrm{F}$ is the Faraday constant, $\mathrm{R}$ is the gas constant, $\mathrm{T}$ is the absolute temperature, $\mathrm{E}_{0}$ is the potential of the reference electrode, and $\alpha_{H}$ is the active ion ratio of hydrogen to hydroxide.

\subsubsection{Enzyme-Based Glucose and Lactate Sensors}

Enzyme-based sensors need enzymes to catalyze a specific biochemical reaction and be stable under the normal operating conditions of the biosensor. They measure the concentration of analytes and/or product of enzymatic reactions that diffuse to the transeducer surface and generate electrical response as shown in Fig. 2.2.

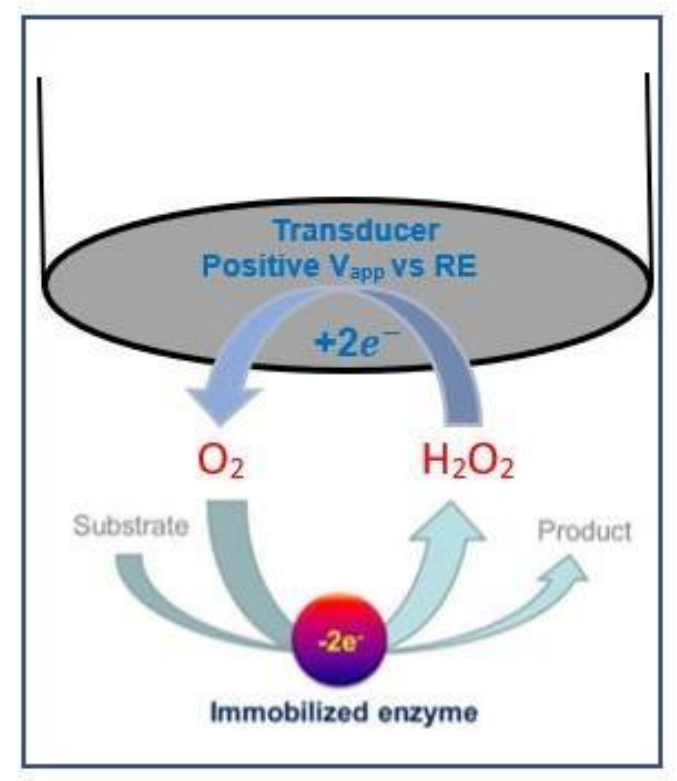

Fig. 2.2. Schematic representation of enzyme based sensors [67].

Glucose and lactate are usually sensed ampeometrically. Their concentrations are measured indirectly by measuring the current generated from the oxidation of hydrogen peroxide generated from the catalysis of glucose or lactate at their working electrodes with their catalysis enzymes $[9-10,51-52]$.

The principle of operation of lactate sensor is described by the following reactions [9, 12]:

$\mathrm{L}-$ lactate $+\mathrm{O}_{2} \stackrel{\text { LOD }}{\longrightarrow}$ Pyruvate $+\mathrm{H}_{2} \mathrm{O}_{2}$

$\mathrm{H}_{2} \mathrm{O}_{2} \rightarrow \mathrm{O}_{2}+2 \mathrm{H}^{+}+2 e^{-}$ 
where lactate oxidase (LOx) catalyzes the oxidation of lactate acid (LA) to pyruvate with the production of hydrogen peroxide $\left(\mathrm{H}_{2} \mathrm{O}_{2}\right)$ in the presence of molecular oxygen. This $\mathrm{H}_{2} \mathrm{O}_{2}$ is then detected on the working electrode as a measurable current, the magnitude of which is directly related to the LA concentration.

Glucose biosensors are based on the glucose oxidase (GOx) enzyme, which catalyzes the oxidation of glucose to gluconolactone which is hydrolyzed to gluconic acid and hydrogen peroxide. The quantification of glucose can be achieved via electrochemical detection of the enzymatic release of $\mathrm{H}_{2} \mathrm{O}_{2}$ [68-69]:

The principle of operation of glucose sensor is described by the following reactions [9-10, 12]:

Glucose $+\mathrm{O}_{2} \stackrel{\text { GOD }}{\longrightarrow}$ gluconolactone $+\mathrm{H}_{2} \mathrm{O}_{2}$

$\mathrm{H}_{2} \mathrm{O}_{2} \rightarrow \mathrm{O}_{2}+2 \mathrm{H}^{+}+2 e^{-}$

\subsection{Optical Methods}

Optical assays have convenient features that make them frequently used for rapid analysis in clinical setups and in laboratories. Fluorescence analysis is one of the most important optical approaches that is widely used for many applications, providing non-invasive, easily operated, disposable, and low-cost assays [70-71]. Several studies have used the optical techniques to measure $\mathrm{pH}$ [72-73], oxygen [71-74], glucose [75] and lactate [74]. Agilent Extracellular Flux Analyzer XF96 developed by Seahorse Bioscience is commercially available and commonly used for measuring oxygen respiration and extracellular acidification measurements (ECAR) in cell cultures [30, 44-45, 76-78]. The sensors in the XF96 instrument are a kind of polymeric gel-based sensor. Seahorse's XF instrument uses a specific chip design to achieve its goal for highthroughput analysis of extracellular $\mathrm{pH}$ and oxygen measurements in their sophisticated and advanced instrument. This instrument can be used to monitor the OXPHOS and glycolysis by measuring oxygen consumption rate $(\mathrm{OCR})$ and ECAR. However, this can be used for understanding cell metabolism in general, but it is certainly insufficient for differentiating different 
cells in terms their metabolism for therapeutic applications. In addition, other important metabolites such as glucose and lactate cannot be measured directly. Moreover, these analyzers are extremely expensive and are incapable of single-cell level resolution.

\subsection{Multi-Sensing Techniques}

Various approaches have been used to facilitate integration of electrochemical sensors for real-time monitoring of multiple analytes [10, 79-82]. Some methods combine amperometric and potentiometric sensors to measure changes to cellular bioenergetics in real-time [10]. The multianalyte microphysiometer (MAMP) employs amperometric glucose, lactate, and oxygen sensors and a pH-sensitive light-addressable potentiometric sensor (LAPS) to measure real-time changes caused by the metabolism of cells immobilized in a microfluidic chamber [51]. The unique combination of these analytes allows for the monitoring of both aerobic and anaerobic respiration. Some other studies used the integration of optical and electrochemical sensors to measure multiple analytes [71]. A multidimensional approach in which responses are obtained simultaneously from multiple analytes offers a more complete understanding of cellular metabolic pathways and changes in these pathways in response to various chemical and biological stimulations [11]. Therefore, different multi-sensing platforms have been developed to assist cell monitoring and drug effects testing. Various techniques of sensors design, and integration have been used to develop multi-sensors platforms. Microelectronic sensors can be used to control the physico-chemical parameters in a measurement chamber containing cells and detect changes of cellular behaviors in response to an experimental treatment [11]. The semiconductor microsensor fabrication process simplify the process of integrating more than one sensor on the same chip, it allows the combination of for example ion-sensitive field-effect transistors (ISFETs) [13, 83-86] with other semiconductor sensors like temperature, light-sensors or interdigitated electrode structures (IDES) [87]. It also allows the integration of high-quality standard electronics on the sensor chip and offers high cost reduction potential by mass production. The FET-based potentiometric sensors can be used for different measurement tasks. Using additional special 
chemical membranes on the gateinsulator of a basic-FET the realisation of ISFETs for different ions $\left(\mathrm{Ca}^{+2}, \mathrm{Na}^{+}, \mathrm{K}^{+}, \ldots\right)$ or ENFETs (enzyme sensitive FETs) for other metabolites (glucose, lactate, ...) becomes feasible $[13,62,88]$. Fig. 2.3 is a Cell Monitoring System (CMS) developed by [11] that has different types of sensors integrated to measure cellular signals.

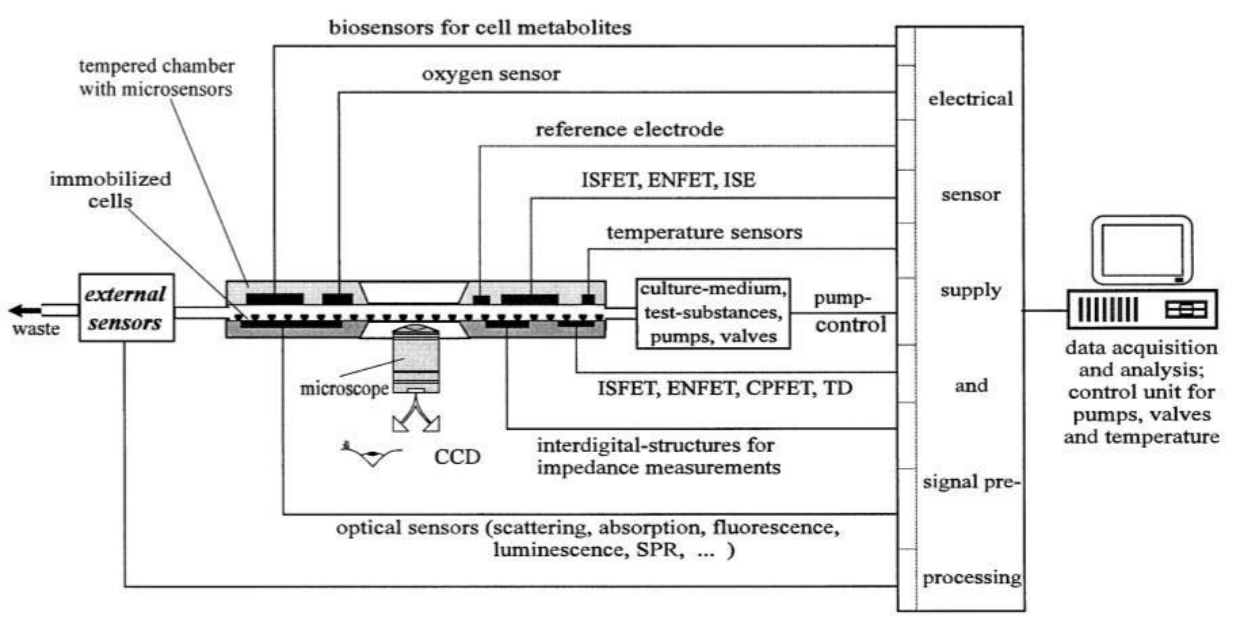

Fig. 2.3. Cell Monitoring System (CMS). It consists of a thermostated cell culture chamber with a fluid handling system and different microsensors. Top sensors are for the detection of chemical parameters in the culture medium and the bottom sensors are directly contacting the cells. (Abbreviations: ISFET, ion selective field-effect transistor; ENFET, enzyme FET; ISE, ion selective electrode; CPFET, cell potential FET; TD, temperature diode; CCD, charge coupled device; SPR, surface plasmon resonance.) [11]

Other studies have developed A microphysiometer by incorporating modified wire electrodes into a standard Cytosensor Microphysiometer plunger to measure changes in extracellular glucose, lactate, oxygen, and acidification rate [10].

Moreover, some other works have used microfabrication techniques to design integrated sensors and built microfludic systems to carry out the timely transport of solutions [12]

\subsection{Existing Research Work}

The techniques that have been successfully used for monitoring cellular activity include electrochemical sensing, scanner electrochemical microscopy, sensing using ion-sensitive field effect transistors, and sensing using solid-state light addressable potentiometric techniques [7- 
15, 31]. Electrochemical sensors have been used successfully in measuring single-cell events using amperometry or voltammetry methods through the development of microelectrodes [7-10].

Many types of solid states and electrochemical sensors have been developed to measure analytes that indicate the metabolic activity in the extracellular environment $[7-10,12,47-53]$. These sensors have been used to determine changes in the analyte concentrations and study the effect of adding chemical or biological agents such as a toxin or drugs [9-12]. Clark sensor is one of the first amperometric sensors that was developed in 1956 by L. C. Clark to measure DO in blood [54]. Clark sensors have been used to measure the DO concentration because of it is simplicity and reliability in measuring DO [49-50]. With the rapid advances in semiconductor technology, various types of miniaturized Clark-type oxygen sensors have been proposed [5152]. The main difficulty of applying the semiconductor manufacturing process for microelectrode fabrication is the incompatibility of the internal electrolyte solution with the fabrication processes [15]. Miyahara et. al. [53] injected the electrolyte manually using a syringe for the charge transfer between electrodes as a postprocessing step to add electrolyte. Niazi et. al. [52] designed microelectrodes with solid electrolyte membrane to improve electrical conductivity and eliminate the need for rehydration. Jobst et. al. [49] used a solid state proton conductive matrix PCM to increase sensor's lifetime and to eliminate the need for rehydration. Clark sensors are also amendable to surface modifications to improve its selectivity using Nafion as solid-state electrolyte as well as membrane [50].

A widely used technique using a light-addressable potentiometric sensor (LAPS) is commercially available for $\mathrm{pH}$ measurement as the Cytosensor Microphysiometer [68-69]. However, $\mathrm{pH}$ sensors suitable for high level of integration are those using ion-sensitive fiel effect transistor (ISFET) as the transducer [ 33-34, 30]. The most commonly used pH-sensitive materials include $\mathrm{SiO}_{2}, \mathrm{Al}_{2} \mathrm{O}_{3}, \mathrm{Si}_{3} \mathrm{~N}_{4}, \mathrm{Ta}_{2} \mathrm{O}_{5}$, Tin oxide $\left(\mathrm{SnO}_{2}\right)$ and indium tin oxide (ITO) because of their higher $\mathrm{pH}$ responses [11-12, 45-50]. ITO is known of its high electrical conductivity and high 
optical transmittance in the visible region and a novel material used as a $\mathrm{pH}$ sensitive electrode [51-52]. Moreover, an extended gate field effect transistor (EGFET) structure has been developed to produce FET isolation from the chemical environment [34]. The main advantages of this structure include light insensitivity, simple to passivate and package, and the shape flexibility in the extended gate area.

Enzymatic sensors have shown their promise for glucose and lactate measurements, and they lend themselves for high level of integration [9-10, 29, 37-38]. The majority of the current glucose and lactate biosensors are of the electrochemical type, because of their better sensitivity, reproducibility, easy to miniaturize, robust, can operate with small sample volumes and easy maintenance as well as their low cost [56-60]. Studies were done to detect extracellular changes in the concentrations of glucose and lactate during cell's metabolism, and different techniques were used to integrate them together or with other sensors such as oxygen and $\mathrm{pH}[9-10,12,55]$. Enzymatic amperometric glucose and lactate biosensors are the most common devices commercially available especially for blood glucose and lactate monitoring, and have been widely studied over the last few decades [56, 57]. However, Amperometric enzyme-based biosensors are subject to interference from chemicals present in the sample matrix, as well as small molecule metabolites, proteins, macromolecules and cells [60].

Other methods of measuring analytes included in metabolism include resonant sensors, conductivity or impedance sensors, and sensors with multiple transduction steps [61-63]. These methods are not aimed at the single-cell level due to size, detection range, or sensitivity incompatibilities [4].

Furthermore, techniques relying on fluorescent labels are dominant in measuring intracellular events [64-66]. However, these techniques have their drawbacks including photobleaching and cytotoxicity that do not exist in the solid state and electrochemical methods [66]. Injecting a cell 
with fluorescent label can also lead to experimental error, since biochemical mechanisms inside of the cell may interact with the label [46-65].

However, most of prior work focused on a single-analyte, whereas simultaneous measurement of multiple analytes can reveal more information about the metabolic pathways of the cell and their response to various chemical and biological stimulations [7-14, 29, 67]. For example, if the extracellular acidification diminishes that does not mean the cell will stop respiration, so monitoring the extracellular acidification alone would lead to the false conclusion that the cells are killed $[12,49]$. To solve this problem mutli-analytes detection is required to give complete information about the cell transport of solutions and conducts electro analysis. Brischwein et al. [8] designed a multi-parametric silicon sensor chips to measure extracellular acidification rate, cellular respiration and cell morphological change of adenocarcinoma colorectal cell. Their design successfully measured all parameters and the effect of different drugs during cell metabolism. However, the $\mathrm{pH}$ sensor has a variable sensitivity of $40-50 \mathrm{mV} / \mathrm{pH}$ lower than the values (55-59) $\mathrm{mV} / \mathrm{pH}$ published in most of studies of $\mathrm{pH}$ sensor [33-34]. The use of ISFET for measuring $\mathrm{pH}$ reduce the sensitivity and stability, induce some leakage and increase noise level. Moreover, oxygen sensor has a very low sensitivity of $0.05 \mathrm{nA} / \mu \mathrm{M}$. The system measures the average from cell populations growing on the chip surface not for single-cell level. And it is not directed to the determination of absolute metabolic rates or distinct cell morphological conditions, but to relative changes of these parameters in the course of an experiment.

Eklund et al. [10] designed a microphysiometer for simultaneous measurement of $\mathrm{pH}$, glucose, lactate and oxygen changes. The design was successfully used to measure Chinese hamster ovary cells and mouse fibroblast and study the effect of different drugs on cell's metabolism. However, the design has some limitation; the use of wire electrodes makes it hard to integrate the design with cell culture, less stability of the sensors due to using two electrodes system with all sensors sharing same reference electrodes, some interference problems happened between sensors, and response time of $\sim 5 \mathrm{~min}$ is very slow. 
Several studies have used Fluorometric enzymatic assays to measure change in cell metabolites including glucose, pyruvate, and lactate. But these methods incredibly labor intensive and pipet construction is complex [25, 26]. Microfabricated approaches have been developed in recent years for studying cellular metabolism, but many of them may not be suitable for integration with culture systems or routine clinical use. One example is the use of electrochemical microphysiometers for monitoring changes in glucose and lactate concentrations in cell cultures using both continuous [27] and discrete [8] fluid flow approaches that needs larger sample volumes and might need difficult calibration. However, the existing methods lack the sophistication and the breath needed to better understand the fundamental mechanisms associated with cell metabolism. One of the important drawbacks of the existing approach is the lack of attention and discussions on electrochemical interference for sensors residing in close proximity within the sensor chamber. 


\section{Chapter 3: Characterization of an $\mathrm{O}_{2}$ Sensor Using Microelectrodes}

\subsection{Background}

To validate the use of Clark principle in measuring DO concentration under a variety of operating conditions, three silicon-based Platinum Pt microelectrodes were used [50]. The main goal of testing different operating conditions was to determine suitable configuration for future integrated oxygen sensor and the suitability for integration with other types of electrochemical sensors on the same substrate surface. The operating conditions tested included the use and the absence of oxygen permeable membrane, the use and absence of electrolyte solution including Nafion as a solid-state electrolyte. The sensor characteristics were evaluated by measuring the change of output current as a function of DO concentrations ranging from $0 \%$ to $100 \%$. The details of this chapter are largely based on a published conference paper, "Characterization of an $\mathrm{O}_{2}$ Sensor Using Microelectrodes [50]".

\section{2 $\mathrm{O}_{2}$ Sensor Design and Characterization}

\subsubsection{Sensor Structure}

The microelectrodes array of twenty-one sensors with different sizes and geometry and arrangement of working electrode (WE), reference electrodes (RE), and counter electrode (CE) designed in [87] was used. The microelectrodes were fabricated using a commercial CMOS process with Pt surface using a lift-off process. The overall chip area is $9 \mathrm{~mm} \times 9 \mathrm{~mm}$ with bonding pads on the exterior that were $160 \mu \mathrm{m} \times 160 \mu \mathrm{m}$ each [87]. For all experiments sensor 17 was used, it has four pairs of WEs with area of $15 \mu \mathrm{m}^{2}$ each and the CE and RE each has an area of $186 \mu \mathrm{m}^{2}$. Fig. 3.1A shows the microphotographs of the microelectrodes used in the experiments. The electrodes were connected to a potentiostat (eDAC, Colorado Springs, CO) through a set of micromanipulator probes as shown in Fig.3.1B. 

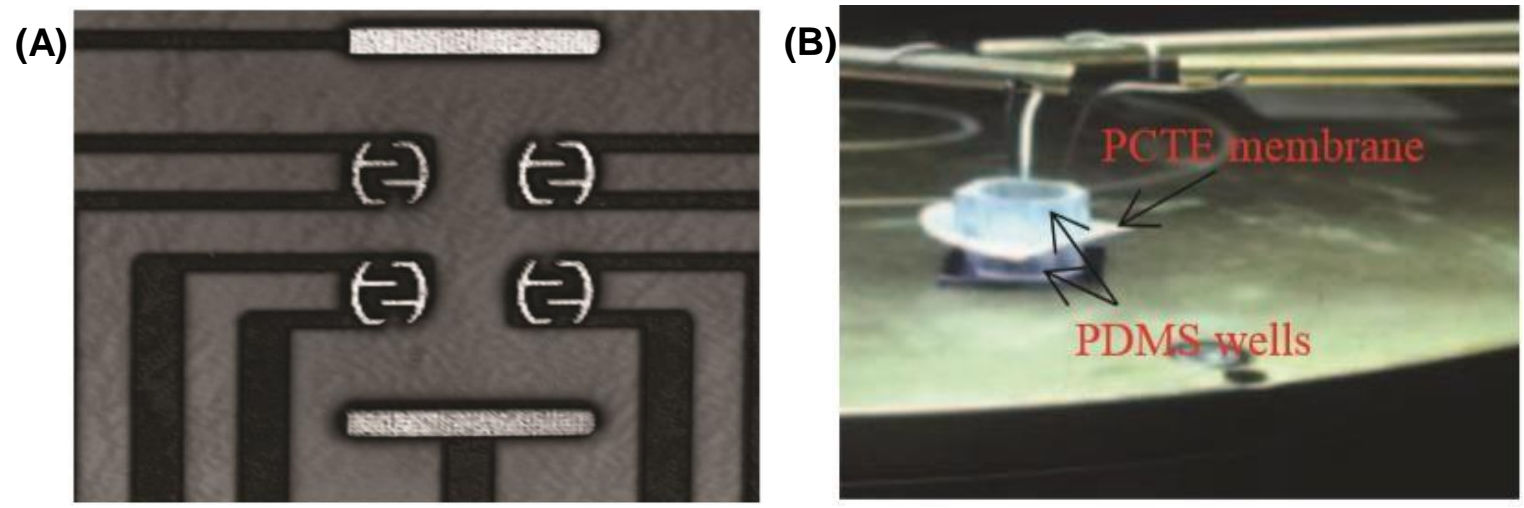

Fig. 3.1. (A) Microphotographs of the microelectrodes used. (B) Close-up view of experiment setup with membrane and PDMS well.

\subsubsection{Materials}

Sodium sulfite was purchased from Eisen-Golden laboratories (Berkeley, California). $0.1 \mathrm{M}$ KCL electrolyte was purchased from HACH Company (Loveland, Colorado). Nafion perfluorinated resin was purchased from Sigma Aldrich (St. Louis, Missouri). A PCTE (Polycarbonate Track Etch Membrane) oxygen-permeable membrane was purchased from Sterlitech Corporation (Kent, Washington).

\subsubsection{Sensor Surface Modification and Operating Conditions}

Experiments were carried out with four different operating conditions: 1) No electrolyte and no membrane; 2) with $0.1 \mathrm{M} \mathrm{KCL}$ electrolyte and PCTE membrane; 3) with Nafion as solid electrolyte and PCTE membrane; 4) with Nafion as solid electrolyte, but no PCTE membrane. During experiments under each condition $\mathrm{Na}_{2} \mathrm{SO}_{3}$ was used as zero oxygen concentration and deionized (DI) water as saturated oxygen concentration. Solutions of different oxygen concentrations were made by mixing $\mathrm{Na}_{2} \mathrm{SO}_{3}$ with $\mathrm{DI}$ water to achieve the desired concentration. All $\mathrm{O}_{2}$ concentrations for measurement by the sensor were validated using the Oakton DO6+ DO meter. The oxygen sensor responses are plotted against the concentrations to determine sensor's sensitivity, linearity, and dynamic range. 


\subsubsection{Optimal Activation Voltage}

A range of voltages from $-0.4 \mathrm{~V}$ to $-0.8 \mathrm{~V}$ was studied to determine the best tradeoff point for activation voltage and the reduction current. Fig. $\mathbf{3 . 2}$ shows the relationship between the activation voltage applied at the WE vs. RE and $\mathrm{O}_{2}$ reduction current. A voltage of $-0.6 \mathrm{~V}$ was chosen for all experiments.

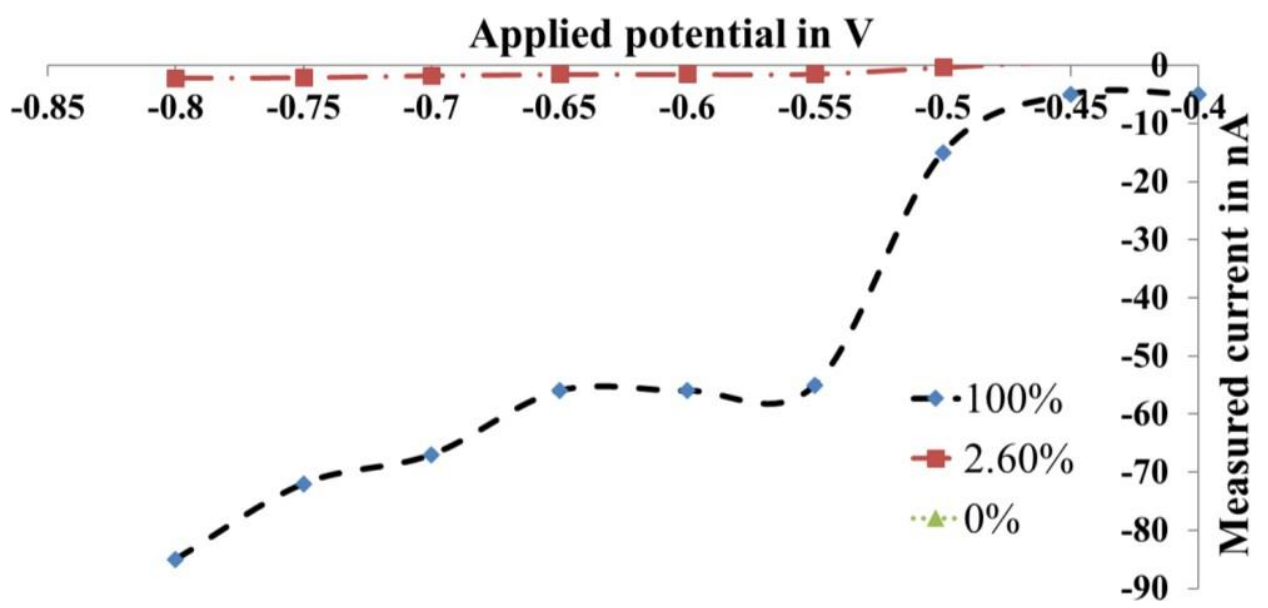

Fig. 3.2. Amperometry measurements at a WE vs. RE voltage range between $0.4 \mathrm{~V}$ and $-0.8 \mathrm{~V}$.

\subsubsection{Calibration Results}

The calibration curves for the Clark sensor under four different conditions are shown in Figs. 3.3 and 3.4. For all conditions, the sensor output current increases with increase in $\mathrm{O}_{2}$ concentration in a linear relationship with the correlation coefficients between 0.88 and 0.98 , limit of detection (LOD) of $1.7 \%$, and sensitivity range of $(0.387$ to 1.27$) \mathrm{nA} / \%$. The factors that can contribute to variations of the measurement results include variations in pipetting speed and direction, electrochemical crosstalk between electrodes, and atmospheric oxygen diffusing through PDMS (Polydimethylsiloxane) well. Variations can be larger under the condition without electrolyte and membrane because the pipetted solution came in direct contact with the electrode surface causing disturbance in the immediate area of the electrode surface. The results have shown that using Nafion as solid-state electrolyte and oxygen-permeable membrane provides the 
best linearity and stability, and good sensitivity. However, using Nafion as solid-state electrolyte as well as membrane also shows comparable linearity and sensitivity. The use of Nafion only is more compatible with integration and multiplexed sensing applications using other sensing modals.
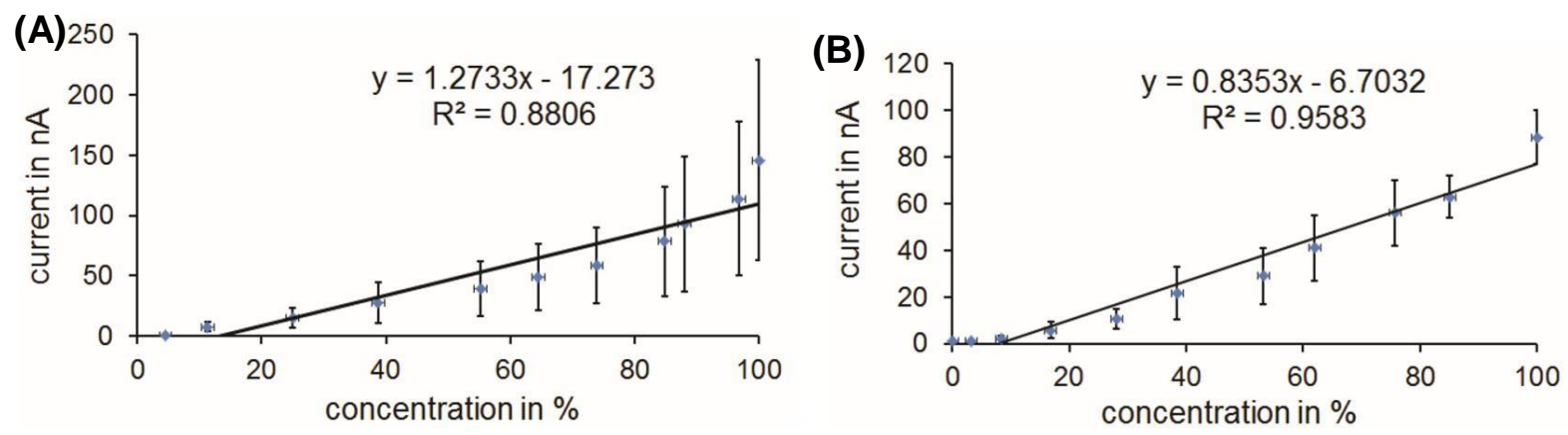

Fig. 3.3. Calibration curves: (A) without electrolyte and membrane. (B) with $0.1 \mathrm{M} \mathrm{KCL}$ electrolyte and PCTE membrane. The error bars represent the standard deviation (SD) between four data points for the same condition.
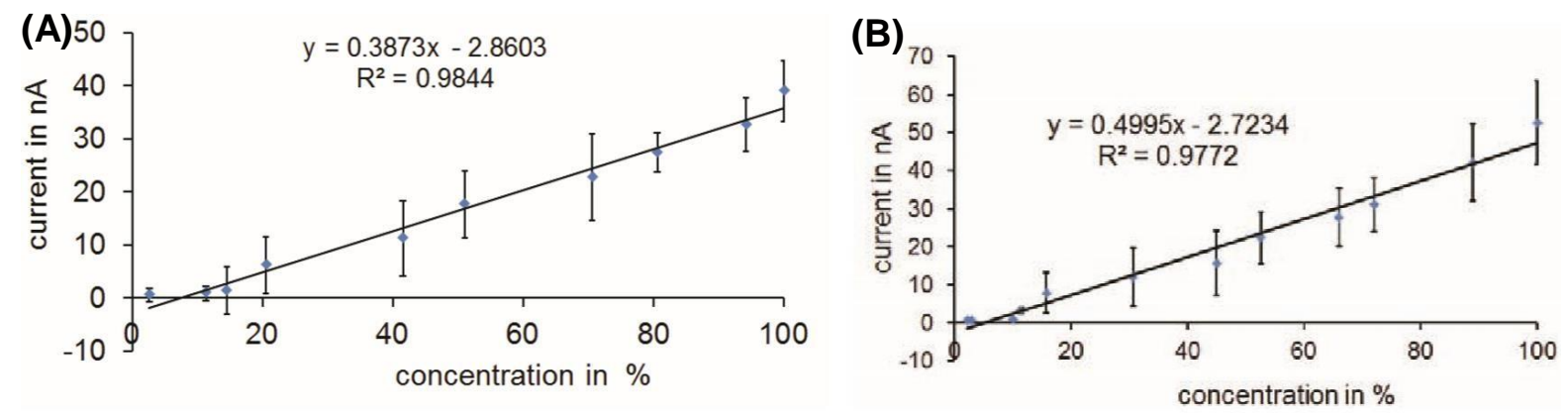

Fig. 3.4. Calibration curves: (A) with Nafion and PCTE membrane. (B) with Nafion but without PCTE membrane. The error bars represent the SD between four data points for the same condition. 


\section{Chapter 4: Monitoring Oocyte/Embryo $\mathrm{O}_{2}$ Respiration Using Three Electrodes Clark}

Sensor

\subsection{Background of The Research}

After validating the use of Clark principle in measuring DO in Chapter 3, A disposable threeelectrode, Clark-type biosensor suitable for mitochondrial respirometry in single oocytes and embryos was developed. The biosensor described in this chapter was embedded in a PMMA (polymethyl methacrylate) micro-chamber to allow investigation of single oocytes/embryos immersed in up to $100 \mu \mathrm{L}$ of respiration buffer. The microchamber was sealed to avoid oxygen exchange between the inside of the chamber and the atmosphere, while being maintained at a temperature of $38.5^{\circ} \mathrm{C}$ to preserve cell viability. The measurements from the sensor system revealed basal cell respiration supported by endogenous substrates, respiration associated with proton leak induced by inhibition of the adenosine triphosphate (ATP) synthase (complex V) with oligomycin, and the maximal noncoupled respiratory capacity revealed by carbonyl cyanide-4(trifluoromethoxy)phenylhydrazone (FCCP) titration. The details of this chapter are largely based on a published journal paper, "Monitoring oocyte/embryo respiration using electrochemical-based oxygen sensors [89]".

\subsection{Sensor Structure}

\subsubsection{Sensor Electrodes}

All experiments used the screen-printed electrodes DS550 (Dropsens, Llanera, Spain). The electrodes were printed on ceramic substrate of $33 \mathrm{~mm}$ length, $10 \mathrm{~mm}$ width, and $0.5 \mathrm{~mm}$ height. The electrochemical cell consists of: circular Platinum (Pt) working electrode (WE) of $4 \mathrm{~mm}$ diameter, curved Pt counter electrode (CE), and small curved silver (Ag) pseudo reference electrode (RE), and silver electric contact as shown in Fig. 4.1. 


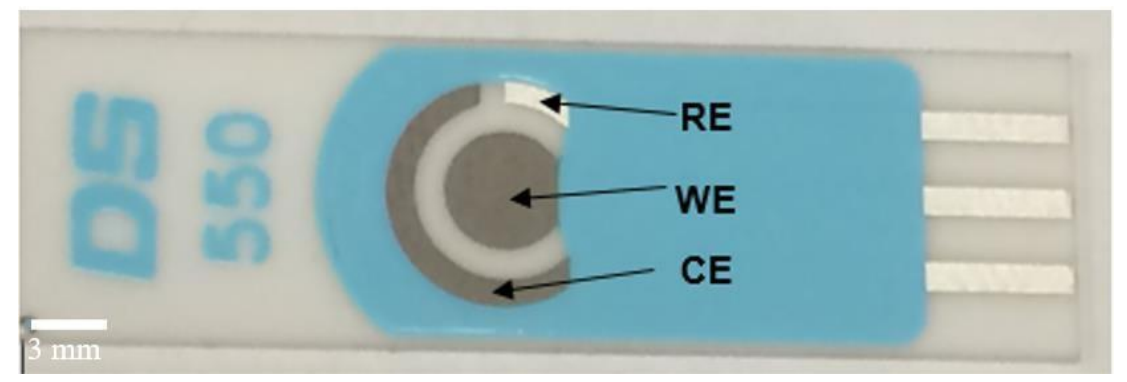

Fig. 4.1. DS550 screen printed electrodes.

\subsubsection{Microchamber Design}

A micro-chamber for housing single oocyte and embryo cells was laser cut from a $4.76 \mathrm{~mm}$ thick PMMA sheet with a $1.59 \mathrm{~mm}$ thick PMMA slit to allow the electrode chip to be mounted. The screen-printed sensor chip was placed on a flat PMMA base, overlaid on top by the cone shaped micro-chamber. The assembly between the base and the cone-shaped micro-chamber is sealed by a rubber ring. The cone-shaped chamber was manually press drilled using a $1 / 2 " 82$-degree countersink drill bit (Vermont American), resulting in a base diameter of $8 \mathrm{~mm}$, top chamber inlet diameter of $2 \mathrm{~mm}$, and $3.5 \mathrm{~mm}$ height truncated cone as shown in Fig. 4.2. The setup holds up to $100 \mu \mathrm{L}$ of buffer with a $2 \mathrm{~mm}$ diameter top opening to allow loading of buffer and oocyte/embryo cells into the chamber.

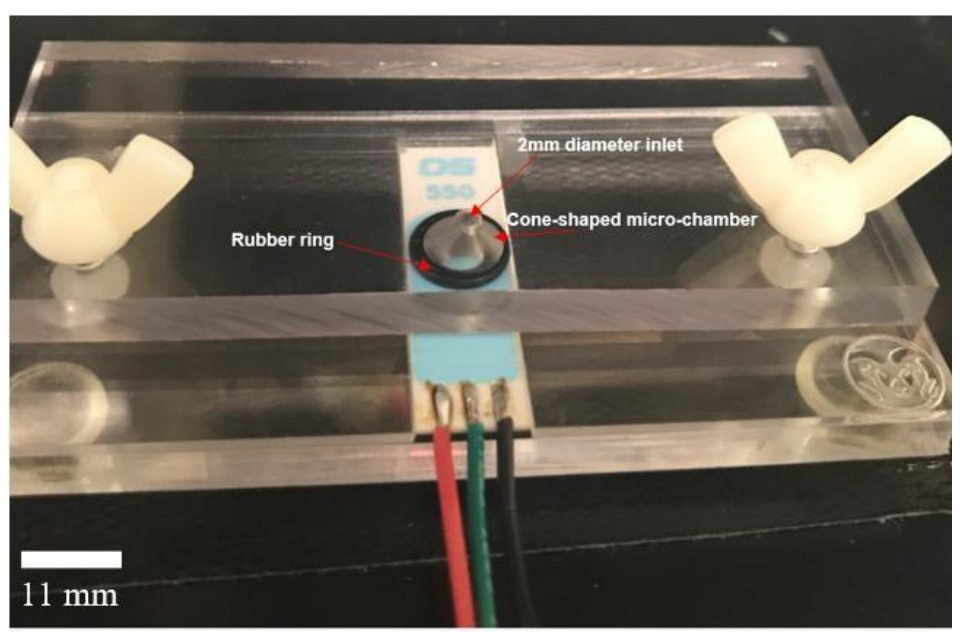

Fig. 4.2. Single channel sample reservoir of 100uL volume max. 


\subsubsection{Sensor Surface Modification}

Among different surface preparation methods, Nafion was chosen as the solid-state electrolyte as well as the membrane. Using Nafion only is more compatible with integration and multiplexed sensing applications than using other electrolytes and membranes as described in Section 3.2.5. A $2 \mu \mathrm{L}$ of Nafion solution was added and distributed using a pipette to cover the surface of the WE and allowed to dry for 20 minutes to form a thin layer of Nafion on the WE.

\subsection{Sensor Characterization}

\subsubsection{Oxygen Reduction Voltage}

Activation voltages range from $-0.6 \mathrm{~V}$ to $-0.8 \mathrm{~V}$ has been suggested in the literature [90-93]. However, the actual reduction voltage also depends on electrode size and geometry. To determine the required activation voltage for measuring DO using the sensor, cyclic voltammetry (CV) was performed, the potential was swept from $-1.5 \mathrm{~V}$ to $0.5 \mathrm{~V}$ at $100 \mathrm{mV} / \mathrm{s}$ with respect to $\mathrm{Ag}$ pseudo-reference electrode. The CV results are shown in Fig. 4.3.
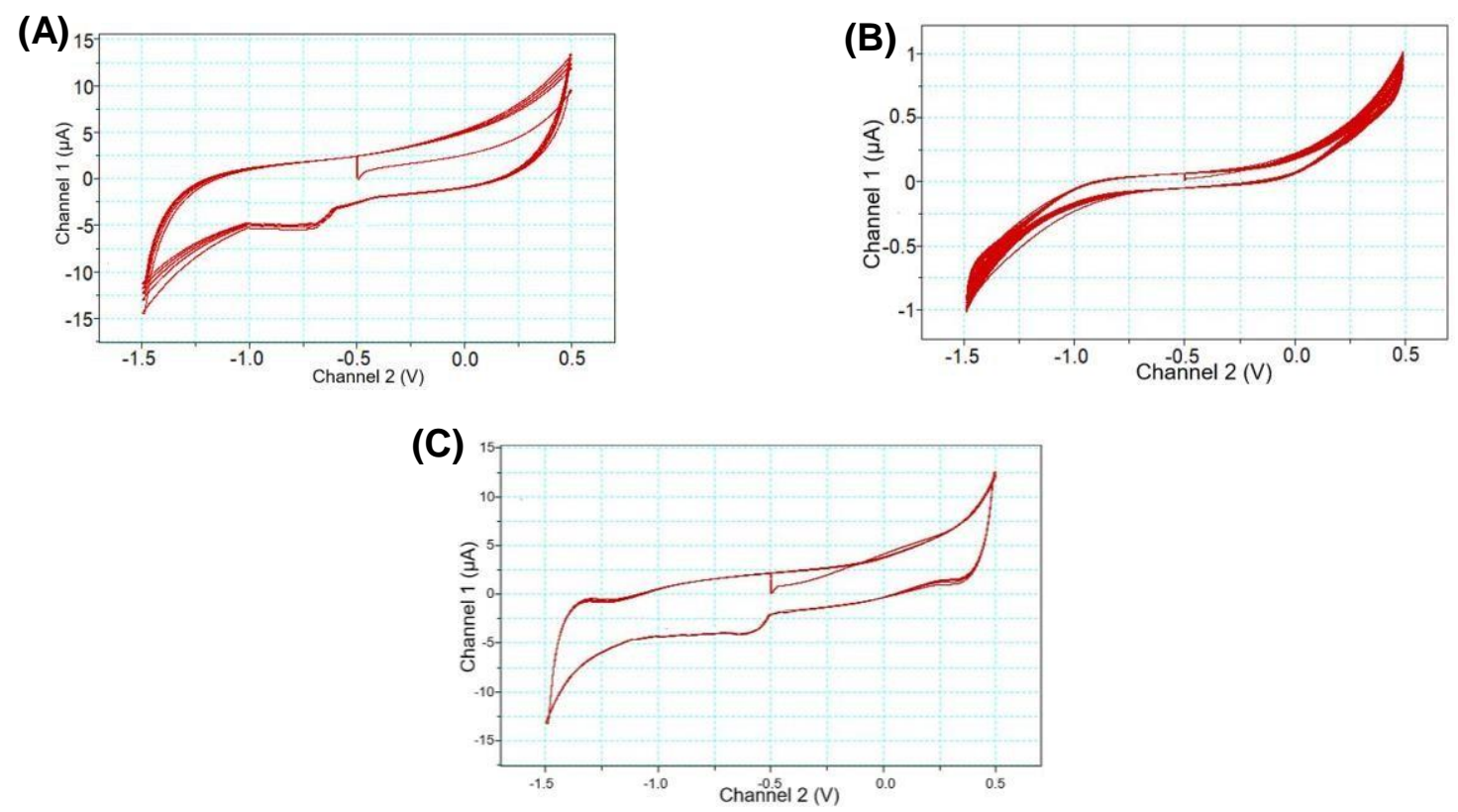

Fig. 4.3. (A) $\mathrm{CV}$ when DI water was tested. (B) $\mathrm{CV}$ when $\mathrm{Na}_{2} \mathrm{SO}_{3}$ was tested. (C) $\mathrm{CV}$ when MiR05 buffer was tested. 
When DI water or MiR05 buffer was tested, a noticeable cathodic peak was observed in the range $-1 \mathrm{~V}$ to $-0.7 \mathrm{~V}$, whereas when sodium sulfite was tested, negligible reduction current and no cathodic peak was observed as shown in Fig. 4.3. To achieve the least interference from other molecules in the solution, low voltage is preferred; in all experiments, a reduction voltage of -0.75 V was used.

\subsubsection{Sensor Calibration}

Taking in consideration the following reaction between oxygen and sodium sulfite:

$2 \mathrm{Na}_{2} \mathrm{SO}_{3}+\mathrm{O}_{2} \rightarrow 2 \mathrm{Na}_{2} \mathrm{SO}_{4}$

The theoretical number of parts of $\mathrm{Na}_{2} \mathrm{SO}_{3}$ required to consume 1 part of $\mathrm{O}_{2}$, can be calculated as:

$\frac{2\left(126_{\mathrm{mo}}^{-g} \mathrm{Na}_{2} \mathrm{SO}_{3}\right)}{32 \frac{\mathrm{g}}{\mathrm{mo} \mathrm{O}} \mathrm{O}}=7.88$

Therefore, about 8 parts of $\mathrm{Na}_{2} \mathrm{SO}_{3}$ are needed to consume each part of oxygen theoretically. Sodium sulfite $\left(\mathrm{Na}_{2} \mathrm{SO}_{3}\right)$ was purchased from Eisen-Golden laboratories (Berkeley, California) and was used for measurements as the zero-oxygen concentration. DI water or MiR05 buffer was used for measurements as the saturated DO concentration. To determine the calibration curve and linearity of DO sensor, DO concentration was changed by adding $0.1 \mathrm{M} \mathrm{Na}_{2} \mathrm{SO}_{3}$ to the saturated solution in steps with continuous stirring to produce different oxygen concentrations for generating the calibration data as explained in [90]. All $\mathrm{O}_{2}$ concentration measurements were made at room temperature of $25^{\circ} \mathrm{C}$ and validated using calibrated Oakton DO6+ DO meter. The DO reduction current was measured 0.5 min after each addition of $\mathrm{Na}_{2} \mathrm{SO}_{3}$. The oxygen sensor responses are plotted against the concentrations (relative percentage of DO) to determine sensor's sensitivity, linearity, and dynamic range. Furthermore, the DO concentration in the MiR05 respiration buffer in $\mu \mathrm{M}$ corresponding to the measured current was calculated at $158 \mu \mathrm{M}$ based on the average barometric pressure of the experiment location (Fort Collins, Colorado (84.8 kPA)) 
at the temperature of $38.5{ }^{\circ} \mathrm{C}$, corrected for the slightly lower oxygen solubility of the MiR05 respiration buffer (0.92) compared to water, as determined by Rasmussen and Rasmussen [91]. Calibration curves of the measured reduction current versus the relative percentage of DO concentration in DI water and in the GMOP respiration buffer were generated.

\subsubsection{Reproducibility Test}

DI water and $0.1 \mathrm{M} \mathrm{Na}_{2} \mathrm{SO}_{3}$ were added in a sequential cycle to demonstrate the repeatability of the sensor. In each step, $0.1 \mathrm{M} \mathrm{Na}_{2} \mathrm{SO}_{3}$ solution with zero oxygen concentration was added using a pipette and reduction current was measured, then the solution was pipetted out after taking each measurement and the electrodes were dried before adding another solution. DI water was added to measure the reduction current corresponding to high oxygen concentration. This process was repeated multiple times to ensure sensor repeatability. The sensor showed good reproducibility with a mean of $3.27 \mu \mathrm{A}$ and a standard deviation of (47 nA or $2.44 \mu \mathrm{M})$ in the saturated DO level $(21 \%$ or $170 \mu \mathrm{M})$ using DI water, and a mean of (27 nA or $1.4 \mu \mathrm{M})$ and a standard deviation of $(6 \mathrm{nA}$ or $0.311 \mu \mathrm{M})$ in the minimum oxygen level $(0.23 \%$ or $1.87 \mu \mathrm{M})$ using $\mathrm{Na}_{2} \mathrm{SO}_{3}$. The results also demonstrated a 0\%-to-21\% oxygen response time of $30 \mathrm{~s}$ as shown in Fig. 4.4A. A similar reproducibility experiment was done using MiR05 buffer as saturated DO concentration instead of DI water, the sensor showed a good reproducibility with a mean of 4.09 $\mu \mathrm{A}$ and a standard deviation of $(74 \mathrm{nA}$ or $3.07 \mu \mathrm{M})$ in the saturated DO level $(21 \%$ or $158 \mu \mathrm{M})$ using MiR05 buffer; and the mean of (30 nA or $1.17 \mu \mathrm{M})$ and a standard deviation of (4.8 $\mathrm{nA}$ or $0.188 \mu \mathrm{M})$ in the minimum oxygen level $(0.23 \%$ or $1.73 \mu \mathrm{M}))$ using $\mathrm{Na}_{2} \mathrm{SO}_{3}$ as shown In Fig. 4.4B. The figures show absolute values of current (current sign is not shown). 

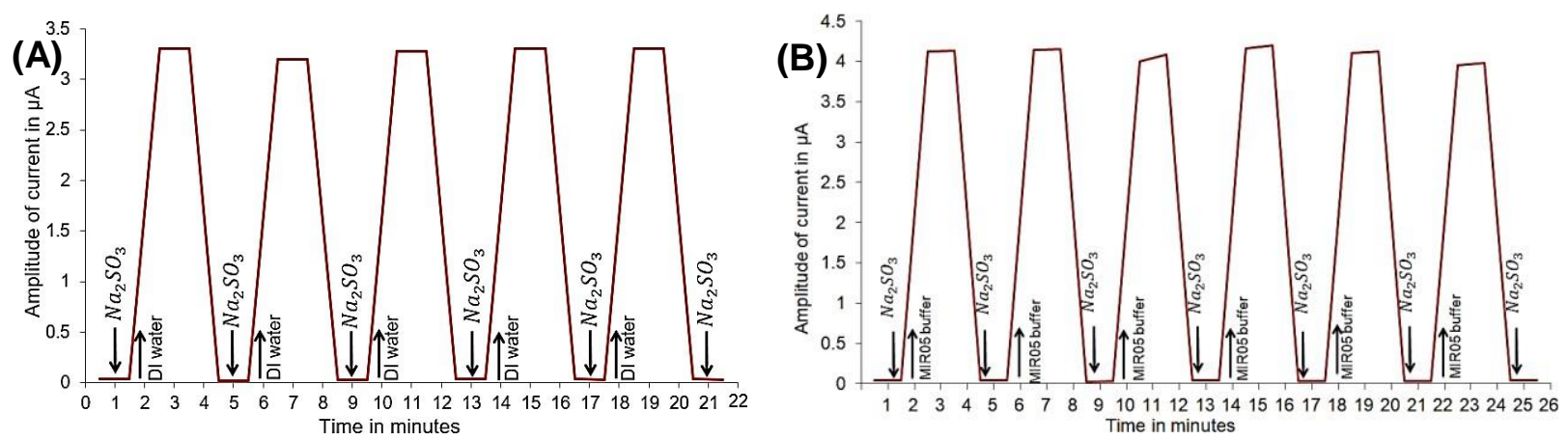

Fig. 4.4. Time response and reproducibility of the Clark sensor when: (A) DI water and $\mathrm{Na}_{2} \mathrm{SO}_{3}$ was used. (B) MiR05 buffer and $\mathrm{Na}_{2} \mathrm{SO}_{3}$ was used.

\subsubsection{Verification of Airtightness of the Micro-Chamber}

To determine whether the micro-chamber is airtight, a $0.1 \mathrm{M} \mathrm{Na}_{2} \mathrm{SO}_{3}$ was loaded into the micro-chamber, and continuous operations of up to 30 minutes were performed to determine whether oxygen exchange between the micro-chamber and the atmosphere occurred. To test the ability of paraffin oil to seal the micro-chamber, paraffin oil was added to the load cap at the top of the micro-chamber, and the change in oxygen level inside the micro-chamber was measured and compared with the setup without paraffin oil sealing. The sensor measurement output without the micro-chamber being sealed shows a change in the measured current across time reflecting a diffusion of atmospheric oxygen into the micro-chamber as shown in Fig. 4.5A. The sensor measurement, when the micro-chamber was sealed by paraffin oil, indicate no oxygen diffusion from the atmosphere as shown in Fig. 4.5 (B).
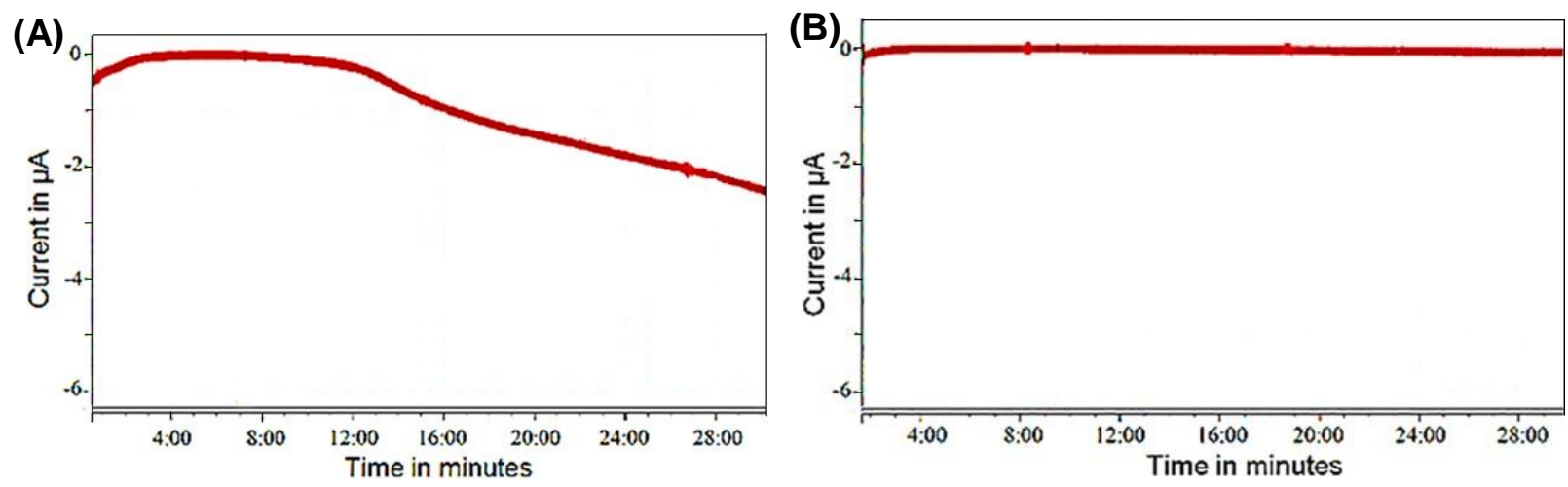

Fig. 4.5. (A) Time response when $0.1 \mathrm{M} \mathrm{Na}_{2} \mathrm{SO}_{3}$ was tested with no-top sealing. (B) Time response when $0.1 \mathrm{M} \mathrm{Na}_{2} \mathrm{SO}_{3}$ was tested with paraffin oil as a top sealing. 


\subsection{Measurement Setup}

\subsubsection{Oocytes and Embryos}

Equine and bovine oocytes were collected from live mares or bovine ovaries from a slaughterhouse. Equine oocytes were matured in Tissue Culture Medium 199 (Gibco ${ }^{\mathrm{TM}}$, Grand Island, NY) with additions of $10 \%$ fetal calf serum, $0.2 \mathrm{mM}$ pyruvate and $25 \mu \mathrm{g} / \mathrm{mL}$ gentamicin, with or without the addition of hormones and growth factors $(15 \mathrm{ng} / \mathrm{mL} \mathrm{FSH}, 1 \mu \mathrm{g} / \mathrm{mL} \mathrm{LH}, 1 \mu \mathrm{g} / \mathrm{mL}$ $\mathrm{E}_{2,}, 200 \mu \mathrm{g} / \mathrm{mL} \mathrm{P}_{4}, 10 \mathrm{ng} / \mathrm{mL}$ IGF and $100 \mathrm{ng} / \mathrm{mL}$ EGF) depending on if maturation inducing drugs had been administered to the donor mare and on morphology of the granulosa and cumulus cells [92]. Cumulus oocytes were classified as mature or not mature and invariant amounts of hormones were only added if the oocytes were considered not mature. Equine oocyte maturation was performed in an atmosphere of $6 \% \mathrm{CO}_{2}$ at $38.2{ }^{\circ} \mathrm{C}$. Bovine oocyte maturation, fertilization and embryo culture were performed as previously described and using CDM1/CD/M2 sequential embryo culture media [93]. Prior to analyses, oocytes or embryos were transported $<20$ min to another laboratory in medium TCM 199 with Hanks' salts, 10\% newborn calf serum, $0.2 \mathrm{mM}$ pyruvate and $25 \mu \mathrm{g} / \mathrm{mL}$ gentamicin) while housed within an insulated container.

\subsubsection{Stimuli for Oocytes and Embryos}

Carbonyl cyanide-4-(trifluoromethoxy) phenylhydrazone (FCCP: 1-4 $\mu \mathrm{M})$ was purchased from Sigma Aldrich (St. Louis, Missouri) and was used as a potent protonophore uncoupler of mitochondrial oxidative phosphorylation. Oligomycin $(1 \mu \mathrm{M})$ was purchased from Sigma Aldrich (St. Louis, Missouri) and was used as ATP synthase inhibitor.

\subsubsection{Experiment Protocol}

Prior the experiments the WE was covered by a Nafion layer as solid-state electrolyte as well as membrane. The temperature of the medium was maintained at $38.5{ }^{\circ} \mathrm{C}$ by placing the device on the slide warmer during the experiments. The applied potential during all the amperometric experiments was set at $-0.75 \mathrm{~V}$. The electrodes were rinsed using DI water and electrochemically cleaned before each test. The pulses used for cleaning were $2 \mathrm{~V}$ peak-to-peak with a sampling 
rate of $50 \mathrm{mV} / \mathrm{s}$ for one minute. MiR05 respiration buffer $(90 \mu \mathrm{L})$ was placed in the micro-chamber and overlaid with $90 \mu \mathrm{L}$ Paraffin oil to seal the micro-chamber and the baseline current was measured where it took 1-2 min for the current to stabilize before injecting the cell. The oocyte/embryo was transferred to the micro-chamber by pipetting through the top oil layer on to the center of the WE under the microscope. When measuring respiration of multiple cells, they were placed on the WE with all of them approximately at same distance from the center of the WE. Oxygen consumption was measured by monitoring the decrease in the oxygen reduction current over time during cell respiration.

\subsubsection{Model Used for Calculations}

The change in the measured reduction current over a given period was converted to its equivalent oxygen consumption within a defined volume in $\mathrm{fmol} / \mathrm{s}$ using the calibration curve of the sensor.

Assuming the oxygen concentration gradient within the micro-chamber towards the cell reached its equilibrium state when measurement was taken (i.e. changes in reduction current over time is constant), the defined volume is the volume immediately surrounding the cell inside the micro-chamber. The radius of the WE is $2 \mathrm{~mm}$. The cell height ranges from 150-200 $\mu \mathrm{m}$ and the cell is placed on the WE during experiments. The defined volume for cell oxygen consumption calculation is the area of the WE time the height of $250 \mu \mathrm{m}$, i.e. $3.14 \mathrm{~mm}^{3}$. The height of $250 \mu \mathrm{m}$ was chosen by considering the maximum cell height (approximately $200 \mu \mathrm{m}$ ) and another 50-100 $\mu \mathrm{m}$ above the cell which is the volume immediately surrounding the cell. The schematic view of the micro-chamber is shown in Fig. 4.6.

Oxygen consumption in $\mathrm{fmol} / \mathrm{s}(\mathrm{OC}(\mathrm{fmol} / \mathrm{s}))$ is defined as:

$$
O C\left(\frac{f m o l}{s}\right)=\frac{\Delta C}{\Delta t} \times V
$$

where $\Delta \mathrm{C} / \Delta \mathrm{t}$ is the change in oxygen concentration over a given time, and $\mathrm{V}$ is the defined volume within the micro-chamber. Equation 3 can be re-written as: 
OC $\left(\frac{f m o l}{s}\right)=\frac{\Delta I / \Delta t}{\Delta I / \Delta C} \times V$

where $\Delta \mathrm{l} / \Delta \mathrm{t}$ is the change of reduction current over a given time obtained during experiments, and $\Delta \mathrm{l} / \Delta \mathrm{C}$ is the calibration curve.

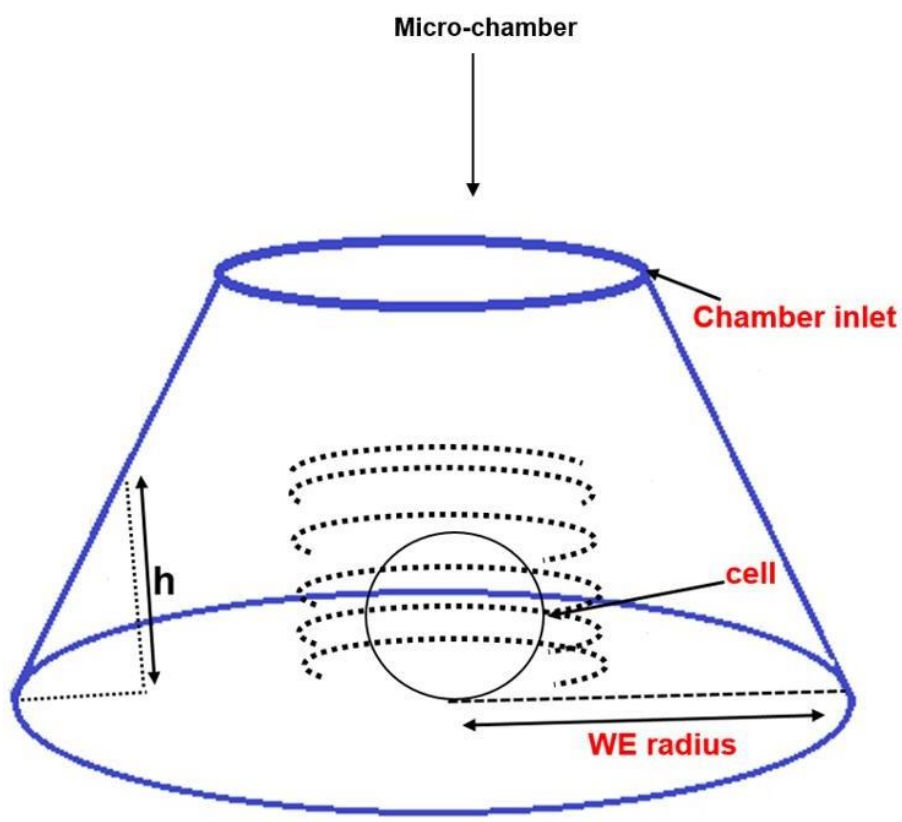

Fig. 4.6 Schematic view of the oxygen consumption measurement for sample located in a coneshaped microwell. (drawing is not to scale)

\subsection{Results and Discussion}

\subsubsection{Calibration Results}

The calibration curves in Figs. 4.7 (A and B) were obtained for the sensor when Nafion was used as a solid-state electrolyte as well as membrane. The sensor can measure a DO range of (0-170) $\mu \mathrm{M}$ with a sensitivity of $0.022 \mu \mathrm{A} / \mu \mathrm{M}$ was found by taking the slope of the calibration curve. The sensor output current increased with the increase in $\mathrm{O}_{2}$ concentration in a linear relationship and the correlation coefficient of 0.9807 when MiR05 bufferwas used and 0.9825 when DI water was used. 

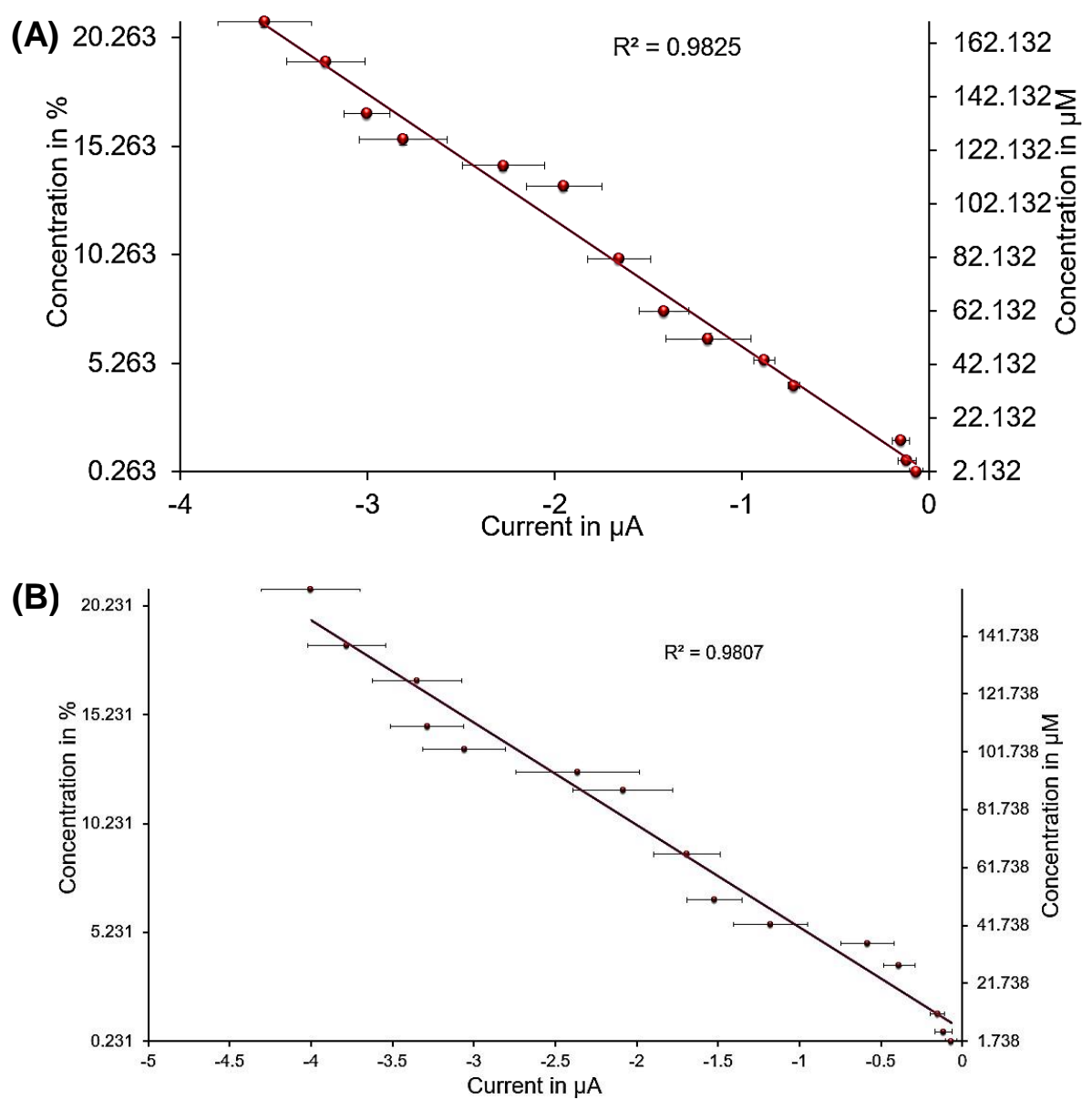

Fig. 4.7. Calibration curve with Nafion used as solid-state electrolyte: (A) when DI water was used. (B) when MiR05 buffer was used. Left y-axis represents concentration in \%, right y-axis is the corresponding concentration in $\mu \mathrm{M}$. The error bars represent SD of ten calibration data points under the same conditions.

\subsubsection{Measurement of Basal Mitochondrial Respiration in Individual Equine Oocytes}

The vast majority of oxygen consumption by intact living cells is associated with oxidative phosphorylation (OXPHOS) by mitochondria, whereby electrons removed from endogenous metabolic intermediates (oxidation) are transferred through a series of inner membrane protein complexes before reducing oxygen to form water at cytochrome oxidase (respiration). This transfer of electrons is linked to proton translocation across the inner mitochondrial membrane, which generates a proton gradient that flows through and "powers" the ATP synthase to phosphorylate ADP to ATP. Thus, cellular respiration is coupled to ATP synthesis through generation of an inner membrane proton gradient. The oxygen consumption rate of individual 
equine oocytes was determined by monitoring the linear decrease in reduction current for $20-30$ minutes following stabilization of the electrode signal in $90 \mu \mathrm{L}$ respiration buffer alone as shown in Fig. 4.8A. The measured respiration rate increases accordingly if additional cells are added to the micro-chamber. A nearly-linear increase in basal respiration rate with the increase in the number of cells in the micro-chamber was achieved as shown in Fig. 4.8B.

(A)
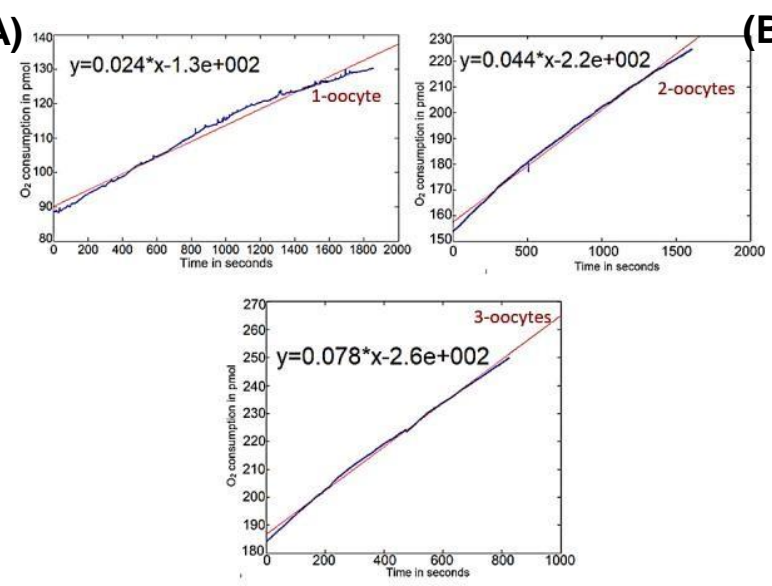

(B)

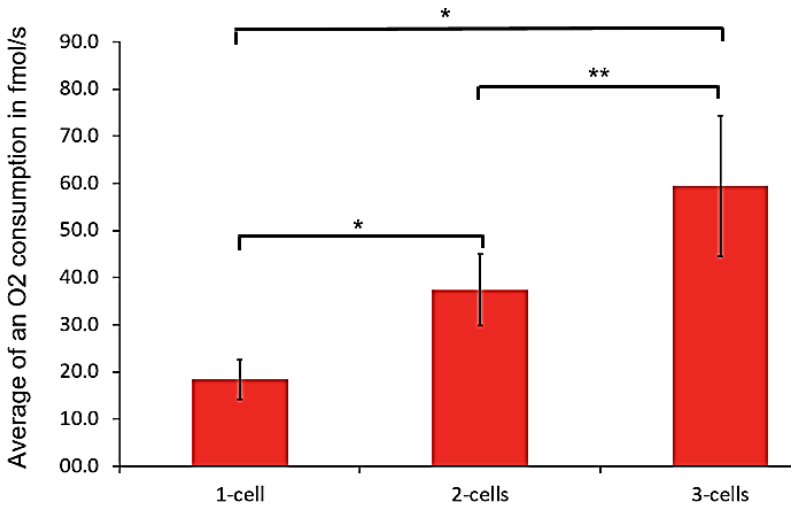

Fig. 4.8. (A) An example of the difference in the slope of the basal respiration of one, two or three bovine oocytes. (B) The average basal respiration of one, two or three bovine oocytes (error bars represent SD between 7 different experiments). ${ }^{*}$ paired t-test: $p<0.01$; ** paired t-test: $p<0.05$.

\subsubsection{Effect of Oligomycin Addition on Cell Respiration of Equine Oocytes}

Blockade of proton flux through the ATP synthase with oligomycin severely limits the rate of cellular respiration by minimizing dissipation of the inner membrane proton gradient [94-95]. To confirm that the rate of oxygen consumption detected was associated with oxidative phosphorylation, $1 \mu \mathrm{M}$ oligomycin was added to the microchamber following stabilization of the basal oocyte respiration signal. The results are shown in Figs. 4.9 and 4.10. As expected, the addition of oligomycin reduced respiration of the cell to about $1 / 7$ of it is basal respiration value, confirming that essentially all the oxygen consumption being monitored was oocyte mitochondrial respiration. This reflects a high degree of oxidative phosphorylation "coupling" in oocytes, indicated by nearly undetectable levels of "leak" respiration in the absence of ATP synthesis. These results agree with what was achieved by Sugimura et al. [95], where $2 \mu \mathrm{g} / \mathrm{mL}$ oligomycin 
was used to inhibit ATP synthase (complex V) in bovine oocytes and a decrease in basal respiration at different stages and ages of the cells was reported in their paper.
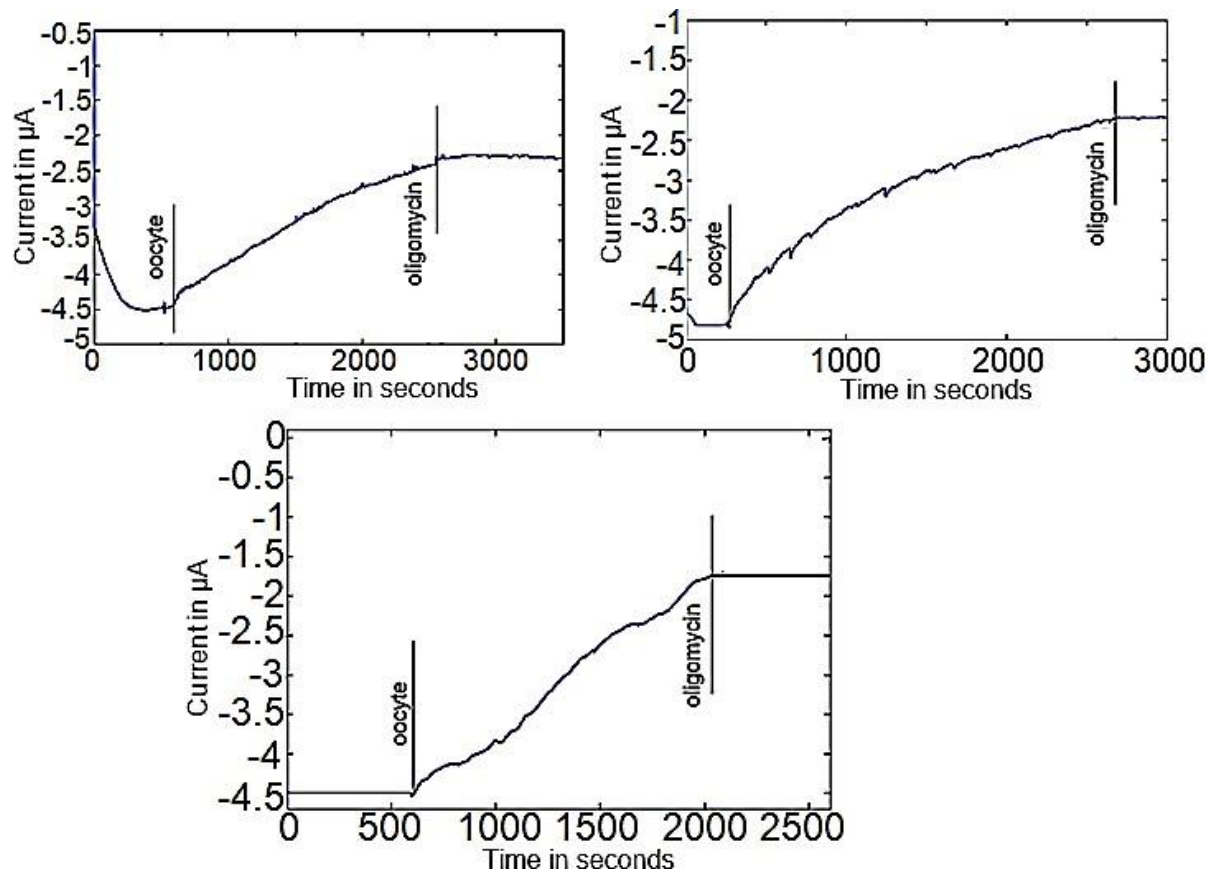

Fig. 4.9. Amperometry results for three equine oocytes: basal respiration after adding the oocyte followed by the effect of oligomycin.

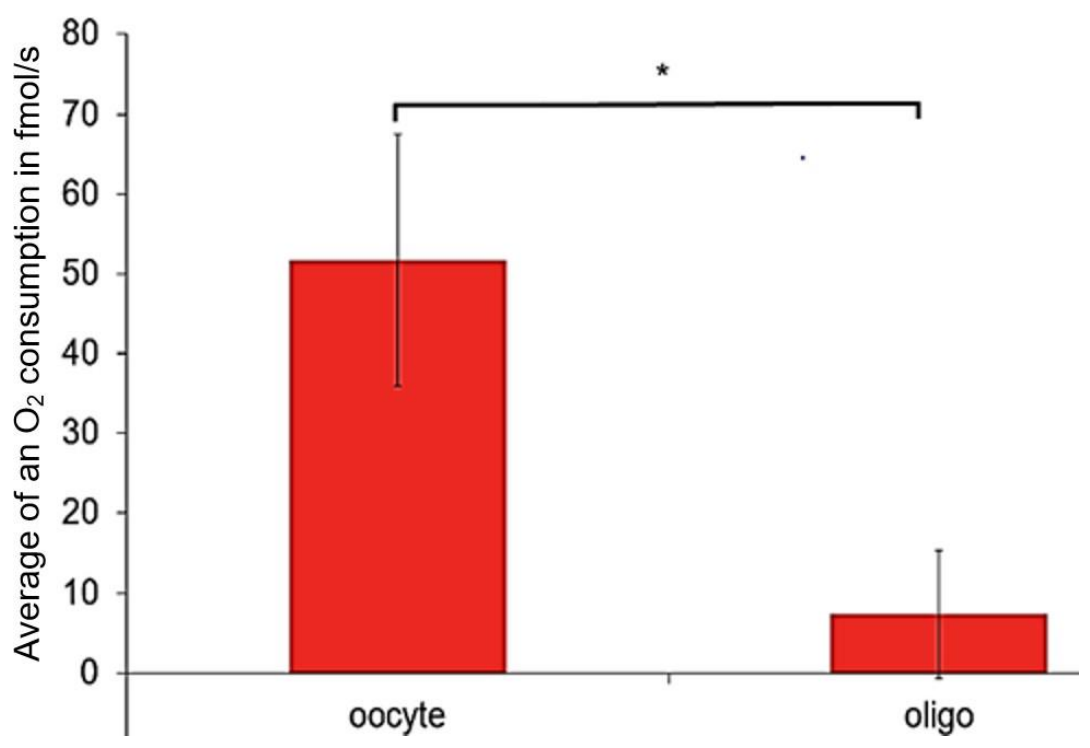

Fig. 4.10 Oxidative phosphorylation activity of equine oocytes: Basal respiration and the amount of respiration after adding oligomycin (error bars are SD of the measurements of 7 cells). * paired t-test: $p<0.01$. 


\subsubsection{Effect of FCCP Titration on Cell Respiration of Bovine Oocytes and Embryos}

Importantly, basal oocyte respiration might not represent the cellular mitochondrial respiratory capacity, which can be limited by the basal activity of the ATP synthase (i.e., cellular energy demand). Determining the maximal mitochondrial respiratory capacity is useful for estimating metabolic potential and, when expressed in relation to basal respiration, an index of cellular metabolic activity.

Low concentrations of the protonophore "uncoupler" FCCP (1-2 $\mu \mathrm{M})$ are routinely used to reveal the maximal respiratory capacity of a cell by removing the limitation of electron flow imposed by mitochondrial membrane potential (proton gradient). However, slightly more FCCP (3-4 $\mu \mathrm{M})$ may inhibit respiratory chain enzymes and decrease respiration in many cell types, resulting in erroneous estimates of cellular respiratory capacity [96]. This effect had not been previously investigated in oocytes, so we performed $1 \mu \mathrm{M}$ titrations of FCCP following stabilization basal oocyte respiration.

The effect of four $1 \mu \mathrm{M}$ titrations of FCCP on bovine oocyte respiration was observed and the result is shown in Fig. 4.11A. FCCP titrations was done after (20-30) min basal respiration and the effect of each titration was measure for $10 \mathrm{~min}$. As expected, the results demonstrate the sensitivity of the electrode to pinpoint the maximal rate of oocyte respiration following $\sim 2 \mu \mathrm{M}$ FCCP, after which an inhibitory effect becomes evident.

Fig. 4.11B shows that the maximal respiration in bovine embryos is achieved between 1-2 $\mu \mathrm{M}$ FCCP, indicating potential variations in the optimal concentration of FCCP needed to obtain the true maximal respiratory capacity between cell types or developmental stage. 

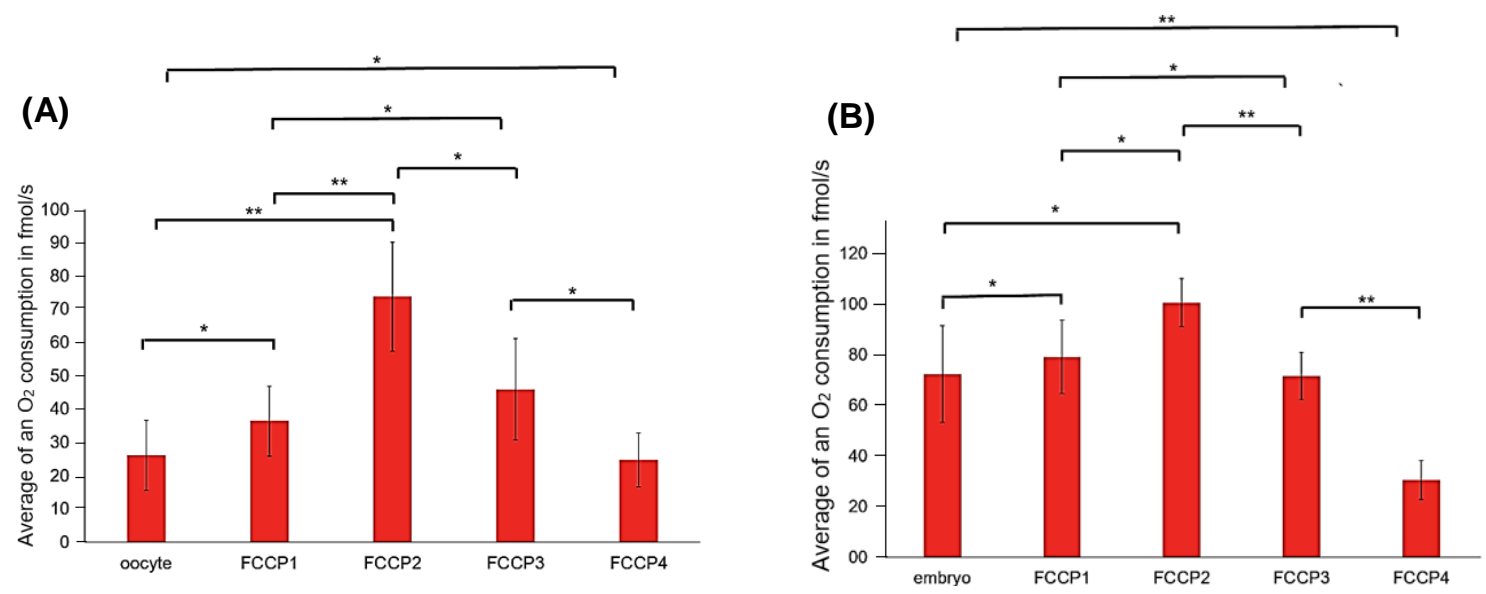

Fig. 4.11. (A) OXPHOS of bovine oocytes: Basal respiration and the respiration after four sequential additions of $1 \mu \mathrm{M}$ FCCP (mean $\pm S D, n=10$ ) (B) OXPHOS of bovine embryos: Basal respiration and the amount of respiration after 4 times titration of $1 \mu \mathrm{M} \mathrm{FCCP} \mathrm{(mean \pm SD,} n=6$ ). *paired t-test: $\mathrm{p}<0.05$; ** paired t-test: $\mathrm{p}<0.01$.

\subsubsection{Basal Respiration and the Effect of Oligomycin and FCCP Titration on Bovine Oocytes}

Based on the findings in Fig. 4.11, we repeated the experiments to test the effect of oligomycin and FCCP titration on bovine oocytes and embryos. The results of basal respiration and expected inhibitory effects of adding oligomycin $(1 \mu \mathrm{M})$, and recovery of respiration with three $1 \mu \mathrm{M}$ titrations of FCCP on single bovine oocytes and embryos are shown in Fig. 4.12A and Fig.

4.12B, respectively.

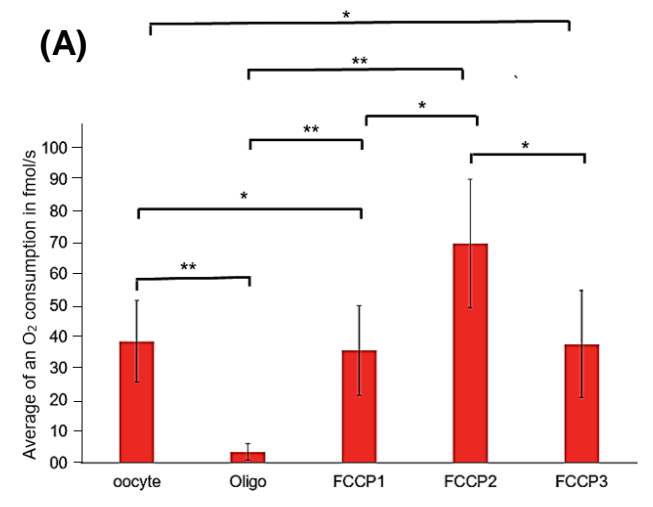

(B)

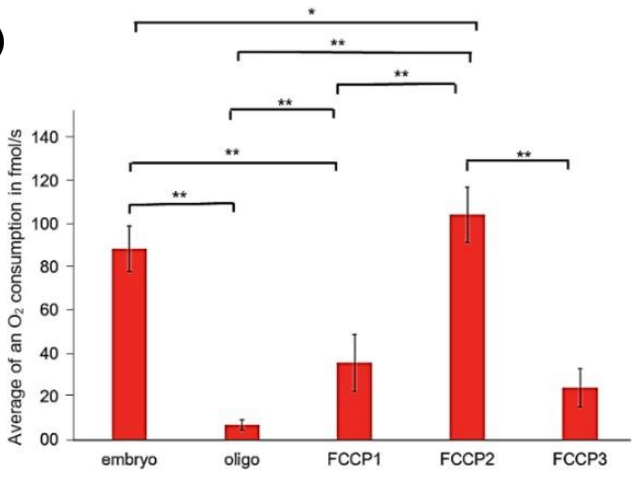

Fig. 4.12. (A) OXPHOS of bovine oocytes: Basal respiration and the respiration after adding oligomycin and 3 additive titrations of $1 \mu \mathrm{M}$ FCCP (mean $\pm S D, n=8$ ). (B) OXPHOS of bovine embryos: Basal respiration and the amount of respiration after adding oligomycin and 3 additive titrations of $1 \mu \mathrm{M}$ FCCP (mean $\pm S D, n=10$ ). " paired t-test: $p<0.05$; ** paired t-test: $p<0.01$. 


\subsubsection{Discussion}

This sensor is capable to basal cell respiration supported by endogenous substrates, and respiration associated with proton leak induced by inhibition of the adenosine triphosphate (ATP) synthase (complex $\mathrm{V}$ ) with oligomycin, and the maximal non-coupled respiratory capacity revealed by Carbonyl cyanide-4-(trifluoromethoxy)phenylhydrazone (FCCP) titration. Validation that respiration measurements were indeed cellular oxidative phosphorylation was demonstrated by expected responses to ATP synthase inhibition and the induction of maximal respiration by titration of the protonophore FCCP. Although, one other study has demonstrated the importance of carefully titrating micromolar concentrations of FCCP to avoid its inhibitory effects on respiration in other cell types [96], Nearly all papers utilizing commercially available systems (e.g., Seahorse Extracellular Flux Analyzer) report experiments where only a single FCCP concentration is employed (e.g., $1.5 \mu \mathrm{M}$ ) [97], which could underestimate the true maximal respiratory capacity. Similarly, in the paper by Sugimura et al., only a single FCCP concentration was used $(2 \mu \mathrm{M})$ in their SECM assays [95]. To our knowledge, our work is the first to demonstrate the importance of titrating FCCP to determine the true maximal respiratory capacity of intact respiring oocytes specifically showing that both $1 \mu \mathrm{M}$ is insufficient, and $3 \mu \mathrm{M}$ becomes inhibitory, and thus both underestimate the maximal respiratory capacity.

The range of respiration rate at a single cell level previously reported varies between $\mathrm{fmol} / \mathrm{s}$ to $\mathrm{pmol} / \mathrm{s}[97,95-99]$. However, it should be taken into account that different types of cells have different behaviors and different respiration measurements, the readings that were obtained in this work for bovine/equine oocytes or bovine embryos basal respiration are all in $\mathrm{fmol} / \mathrm{s}$ and they are comparable with the range found in the literature. The respiration rates reported by our work are slightly higher than what was previously reported in the literature. This can be resulted from the method of calculating the respiration rate. In our work, the respiration was measured at the 
vicinity of the cell and no concentration gradient was used in the calculation because of the small volume of the medium that we used.

Finally, our sensor was able to detect the effect of adding oligomycin and FCCP on the cell respiration rate. As was shown in the results section, the addition of oligomycin reduced respiration of the cell to about $1 / 7$ of it is basal respiration rate. This is better than what was reported by Sugimura et al. were only able to get a reduction of about half the basal respiration when Oligomycin was added [96], whereas in [80] by Santos et al., their system was able to detect a reduction of a cell respiration to $1 / 3$ of it is basal respiration rate. For related FCCP effects, the maximum value of respiration rate measured after the second FCCP titration was used to compare with what was reported in $[96,100]$. Our results have shown that the cell respiration rate increases to about double of its basal respiration rate after adding FCCP and that was approximately same amount of increase reported in [96, 100]. 


\section{Chapter 5: A Multi-Sensor System for Measuring Bovine Embryo Metabolism}

\subsection{Background of the System}

A multi-sensor platform capable of simultaneous measurement of DO concentration, glucose and lactate concentrations in a micro-chamber for real-time evaluation of metabolic flux in bovine embryos was developed. A micro-chamber containing all three sensors (DO, glucose, and lactate) was made to evaluate metabolic flux of single oocytes or embryos at different stages of development in $\leq 120 \mu \mathrm{L}$ of respiration buffer. The ability of the sensor to detect a metabolic shift from oxidative phosphorylation (OXPHOS) to glycolysis was demonstrated in embryos by an ablation of oxygen consumption and an increase in lactate production following addition of oligomycin. An increased reliance upon glycolysis relative to OXPHOS was demonstrated in embryos as they developed from morula to hatched blastocysts by a progressive increase in the lactate/oxygen flux ratio, consistent with isolated metabolic assessments reported previously. These studies highlight the utility of a metabolic multi-sensor for integrative real-time monitoring of aerobic and anaerobic energy metabolism in bovine embryos, with potential applications in the study of metabolic processes in oocyte and early embryonic development. The details of this chapter are largely based on a published journal paper, "A Multi-Sensor System for Measuring Bovine Embryo Metabolism [101]".

\subsection{Materials and Methods}

\subsubsection{Materials and Reagents}

SU8-2050 and SU8 developer were purchased from MicroChem Corp (MA, USA). Megaposit MF -26A developer and S1813 photoresist were purchased from Capitol Scientific, Inc (Austin, TX). Glass substrates, $5 \% \mathrm{w} / \mathrm{w}$ Nafion perfluorinated resin, glucose oxidase (GOx), lactate oxidase (LOx), D- (+)-glucose, sodium L-lactate, phosphate buffer saline (PBS), Tween-20, oligomycin, and bovine serum albumin (BSA) were purchased from Sigma Aldrich (St. Louis, 
Missouri). Sodium sulfite $\left(\mathrm{Na}_{2} \mathrm{SO}_{3}\right)$ was purchased from Eisen-Golden laboratories (Berkeley, California). G-MOPS medium (respiration buffer) and paraffin oil (OVOIL ${ }^{\mathrm{TM}}$ ) were purchased from Vitrolife.

\subsubsection{Electrodes and Chamber Designs and Manufacturing}

A multi-sensor chip was fabricated on a glass substrate of $24.5 \mathrm{~mm} \times 24.5 \mathrm{~mm}$ through standard photolithography and lift-off techniques. The three-electrode configuration with working electrode (WE), quasi reference electrode (RE), and counter electrode (CE) was used for all sensor configurations. A $12 \mathrm{~mm}$ diameter and $3 \mathrm{~mm}$ deep micro-chamber containing all three sensors was made using SU8. A glass lid with two drilled holes (inlet and outlet) was glued on the chamber (Fig. 5.1).

\subsubsection{Photolithography}

The multi-sensor chip was fabricated on a glass substrate of $24.5 \mathrm{~mm} \times 24.5 \mathrm{~mm}$ through standard photolithography and lift-off techniques. A mask was designed using AutoCAD® software (Autodesk, Inc.) which was used for generating electrodes patterns.

The photolithography steps include cleaning the substrate by acetone, methanol and DI water. The substrate was dried using nitrogen $\left(\mathrm{N}_{2}\right)$ and baked at $135^{\circ} \mathrm{C}$ to remove water from the surface. $\mathrm{S} 1813$ photoresist was spin-coated at a low speed of 700 revolution per minute (rpm) for 5 seconds (s) and at a high speed of $3000 \mathrm{rpm}$ for $30 \mathrm{~s}$. The chips were baked at $135{ }^{\circ} \mathrm{C}$ for 1 minute ( $\mathrm{min}$ ); the electrodes mask was aligned on the chip and exposed to a 20-Watt UV light source of 395-405 nm wavelength range for $13 \mathrm{~s}$. The exposed chips were immersed in Megaposit MF -26A developer for one min, and finally immersed in DI water bath for 1 min and dried using nitrogen $\left(\mathrm{N}_{2}\right)$. 


\subsubsection{Metal Deposition}

A $20 \mathrm{~nm}$ of Chromium (Cr) was first evaporated onto the substrate as an adhesion layer, followed by the evaporation of $100 \mathrm{~nm}$ gold $(\mathrm{Au})$ layer onto the substrate. A liftoff process was performed afterwards to form the individual electrodes on the substrate. The sensor sizes are $0.368 \mathrm{~mm}^{2}$ for the WE, $0.259 \mathrm{~mm}^{2}$ for the $\mathrm{CE}$, and $0.169 \mathrm{~mm}^{2}$ for the quasi RE.

\subsubsection{Micro-Chamber}

A $3 \mathrm{~mm}$ deep SU8 micro-chamber was made using photolithography. The photolithography steps include cleaning the substrate by acetone, methanol and DI water. The substrate was dried using $\mathrm{N}_{2}$ and the chip was placed on a hot plate of $80{ }^{\circ} \mathrm{C}$; SU8-2050 was dripped on the designed chip, then the layer of photoresist was distributed evenly after $2 \mathrm{~s}$. Bubbles formed on the surface of the photoresist layer were removed using a pipette. The chip was placed on the hotplate for 30 min to soft bake the SU8 layer, and a lid was used to cover the chip during baking to prevent solvent evaporation and cracking. After the soft bake, the chip was cooled down to room temperature for $10 \mathrm{~min}$. Micro-chamber masks were used to expose the soft-baked SU8 layer to UV for 10 min, followed by a 30-min post exposure bake with a lid covering the chip to prevent solvent evaporation. The chip was cooled at room temperature for $15 \mathrm{~min}$, immersed in a developer for 1 hour (h), then rinsed using isopropanol and dried with $\mathrm{N}_{2}$.

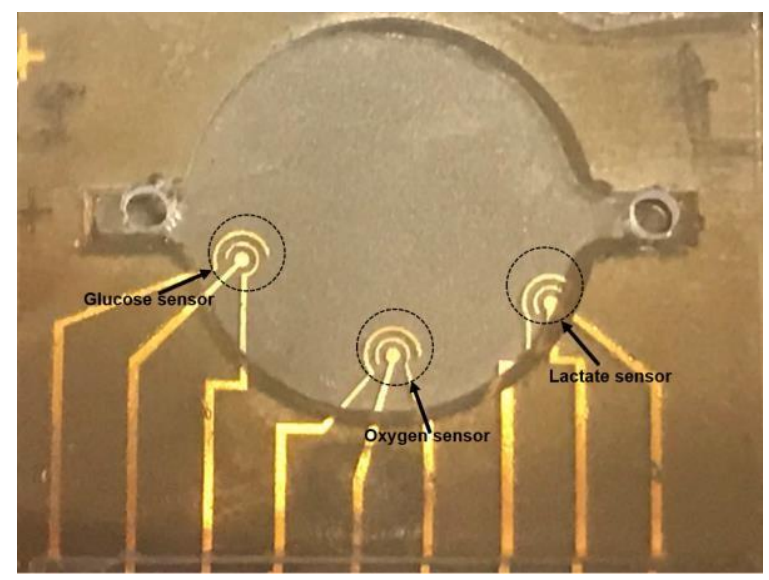

Fig. 5.1. Complete design with $3 \mathrm{~mm}$ thick SU8 micro-chamber covered by a glass lid. 


\subsubsection{Sensors Surfaces Modification}

\subsubsection{Oxygen Electrode Surface Modification}

Among different surface preparation methods, Nafion was chosen as a solid-state electrolyte as well as a membrane. Using Nafion only is more compatible with integration and multiplexed sensing applications than using other electrolytes and membranes [50]. A solid electrolyte layer was formed on the electrode surface by applying $0.1 \mu \mathrm{L}$ of Nafion solution to the surface of the WE and allowing it to dry for 20 min.

\subsubsection{Glucose Electrode Surface Modification}

The GOx film solution was prepared by dissolving $5 \mathrm{mg}$ of GOx and $50 \mathrm{mg}$ of BSA in $500 \mu \mathrm{L}$ of $1 \mathrm{mM}$ PBS containing $0.02 \% \mathrm{v} / \mathrm{v}$ Tween-20. BSA was used to allow better immobilization of the enzymes on the electrode surface. The glucose WE was covered by $0.1 \mu \mathrm{L}$ of the GOx solution and was left to dry for $30 \mathrm{~min}$ at room temperature. Then a $0.1 \mu \mathrm{L}$ of $5 \% \mathrm{w} / \mathrm{w}$ Nafion was used to cover the GOx film surface before drying for $15 \mathrm{~min}$ at room temperature. The functionalized electrodes were refrigerated $\left(4^{\circ} \mathrm{C}\right)$ in phosphate buffer until use.

\subsubsection{Lactate Electrode Surface Modification}

The LOx film solution was prepared by dissolving $2.5 \mathrm{mg}$ of LOx and $50 \mathrm{mg}$ of BSA in 500 $\mu \mathrm{L}$ of $1 \mathrm{mM}$ PBS containing $0.02 \% \mathrm{v} / \mathrm{v}$ Tween-20. The lactate electrode was covered by LOx film by adding $0.1 \mu \mathrm{L}$ of the LOx solution to the WE surface and was left to dry for 30 min at room temperature. Nafion was diluted with ethanol, with one-part Nafion to nine parts ethanol. Then a $0.1 \mu \mathrm{L}$ of Nafion was added to the LOx film surface and allowed to dry for 15 min at room temperature. The functionalized electrodes were placed in a refrigerator $\left(4^{\circ} \mathrm{C}\right)$ in phosphate buffer until use.

\subsubsection{Bovine Embryos and Their Stimuli}

Bovine oocytes were obtained from ovaries collected at a slaughterhouse and fertilized and incubated in embryo culture (G-MOPS) at $38.5^{\circ} \mathrm{C}$ (see Section 4.4.1). Oligomycin $(1 \mu \mathrm{M})$ was 
used as an ATP synthase inhibitor. The initial volume of the respiration medium used in all experiments was $120 \mu \mathrm{L}$. Stock solutions of oligomycin were made up in $100 \%$ ethanol to provide $1 \mu \mathrm{M}$ concentration in the $120 \mu \mathrm{L}$ respiration chamber with a $1 \mu \mathrm{L}$ volume.

\subsubsection{Electrochemical Instrumentation}

A potentiostat (eDAC, Quadstat EA164H, Colorado Springs, CO) was used to perform all electrochemical measurements. Data collected using potentiostat were analyzed using a set of custom-built tools and Gui written in MATLAB (The MathWorks, Inc.) (Appendix F) for data calibration and conversion from $\mu \mathrm{A} / \mathrm{min}$ to the equivalent respiration rate in $\mathrm{fmol} / \mathrm{s}$.

\subsubsection{Sensors Activation Voltages}

The range of activation voltages for $\mathrm{DO}$, glucose, and lactate have been reported [40, 43, 9091]. However, the actual activation voltage also depends on other sensor features such as electrode geometry and electrode surface preparation.

\subsubsection{Oxygen Sensor Activation Voltage}

To determine the required activation voltage for measuring DO using the sensor in this chapter, CV was performed. DI water was used as saturated oxygen solutions, while sodium sulfite was used as a zero-oxygen solution. The potential was swept from $-1.5 \mathrm{~V}$ to $2 \mathrm{~V}$ at 100 $\mathrm{mV} / \mathrm{s}$ with respect to $\mathrm{Au}$ quasi-reference electrode. And a noticeable reduction current was observed in the range $-0.55 \mathrm{~V}$ to $-0.7 \mathrm{~V}$ (Fig. 5.2). To achieve the least interference from other molecules in the solution, low voltage is preferred; in all experiments, a reduction voltage of -0.6 $\mathrm{V}$ was used.

\subsubsection{Glucose Sensor Activation Voltage}

The range of activation voltages in the literature for measuring lactate and glucose depends on the electrode surface modifications and enzymes layers used [12, 40, 43]. To determine the required activation voltage for measuring glucose, CV was performed where the potential was 
swept from $-1 \mathrm{~V}$ to $1 \mathrm{~V}$ at $100 \mathrm{mV} / \mathrm{s}$ with respect to Au quasi-reference electrode. When $10 \mathrm{mM}$ glucose solution was tested, a noticeable current peak was observed at $0.4 \mathrm{~V}$ (Fig. 5.3A).

\subsubsection{Lactate Sensor Activation Voltage}

The required potential for measuring lactate was determined by CV with the potential from -1 $\mathrm{V}$ to $1 \mathrm{~V}$ at $100 \mathrm{mV} / \mathrm{s}$ with respect to Au quasi-reference electrode. A current peak was detected at $0.4 \mathrm{~V}$ when $5 \mathrm{mM}$ lactate was measured (Fig. 5.3B).

\subsubsection{Hydrogen peroxide $\left(\mathrm{H}_{2} \mathrm{O}_{2}\right)$ Activation Voltage}

Glucose and lactate are measured indirectly by amperometric oxidation of hydrogen peroxide, which is produced from oxidizing of glucose and lactate at the electrodes containing their respective enzymes $[12,68]$. The required potential for measuring glucose or lactate is basically the potential needed to measure the hydrogen peroxide generated. Therefore, a CV test for $10 \mathrm{mM}$ hydrogen peroxide was done (Fig. 5.3C).

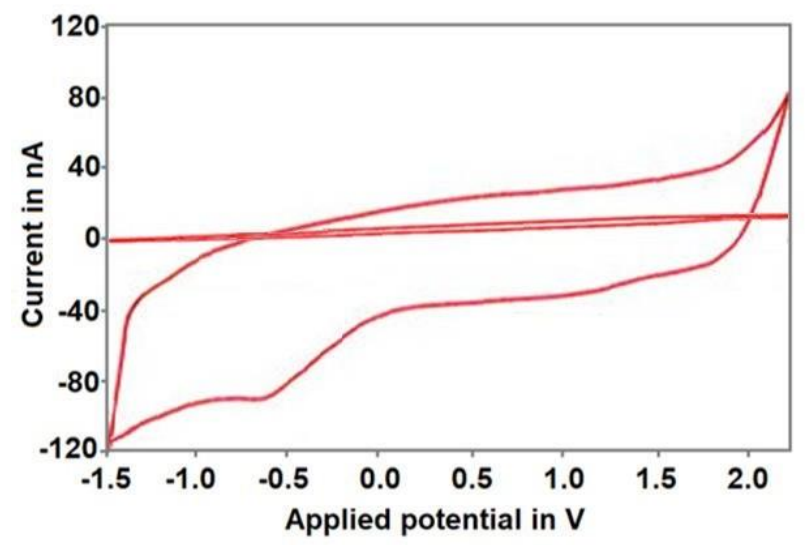

Fig. 5.2. CV of oxygen sensor when DI water was tested. 

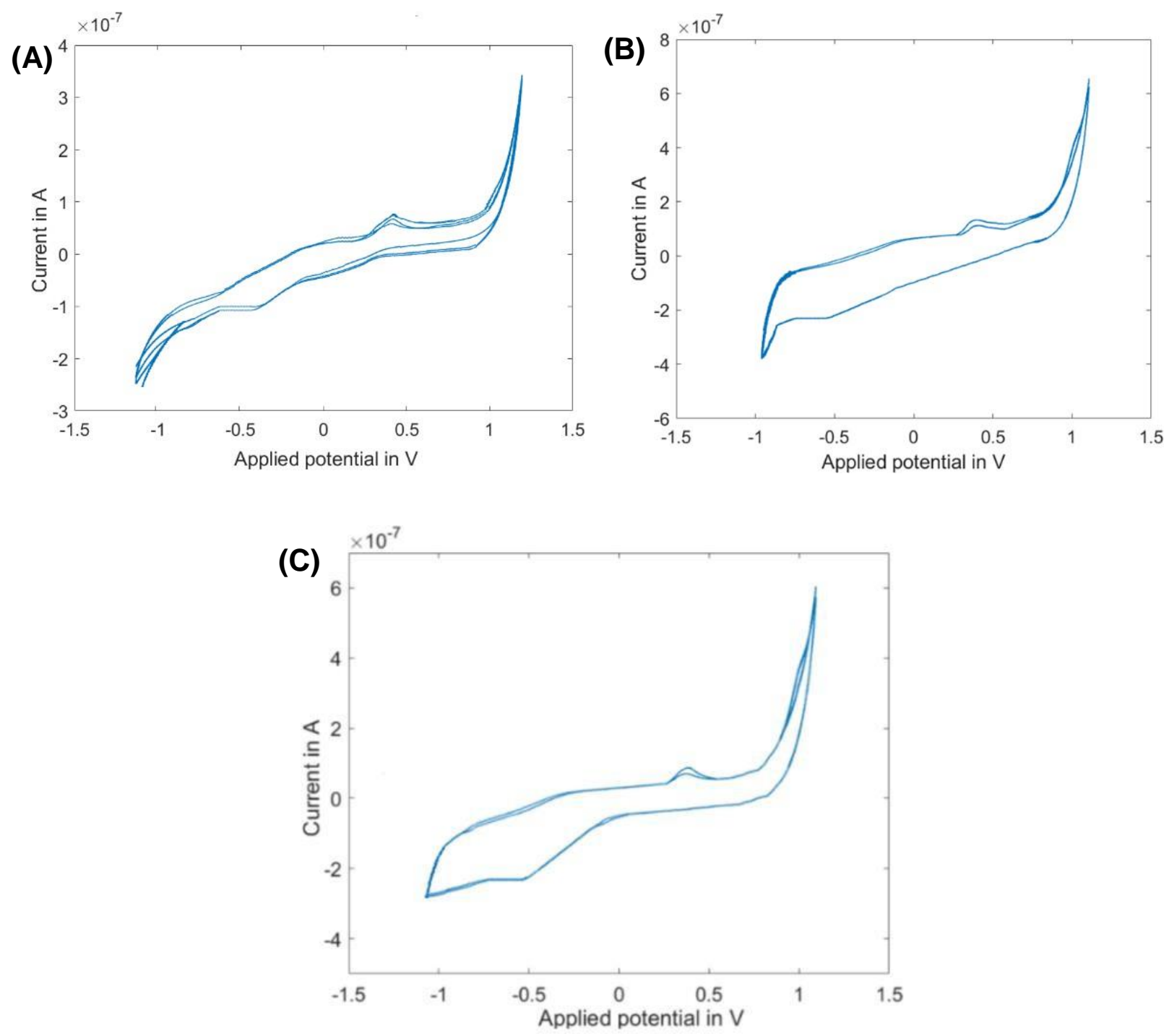

Fig. 5.3. (A) CV of glucose sensor. (B) CV of lactate sensor. (C) CV of hydrogen peroxide

\subsubsection{Sensors Calibration}

\subsubsection{Oxygen Sensor Calibration}

To determine the calibration curve and linearity of the DO sensor, the DO concentration was changed by adding $0.1 \mathrm{M} \mathrm{Na}_{2} \mathrm{SO}_{3}$ to the saturated solution in incremental steps with continuous stirring to produce different oxygen concentrations for generating the calibration data [89]. All $\mathrm{O}_{2}$ concentration measurements were made at $38.5^{\circ} \mathrm{C}$ and validated using a calibrated Oakton DO6+ DO meter. The DO reduction current was measured at $-0.6 \mathrm{~V}$ at $0.5 \mathrm{~min}$ after each addition of 
$\mathrm{Na}_{2} \mathrm{SO}_{3}$. The DO concentration in the G-MOPS respiration buffer corresponding to the measured current in the calibration curve was calculated at $158 \mu \mathrm{M}$ based on the average barometric pressure of the experiment location (Fort Collins, Colorado $(84.8 \mathrm{kPA})$ ) at the temperature of $38.5^{\circ} \mathrm{C}$, corrected for the slightly lower oxygen solubility of the G-MOPS respiration buffer $(0.92)$ compared to water [92].

\subsubsection{Lactate Sensor Calibration}

A $10 \mathrm{mM}$ lactate solution was prepared using L-lactate powder taking into consideration its molecular weight (112.06 gram/mole) and calculations required to convert grams into $\mathrm{mol} / \mathrm{L}$. Solutions with different lactate concentrations were prepared by diluting a known lactate solution in PBS buffer. The current generated from the oxidation of hydrogen peroxide was measured at $0.4 \mathrm{~V}$ at $38.5^{\circ} \mathrm{C}$.

\subsubsection{Glucose Sensor Calibration}

A $20 \mathrm{mM}$ glucose solution was prepared using D-glucose powder, taking in considering its molecular weight (180 gram/mole) and calculations required to convert grams into mol/L. Different glucose concentrations solutions were prepared by diluting a known glucose concentration solution in PBS buffer. The current generated from the oxidation of hydrogen peroxide was measured at $0.4 \mathrm{~V}$ at $38.5^{\circ} \mathrm{C}$.

\subsubsection{Measurement Setup}

\subsubsection{Oxygen, Glucose and Lactate Measurements}

The multi-sensors chip embedded in the micro-chamber described in Section 5.2.2 was used. The temperature of the medium was maintained at $38.5^{\circ} \mathrm{C}$ by placing the device on a stage warmer for a stereomicroscope. The applied potential during all the amperometric experiments was set at $-0.6 \mathrm{~V}$ for measuring $\mathrm{DO}$ and at $0.4 \mathrm{~V}$ for measuring lactate and glucose. The electrodes were rinsed using DI water and electrochemically pulse cleaned before and after each test. Sterilized water was used for pulse cleaning to avoid any toxic effect on cells from any other 
cleaning chemicals. The pulses used for cleaning were $1.2 \mathrm{~V}$ peak-to-peak with a scan rate of 50 $\mathrm{mV} / \mathrm{s}$ for $1 \mathrm{~min}$.

G-MOPS respiration buffer $(120 \mu \mathrm{L})$ was first placed in the micro-chamber and overlaid with $120 \mu \mathrm{L}$ of paraffin oil to seal the micro-chamber. The intrinsic oxygen, glucose, and lactate concentrations in the buffer were assessed, and the measured currents were used as baselines to determine oxygen consumption, glucose consumption, and lactate production after adding embryos. A technician selected and moved the embryos into the micro-chamber, and the readings were done with the sensor operator blinded to the quality or type of sample. Embryos of different stages were tested during these experiments. A single embryo was transferred into the microchamber by pipetting through the paraffin oil layer on top of the WE, while viewing through a stereoscope. Analytes were measured for 8-10 min each by reading DO, glucose, and lactate consecutively, with the embryo moved from one WE to another for specific analyte reading.

\subsubsection{Model Used for Analyte Consumption/Production Calculations}

Oxygen consumption was measured by monitoring the decrease in the oxygen reduction current over time during cell respiration, glucose consumption was measured by monitoring the decrease in the $\mathrm{H}_{2} \mathrm{O}_{2}$ oxidation current over time during cell respiration, and lactate production was measured by monitoring the increase in the $\mathrm{H}_{2} \mathrm{O}_{2}$ oxidation current over time during cell respiration. The change in the measured current over a given period of time was converted to its equivalent analyte concentration change within a defined volume in fmol/s using the calibration curve of the sensor and a similar model used in Section 4.4.4.

Assuming the concentration gradients of analytes within the micro-chamber towards the cell reached its equilibrium state when measurements were taken (i.e., changes in measured currents over time are constants), the defined volume is the volume immediately surrounding the cell inside the micro-chamber. Cell heights ranged from 120-250 $\mu \mathrm{m}$, and the cell is placed on the WE during experiments. The defined volume for analytes consumption/production calculations is the area of 
the WE multiplied by the height of (170-300) $\mu \mathrm{m}$. The height was chosen by considering the cell height range and another 50-100 $\mu \mathrm{m}$ above the cell which is the volume immediately surrounding the cell. This assumption was used to convert analytes uptake/release from current readings to change in concentrations in fmol/s. Therefore, Analyte uptake/release in $\mathrm{fmol} / \mathrm{s}(\Delta \mathrm{C}(\mathrm{fmol} / \mathrm{s}))$ is defined as:

$$
\Delta C\left(\frac{f m o l}{s}\right)=\frac{\Delta C}{\Delta t} x V
$$

where $\Delta \mathrm{C} / \Delta \mathrm{t}$ is the change in concentration over a given time period and $\mathrm{V}$ is the defined volume within the micro-chamber. Eq. $\mathbf{5 . 1}$ can be rewritten as:

$$
O C\left(\frac{f m o l}{s}\right)=\frac{\Delta I / \Delta t}{\Delta I / \Delta C} \times V
$$

where $\Delta \mathrm{l} / \Delta \mathrm{t}$ is the change of reduction or oxidation current over a given time obtained during experiments, and $\Delta \mathrm{l} / \Delta \mathrm{C}$ is the calibration curve.

The goal of the model represented in Eq. $\mathbf{5 . 2}$ is to report the analytes consumption/production rates in the immediate vicinity of the cell. Since the cell is located on top of the sensor, the model presented by Eq. 5.2 is the average rate directly obtained by the difference over a unit time of sensor readings (WE electrode) in its immediate vicinity. The choice of height range for calculating the volume may seem to be a bit arbitrary. However, this choice itself is not that important for the study presented in this paper since we report concentrations changes over a unit time period (i.e. consumption rate or production rate). A different choice of the height for volume calculation will give us a slight static bias on the measured rates. However, it will not alter changes of the measured rates under various stimuli. 


\subsection{Results and Discussion}

\subsubsection{Sensors Calibration}

The calibration curves for all sensors are shown in Fig. $\mathbf{5 . 4}$ and explained in the following sections.
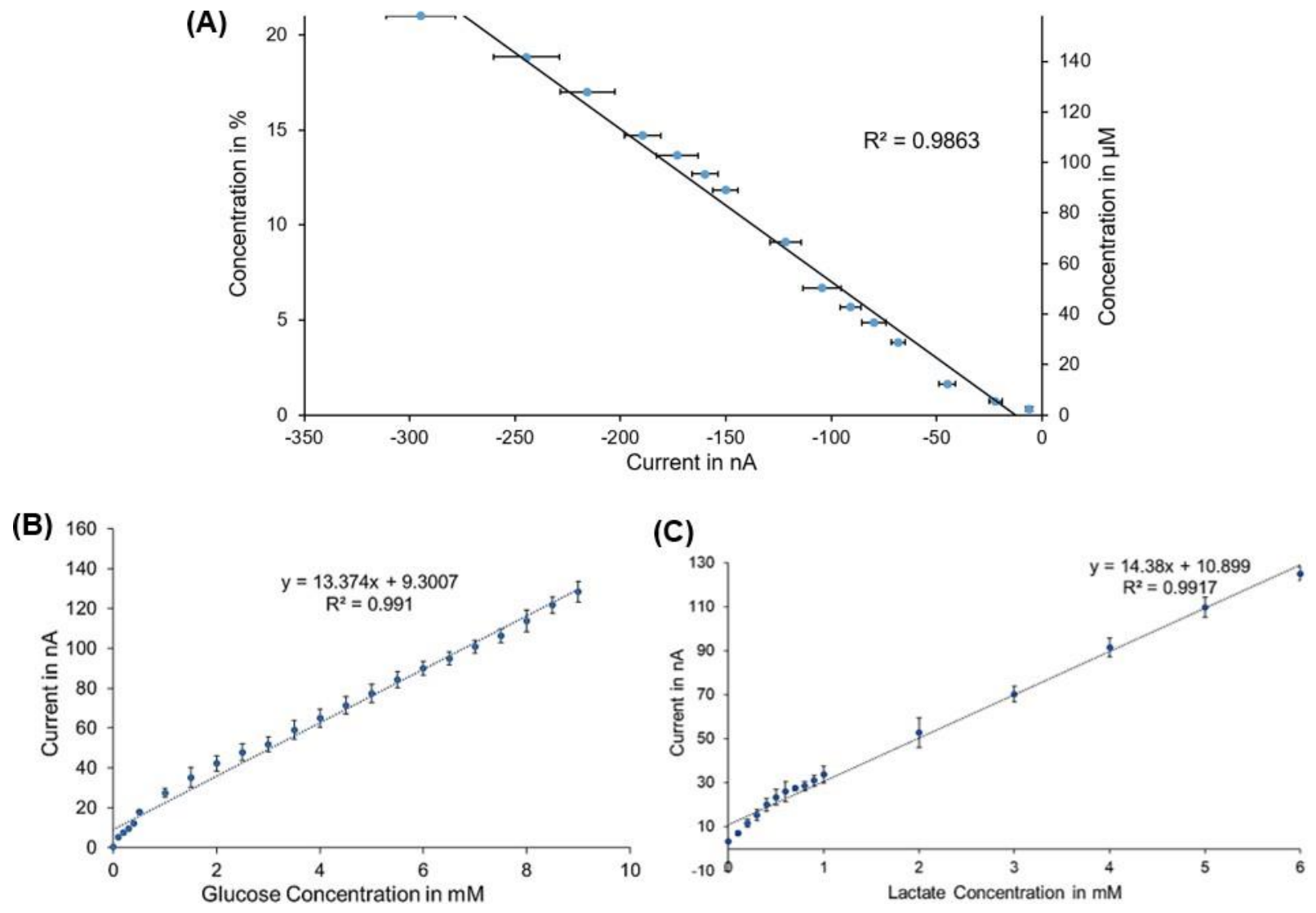

Fig. 5.4. (A) Oxygen sensor calibration curve: left y-axis represents concentration in \%, right yaxis is concentration in $\mu \mathrm{M}$. (B) Glucose sensor calibration curve. (C) Lactate sensor calibration curve. (mean $\pm \mathrm{SD}, \mathrm{n}=6$ )

\subsubsection{Oxygen Sensor Calibration}

A calibration curve (Fig. 5.4A) was obtained for the oxygen sensor, which demonstrated its ability to measure a DO range of 0 to $170 \mu \mathrm{M}$, with a sensitivity of $1.93 \mathrm{nA} / \mu \mathrm{M}(13.9 \mathrm{nA} / \%)$. Sensor output current increased linearly with the increase in $\mathrm{O}_{2}$ concentration with a correlation coefficient of 0.986 and had a limit of detection (LOD) of $1.22 \mu \mathrm{M}$. 


\subsubsection{Glucose Sensor Calibration}

The calibration curve generated for the glucose sensor (Fig. 5.4B) demonstrated a wide dynamic range of 0 to $9 \mathrm{mM}$ with good sensitivity (between 12.89 and $14.1 \mathrm{nA} / \mathrm{mM}$ ) and linearity $(r=0.98-0.99)$, and a LOD of $0.5 \mu \mathrm{M}$.

\subsubsection{Lactate Sensor Calibration}

The calibration curve (Fig. 5.4C) obtained for the lactate sensor demonstrated a wide dynamic range of 0 to $6 \mathrm{Mm}$ with good sensitivity $(12.1-14.38 \mathrm{nA} / \mathrm{Mm})$ and linearity $(r=0.99)$, and a LOD of $0.4 \mu \mathrm{M}$.

\subsubsection{Characterization of Bovine Embryo Energy Metabolism}

\subsubsection{Effect of Oligomycin on Embryo Metabolism}

To determine the specific ability of the multi-sensor to monitor a metabolic shift from OXPHOS to glycolysis in real-time, the metabolism of 6 embryos (at the blastocyst stage) were measured before and after adding oligomycin. Oligomycin inhibits the mitochondrial ATP synthase by blocking $\mathrm{H}+$ transport through the complex, thereby forcing cells to rely on non-mitochondrial (primarily glycolytic) ATP production for survival [45]. Fig. 5.5A shows the averages of the oxygen and glucose consumption and lactate production before and after the addition of oligomycin.

As expected, oligomycin stopped oxygen consumption, but increased lactate production, without a significant effect on glucose uptake. To better describe this observed effect on cellular metabolism, we calculated the flux ratio of lactate production to glucose uptake before and after adding oligomycin (Fig. 5.5B). This ratio increased significantly $(P \leq 0.01)$ after the addition of oligomycin, demonstrating a greater rate of lactate release relative to glucose consumption after inhibition of mitochondrial ATP production, consistent with a "switch" from OXPHOS to glycolysis to maintain cellular ATP production. These results highlight that embryo glucose uptake alone is not a reliable measure of glycolytic energy production, since the pyruvate generated in glycolysis 
can be oxidized in mitochondria (glucose oxidation) or converted to and released as lactate (anaerobic glycolysis).
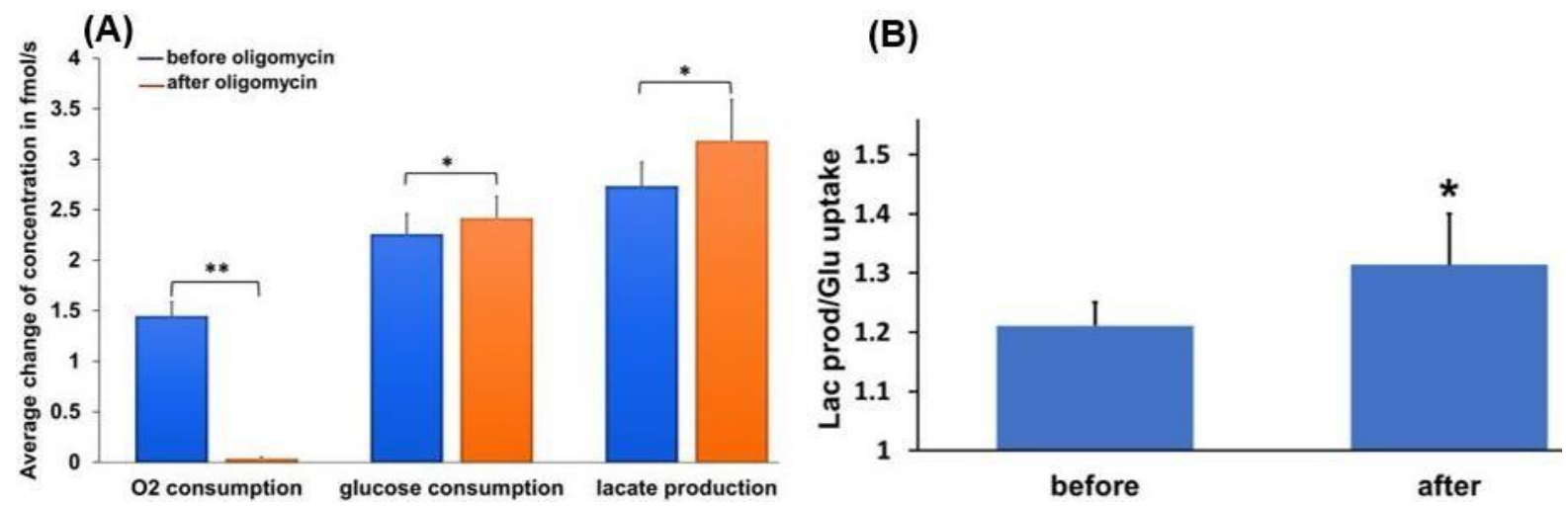

Fig. 5.5. (A) Oxygen and glucose consumptions and lactate production of bovine embryos before and after adding oligomycin. (B) Flux ratio (Lac prod/Glu uptake) of lactate production and glucose uptake for bovine embryos before and after adding oligomycin. (mean $\pm S D, n=6$ embryos at blastocyst stage). ${ }^{*}$ paired t-test: $P \leq 0.01,{ }^{* *}$ paired t-test: $P \leq 0.001$.

\subsubsection{Evaluation of Embryo Metabolism Throughout Development}

To investigate the metabolic characteristics of embryos during development, a total of 106 embryos in various stages were evaluated. Sixty-nine embryos graded good to excellent in quality (see B.1 in Appendix B), were used for metabolism studies. Glucose and oxygen consumption and lactate production are expressed as fmol per embryo per second \pm SD. The patterns of oxygen and metabolite flux during development from (8 to 32) cells to the hatched blastocyst stage was analyzed by one-way analysis of variance (ANOVA). Differences between means were examined using Tukey HSD (pairwise comparison among stages). Dead and degenerate oocytes and embryos (negative controls) had low measurements when compared with viable embryos of any development stage.

Throughout the observed stages of embryo development, every parameter of metabolic flux (oxygen, glucose, and lactate) significantly increased $(\mathrm{P}<0.001$, ANOVA). (See Table B.1 and Fig. B.2 in Appendix B for pairwise comparison) A non-significant change in mean oxygen consumption ( $P>0.05)$ was observed from early-stage embryos ( 8 to 32 cells) to morulae and 
from morulae to blastocysts (Fig. 5.6A) (See also Table B.1 and Fig. B.2 in Appendix B); but, oxygen uptake increased significantly from the blastocyst to expanding blastocyst stages and from expanding to hatching blastocyst stages $(P \leq 0.05)$. Glucose consumption followed a similar pattern to oxygen uptake with a significant increase from blastocyst to expanding blastocyst stages $(P \leq 0.05)$ and a highly significant increase from expanded to hatched blastocysts $(P \leq 0.05)$. Lactate production significantly increased from blastocyst to expanded blastocyst and from expanded to hatched blastocysts $(\mathrm{P}<0.05)$. (See Table B.1 and Fig. B.2 in Appendix B)

The observed increases in oxygen consumption, glucose uptake, and lactate production of bovine embryos as they progress toward the blastocyst stages are consistent with previous studies [98, 102], and parallel the well-established increase in embryo energy demand as cell numbers increase [103-104]. The mean values of glucose and lactate flux in bovine embryos in the present study are similar to those previously reported for bovine [98] and human embryos $[103,105]$. However, observed rates are lower than those reported for equine embryos [106] and bovine and human embryos incubated in lactate-free culture media [102, 107] suggesting variations due to both species and culture media composition. Indeed, levels of lactate may attenuate rates of glycolysis in vitro [108] and at least one previous study demonstrated that rate of glucose metabolism is linearly related to its concentration in the media [105]. The degree of increase in glucose consumption and lactate production by embryos from the morula to blastocyst stages in the present study is similar to previous studies utilizing glucose tracer methods in bovine embryos [109] and microfluorometry in equine embryos [106] but lower than some reports in bovine and human embryos $[98,102]$. These discrepancies could be explained in part using later stage (Day 6) morula in the present study which can behave similarly to blastocysts used in the latter studies [98, 106]. Table 1 provides a comprehensive comparison between the multi-sensor system described herein and the published results on methods, targeting species and cells, specific analytes to be measured, and the related sensor performance. 


\subsubsection{Metabolic Flux Ratios Reveal Shifts in Glucose Utilization during Embryo}

\section{Development}

A primary advantage of simultaneous measurement of metabolite flux using the multi-sensor system is its ability to express different rates relative to one another. This provides internallycontrolled indices of substrate utilization that are more sensitive than absolute flux rates of individual metabolites. Accordingly, to further investigate the nature of increased metabolic flux observed in embryos during development, we evaluated relative flux of lactate production/glucose uptake, the glucose uptake/oxygen uptake, and the lactate production/oxygen uptake at different stages of development (Figs. 5.6B, 5.6C, and 5.6D). Results show that while increases in all three flux ratios were observed (reflecting enhanced glucose metabolism), the amount of lactate produced relative to oxygen consumed nearly doubled from morula to hatched blastocyst stages $(P \leqslant 0.001)$, indicating an increasing contribution of anaerobic glycolysis to embryo ATP production during development. These findings are in general agreement with studies demonstrating increases in glucose and oxygen uptake and lactate production of bovine [102, 109], human [103, 105, 107], equine [106] and sheep embryos [110] at the expanding blastocyst stage.

Overall, the results of our studies are consistent with a link between embryo metabolic activity and development, and specifically a transition in the relative contribution of OXPHOS and glycolysis to energy production during later stages of development. However, as noted above, it is important to consider that embryos being studied in vitro are subject to the stress of being placed in an artificial environment, including the potential for nutrient imbalance and oxidative stress [2, 111], which have the potential to influence embryo genomic imprinting, development rate and metabolism [112-114]. Therefore, it is critical to understand how specific culture conditions (medium composition, length of exposure, developmental stage treatment applied, and concentrations of oxygen) interact and impact the metabolism of the preimplantation embryo, 
resulting in altered embryo and fetal development $[111,113,115]$. The use of metabolic multisensors such as the device described herein are ideally suited for characterization studies of this nature, which may ultimately help to optimize embryo incubation conditions and enhance the success rate of assisted reproduction technologies.

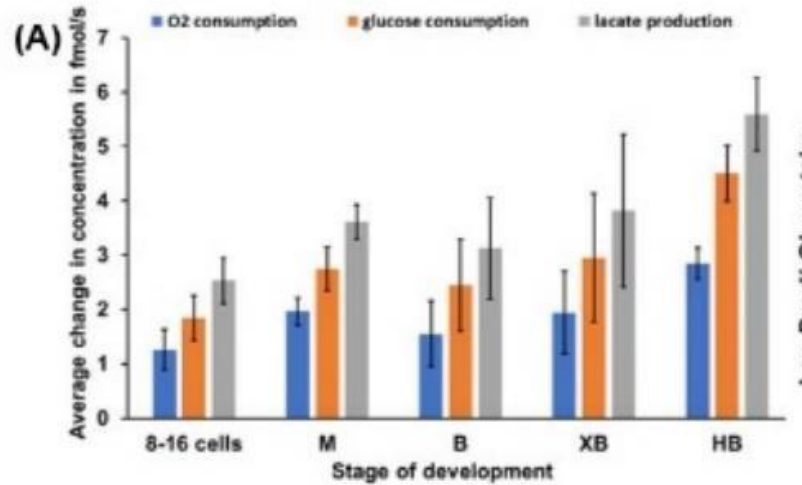

(B)

(C)
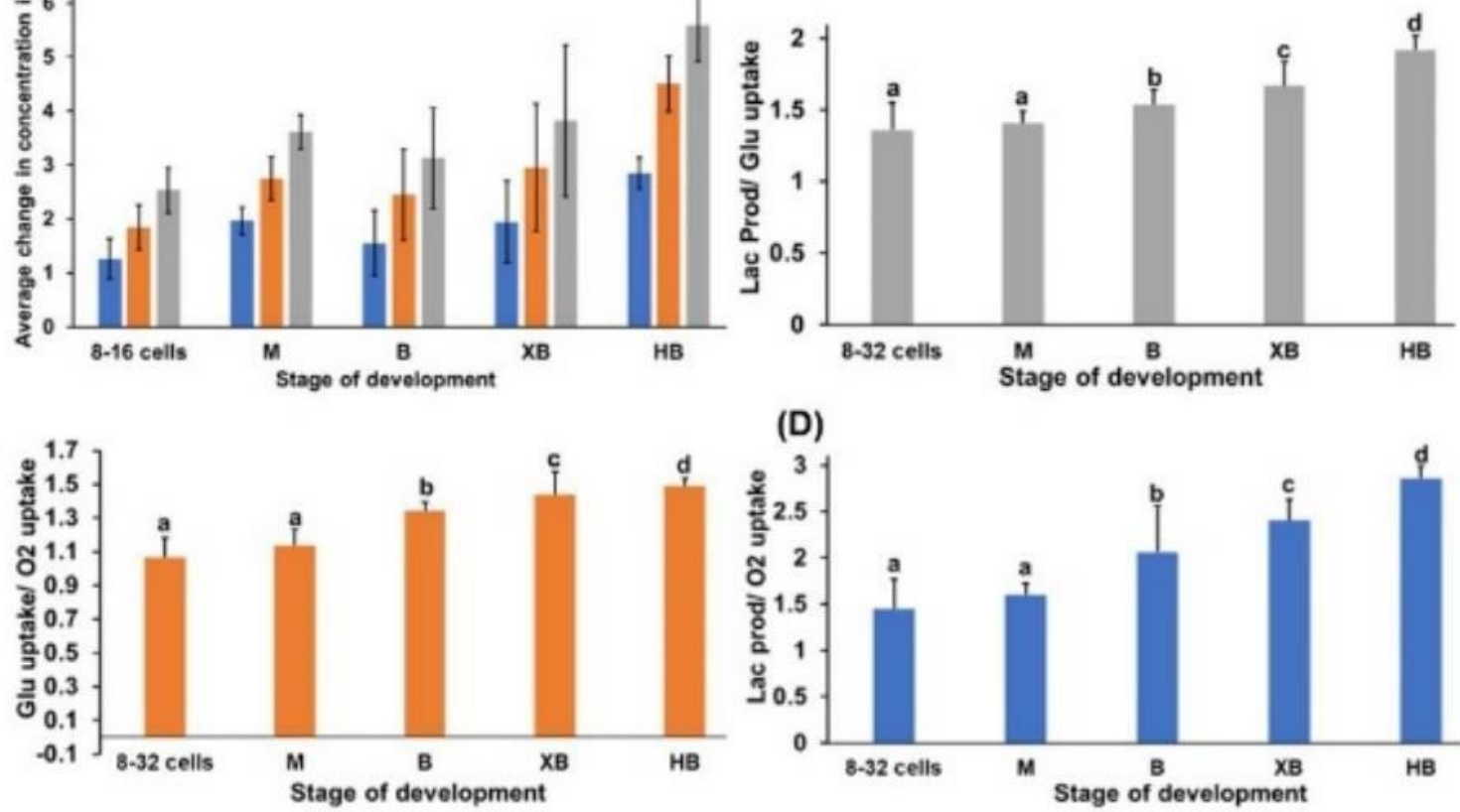

(D)

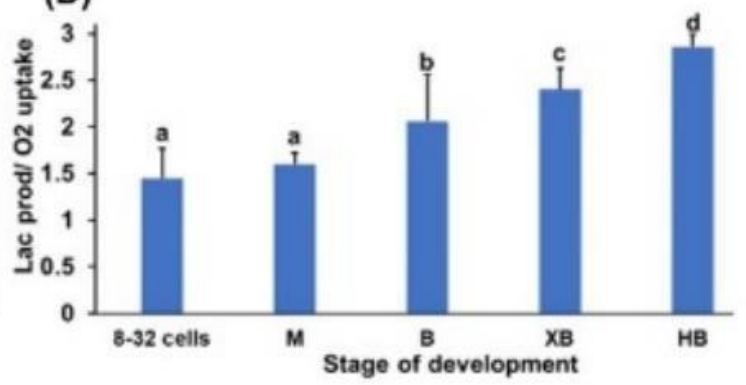

Fig. 5.6. (A) Oxygen and glucose consumptions and lactate production of bovine embryos at various stages. (B) Flux ratio between lactate production and glucose uptake (Lac prod/Glu uptake). (C) Flux ratio between glucose and oxygen uptakes (Glu uptake/ $\mathrm{O}_{2}$ uptake). (D) Flux ratio between lactate production and oxygen consumption (Lac $\mathrm{prod} / \mathrm{O}_{2}$ uptake). In all figures: mean $\pm S D$; dead oocytes or embryos $(n=12)$, 8 to 32 cells $(n=12)$, morula $(M, n=7)$, blastocyst $(B, n=6)$, expanded blastocyst $(X B, n=17)$, and hatched blastocyst $(n=8, H B)$. a, b, $c$, d within columns: Tukey HSD (pairwise comparison), values with different superscripts are significantly different $(P \leq 0.001)$.

\subsubsection{Advantages of the Present Design over Other Metabolism Sensing Systems}

A particular advantage of our design is its ability to measure respiration in the immediate vicinity of a single cell in the micro-chamber, eliminating the uncertainty of relative positioning in other methods such as SECM $[42,116]$. These instruments are bulky with inconsistent performance and high cost compared to the micro-chamber system described herein. Most SECM-based techniques involve movement of the sensor tip between the cell and bulk solution. 
The scanning system needs a precise positioner and motors to achieve an accurate control of the tip's position, and multiple measurement sites are needed.

More recently, the most widely used instrument for assessment of cellular metabolism is the Extracellular Flux Analyzer (Agilent Technologies, Santa Clara, CA), which is capable of monitoring rates of glycolysis (via extracellular acidification rate) and oxygen consumption in cell populations by fluorescence techniques in a multi-well format [44-45]. However, this analyzer is extremely expensive, requires separate assays to measure glycolysis and oxygen consumption, and generally requires hundreds to thousands of cells per assay. In this work, we describe an integrated metabolic multi-sensor capable of monitoring single embryo oxygen consumption, glucose uptake, and lactate production in real-time using amperometric methods, providing a simple and inexpensive method of monitoring single embryo metabolism during development in a small volume of medium. 


\section{Chapter 6: Design of A Multi-Sensor Platform for Integrating Extracellular Acidification Rate with Multi-Metabolite Flux Measurement for Small Biological Samples}

\subsection{Background of the System}

Rates of cellular oxygen consumption (OCR) and extracellular acidification (ECAR) are widely used proxies for mitochondrial oxidative phosphorylation (OXPHOS) and glycolytic rate in cell metabolism studies. However, ECAR can result from both oxidative metabolism (carbonic acid formation) and glycolysis (lactate release), potentially leading to erroneous conclusions about metabolic substrate utilization. Co-measurement of extracellular glucose and lactate flux along with OCR and ECAR can improve the accuracy and provide better insight into cellular metabolic processes but is currently not feasible with any commercially available instrumentation. Herein, we present a miniaturized multi-sensor platform capable of real-time monitoring of OCR and ECAR along with extracellular lactate and glucose flux for small biological samples such as single equine embryos. This multiplexed approach enables validation of ECAR resulting from OXPHOS versus glycolysis, and expression of metabolic flux ratios that provide further insight into cellular substrate utilization. We demonstrate expected shifts in embryo metabolism during development and in response to OXPHOS inhibition as a model system for monitoring metabolic plasticity in very small biological samples. Furthermore, we also present a preliminary interference analysis of the multi-sensor platform to allow better understanding of sensor interference in the proposed multi-sensor platform. The capability of the platform is illustrated with measurements multimetabolites of single-cell equine embryos for assisted reproduction technologies. However, this platform has a wide potential utility for analyzing small biological samples such as single cells and tumor biopsies for immunology and cancer research applications. The details of this chapter are largely based on a submitted journal paper, "Design of A Multi-Sensor Platform for Integrating Extracellular Acidification Rate with Multi-Metabolite Flux Measurement for Small Biological Samples [117]". 


\subsection{Materials and Methods}

\subsubsection{Materials and Reagents}

pH meter was purchased from (Hanna instruments, Woonsocket RI, USA). Indium Tin Oxide (ITO) coated glass substrates was purchased from Sigma Aldrich (St. Louis, Missouri). The rest of the materials used are similar to what was included in Section 5.2.1 in Chapter 5.

\subsubsection{Electrodes and Chamber Designs and Manufacturing}

The multi-sensor chip has an Indium Tin Oxide (ITO) electrode for $\mathrm{pH}$ measurement and gold (Au) electrodes for measuring the rest of the target metabolites. The masks were designed using AutoCAD® software (Autodesk, Inc.) which was used for generating electrodes patterns (Figs.

6.1A and 6.1B). The sensor chip was fabricated using an ITO coated glass substrate of $24.5 \mathrm{~mm}$ $\mathrm{x} 24.5 \mathrm{~mm}$ as the base to form the ITO electrode pattern through standard photolithography and etching. The Au electrodes are formed afterwards through standard photolithography, thermal evaporation, and lift-off techniques. The three-electrode configuration with working electrode (WE), quasi reference electrode (RE), and counter electrode (CE) was used for allamperometric sensor configurations. A Two-electrodes configuration was used for $\mathrm{pH}$ sensing (Fig. 6.1C). Details of the mask design, photolithography, metal evaporation, and lift-off are included in Section 5.2.2. ITO etching is described in Section 6.2.3. A $12 \mathrm{~mm}$ diameter and $3 \mathrm{~mm}$ deep micro-chamber containing all three sensors was made using SU8 with details provided in Section

\subsection{2.}

\subsubsection{ITO Etching}

The ITO pattern for the $\mathrm{pH}$ sensor was created on an ITO coated glass substrate using the photolithography technique and etching. ITO etching was done using ITO TE-100 etchant after heating at temperature of $60{ }^{\circ} \mathrm{C}$ on the hot plate for 8-10 min. The chip was rinsed with DI water and dried with $\mathrm{N}_{2}$ after the etching was done. The ITO electrode has a total area of $8 \mathrm{~mm}^{2}$. 

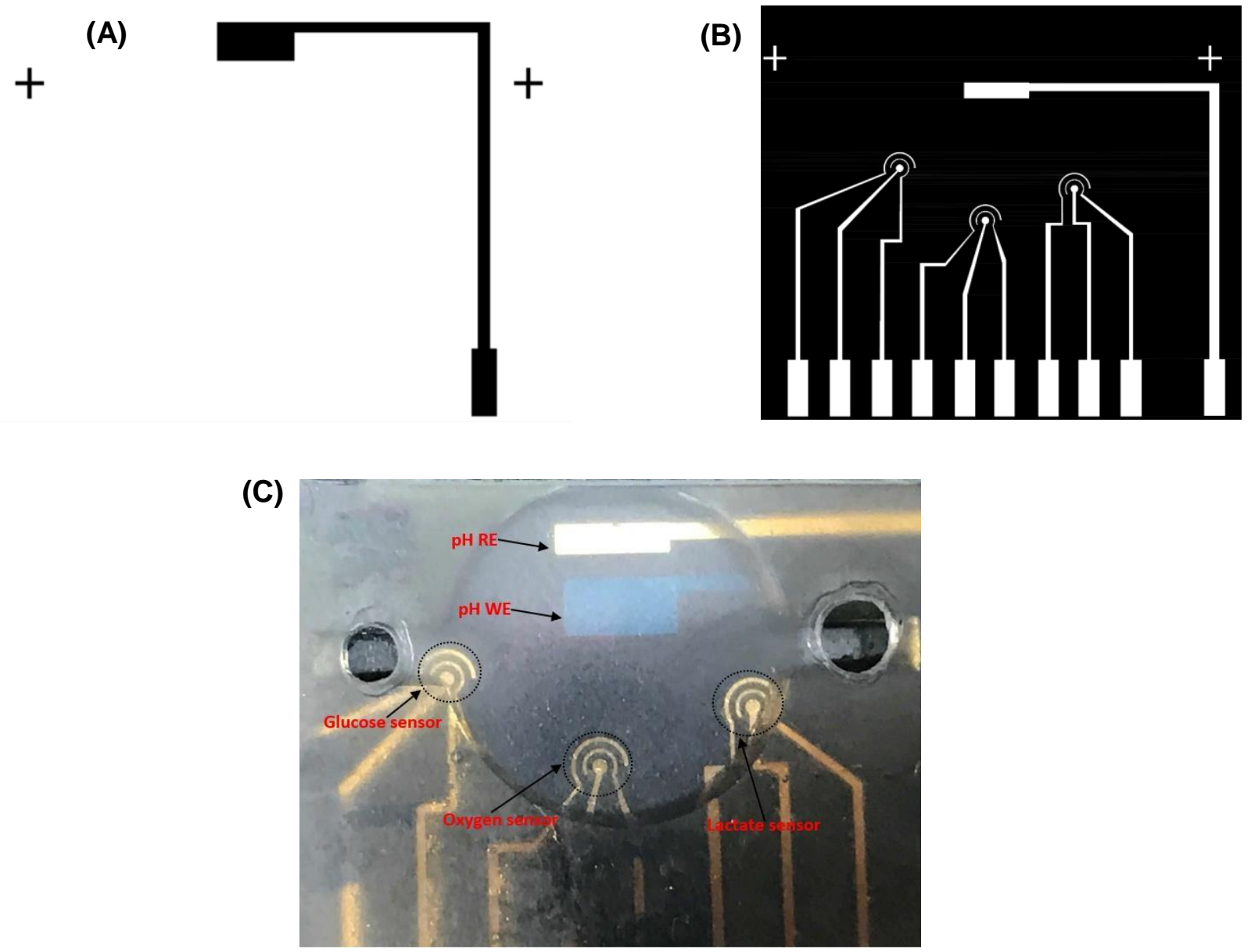

Fig. 6.1. (A) Mask design for ITO electrode pattern. (B) Mask design for gold electrodes patterns. (C) Complete design with $3 \mathrm{~mm}$ thick SU8 micro-chamber covered by a glass lid.

\subsubsection{Sensors Surfaces Modification}

The techniques for surface modifications for the oxygen, glucose and lactate sensors are the same as those described in Chapter 5.

\subsubsection{Sensor Activation Voltages}

The activation voltage for each sensor was determined through a set of CV experiments. The activation voltages for $\mathrm{DO}$, glucose, and lactate, were found to be $-0.6 \mathrm{~V}, 0.4 \mathrm{~V}$, and $0.4 \mathrm{~V}$, respectively (Section 5.2.6). 


\subsubsection{Sensors Calibration}

\subsubsection{Calibration for Oxygen, Glucose and Lactate Sensors}

The methods used for calibrating the oxygen, glucose and lactate sensors are the same as those described in Chapter $\mathbf{5}$. The calibration curves in Chapter $\mathbf{5}$ assume a fixed $\mathrm{pH}$ value of the buffer at the beginning of experiments. However, during cell basal respiration and glycolysis, the $\mathrm{pH}$ level decreases which, in turn, affect the response of both glucose and lactate enzymes [118-119]. To understand the potential measurement errors for glucose and lactate due to changing buffer $\mathrm{pH}$ during experiments, we measured the effect of $\mathrm{pH}$ change on lactate and glucose sensors responses and added $\mathrm{pH}$ as a calibration variable.

\subsubsection{Calibration for $\mathrm{pH}$ Sensor}

To determine the calibration curve and linearity of $\mathrm{pH}$ sensor, solutions of different $\mathrm{pH}$ values were made by diluting sodium hydroxide $\mathrm{NaOH}$ or hydrogen chloride $\mathrm{HCL}$ in $2 \mathrm{mM}$ potassium chloride $(\mathrm{KCl})$ using concentration vs volume equation $\mathrm{C}_{1} \mathrm{~V}_{1}=\mathrm{C}_{2} \mathrm{~V}_{2}$. The $\mathrm{KCl}$ solution was used for dilution to prepare solutions with different $\mathrm{pH}$ values with full ionic strength [120]. The value of $\mathrm{pH}$ was validated using a commercial pH meter (Hanna instruments, Woonsocket RI, USA).

The change in voltage corresponding to $\mathrm{pH}$ change were measured and recorded using a circuit based on the INA333 instrumentation amplifier (Analog Devices, Norwood, MA). The pH sensor responses were plotted against the $\mathrm{pH}$ level (relative percentage of hydrogen ions) to determine sensor sensitivity, linearity, and dynamic range.

\subsubsection{Measurement Setup}

\subsubsection{Oxygen, Glucose, Lactate and pH Measurements}

The multi-sensor chip embedded in the micro-chamber described in Section 6.2 was used. The temperature of the medium was maintained at $38.5^{\circ} \mathrm{C}$ by placing the device on a stage warmer for a stereomicroscope. The respective activation voltages were applied during all the amperometric experiments for DO, glucose, and lactate. 
The electrodes were rinsed using DI water and electrochemically pulse cleaned for $1 \mathrm{~min}$ before and after each test. Sterilized DI water was used for pulse cleaning to avoid any toxic effect on cells from any other cleaning chemicals. The amplitude of pulses used for cleaning was $1.2 \mathrm{~V}$ (from $-0.6 \mathrm{~V}$ to $0.6 \mathrm{~V}$ ) and the pulse duration was $2 \mathrm{~ms}$.

Two separate micro-chambers were used for measurement; one chamber was used for measuring oxygen and $\mathrm{pH}$ and the other chamber was used for measuring glucose and lactate. To reliably measure the oxygen consumption rate (OCR) of a given embryo, the embryo was put in the micro-chamber with the oxygen and $\mathrm{pH}$ sensors activated for a period of time long enough for the OCR signal to reach a stable reading, followed by a reading of the $\mathrm{pH}$ value in the microchamber. The micro-chamber contains $250 \mu \mathrm{L}$ G-MOPS medium (respiration buffer). Once the stable OCR and $\mathrm{pH}$ readings were obtained, $2.5 \mu \mathrm{L}$ of the G-MOPS medium each was extracted from the micro-chamber and moved to the second micro-chamber with the glucose and lactate sensors activated for glucose and lactate measurements. Each extracted $2.5 \mu \mathrm{L}$ of the G-MOPS medium covered the entire area of either the glucose or the lactate sensor site only in the second micro-chamber. Once the measurements were made, the extracted G-MOPS medium droplets were removed from the glucose and lactate sensor sites and the sensor sites were then cleaned with DI-water and dried and ready for the next round of measurement. Due to the required enzymatic surface modifications for the glucose and lactate sensors and the proximity of the sensors inside the micro-chamber, there are two reasons for separating the micro-chambers. First of all, enzymatic reactions on both the glucose sensor and the lactate sensor produce corresponding $\mathrm{H}_{2} \mathrm{O}_{2}$ which, in turn, is electrochemically detected by the sensor. The proximity of the sensors within the micro-chamber may create interference due to diffusion of $\mathrm{H}_{2} \mathrm{O}_{2}$ between the sensor sites. Even though it was not clear such interference could have impacted the accuracy of the glucose and lactate measurement, we were cautious to avoid the possibility of such interference during the experiments. 
Further studies of interference and its preliminary findings are discussed later in this chapter. Secondly, the toxicity of enzymes used for electrode surface modification for the glucose and the lactate sensors is still under investigation. To avoid potential toxicity affecting embryos, embryos under measurements were not put in the same micro-chamber to avoid direct contact with enzyme layers.

Based on morphology, embryos separated into four groups: Group 1, small or early blastocysts; 2, blastocysts with a defined blastocoel and thinning trophoblast layer; 3 , expanded blastocysts with expansion of the blastocoel, increased size of the embryo or extrusion of the cellular mass through the encircling zona pellucida, and 4) non-viable embryo, embryos that stopped developing in culture and appeared to be degenerating, considered a negative control.

Equine embryos from different groups (1-4) of different stages were tested during these experiments. Group 1 includes small or early blastocysts (SB/EB); group 2 includes blastocysts (B); group 3 includes expanded blastocysts (XB); and group 4 includes non-viable (dead) embryos. Oxygen consumption and $\mathrm{pH}$ were measured in two cycles; in each cycle oxygen was measured for 10-12 min and then $\mathrm{pH}$ was measured for $2 \mathrm{~min}$, then a droplet of $2.5 \mu \mathrm{L}$ of GMOPS medium was taken and placed on top of the glucose sensor and reading was measured for $2 \mathrm{~min}$, then a second droplet of $2.5 \mu \mathrm{L}$ of G-MOPS medium was taken and placed on top of the lactate sensor and reading was measured for 2 min. Oligomycin $(1 \mu \mathrm{M})$ was used as an ATP synthase inhibitor and all cycles were repeated after adding it. FCCP titration $(1 \mu \mathrm{M})$ was used to reveal the maximal cellular respiratory capacity and all cycles were repeated to measure its effect on all rates of oxygen, glucose, lactate and $\mathrm{pH}$.

The oxygen consumption, glucose consumption, and lactate production rates were calculated based on a model described in Section 5.2.9.

The $\mathrm{pH}$ change was measured by converting the change in voltage during cell respiration in a defined buffer volume to $\mathrm{pH}$ level change using the calibration curve and the following equation: 
$\Delta p H=\frac{\Delta V}{\text { sensitivity }\left(\frac{m V}{p H}\right)}$

where $\Delta \mathrm{V}$ is the measured change in voltage, and the sensitivity is the slope of $\mathrm{pH}$ sensor calibration curve.

\subsubsection{Sensor Interference Tests}

Since glucose and lactate are both measured via electrochemical detection of the enzymatic release of $\mathrm{H}_{2} \mathrm{O}_{2}$ under the same activation potential, interference between the glucose and the lactate sensors may exist if the glucose and lactate working electrodes are close to each other. The enzymatically generated $\mathrm{H}_{2} \mathrm{O}_{2}$ at one sensor site (e.g. the lactate sensor) can be diffused to the nearby glucose sensor over time that also relies on $\mathrm{H}_{2} \mathrm{O}_{2}$ of its own to generate redox current. To reduce the possibility of interference, one can increase the separation distance between the working electrodes. However, this will make the micro-chamber too big to hold the desired amount of buffer volume of G-MOPS for the required sensitivity of single-cell respiration measurement. Furthermore, the degree of interference also depends on the intrinsic quantities of associated target analytes in the buffers. Previously, we illustrated the effect of interference between glucose and lactate sensors within a single micro-chamber. The enzymatically generated $\mathrm{H}_{2} \mathrm{O}_{2}$ at the lactate sensor moves towards the glucose sensor through diffusion. The detectable amount of diffused $\mathrm{H}_{2} \mathrm{O}_{2}$ took about 40 minutes to reach the glucose sensor site. However, the enzymatically generated $\mathrm{H}_{2} \mathrm{O}_{2}$ diffused from the glucose sensor has much less impact on the lactate sensor reading through diffusion. This is because the G-MOPS buffer has a much higher concentration of lactate than that of glucose. The G-MOPS respiration buffer contains $0.5 \mathrm{mM}$ glucose and 5.3 $\mathrm{mM}$ lactate. The interference between the glucose and lactate sensors puts a limitation on the measurement time period in order to avoid measurement inaccuracy. 
To further understand inter-sensor interference and their relationships with sensor distance and measured target analyte concentration in a given buffer, a different multi-sensor configuration was used with smaller sensor distances for interference experiments (Fig. 6.2).

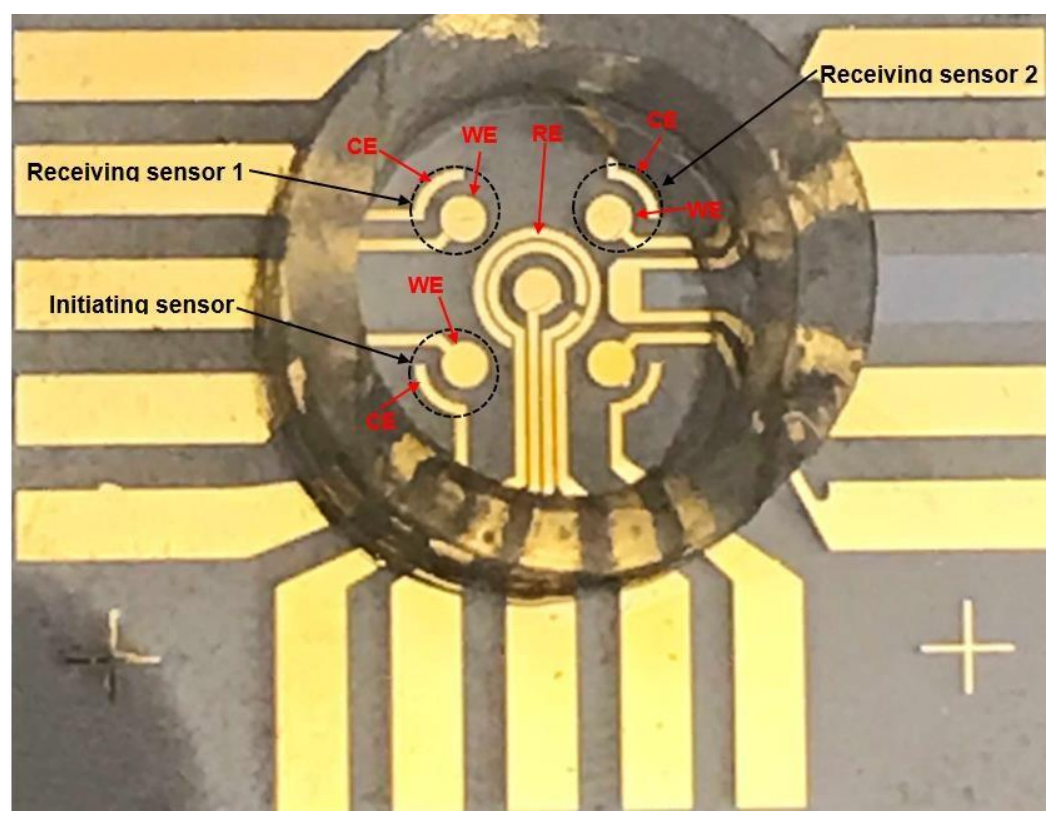

Fig. 6.2. The sensor design used for interference test.

The interference tests use three of the four corner sensors, one sensor is the initiating sensor and the other two sensors are receiving sensors with differing distances to the initiating sensor. The initiating sensor produces $\mathrm{H}_{2} \mathrm{O}_{2}$ from its enzyme layer from one of the analytes (glucose or lactate); the receiving sensors are turned on to electrochemically detect traces of $\mathrm{H}_{2} \mathrm{O}_{2}$ diffused from the initiating sensors four different concentrations of glucose and lactate $(0.1 \mathrm{mM}, 0.5 \mathrm{mM}$, $1 \mathrm{mM}$ and $2 \mathrm{mM}$ ) were used for the interference tests.

Three participating sensors (one initiating and two receiving sensors) were monitored by three Potentiostat channels simultaneously to measure their responses over time. Each concentration was measured for 2 hours. In one test the effects of lactate titration from the initiating sensor were measured on two glucose receiving sensors at different distances of 3.2 and $4.6 \mathrm{~mm}$ from the lactate initiating sensor; in another test, the effects of glucose titration from 
the initiating sensor were measured on two lactate receiving sensors at different distances of 3.2 and $4.6 \mathrm{~mm}$ from the glucose initiating sensor.

\subsection{Results and Discussion}

\subsubsection{Sensors Calibration}

\subsubsection{Oxygen, Glucose and Lactate Sensors Calibration Results}

The processes of calibrating oxygen, glucose, and lactate sensors with a fixed $\mathrm{pH}$ value and the calibration results are already included in Chapter 5. However, as discussed in Section 6.2.6.1, calibrations for glucose and lactate sensors should also include the impact of changing $\mathrm{pH}$ during the experiments. The new calibration surfaces with $\mathrm{pH}$ as one of the variables for glucose and lactate are presented in Figs. (6.3A and 6.3B). The effect of changing pHwill make the glucose and lactate readings based on the calibration curve with a fixed $\mathrm{pH}$ value deviate from their true readings.

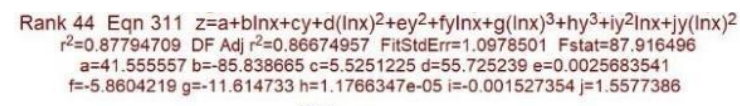

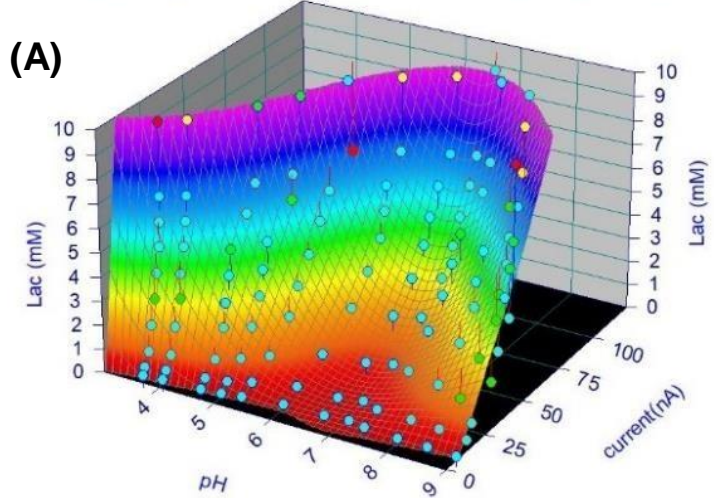

Fig. 6.3. (A) Lactate sensor 3D calibration curve. (B) Glucose sensor 3D calibration curve.

\subsubsection{2 pH Sensor Calibration}

Fig. 6.4 shows the $\mathrm{pH}$ sensor calibration results, the sensor has a wide dynamic range from $\mathrm{pH} 1$ to $\mathrm{pH} 14$, a good linearity of 0.99 and a sensitivity of $-54.74 \mathrm{mV} / \mathrm{pH}$ that is close to what was reported in previous studies [47]. 


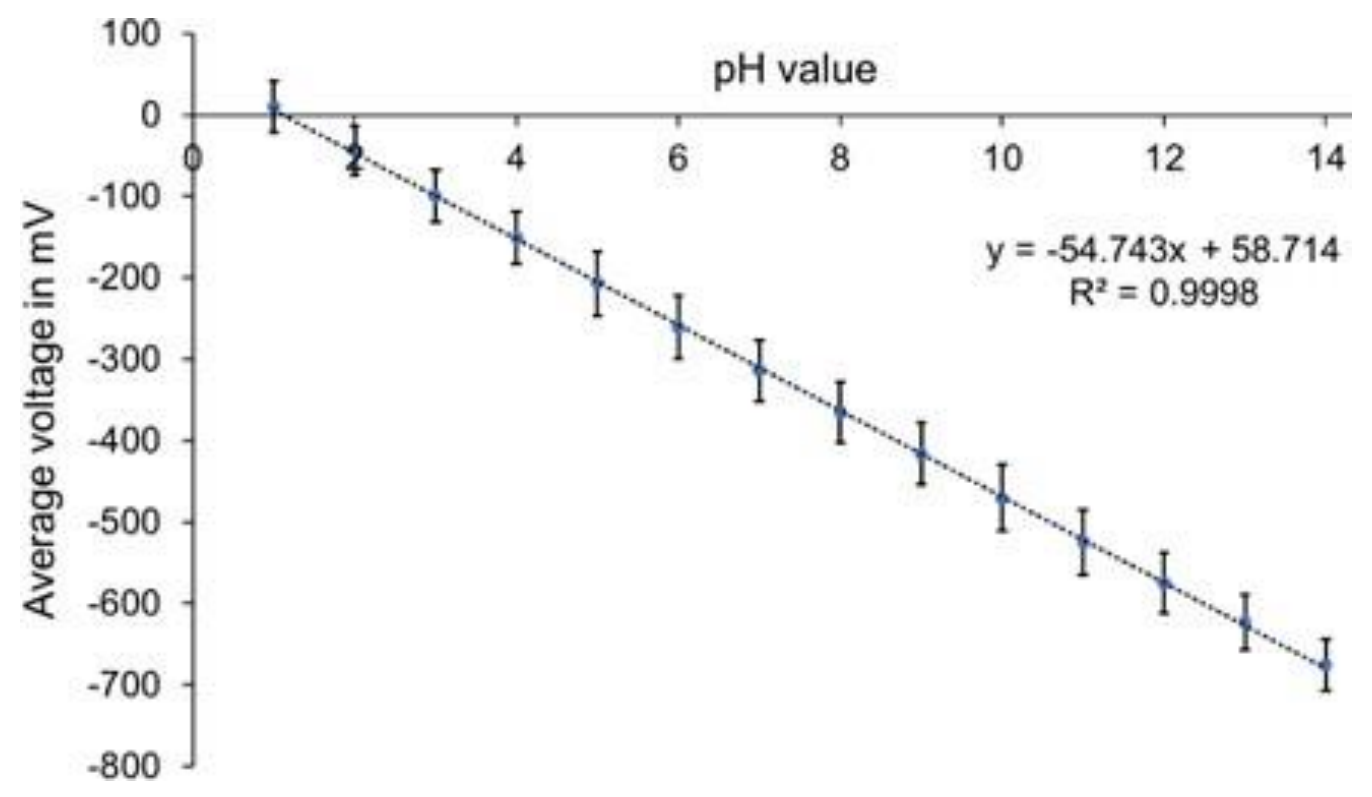

Fig. 6.4. pH sensor calibration curve (Error bars in each curve are standard deviations (SD) between 6 data points).

\subsubsection{Characterization of Equine Embryo Energy Metabolism}

\subsubsection{Evaluation of Equine Embryo Metabolism}

To investigate the metabolic characteristics of embryos, four groups of embryos were used for metabolism studies. Glucose and oxygen consumption rates (GCR and OCR) and lactate production rate (LPR) are expressed as fmol per embryo per second \pm standard error (SE). and ECAR is presented in $\mathrm{mpH}$ per embryo per minute $\pm \mathrm{SE}$. The patterns of oxygen, glucose, lactate and ECAR of groups 1 to 4 was analyzed by one-way analysis of variance (ANOVA) between the groups. Differences between means were examined using Tukey HSD (pairwise comparison among stages). Paired t-test was used to test the differences between different conditions (basal, oligo, and FCCP) within the same group. Group 4 (non-viable embryos) showed minimum basal respiration and minimum response to oligo, and FCCP compared to groups 1 to 3 (Fig.6.5) 


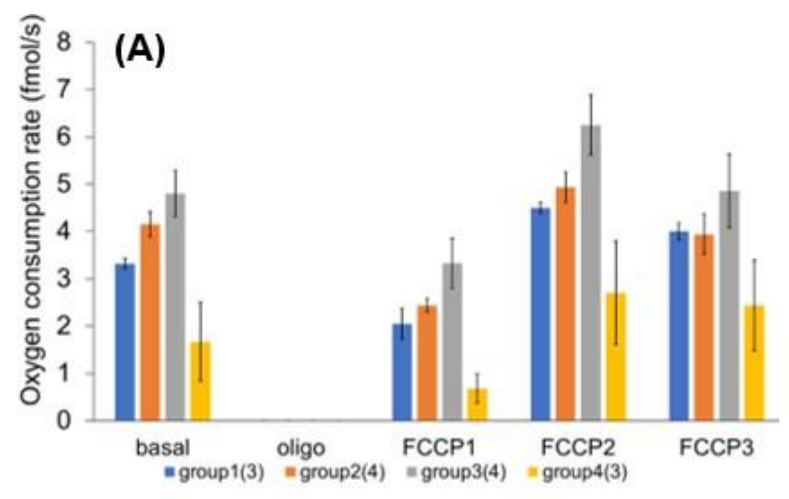

(B)
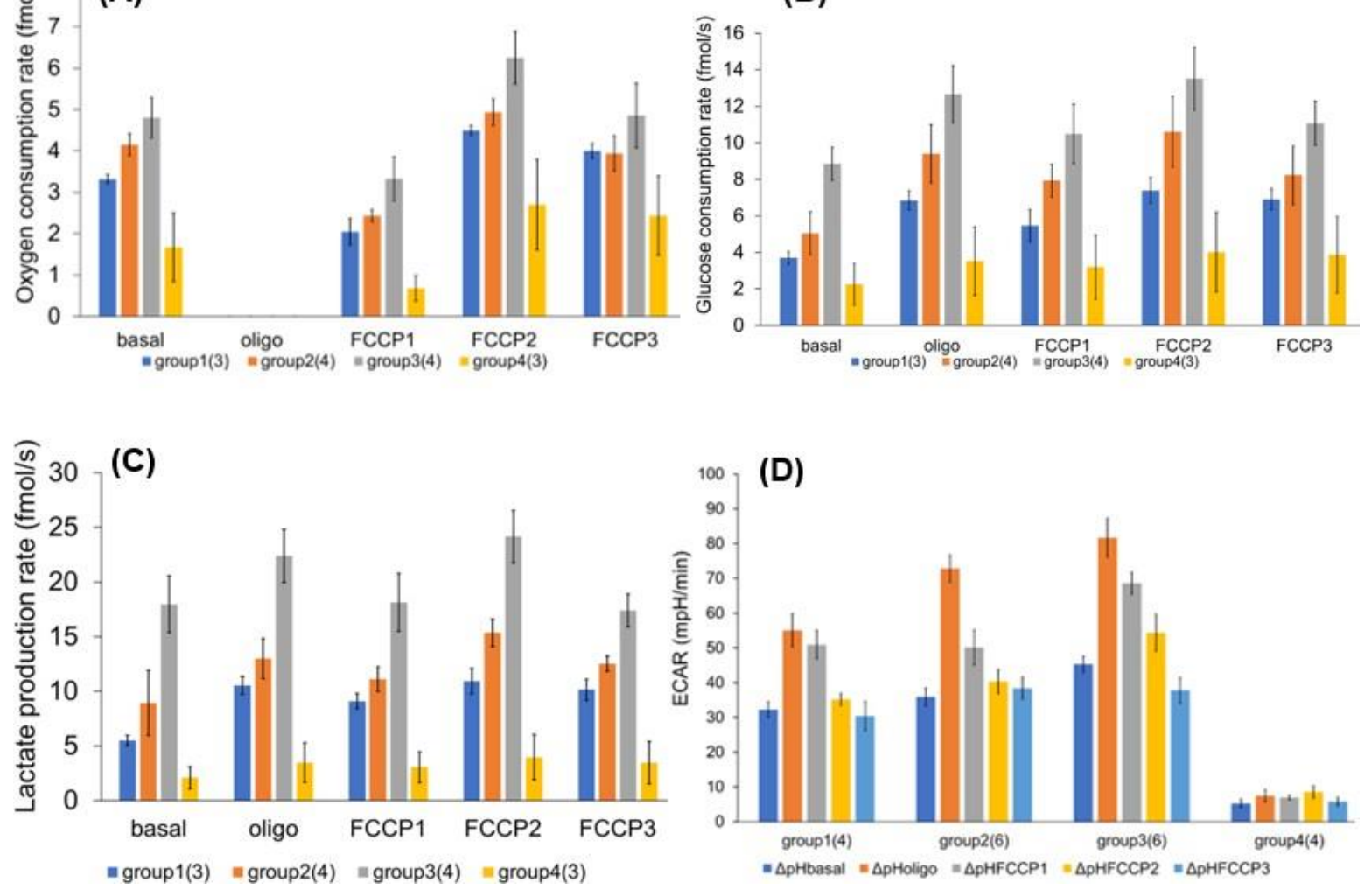

Fig. 6.5. Average rates for equine embryos before and after adding $1 \mu \mathrm{M}$ oligomycin and $1 \mu \mathrm{M}$ FCCP titration (mean \pm SE): (A) OCR (B) GCR (C) LPR (D) ECAR.

All parameters of basal metabolic flux (OCR, GCR, LPR, and ECAR) increased ( $P \leq 0.05)$ from $B$ and $X B$ stages in viable equine embryos (Figs. 6.6A-6.6D). (See Tables 6.1 to 6.3 for pairwise comparison). 

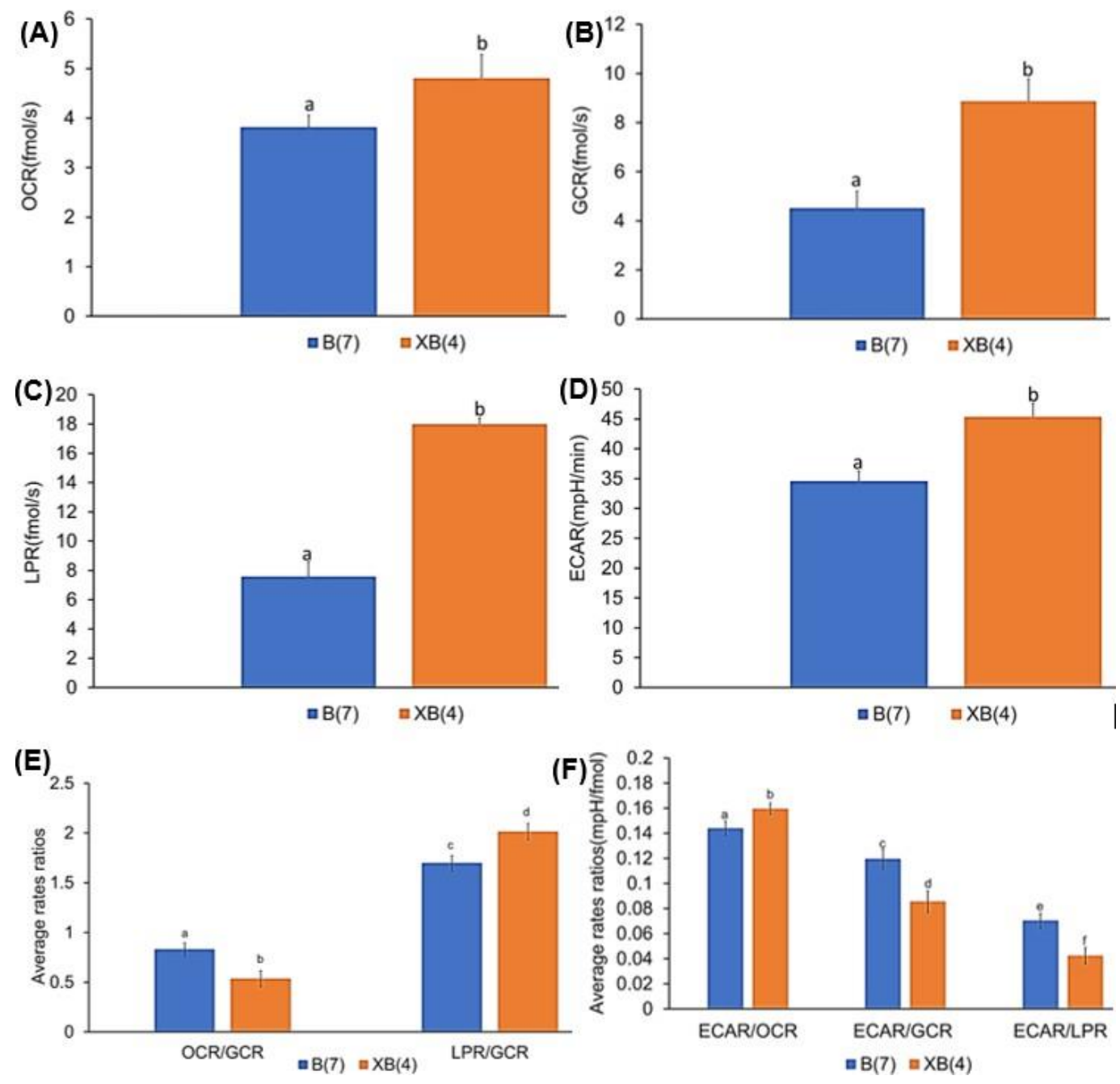

Fig.6.6. Rates for $B$ and $X B$ equine embryos: (A) basal OCR (mean \pm standard of error (SE)).

(B) basal GCR (mean \pm SE). (C) basal LPR (mean \pm SE). (D) basal ECAR (mean $\pm S E$ ). (E) basal OCR/GCR and LPR/GCR (mean \pm SE). (E) basal ECAR/OCR, ECAR/GCR and ECAR/LPR (mean \pm SE).

Table 6.1: basal OCR, GCR, ECAR and LPR of in vitro produced equine embryos. One-way ANOVA for all data is $(P<0.001)$. a, b, c, d within columns: Tukey HSD (pairwise comparison), values with different superscripts are significantly different $(P \leq 0.05)$.

\begin{tabular}{|l|l|l|l|l|}
\hline \multicolumn{1}{|c|}{ group } & \multicolumn{1}{|c|}{ OCR (fmol/s) \pm} & \multicolumn{1}{|c|}{ GCR $(\mathrm{fmol} / \mathrm{s}) \pm$} & \multicolumn{1}{c|}{$\mathrm{LPR}((\mathrm{fmol} / \mathrm{s}) \pm$} & $\begin{array}{l}\mathrm{ECAR}(\mathrm{mpH} / \mathrm{min} \pm \\
\mathrm{SE})\end{array}$ \\
\hline $\begin{array}{l}\text { Group 1 } \\
(3)\end{array}$ & $3.315 \pm 0.1^{\mathrm{a}}$ & $3.7 \pm 0.35^{\mathrm{a}}$ & $5.52 \pm 0.46^{\mathrm{a}}$ & $32.3 \pm 2.2^{\mathrm{a}}$ \\
\hline $\begin{array}{l}\text { Group 2 } \\
(5)\end{array}$ & $4.15 \pm 0.26^{\mathrm{ab}}$ & $5.04 \pm 1.1^{\mathrm{ab}}$ & $8.93 \pm 2.9^{\mathrm{ab}}$ & $35.9 \pm 2.6^{\mathrm{ab}}$ \\
\hline $\begin{array}{l}\text { Group 3 } \\
\text { (6) }\end{array}$ & $4.8 \pm 0.48^{\mathrm{c}}$ & $8.86 \pm 0.9^{\mathrm{c}}$ & $17.97 \pm 2.6^{\mathrm{c}}$ & $45.32 \pm 2.2^{\mathrm{c}}$ \\
\hline $\begin{array}{l}\text { Group } \\
4(4)\end{array}$ & $1.6 \pm 0.82^{\mathrm{d}}$ & $2.26 \pm 1.14^{\mathrm{d}}$ & $2.12 \pm 1.01^{\mathrm{d}}$ & $5.27 \pm 1.6^{\mathrm{d}}$ \\
\hline
\end{tabular}


Table 6.2: OCR, GCR, ECAR and LPR of in vitro produced equine embryos after adding oligomycin. One-way ANOVA for all data is $(\mathrm{P}<0.001)$. a, b, c, d within columns: Tukey HSD (pairwise comparison), values with different superscripts are significantly different $(P \leq 0.05)$.

\begin{tabular}{|l|l|l|l|l|}
\hline \multicolumn{1}{|c|}{ group } & \multicolumn{1}{|c|}{$\begin{array}{c}\text { OCR (fmol/s) } \pm \\
\text { SE }\end{array}$} & $\begin{array}{c}\text { GCR (fmol/s) } \pm \\
\text { SE }\end{array}$ & \multicolumn{1}{|c|}{$\begin{array}{c}\text { LPR (fmol/s) } \pm \\
\text { SE) }\end{array}$} & \multicolumn{1}{|c|}{$\begin{array}{c}\text { ECAR }(\mathrm{mpH} / \mathrm{min} \pm \\
\text { SE) }\end{array}$} \\
\hline $\begin{array}{l}\text { Group 1 } \\
(3)\end{array}$ & $0.0003 \pm 0.00012^{\mathrm{a}}$ & $6.85 \pm 0.85^{\mathrm{a}}$ & $10.55 \pm 0.81^{\mathrm{a}}$ & $55.07 \pm 4.7^{\mathrm{a}}$ \\
\hline $\begin{array}{l}\text { Group 2 } \\
(5)\end{array}$ & $\begin{array}{l}0.00129 \pm 0.0002 \\
2^{\mathrm{ab}}\end{array}$ & $9.4 \pm 1.6^{\mathrm{ab}}$ & $13 \pm 1.8^{\mathrm{ab}}$ & $72.79 \pm 3.8^{\mathrm{ab}}$ \\
\hline $\begin{array}{l}\text { Group 3 } \\
(6)\end{array}$ & $0.0023 \pm 0.0008^{\mathrm{c}}$ & $12.65 \pm 1.55^{\mathrm{c}}$ & $22.4 \pm 2.4^{\mathrm{c}}$ & $81.67 \pm 5.63^{\mathrm{c}}$ \\
\hline $\begin{array}{l}\text { Group } \\
4(4)\end{array}$ & $\begin{array}{l}0.00151 \pm 0.0 .000 \\
90^{\mathrm{d}}\end{array}$ & $3.53 \pm 1.88^{\mathrm{d}}$ & $3.49 \pm 1.8^{\mathrm{d}}$ & $57.45 \pm 1.74^{\mathrm{d}}$ \\
\hline
\end{tabular}

Table 6.3: Oxygen consumption, glucose uptake, ECAR and lactate production of in vitro produced equine embryos at max respiration (FCCP2). One-way ANOVA for all data is $(\mathrm{P}<0.001)$. a, b, c, d within columns: Tukey HSD (pairwise comparison), values with different superscripts are significantly different $(P \leq 0.05)$.

\begin{tabular}{|l|l|l|l|l|}
\hline \multicolumn{1}{|c|}{ group } & OCR $(\mathrm{fmol} / \mathrm{s}) \pm \mathrm{SE}$ & $\begin{array}{l}\text { GCR }(\mathrm{fmol} / \mathrm{s}) \pm \\
\text { SE }\end{array}$ & LPR $(\mathrm{fmol} / \mathrm{s}) \pm \mathrm{SE}$ & $\begin{array}{c}\text { ECAR } \\
(\mathrm{mpH} / \mathrm{min} \pm \\
\text { SE) }\end{array}$ \\
\hline $\begin{array}{l}\text { Group 1 } \\
(3)\end{array}$ & $4.5 \pm 0.115^{\mathrm{a}}$ & $7.4 \pm 0.7^{\mathrm{a}}$ & $10.95 \pm 1.1^{\mathrm{a}}$ & $35.19 \pm 1.7^{\mathrm{a}}$ \\
\hline $\begin{array}{l}\text { Group 2 } \\
(5)\end{array}$ & $4.9 \pm 0.31^{\mathrm{ab}}$ & $10.6 \pm 1.9^{\mathrm{ab}}$ & $15.37 \pm 1.25^{\mathrm{ab}}$ & $40.35 \pm 3.5^{\mathrm{ab}}$ \\
\hline $\begin{array}{l}\text { Group 3 } \\
(6)\end{array}$ & $6.25 \pm 0.63^{\mathrm{c}}$ & $13.5 \pm 1.7^{\mathrm{c}}$ & $24.16 \pm 2.4^{\mathrm{c}}$ & $54.37 \pm 5.3^{\mathrm{c}}$ \\
\hline Group 4(4) & $2.7 \pm 1.09^{\mathrm{d}}$ & $4.01 \pm 2.18^{\mathrm{d}}$ & $3.9 \pm 2.07^{\mathrm{d}}$ & $8.5 \pm 1.7^{\mathrm{d}}$ \\
\hline
\end{tabular}

The observed increases in basal OCR, GCR, LPR, from B to XB are consistent with our previous results on bovine embryos in Chapter 5 and other previous studies [98] and generally reflect the increase in embryo energy and nutrient demands as cell numbers increase [103, 104].

The observed increase of ECAR from $B$ to $X B$ stage suggests a greater reliance upon anaerobic glycolysis to meet energy demands during blastocyst expansion, consistent with the observed increase in LPR. This interpretation is further supported by a decline in the OCR/GCR ratio (indicating a lesser contribution of OXPHOS to total glucose utilization) and higher LPR/GCR ratio (suggesting a greater utilization of glucose for lactate production) from B to XB (Fig. 6.6E). 
Interestingly, integrating ECAR into these metabolite flux analyses revealed insights that highlight the greater complexity of metabolic substrate utilization in developing embryos (Fig. 6.6F). As expected, the ECAR/OCR increased from $B$ to $X B$, which is classically interpreted a greater contribution of anaerobic glycolysis relative to OXPHOS. However, both ECAR/GCR and ECAR/LPR declined from $B$ to $X B$, indicating a greater proportion of glucose consumed by expanded blastocysts is not contributing to ECAR. Interpretation of this result requires a more comprehensive view of nutrient metabolism and the potential courses of OCR and ECAR in developing embryos, which is summarized in Fig. 6.7.

Importantly, a developing embryo uses glucose both for ATP production and biosynthetic processes that are essential for cell growth and proliferation [124]. These pathways (in particular, the pentose phosphate and one-carbon metabolism pathways) result in much less $\mathrm{CO}_{2}$ production or and net $\mathrm{H}+$ released from glucose consumed because more of the carbons of glucose (and other nutrients) stay inside the cell to build cellular components such as DNA, proteins, and lipids, as well as mediate epigenetic programming (methylation) of developing genes [125, 126]. Therefore, the lower ECAR relative to glucose utilization in expanding blastocysts likely reflects an increasing proportion of glucose uptake contributing to biosynthetic processes rather than glycolytic ATP production. Moreover, shifts in the uptake and utilization of other metabolic substrates (e.g., pyruvate and fatty acids) can decrease the net cellular release of $\mathrm{H}+$ and alter the stoichiometry of $\mathrm{OCR}$ and $\mathrm{CO}_{2}$ production, further complicating interpretation of changes in ECAR and OCR. Similar metabolic shifts occur in proliferating cancer cells [127, 128], and are being increasingly recognized as an important consideration in the study of cellular glucose utilization and bioenergetics in this context [129]. Therefore, integrating simultaneous OCR and ECAR measurements with real-time metabolite flux analyses can significantly improve the interpretive value of isolated measurements performed in these complex metabolic systems. The multi-sensor approach described herein provides a miniaturized platform for performing these 
integrative analyses in very small primary samples, which could be complimented by additional metabolite sensors or stable isotope-tracer technologies to further elucidate metabolic aspects of cellular development and proliferation in health and disease.

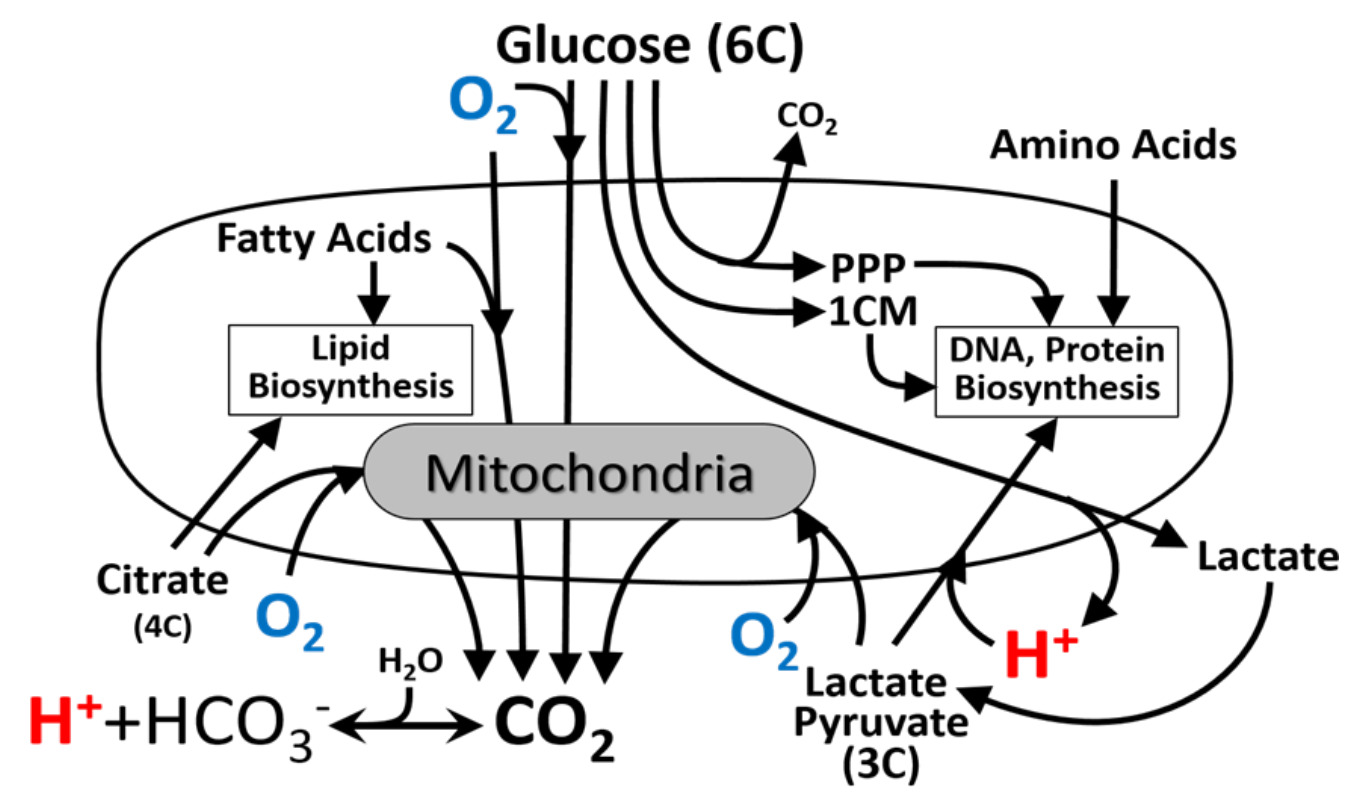

Fig. 6.7. Sources of OCR and ECAR and metabolite flux in developing embryos. Oxygen $\left(\mathrm{O}_{2}\right)$ is consumed by respiring mitochondria in cells to support oxidative metabolism of multiple substrates. Hydrogen ions $\left(\mathrm{H}_{+}\right)$are exported from cells with lactate but can also be generated from $\mathrm{CO}_{2}$ in the aqueous G-MOPS buffer or co-transported into cells along with pyruvate or lactate. The six carbons of glucose can be released as $\mathrm{CO}_{2}$ during oxidative metabolism, as two lactate molecules following glycolysis, or utilized in the biosynthetic pentose phosphate pathway (PPP) and one-carbon metabolism (1CM) pathway.

\subsubsection{Effect of Oligomycin and FCCP Titration on Embryo Metabolism}

To further evaluate the effect of metabolic shifts on individual and integrated metabolite flux measurements in this system, we tested the effect of blocking mitochondrial ATP synthesis (with oligomycin) and chemical uncoupling of oxidative metabolism from respiration (with FCCP) on developing embryos. Figs. 6.8A-6.8D illustrate the combined averages of OCR, GCR, LPR and ECAR in embryos at all developmental stages under basal conditions followed by the subsequent titration of oligomycin and FCCP. As expected, oligomycin stopped OCR and forced embryos to rely more heavily on glucose metabolism indicated by higher GCR, LPR and ECAR, consistent with our previous findings in Chapter 5. FCCP was then carefully titrated in $1 \mu \mathrm{M}$ steps to pinpoint 
the maximal "non-coupled" rate of embryo OCR (at $\sim 2 \mu \mathrm{M})$, after which an inhibitory effect becomes evident (Fig. 6.5). This acceleration of uncoupled OCR by FCCP further increased GCR, and to a lesser extent LPR, consistent with a re-activation of glucose oxidation by mitochondria, but continued reliance on glycolytic ATP production due to inhibition of mitochondrial ATP production. However, this uncoupling of mitochondrial respiration with FCCP strongly decreased ECAR, suggesting a significant shift in embryo redox state under these conditions. FCCP collapses the mitochondrial membrane potential, leading to acidification of the mitochondrial matrix until it equilibrates with the cell $\mathrm{H}+$ content. This creates a redox imbalance in the cytosol that favors cellular retention of $\mathrm{H}+$ to maintain reducing power, perhaps leading to lower net release of $\mathrm{H}+$ observed as lower ECAR. Mitochondrial substrate handling and oxidative potential may also become progressively disrupted, perhaps favoring a lower net release of $\mathrm{CO}_{2}$ that contributes to ECAR resulting from carbonic acid buffering in the media (Fig. 6.7). The precise mechanisms at play under these experimental conditions require more sophisticated methods to elucidate fully, but the results of these studies highlight the distinctions between ECAR and glucose utilization that could be leveraged using a multi-sensor approach to study the links between cell metabolism and redox function.

Calculation of metabolite flux ratios (Figs. (6.8E and 6.8F)) further emphasizes this point. Inhibition of mitochondrial ATP production with oligomycin significantly increased the embryo LPR/GCR, ECAR/GCR and ECAR/LPR ratios, consistent with the expected "switch" from glucose-supported OXPHOS to anaerobic glycolysis (lactate fermentation) in order to maintain cellular ATP production. However, maximizing uncoupled mitochondrial respiration and substrate oxidation rates with FCCP decreased ECAR relative to all other metabolite flux rates (Fig. 6.8F), as well as LPR/GCR and OCR/GCR (Fig. 6.8E), reflecting marked shifts in cellular substrate and $\mathrm{H}+$ handling that dissociate elevated rates of glucose and lactate flux from ECAR. The decrease in OCR/GCR, in particular, suggests a greater utilization of non-glucose substrates to support the elevated rates of OCR under these conditions (Fig. 6.7), highlighting the complexity of this 
metabolic system and value of simultaneous monitoring of multiple metabolites for more comprehensive studies of embryo function during development.
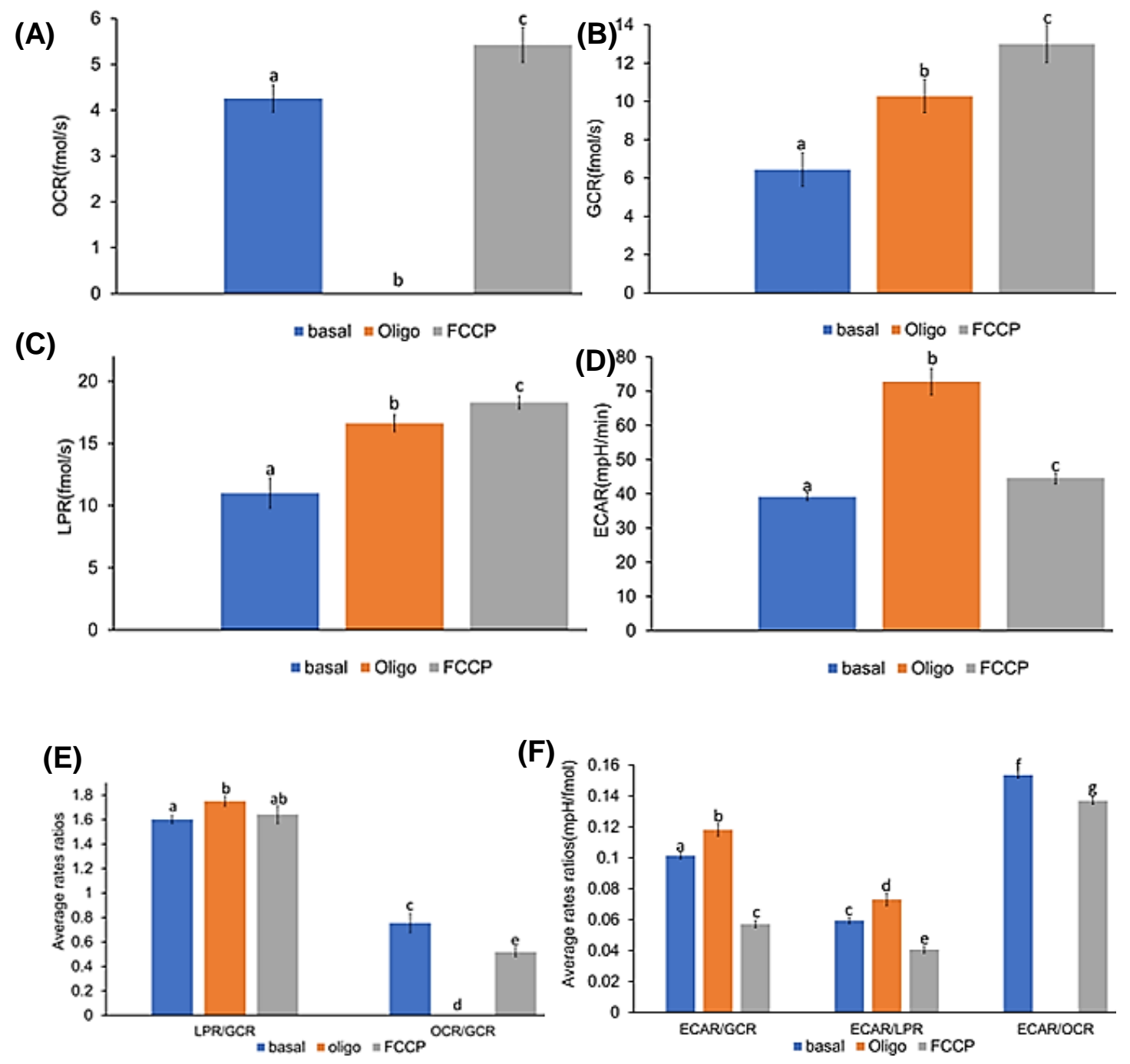

Fig.6.8. Rates for all equine embryos under conditions of (basal, Oligomycin, and FCCP): (A) OCR (B) GCR C) LPR (D) ECAR (E) Flux ratios (LPR/GCR and OCR/GCR). (F) ECAR vs flux ratios (mean $\pm S E, n=11$ ). * paired t-test: $P \leq 0.05$

\subsubsection{Effect of Changing $\mathrm{pH}$ on Glucose and Lactate Measurements}

The glucose and lactate measurements presented in this work were based on the calibration curve obtained assuming a fixed $\mathrm{pH}$ value of the buffer (G-MOPS) at the beginning of the experiments. However, the obtained glucose and lactate measurements have errors due to our ignoring $\mathrm{pH}$ changes during cell basal respiration and glycolysis. 
Using the calibration surfaces in Figs. 6.3A and 6.3B, we examined the amount of errors associated with the glucose and lactate measurements presented in Figs. 6.5-6.8. Table 6.4 shows the maximum errors for each measurement phase.

$\%$ error $=\frac{\text { current at optimal } p H \text { level }- \text { current at correct } p H}{\text { current at optimal } p H} * 100 \%$

Table 6.4: Max percent of error under all conditions of basal, oligo, and FCCP

\begin{tabular}{|l|l|l|l|}
\hline \multicolumn{1}{|c|}{ Analyte } & Basal & \multicolumn{1}{|c|}{ Oligo } & \multicolumn{1}{c|}{ FCCP } \\
\hline Error in \% (glucose measurement) & 6.6 & 6.2 & 9.7 \\
\hline Error in \% (lactate measurement) & 7.09 & 8.3 & 9.7 \\
\hline
\end{tabular}

\subsubsection{Sensor Interference between Glucose and Lactate Sensors}

As discussed above, cross-diffusion of $\mathrm{H}_{2} \mathrm{O}_{2}$ generated at the glucose and lactate sensors is a potential source of interference that could decrease measurement validity if both sensors reside within the same micro-chamber (see Fig. 6.2). The extent of interference could be influenced by substrate concentration at the initiating sensor and the distance between the two sensors in the chamber, so we examined the effect of both factors in studies presented in Fig. 6.9. At concentrations of $0.1 \mathrm{mM}$ and $0.5 \mathrm{mM}$ of the initiating analytes (glucose or lactate), there was no detectable $\mathrm{H}_{2} \mathrm{O}_{2}$ diffusion from initiating to receiving sensors at either distance examined (see D.2.1 in Appendix D). Interference was detectable at distances of $3.2 \mathrm{~mm}$ and $4.6 \mathrm{~mm}$ when analyte concentrations were increased to $1 \mathrm{mM}$ and $2 \mathrm{mM}$, with cross-diffusion times ranging from 40-85 min. There was an interactive effect between analyte concentration and sensor distance, whereby doubling substrate concentration from 1 to $2 \mathrm{mM}$ only significantly shortened diffusion time when sensor distance was decreased from 4.6 to $3.2 \mathrm{~mm}$. These studies indicate that substrate concentration and sensor spacing are potentially important considerations when using enzyme-linked sensors at millimolar substrate concentration in a single micro-chamber, but 
interference can be minimized by running shorter experiments or isolated sensors in satellite sampling chambers as was done in the studies presented in Figs. 6.5-6.6 and 6.8.

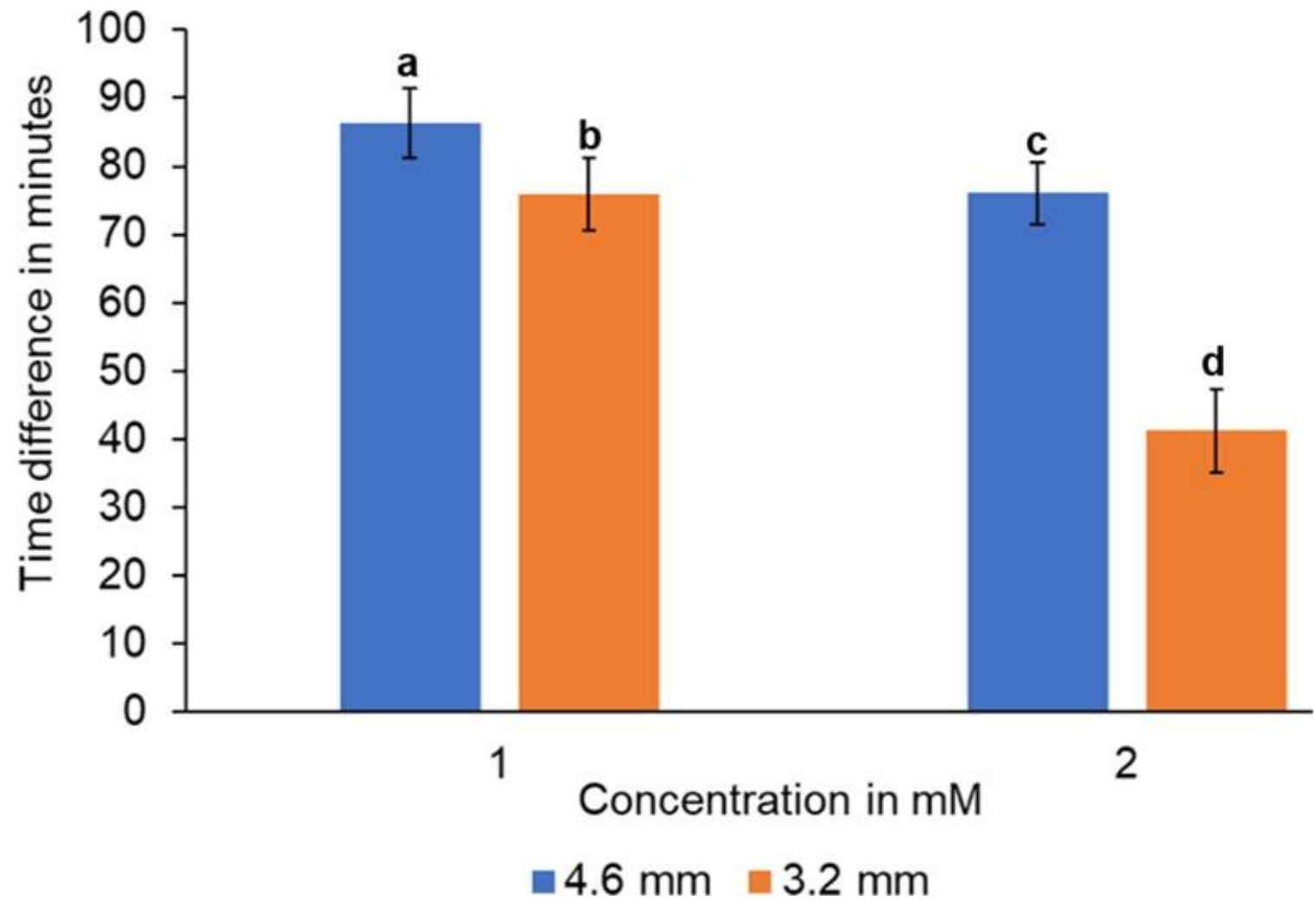

Fig. 6.9 Amount of time ( $\mathrm{min}$ ) vs concentration $(\mathrm{mM})$ at different distances. (mean $\pm S D, n=8$, $\mathrm{P} \leq 0.05)$ 


\section{Chapter 7: Conclusions and Future work}

\subsection{Conclusions}

This work has four main findings: 1) validating the principle of using Clark principle to measure DO; 2) the use of Clark principle to measure oxygen consumption rate of single equine/bovine oocytes and embryos and the respiration associated with proton leak induced by inhibition of the adenosine triphosphate (ATP) synthase (complex V) with oligomycin, and the maximal noncoupled respiratory capacity revealed by FCCP titration; 3) the development of a multi-sensor platform capable of simultaneous measurement of DO concentration, glucose and lactate concentrations in a micro-chamber for real-time evaluation of metabolic flux and the utility for integrative real-time monitoring of aerobic and anaerobic energy metabolism in bovine embryos ; 4) the development of a final multi-sensor platform capable of measurement of DO, glucose, lactate, and ECAR in a micro-chamber for real-time evaluation of metabolic flux in small biological samples. The ability to measure $\mathrm{pH}$ in addition to lactate production can help differentiate the respiratory acid production from glycolytic acidification. We demonstrate the multi-sensor platform's ability to detect a metabolic shift from OXPHOS to glycolysis in equine embryos by an ablation of oxygen consumption and an increase in lactate production as well as ECAR following addition of oligomycin.

The results demonstrated the metabolic features (oxygen and glucose consumption, lactate production, and acidification rate) of equine and bovine embryos as they go from basal respiration to glycolysis. Responses to ATP synthase inhibition and induction of maximal respiration by titration of the protonophore FCCP were measured. The study also presents the sensor interference results between enzymatic sensors in close proximity in a multi-sensor chamber and clearly demonstrated the interference effect as functions of the target analyte concentration and the distance between the corresponding sensors. Limitations of the current setup include challenges associated with handling and positioning of single embryos for analyses, which can 
be overcome by the addition of microscopy and microfluidic channels for sample visualization and manipulation that are currently under development. Some other limitations include the minor toxicity of the enzyme materials on cells in long term development and this need to be avoided. Future applications of this technology could be extended to metabolic monitoring of single cells or small multi-cellular samples obtained from heterogeneous tissues such as tumors or granulomas relevant to the study of cancer and infectious disease, as well as other settings where sample paucity limits direct metabolic assessments by currently available methods.

\subsection{Future Work and Suggestions}

\subsubsection{Electrodes and Chamber New Platform}

In our current setup, we need to move the cell by manual pipetting from one electrode to another to measure specific analyte. Automatic placement of target cells at the right place without human intervention is needed and it can be achieved using advanced microfluidics systems. Other suggestion is to develop a multi-sensor configuration with smaller sensor distances and place the cell on one site close to all sensors without the need to move it from one sensor to another. However, the interference between sensors need to be considered and measured and data need to be corrected based on the level of interference.

\subsubsection{Temperature Control}

Since the applications of our platform include measuring the metabolisms of mammalian cells, the cell environment temperature needs to be controlled. Temperature sensors can be embedded in the platform to ensure a correct temperature reading in the environment chamber during cell metabolism.

\subsubsection{Design of Fully Controlled Circuit to Replace Benchtop}

We have used a benchtop potentiostat for collecting all data in the experiments. The benchtop tends to be expensive and bulky. Designing a fully controlled circuit integrated with the multielectrodes system embedded in a chamber for single cell readings will be more practical and make the use of the design easy and useful for clinical application. Our group is in a process of 
building a complete system to include: multi-sensors, incubator for temperature and gas control, and fully controlled circuit. The design is aimed to be a commercially available system forclinical use. 


\section{Bibliography}

[1] Gardner D.K., Lane M., Stevens J., Schlenker T., Schoolcraft W.B. 2000. Blastocyst score affects implantation and pregnancy outcome: toward a single blastocyst transfer. Fertil Steril, 73, 1155-1158.

[2] Gardner, D.K., Wale, P.L., 2013. Analysis of metabolism to select viable human embryos for transfer. Fertil Steril, 99,1062-1072. DOI: 10.1016/j.fertnstert.2012.12.004.

[3] Eisenreich, W., Heesemann, J., Rudel, T., Goebel, W., 2013. Metabolic host responses to infection by intracellular bacterial pathogens. Front. Cell. Infect. Microbiol, 3, 24. DOI: 10.3389/fcimb.2013.00024.

[4] Yotter, R.A., Wilson, D.M., 2004. Sensor Technologies for Monitoring Metabolic Activity in Single Cells-Part II: Nonoptical Methods and Applications. IEEE sensors journal, 4(4), 412-429. DOI: 10.1109/JSEN.2004.830954.

[5] DeBerardinis, R.J., Lum, J.J., Hatzivassiliou, G., Thompson, C.B., 2008. The biology of cancer: Metabolic reprogramming fuels cell growth and proliferation. Cell Metabolism, 7(1), 11-20.

DOI: 10.1016/j.cmet.2007.10.002.

[6] Lee, H.K., Cho, Y.M., Kwak, S.H., Lim, S., Park, K.S., Shim, E.B., 2009. Mitochondrial dysfunction and metabolic syndrome-looking for environmental factors. Biochim Biophys Acta. Mar,1800, 3, 282-289. DOI: 10.1016/j.bbagen.2009.11.010.

[7] Vanhove, E., Ben-Amor, S., Charlot, S., Colin, D., Devin, A., Rigoulet, M., Sojic, N., Sékli Belaïdi, F., Launay, J., Temple-Boyer, P., Arbault, S., 2013. Development of Electrochemical Microsensors for the Monitoring of Mitochondrial Activities. IEEE, Transducers. Barcelona, SPAIN, DOI: 10.1109/Transducers.2013.6626972.

[8] Brischwein M, Motrescu ER, Cabala E, Otto AM, Grothe H, Wolf B., 2003. Functional cellular assays with multiparametric silicon sensor chips, Lab Chip, 3, 234-240.

[9] Weltin, A. Enderle, B., Kieninger, J.,Urban, G., 2014. Multiparametric, Flexible Microsensor Platform for Metabolic Monitoring In Vivo. IEEE sensors journal. 14, 10.

[10] Eklund, S. E., Taylor, D., Kozlov, E., Prokop, A., Cliffel, D.E., 2004. A microphysiometer for simultaneous measurement of changes in extracellular glucose, lactate, oxygen, and acidification rate. Anal. Chem, 76 (3), 519-527. DOI: 10.1021/ac034641z.

[11] Baumann, W., Lehmann, M., Schwinde, A., Ehret, R., Brischwein, M.,Wolf, B. 1999. Microelectronic sensor system for microphysiological application on living cells. Sensors and Actuators B, 55, 77-89.

[12] Satoh, W., Hosono, H., Yokomaku, H., Morimoto, K., Upadhyay, Suzuki, H., 2008. Integrated Electrochemical Analysis System with Microfluidic and Sensing Functions. Sensors, 8(2), 11111127. DOI: $10.3390 / \mathrm{s} 8021111$.

[13] Martinoia, S., Rosso, N., Grattarola, M., Lorenzelli, L., Margesin, B., Zen, M. 2001. Development of ISFET array-based microsystems for bioelectrochemical measurements of cell populations, Biosensors \& Bioelectronics, 16, 1043-1050. 
[14] Lehmann, M., Baumann, W., Brischwein, M., Gahle, H., Freund, I., Ehret, R., Drechsler, S., Palzer, H., Kleintges, M., Sieben, U., 2001. Simultaneous measurement of cellular respiration and acidification with a single CMOS ISFET. Biosens. Bioelectron, 16, 195-203.

[15] Balcarcel, R.R., and Clark, L.M., 2003. Metabolic screening of mammalian cell cultures using well-plates". Biotechnol Prog, 19, 98-108.

[16] Watanabe, M., Houten, S.M., Mataki, C., Christoffolete, M.A., Kim, B.W., Sato, H., Messaddeq, N., Harney, J.W., Ezaki, O., Kodama, T., Schoonjans, K., Bianco, A.C., Auwerx, J., 2006. Bile acids induce energy expenditure by promoting intracellular thyroid hormone activation. Nature, 439, 484-489. DOI: 10.1038/nature04330.

[17] Sridharan, V., Guichard, J., Bailey, R.M., Kasiganesan, H., Beeson, C., Wright, G.L., 2007. The prolyl hydroxylase oxygen-sensing pathway is cytoprotective and allows maintenance of mitochondrial membrane potential during metabolic inhibition. Am. J. Physiol. Cell Physiol, 292, C719-C728. DOI: 10.1152/ajpcell.00100.2006.

[18] Da-Silva, W.S., Harney, J.W., Kim, B.W., Li, J., Bianco, S.D., Crescenzi, A., Christoffolete, M.A., Huang, S.A., Bianco, A.C., 2007. The small polyphenolic molecule kaempferol increases cellular energy expenditure and thyroid hormone activation. Diabetes, 56, 767-776. DOI:10.2337/db06-1488.

[19] Nicholls, D.G., Johnson-Cadwell, L., Vesce, S., Jekabsons, M., Yadava, N., 2007. Bioenergetics of mitochondria in cultured neurons and their role in glutamate excitotoxicity. J. Neurosci. Res, 85, 3206-3212. DOI: 10.1002/jnr.21290

[20] Bayley, J.P., Devilee, P., 2012. The Warburg effect in 2012. Curr. Opin. Oncol, 24, 62-67. DOI: 10.1097/CCO.0b013e32834deb9e

[21] Hsu, P.P., Sabatini, D.M., 2008. Cancer cell metabolism: Warburg and beyond. Cell, 134, 703-707. DOI: 10.1016/j.cell.2008.08.021

[22] Warburg O., 1956. On the origin of cancer cells. Science, 123: 309-314. DOI: 10.1126/science.123.3191.309.

[23] Warburg, O., 1930. The Metabolism of Tumours: Investigations from the Kaiser Wilhelm Institute for Biology, Berlin-Dahlem Arnold Constable, London.

[24] Pedersen, P.L., 1978. Tumor mitochondria and the bioenergetics of cancer cells, Prog. Exp. Tumor Res, 22, 190-274. DOI: https://doi.org/10.1159/000401202.

[25] Dang, C.V., Semenza, G.L., 1999. Oncogenic alterations of metabolism. Trends Biochem. Sci, 24, 68-72. DOI: https://doi.org/10.1016/S0968-0004(98)01344-9.

[26] Lambert, A.J., Brand, M.D., 2007. Research on mitochondria and aging. Aging Cell, 6, 417420. DOI: 10.1111/j.1474-9726.2007.00316.x

[27] Rolfe, D.F., Brown, G.C., 1997. Cellular energy utilization and molecular origin of standard metabolic rate in mammals. Physiol. Rev, 77, 731-758. DOI: 10.1152/physrev.1997.77.3.731

[28] Butler, M., 2005. Animal cell cultures: recent achievements and perspectives in the production of biopharmaceuticals. Appl. Microbiol. Biotechnol, 68, 283-291. DOI: $10.1007 /$ s00253-005-1980-8 
[29] Owicki, J.C., Parce, J.W., 1992. Biosensors based on the energy metabolism of living cells: the physical chemistry and cell biology of extracellular acidification. Biosens. Bioelectron, 7, 255272. DOI: https://doi.org/10.1016/0956-5663(92)87004-9

[30] Wu, M., Neilson, A., Swift, A.L., Moran, R., Tamagnine, J., Parslow, D., Armistead, S, Lemire, K., Orrell, J,, Teich, J., Chomicz, S., Ferrick, D.A., 2007. Multiparameter metabolic analysis reveals a close link between attenuated mitochondrial bioenergetic function and enhanced glycolysis dependency in human tumor cells. Am. J. Physiol. Cell Physiol, 292, C125-C136. DOI: 10.1152/ajpcell.00247.2006.

[31] Ryan, D., Robards, K. 2006. Metabolomics: The greatest omics of them all?, Anal Chem, 78, 7954-7958.

[32] Christofk, H.R., Vander Heiden, M.G., Harris, M.H., Ramanathan, A., Gerszten, R.E., Wei, R., Fleming, M.D. Schreiber, S.L., Cantley, L.C., 2008. The M2 splice isoform of pyruvate kinase is important for cancer metabolism and tumor growth, Nature, 452, 230-233.

[33] Borini, A., Lagalla, C., Cattoli, M., Sereni, E., Sciajno, R., Flamigni, C., and Coticchio, G. 2005. Predictive factors for embryo implantation potential, Reprod BioMed Online, 10, 653-668.

[34] Reddy, U.M., Wapner, R.J., Rebar, R.W., Tasca, R.J., 2007. Infertility assisted reproductive technology, and adverse pregnancy outcomes: executive summary of a National Institute of Child Health and Human Development workshop. Obstet Gynecol, 109, 967-977.

[35] Sakkas, D., Gardner, D.K., 2005. Noninvasive methods to assess embryo quality, Curr Opin Obstet Gynecol, 17, 283-288.

[36] Leese, H.J., 1991. Metabolism of the preimplantation mammalian embryo. In: Milligan SR, editor. Oxford reviews of reproductive biology. London: Oxford University Press, 13, 35-72.

[37] Wallace, D.C. 2005. Mitochondria and cancer: Warburg addressed. Cold Spring Harb. Symp. Quant. Biol, 70, 363-374.

[38] Gardner, D.K., Phil, D., Lane, M., Stevens, J., Schoolcraft, W.B., 2001. Noninvasive assessment of human embryo nutrient consumption as a measure of developmental potential. Fertil. Steril, 76, 1175-1180. DOI: https://doi.org/10.1016/S0015-0282(01)02888-6.

[39] Urbanski, J.P., Johnson, M.T., Craig, D.D., Potter, D.L., Gardner, D.K., Thorsen, T., 2008. Noninvasive metabolic profiling using microfluidics for analysis of single preimplantation embryos. Anal Chem, 80, 6500-6507.

[40] Lowry, O.H., Passonneau, J.V., 1972. A Flexible System of Enzymatic Analysis. Academic Press, New York.

[41] Leegsma-Vogt, G., Venema, K., and Brouwer, N. et al., 2004. Quantitative on-line monitoring of cellular glucose and lactate metabolism in vitro with slow perfusion. Anal. Chem, 76 (18), $5431-$ 5435 .

[42] Shiku, H., Shiraishi, T., Ohya, H., Matsue, T., Abe, H., Hoshi, H., Kobayashi, M., 2001. Oxygen consumption of single bovine embryos probed by scanning electrochemical microscopy. Anal. Chem, 73, 3751-8.

[43] Ciobanu, M., TaylorJr, D.E., Wilburn, J.P., Cliffel, D.E., 2008. Glucose and Lactate Biosensors for Scanning Electrochemical Microscopy Imaging of Single Live Cells. Ana. Chem, 80(8), 2717-2727. DOI: 10.1021/ac7021184 
[44] Van der Windt, G.J., Chang, C.H., Pearce, E.L., 2016. Measuring Bioenergetics in T Cells Using a Seahorse Extracellular Flux Analyzer. Curr. Protoc. Immunol, 113:3.16B.1-3.16B.14. DOI:

10.1002/0471142735.im0316bs113.

[45] TeSlaa, T., Teitell, M.A., 2014. Techniques to Monitor Glycolysis. Methods in enzymology, 542,91-114.

[46] Janata, J., 1989. Principles of chemical sensors, second edition, 81-236, ISBN: 978-0-38769930-1, DOI: 10.1007/978-0-387-69931-8, Plenum, New York.

[47] Liao, H-K., Chou, J-C., Chung, W-Y., Sun, T-P., Hsiung, S-K., 1998. Study of amorphoustin oxide thin films for ISFET applications. Sensors and Actuators B, 50, 104-109. DOI: https://doi.org/10.1016/S0925-4005(98)00162-2

[48] Yin, L.T., Chou, J.C., Chung, W.Y., Sun, T.P., Hsiung, S.K. 2000. Separate structure extended gate $\mathrm{H}^{+}$-ion sensitive field effect transistor on a glass substrate. Sensors and Actuators $\mathrm{B}, 71$.

[49] McLaughlin, G. W., Braden, K., Franc, B., Kovacs, G. T. A., 2002. Microfabricated solid-state dissolved oxygen sensor. Sens. Actuators B, vol. 83, 138-148

[50] Obeidat, Y., Chen, T., 2016. Characterization of an O2 Sensor Using Microelectrodes. IEEE sensors. Orlando, FL, Oct. 30 - Nov. 2.

[51] Zhu, J., Zhu, Z., Lai, Z., Wang, R., Guo, X., Wu, X., et al., 2002. Planar amperometric glucose sensor based on glucose oxidase immobilized by chitosan film on Prussian blue layer. Sensors, 2,127-136.

[52] Norouzi, P., Faridbod, F., Larijani, B., Ganjali, M.R., 2010. Glucose biosensor based on MWCNTs-Gold nanoparticles in a Nafion film on the glassy carbon electrode using flow injection FFT continuous cyclic voltammetry. Int. J. Electrochem. Sci, 5, 1213-1224.

[53] Weltin, A., Slotwinski, K., Kieninger, J., Moser, I., Jobst, G., Wego, M., Ehret, R., Urban, G.A., 2014. Cell culture monitoring for drug screening and cancer research: A transparent, microfluidic, multi-sensor microsystem., Lab Chip, 14, 138-146. DOI: 10.1039/c3lc50759a.

[54] Clark, L.C.Jr., 1956. Monitor and Control of Blood and Tissue Oxygen Tensions. Trans. Am. Soc. Art. Int. Organs, 2, 41-48.

[55] Jobst, G., Urban, G., Jachimowicz, A., Kohl, F., Tilado, O., Lettenbichler, I., Nauer, G., 1993. Thin-film Clark-type oxygen sensor based on novel polymer membrane systems for in vivo and biosensor applications. Biosens. Bioelectron, 8, 123-128.

[56] Park, J., Chang, J-H., Choi, M., Jungho Pak, J., Lee, D-Y., Kim Pak, Y., 2007. Microfabirated Clark- type Sensor for Measuing Dissolved Oxygen. IEEE Sensors.

[57] Niazi, A., Anthony, C.J., 2014. Development of Oxygen Sensor by Integrating the Low Cost Printed Circuit Board Technology and Solid Electrolyte Membrane. Proceedings of the International Conference on Biomedical Engineering and Systems Prague, Czech Republic, Paper No. 137.

[58] Miyahara, Y., Tsukada, K., Watanabe, Y., Shibata, Y., 1994. Long life planar oxygen sensor. Sensors and Actuators: B. Chemical, 20(23), 89-94. 
[59] Bergveld, P., 1970. Development of an ion sensitive solid-state device for neurophysiological measurements, IEEE Trans. Biomed. Eng, 17, 70-71.

[60] Levine, S., Smith, A.L., 1971. Theory of the differential capacity of the oxide/aqueous electrolyte interface. Discuss. Faraday Soc, 52, 290-301.

[61] Stanley D. Moss, Curtis C. Johnson, Jiri Janata, 1978. Hydrogen, calcium and potassium ion sensitive FET transistors a preliminary report. IEEE Trans. Biomed. Eng. 25, 49-54. DOI:

10.1109/TBME.1978.326377.

[62] Liao, H-K., Chou, J-C., Chung, W-Y., Sun, T-P., Hsiung, S-K., 1998. Study of amorphoustin oxide thin films for ISFET applications. Sensors and Actuators B, 50, 104-109. DOI: https://doi.org/10.1016/S0925-4005(98)00162-2

[63] McConnell, H.M., Owicki, J.C., Parce, J.W., Miller, D.L., Baxter, G.T., Wada, H.G., Pitchford, S., 1992.

The cytosensor microphysiometer:biological applications of silicon technology. Science, 257,1906-1912.

[64] Parce, J. W., Owicki, J.C., Kercso, K.M., Sigal, G.B., Wada, H.G., Muir, V.C., Bousse, L.J., Ross, K.L., Sikic, B.I., McConnell. H.M., 1989. Detection of cell-affecting agents with a silicon biosensor. Science, 246, 243-247.

[65] Rodrigues, N.P., Sakai, Y., Fujii, T., 2007. Cell-Based Microfluidic Biochip for Electrochemical Real-Time Monitoring of Glucose and Oxygen. Transducers- 2007 International Solid-State Sensors, Actuators and Microsystems Conference. Lyon, 843-846.

[66] Yoo, E-H., Lee, S-Y., 2010. Glucose biosensors: An overview of use in clinical practice. Sensors, 10, 4558-4576.

[67] Romero, M.R., Ahumada, F., Garay, F., Baruzzi, A.M., 2010. Amperometric Biosensor for Direct Blood Lactate Detection. Analytical chemistry. 82, 5568-5572.

[68] Arslan, F., Ustabaş, S., Arslan, H., 2011. An Amperometric Biosensor for Glucose Determination Prepared from Glucose Oxidase Immobilized in Polyaniline-Polyvinylsulfonate Film. Sensors (Basel, Switzerland), 11(8), 8152-8163. DOI: http://doi.org/10.3390/s110808152.

[69] Shimomura, T., Sumiya, T., Ono, M., Ito, T., Hanaoka, T.A., 2012. Amperometric L-lactate biosensor based on screen-printed carbon electrode containing cobalt phthalocyanine, coated with lactate oxidase-mesoporous silica conjugate layer. Anal Chim Acta, 714, 114-120.

[70] Rocchitta, G., Spanu, A., Babudieri, S., Latte, G., Madeddu, G., Galleri, G., Nuvoli, S., Bagella, P., Demartis, M.I., Fiore, V., Manetti, R., Serra, P.A., 2016. Enzyme biosensors for biomedical applications: strategies for safeguarding analytical performances in biological fluids. Sensors, 16, 780-801.

[71] Wang, X.D., Wolfbeis, O.S., 2014. Optical methods for sensing and imaging oxygen: Materials, spectroscopies andi applications. Chem. Soc. Rev, 43, 3666-3761. DOI: $10.1039 / \mathrm{c} 4 \mathrm{cs} 00039 \mathrm{k}$.

[72] Bavli, D., Prill, S., Ezra, E., Levy, G., Cohen, M., Vinken, M., Vanfleteren, J.,Jaeger, M., Nahmias, Y., 2016. Real-time monitoring of metabolic function in liver-on-chip microdevices tracks the dynamics of mitochondrial dysfunction. Proc.Natl.Acad.Sci.USA113,E2231-E2240. 
[73] Wu, S., Wu, S., Yi, Z., Zeng, F., Wu, W., Qiao, Y., Zhao, X., Cheng, X., Tian, Y., 2018. Hydrogel-Based Fluorescent Dual pH and Oxygen Sensors Loaded in 96-Well Plates for HighThroughput Cell Metabolism Studies. Sensors, 18, 564. DOI: 10.3390/s18020564.

[74] Pilatus, U., Aboagye, E., Artemov, D., Mori, N., Ackerstaff, E., \& Bhujwalla, Z. M. (2001). Real-time measurements of cellular oxygen consumption, $\mathrm{pH}$, and energy metabolism using nuclear magnetic resonance spectroscopy. Magnetic Resonance in Medicine, 45(5), 749-755. DOI: $10.1002 / \mathrm{mrm} .1102$.

[75] Bennett, B.D., Jetton, T.L., Ying, G., Magnuson, M.A., Piston, D.W., 1996. Quantitative subcellular imaging of glucose metabolism within intact pancreatic islets. J. Biol. Chem., vol. 271, no. 7, pp. 3647-3651. DOI: 10.1063/1.4955155.

[76] Ozawa, A., Sato, Y., Imabayashi, T., Uemura, T., Takagi, J., Sekiguchi, K., 2016. Molecular basis of the ligand binding specificity of _v_8 integrin. J. Biol. Chem., 291, 11551-11565. DOI:

10.1074/jbc.M116.719138

[77] Mookerjee, S.A., Brand, M.D. 2015. Measurement and analysis of extracellular acid production to determine glycolytic rate. J. Vis. Exp., 106; e53464. DOI: [10.3791/53464]

[78] Mark Y. Jeng, Philip A. Hull, Mingjian Fei, Hye-Sook Kwon, Chia-Lin Tsou, Herb Kasler, ChePing Ng, David E. Gordon, Jeffrey Johnson, Nevan Krogan, Eric Verdin, Melanie Ott., 2018. Metabolic reprogramming of human $\mathrm{CD}^{+}$memory $\mathrm{T}$ cells through loss of SIRT1. Journal of Experimental Medicine, 215 (1) 51-62. DOI: 10.1084/jem.20161066

[79] Bibby, S.R., Jones, D.A., Ripley R.M., Urban J.P., 2005. Metabolism of the intervertebral disc: effects of low levels of oxygen, glucose, and $\mathrm{pH}$ on rates of energy metabolism of bovine nucleus $\begin{array}{lllll}\text { pulposus cells. Spine (Phila } \mathrm{Pa} \text { 1976). } & \text { 30(5):487-496. DOI: }\end{array}$ 10.1097/01.brs.0000154619.38122.47

[80] Pemberton, R. M., Cox, T., Tuffin, R., Drago, G. A., Griffiths, J., Pittson, R., Johnson, G., Xu, J., Sage, I. C., Davies, R., Jackson, S. K., Kenna, G., Luxton, R., Hart, J. P., 2014. Fabrication and evaluation of a micro(bio)sensor array chip for multiple parallel measurements of important cell biomarkers. Sensors (Basel, Switzerland), 14(11), 20519-32. DOI: 10.3390/s141120519

[81] Li, C., Shutter, L.A., Wu, P.M., Ahn, C.H. and Narayan, R.K., 2010. Potential of a simple labon-a-tube for point-of-care measurements of multiple analytes. Lab on a Chip,10,11,

1476-1479, DOI: 10.1039/c000897d.

[82] Weltin, A., Slotwinski, K., Kieninger, J., Moser, I., Jobst, G., Wego, M., Ehret, R., Urban, G.A.

Cell culture monitoring for drug screening and cancer research: A transparent, microfluidic,

multi-sensor microsystem., 2014. Lab Chip, 14, 138-146. DOI: 10.1039/c3lc50759a.

[83] Stanley D. Moss, Curtis C. Johnson, Jiri Janata., 1978. Hydrogen, calcium and potassium ion sensitive FET transistors a preliminary report", IEEE Trans. Biomed. Eng. 25, 49-54.

[84] Liao, H-K., Chou, J-C., Chung, W-Y., Sun, T-P., Hsiung, S-K., 1998. Study of amorphoustin oxide thin films for ISFET applications. Sensors and Actuators B., 50, 104-109. DOI: https://doi.org/10.1016/S0925-4005(98)00162-2

[85] Yin, L.T., Chou, J.C., Chung, W.Y., Sun, T.P., Hsiung, S.K.,1998. Characteristics of silicon nitride after $\mathrm{O}_{2}$ plasma treatment for $\mathrm{pH}$ ISFET applications", in: Proceeding of 1998 International 
Electron Devices and Materials Symposia, B, C, National Cheng Kung University, Tainan, Taiwan, ROC, 267-270.

[86] Gimmel, P., Gompf, B., Schemeisser, D., Wiemhofer, H.D., Gopel, W., 1989. $\mathrm{Ta}_{2} \mathrm{O}_{5}$ gates of pH sensitive device comparative spectroscopic and electrical studies, Sensors and Actuators. 17, 195-202.

[87] Pettine, W., Jibson, M., Chen, T., Tobet, S., Nikkel, P., Henry, C.S., 2012. Characterization of Novel Microelectrode Geometries for Detection of Neurotransmitters, IEEE Sensors Journal, vol. 12, no. 5, pp. 1187-1192. DOI: 10.1109/JSEN.2011.2163708

[88] Stanley D. Moss, Curtis C. Johnson, Jiri Janata, 1978. Hydrogen, calcium and potassium ion sensitive FET transistors a preliminary report", IEEE Trans. Biomed. Eng. 25, 49-54. DOI:

10.1109/TBME.1978.326377.

[89] Obeidat, Y., Evans, A., Tedjo, W., Chicco, A., Carnevale, E., Chen, T. 2018a. Monitoring Oocyte/Embryo Respiration Using Electrochemical-Based Oxygen Sensors, Sensors and amp; Actuators: B. Chemical., DOI: https://doi.org/10.1016/j.snb.2018.07.157.

[90] Maclay, G.J. Buttner, W.J., Stetter, J.R., 1988. Microfabricated Amperometric Gas Sensors. IEEE Transactions on Electron Devices. 35, 793-799.

[91] Suzuki, H., Sugama, A., Kojima, N., 1990. Miniature Clark-type oxygen electrode with a threeelectrode configuration. Sens. Actuators B., 2, 297-303.

[92] Compton, G., Laborda, E., Wand, K., 2011. Understanding Voltammetry. 2nd Edition. Imperial College Press.

[93] Ramamoorthy, R., Dutta, P.K. Akbar, S.A., 2003. Oxygen sensors: Materials, methods, designs and applications. J. Mater. Sci., 38, 4271-4282.

[94] Brand, D.M., Nicholls, G.D., 2011. Assessing mitochondrial dysfunction in cells. J. Biochem. 435 (2), 297-312.

[95] Sugimura, S., Matoba, S., Hashiyada, Y., Aikawa, Y., Ohtake, M., Matsuda, H., Kobayashi, S., Konishi, K., Imai, K., 2012. Oxidative phosphorylation-linked respiration in individual bovine oocytes. J. Reprod. Develop., 58(6), 636-641. DOI: https://doi.org/10.1262/jrd.2012-082.

[96] Steinlechner-Maran, R., Eberl, T., Kunc, M., Margreiter, R., Gnaiger, E., 1996. Oxygen dependence of respiration in coupled and uncoupled endothelial cells. Am J Physiol. 271(6 Pt 1), C2053-C2061,

[97] Van der Windt, G.J., Chang, C.H., Pearce, E.L., 2016. Measuring Bioenergetics in T Cells Using a Seahorse Extracellular Flux Analyzer. Curr. Protoc. Immunol., 113:3.16B.1-3.16B.14. DOI:

10.1002/0471142735.im0316bs113.

[98] Thompson, J.G., Partridge, R.J., Houghton, F. D., Cox, C.I., Leese, H.J.,1996. Oxygen uptake and carbohydrate metabolism by in vitro derived bovine embryos. Journal of Reproduction and Fertility., 106(2), 299-306. PMID: 8699414.

[99] Santos, S.C., Kowaltowski, J.A., Bertotti, M., 2017. Single Cell Oxygen Mapping (SCOM) by Scanning Electrochemical Microscopy Uncovers Heterogeneous Intracellular Oxygen Consumption. Sci. Rep., 7, 11428. 
[100] Mills, R.M., Brinster, R.L., 1967. Oxygen consumption of pre-implantation mouse embryos, Experimental Cell Research., 47, 337-344.

[101] Obeidat, Y., Catandi, G., Carnevale, E., Chicco, A., DeMann, A., Field, S., Chen, T.2018b. A Multi-Sensor System for Measuring Bovine Embryo Metabolism, Biosensors and Bioelectronic., DOI: https://doi.org/10.1016/j.bios.2018.09.071.

[102] Guerif, F., McKeegan, P., Leese, H.J., Sturmey, R.J., 2013. A simple approach for consumption and release (CORE) analysis of metabolic activity in single mammalian embryos. PLoS One., 8, e67834. DOI: https://doi.org/10.1371/journal.pone.0067834.

[103] Hardy, K., Hooper, M.A., Handyside, A.H., Rutherford, A.J., Winston, R.M., Leese, H.J., 1989. Non-invasive measurement of glucose and pyruvate uptake by individual human oocytes and preimplantation embryos. Hum Reprod., 4,188-191. PMID: 2918073.

[104] Overstrom, E.W., Duby, R.T., Dobrinsky, J., Roche, J.F., Boland, M.P., 1992. Viability and oxidative metabolism of the bovine blastocyst. Theriogenology., 37, 269 . DOI: https://doi.org/10.1016/0093-691X(92)90338-R.

[105] Wales, R.G., Whittingham, D.G., Hardy, K., Craft, I.L., 1987. Metabolism of glucose by human embryos. J. Reprod. Fertil., 79, 289-297.

[106] Lane, M., Gardner, D.K., 1996. Selection of viable mouse blastocysts prior to transfer using a metabolic criterion. Hum Reprod., 11,1975-1978. PMID: 8921074.

[107] Gott, A.L., Hardy, K., Winston, R.M.L., Leese, H.J., 1990. Non-invasive measurement of pyruvate and glucose uptake and lactate production by single human preimplantation embryos. Hum Reprod. 5,104-108. DOI: https://doi.org/10.1093/oxfordjournals.humrep.a137028.

[108] Leese, H.J., 1992. Metabolism of the preimplantation mammalian embryo. in: Oxford Reviews of Reproductive Biology. Oxford University Press, Oxford, U.K, 13, 35-72.

[109] Rieger, D., Loskutoff, N.M., Betteridge, K.J., 1992a. Developmentally related changes in the metabolism of glucose and glutamine by cattle embryos produced and cocultured in vitro. $\mathrm{J}$ Reprod Fertil., 95(2), 558-595. PMID: 1518013.

[110] Gardner, D.K., Lane, M., Batt, P., 1993. Uptake and metabolism of pyruvate and glucose by individual sheep preattachment embryos developed in vivo". Mol Reprod Dev., 36, 313-319. DOI: https://doi.org/10.1002/mrd.1080360305.

[111] Gardner, D.K., Harvey, A.J., 2015. Blastocyst metabolism. Reprod Fert Dev., 27(4), 638654. DOI: 10.1071/RD14421.

[112] Leese, H.J., 2002. Quiet please, do not disturb: A hypothesis of embryo metabolism and viability. BioEssays., 24, 845-849. DOI: 10.1002/bies.10137.

[113] Leese, H.J., Baumann, C.G., Brison, D.R., McEvoy, T.G., Sturmey, R.G., 2008. Metabolism of the viable mammalian embryo: quietness revisited. Mol Hum Reprod., 14, 667-672. DOI: 10.1093/molehr/gan065

[114] Lane, M., Gardner, D.K., 2005. Mitochondrial malate-aspartate shuttle regulates mouse embryo nutrient consumption. J Biol Chem., 280, 18361-18367. DOI: 10.1074/jbc.M500174200.

[115] Ebner, T., Moser, M., Sommergruber, M., Tews, G., 2003a. Selection based on morphological assessment of oocytes and embryos at different stages of preimplantation development, Hum Reprod Update., 9(3), 251-262. PMID: 12859046. 
[116] Shiku, H., Yasukawa, T., Matsue, T., Ito-Sasaki, T., Yokoo, M., Abe, H., 2007. Oxygen consumption of mammalian embryos and oocytes monitored by scanning electrochemical microscopy. IEEE Sens conference, Atlanta, GA, USA, USA, 28-31. DOI: 10.1109/ICSENS.2007.4388676.

[117] Obeidat, Y., Cheng, M-H., Catandi, G., Carnevale, E., Chicco, A., DeMann, A., Field, S., Chen, T. 2018b. Design of A Multi-Sensor Platform for Integrating Extracellular Acidification Rate with Multi-Metabolite Flux Measurement for Small Biological Samples.

[118] Zhou, Q., Chen, X., 2001. Effects of temperature and pH on the catalytic activity of the immobilized $\beta$-galactosidase from Kluyveromyces lactis. Biochem Eng J 9:33-40.

[119] Arslan, F., Ustabaş, S., Arslan, H., 2011. An Amperometric Biosensor for Glucose Determination Prepared from Glucose Oxidase Immobilized in Polyaniline-Polyvinylsulfonate Film. Sensors (Basel, Switzerland), 11(8), 8152-8163. DOI: http://doi.org/10.3390/s110808152.

[120] Rubinson, K., 2017. Practical corrections for $p(H, D)$ measurements in mixed $\mathrm{H}_{2} \mathrm{O} / \mathrm{D}_{2} \mathrm{O}$ biological buffers. Anal. Meth., 9 (18), 2744-2750. DOI: 10.1039/C7AY00669A.

[121] Romero, N., Swain, P., Neilson, A., Dranka, B., 2017. Improving Quantification of Cellular Glycolytic Rate Using Agilent Seahorse XF Technology. Agilent Technologies, Inc. 121 Hartwell Ave. Lexington, MA 02421.

[122] Mookerjee, S., Brand, M., 2015 Measurement and analysis of extracellular acid production to determine glycolytic rate. J. Vis. Exp. 106; e53464. DOI: 10.3791/53464.

[123] Bó, G.A., Mapletoft, R.J., 2013. Evaluation and classification of bovine embryos. Anim Reprod .10 (3), 344-348.

[124] Krisher, R.L., Prather, R.S., 2012. A role for the Warburg effect in preimplantation embryo development: metabolic modification to support rapid cell proliferation. Mol Reprod Dev.79:311320. DOI: 10.1002/mrd.22037.

[125] Kalhan, S.C. One carbon metabolism in pregnancy: Impact onmaternal, fetal and neonatal health. Mol. Cell. Endocrinol. 2016, 435, 48-60. DOI: 10.1016/j.mce.2016.06.006.

[126] Wales, R.G., Du, Z.F., 1993., Contribution of the pentose phosphate pathway to glucose utilization by preimplantation sheep embryos. Reprod Fertil Dev. 5329-340. DOI: https://doi.org/10.1071/RD9930329.

[127] Rosenzweig, A., Blenis, J., \& Gomes, A. P., 2018., Beyond the Warburg Effect: How Do Cancer Cells Regulate One-Carbon Metabolism? Frontiers in cell and developmental biology, 6, 90. DOI:10.3389/fcell.2018.00090

[128] Ducker, G.S., Rabinowitz, J.D., 2017. One-carbon metabolism in health and disease. Cell Metab 25: 27-42. DOI: 10.1016/j.cmet.2016.08.009.

[129] DeBerardinis, R.J., Chandel, N.S., 2016. Fundamentals of cancer metabolism. Sci. Adv., 2, p. e1600200. DOI: 10.1126/sciadv. 1600200 . 


\section{Appendix A: Modifying enzymes contents to reduce toxicity on cells}

The environment that the oocyte or embryo is exposed to during in vitro measurements can have profound effects on the success of fertilization and subsequent embryo development. To design a system for measuring oocytes or embryos in vitro it is important to make sure that the environment where the cell is tested is healthy and non-toxic and doesn't affect the cell in long term development. Therefore, it is essential to do a toxicity test of any new materials that either have a direct contact with the cell or exist in its environment. Toxicity tests are conducted to evaluate the adverse effects of chemicals or biological substances on cells. In this work, different experiments were done to test the toxicity of some enzyme's materials and their effects on embryos. Photos were taken before and after measuring to show the effect of different additives on the cell's morphology.

\section{A.1 Toxicity of Triton X-100}

Triton is considered a nonionic surfactant that affects cell morphology, membrane permeability, and viability, and it is a common detergent used in biology for protein extraction from cell membranes [82]. If large concentration of Triton is added to the cells or the cell is subject to high amount of Triton, the cells die [83-85]. This toxicity effect arises from the disrupting action of its polar head group on the hydrogen bonding present within the cell's lipid bilayer, and destruction of the compactness and integrity of the lipid membrane will occur.

\section{A.1.1 Triton X-100 Toxicity Test}

Triton was used in glucose and lactate enzymes layers to solubilize the enzymes. Where 5 $\mathrm{mg}$ of GOx and $50 \mathrm{mg}$ of BSA were dissolved in $500 \mu \mathrm{L}$ of $1 \mathrm{mM}$ PBS containing $0.02 \% \mathrm{v} / \mathrm{v}$ Triton. And $2.5 \mathrm{mg}$ of Lox and $50 \mathrm{mg}$ of BSA were dissolved in $500 \mu \mathrm{L}$ of $1 \mathrm{mM}$ PBS containing $0.02 \%$

$\mathrm{v} / \mathrm{v}$ Triton. However, a change on cell morphology and membrane was clearly demonstrated to indicate a toxic effect of Triton on oocytes and embryos which affect the cell life and development. Therefore, to confirm the toxicity of Triton, we have done a toxicity test, ten oocytes were placed 
in a well of $240 \mu \mathrm{L}$ GMOP buffer and $0.02 \%$ Triton for one hour at $38.2^{\circ} \mathrm{C}$. Oocytes were then washed and placed in maturation medium. The next day, the surrounding cells were removed, and the oocyte morphology was examined for normal appearance and extrusion of a polar body (indicating maturation to MII). The oocytes were observed again the following day for delayed maturation. Some oocytes were put on a well with no Triton and had an $84 \%$ maturation rate, meaning that $\sim 8 / 10$ oocytes were alive and extruded a polar body (as we expect and want). While all oocytes in the well that has Triton were dead, and none matured. Photos were taken after putting the cells inside a well that has $0.02 \%$ Triton for 60 min as shown in Fig. A.1 (A), and for oocytes in control well as shown in Fig. A.1 (B).

(A)

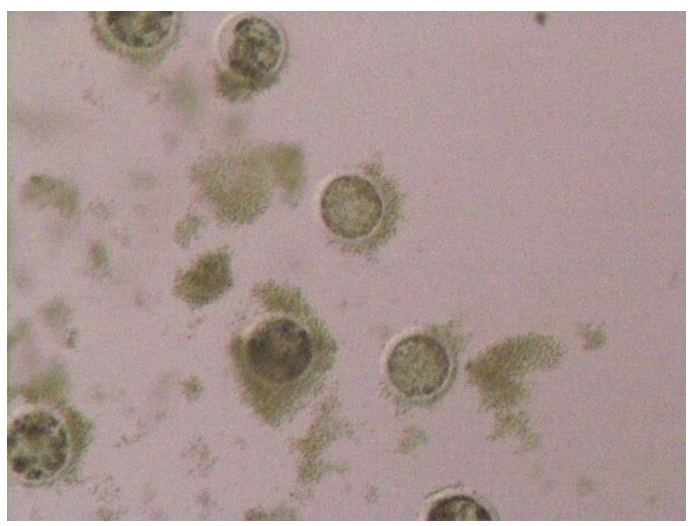

(B)

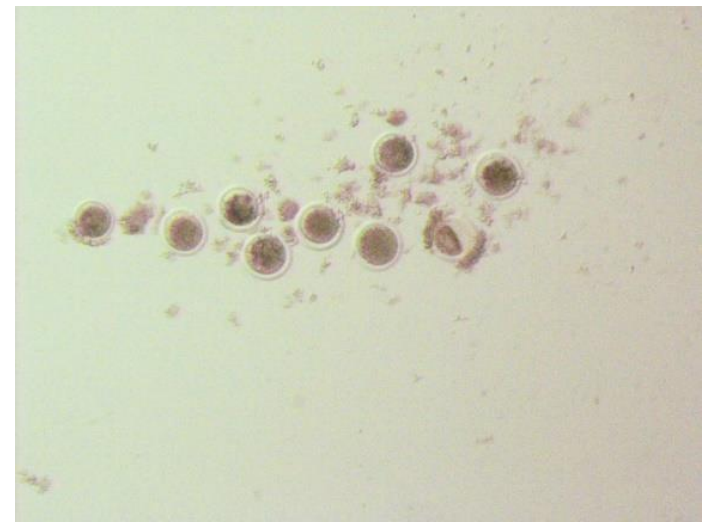

Fig. A.1. (A) Photos of the cells after $60 \mathrm{~min}$ in a well has $0.02 \%$ Triton. (B) Photos of the cells in a control well.

\section{A.2 Toxicity of Glutaraldehyde}

Crosslinking process uses a bifunctional agent to form a bridge between different biocatalytic proteins. Glutaraldehyde is one example of crosslinking reagents that gives great stability to the immobilized enzyme [86]. Glutaraldehyde is a highly reactive chemical that covalently cross-links the albumin molecules to each other and, on application, to the measurement's sites [87]. Glutaraldehyde is widely used as a tissue fixative for histology embryo lung fibroblasts. However, Glutaraldehyde is classified as a toxic substance not only for cells and tissues but repeated exposure to glutaraldehyde causes irritation of eye, nose, throat, or skin resulting in dermatitis 
and asthma [88]. Very low concentration of Glutaraldehyde might lead to significant changes in morphology and density of cells and adding it to different cultured cells had deleterious effects on cell viability [87].

\section{A.2.1 Glutaraldehyde Toxicity Test}

Glutaraldehyde was used in glucose and lactate enzymes layers as a crosslinking reagent to immobilize the enzymes. Where $5 \mathrm{mg}$ of GOx and $50 \mathrm{mg}$ of BSA were thoroughly dissolved in $500 \mu \mathrm{L}$ of $1 \mathrm{mM}$ PBS containing $0.02 \% \mathrm{v} / \mathrm{v}$ Triton and $3 \mu \mathrm{L}$ of $25 \%$ glutaraldehyde solution was added and quickly mixed. And $2.5 \mathrm{mg}$ of Lox and $50 \mathrm{mg}$ of BSA were thoroughly dissolved in 500 $\mu \mathrm{L}$ of $1 \mathrm{mM}$ PBS containing $0.02 \% \mathrm{v} / \mathrm{v}$ Triton and $3 \mu \mathrm{L}$ of $25 \%$ glutaraldehyde solution was added and quickly mixed. However, a damage in the cells was clearly demonstrated after measurement and this indicates the toxic effect of Glutaraldehyde on oocytes and embryos which affect the cell life and development. Therefore, to confirm the toxicity of Glutaraldehyde, we have done a toxicity test, ten oocytes were placed in a well of $240 \mu \mathrm{L}$ GMOP buffer and $1.44 \mu \mathrm{L}$ Glutaraldehyde for one hour at $38.2^{\circ} \mathrm{C}$. Oocytes were then washed and placed in maturation medium. The next day, the surrounding cells were removed, and the oocyte morphology was examined for normal appearance and extrusion of a polar body (indicating maturation to MII). The oocytes were observed again the following day for delayed maturation. Some oocytes were put on a well with no Glutaraldehyde and had an $84 \%$ maturation rate, meaning that $\sim 8 / 10$ oocytes were alive and extruded a polar body (as we expect and want). While all oocytes in the well that has Glutaraldehyde were dead and the surrounding cumulus cells were very hard to remove which might be an indication of fixing, and none matured even the same results happened when 2 layers of mesh were put on top of the Glutaraldehyde layer. Photos were taken after putting the cells inside a well that has Glutaraldehyde for $60 \mathrm{~min}$ as shown in Fig. A.2 (A), and a well that has Glutaraldehyde with 2 layers of mesh as shown in Fig. A.2 (B), and for oocytes in control well as shown in Fig. A.2 (C). 
(A)

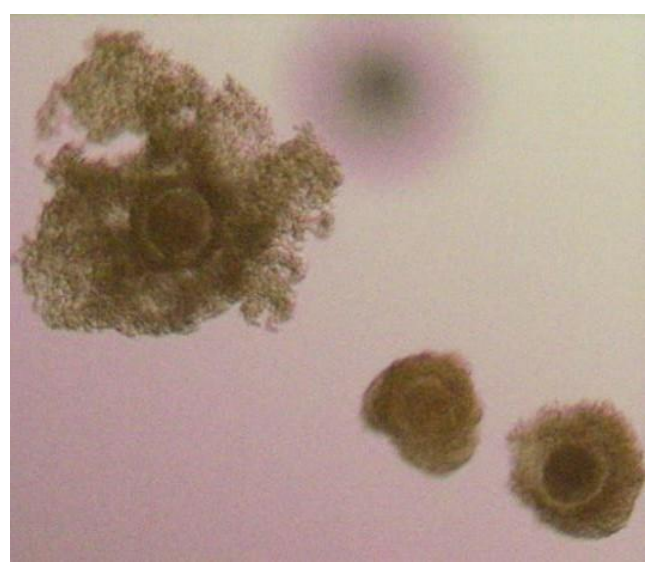

(B)

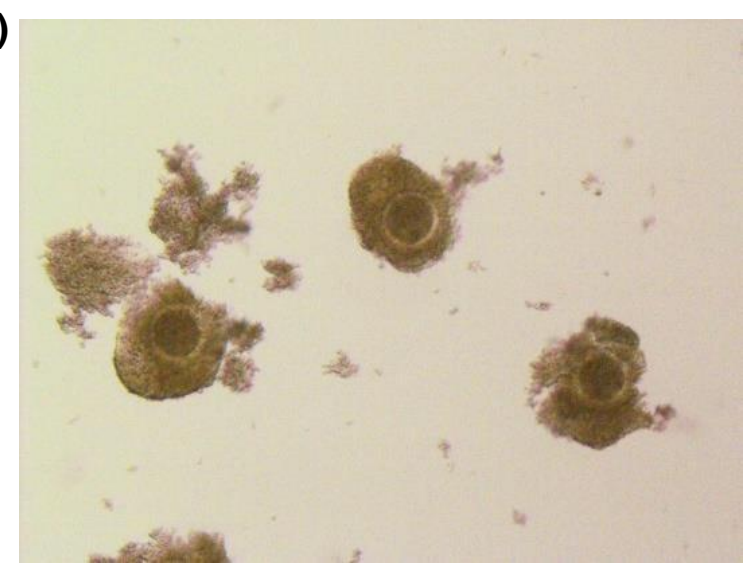

(C)

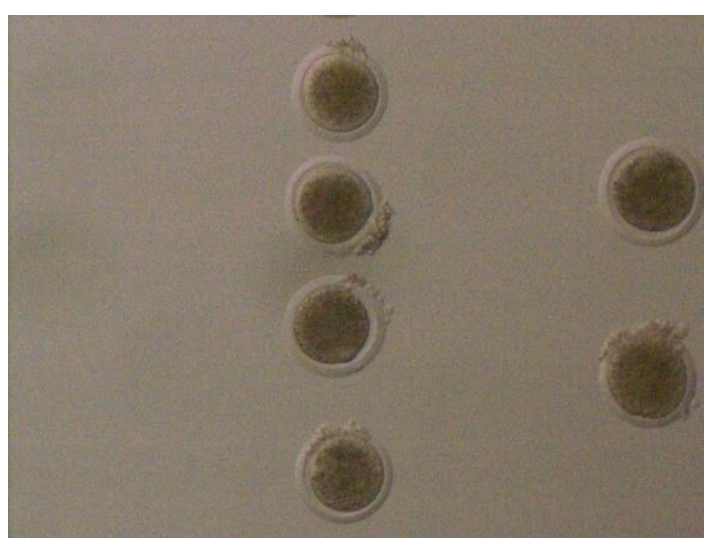

Fig. A.2. (A) Photos of the cells after $60 \mathrm{~min}$ in a well has Glutaraldehyde. (B) Photos in a well that has Glutaraldehyde with 2 layers of mesh. (C) Photos of the cells in a control well.

\section{A.3 Toxicity of Tween-20}

Tween-20 (Polyoxyethylene (20) sorbitan monolaurate) is non-ionic solubilization and stabilizing detergent [89]. Tween-20, as a member of non-ionic surfactants is considered one of the least toxic material with lower irritant potential and it has been introduced in different parts of human life by various industries [89]. Moreover, some in vivo studies showed low toxicity of tween 20, proposing it as a good candidate for application in drug delivery systems as a potential stabilizing agent [90-92].

\section{A.3.1 Tween-20 Toxicity Test}

In this work, Tween-20 was used in glucose and lactate enzymes layers to solubilize and stabilize the enzymes instead of Triton. But before applying that for measurements, toxicity test 
was done to ensure that there is no effect on oocytes or embryos. Ten oocytes were placed in a well of $240 \mu \mathrm{L}$ GMOP buffer and $0.02 \%$ Tween-20 for one hour at $38.2^{\circ} \mathrm{C}$. Oocytes were then washed and placed in maturation medium. The next day, the surrounding cells were removed, and the oocyte morphology was examined for normal appearance and extrusion of a polar body (indicating maturation to MII). The oocytes were observed again the following day for delayed maturation. Some oocytes were put on a well with no Triton and had an $84 \%$ maturation rate, meaning that $\sim 8 / 10$ oocytes were alive and extruded a polar body (as we expect and want). While $80 \%$ of the oocytes in the well that has Tween-20 were alive and matured. Photos were taken after putting the cells inside a well that has $0.02 \%$ Tween-20 for 60 min as shown in Fig. A.3 (A), and for oocytes in control well as shown in Fig. A.3 (B).

(A)

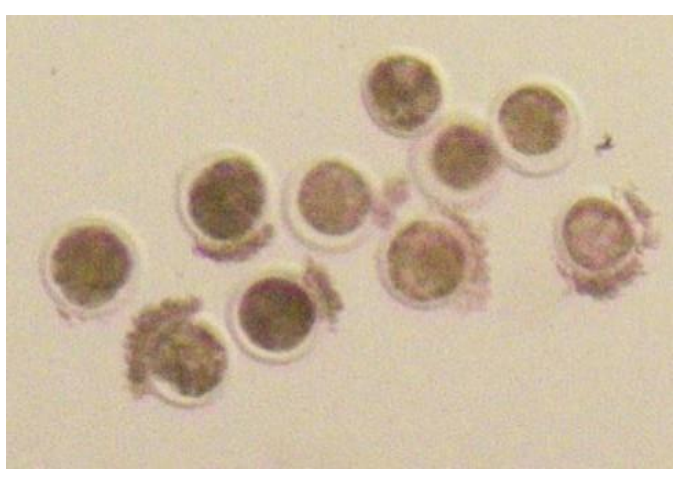

(B)

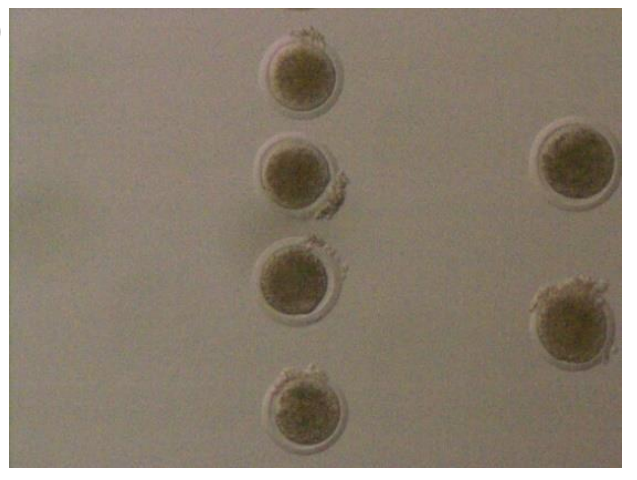

Fig. A.3. (A) Photos of the cells after $60 \mathrm{~min}$ in a well has $0.02 \%$ Tween-20. (B) Photos of the cells in a control well.

Moreover, to confirm that Tween-20 has less toxic effect on cells than other components tested in this chapter, another toxicity test was done for different concentrations of Tween-20. 12 cells were used for each well for all concentrations of $0.01 \%, 0.02 \%, 0.03 \%, 0.04 \%$, and $0.05 \%$, and two control wells. Table A.1 shows the conclusion of the number of the cells that were alive after measurements. 
Table A.1: test of Tween-20 toxicity at different concentrations

\begin{tabular}{|l|l|l|l|}
\hline Treatment & Time in well (min) & \#PB & $\% \mathrm{~PB}$ \\
\hline $0.01 \%$ Tween-20 & 40 & $8 / 12$ & $67 \%$ \\
\hline $0.02 \%$ Tween-20 & 40 & $8 / 12$ & $67 \%$ \\
\hline $0.03 \%$ Tween-20 & 40 & $7 / 12$ & $58 \%$ \\
\hline Control & 40 & $10 / 12$ & $83 \%$ \\
\hline $0.04 \%$ Tween-20 & 50 & $7 / 12$ & $58 \%$ \\
\hline $0.05 \%$ Tween-20 & 50 & $7 / 12$ & $58 \%$ \\
\hline Control & 50 & $10 / 12$ & $83 \%$ \\
\hline
\end{tabular}

Furthermore, Calibration tests were done to see the effect of Tween-20 concentration on enzymes sensors specifications including sensitivity, linearity and LOD. Table A.2 shows the conclusion of the Lactate (L) and Glucose (G) sensors operation when different concentrations of Tween-20 were added to the enzyme's layers. The results show that the increase in Tween-20 concentration didn't improve the sensors properties a lot and considering Table A.1, the increase of Tween-20 concentration might increase the toxicity on cells and need to be avoided to reduce any long-term effect on cells development, therefore we better use a concentration lower than $0.03 \%$; we have used $0.02 \%$ in all experiments.

Table A.2: Lactate and Glucose sensors performance at different Tween-20 concentrations.

\begin{tabular}{|l|l|l|l|l|l|l|}
\hline \multirow{2}{*}{$\begin{array}{l}\text { Tween-20 } \\
\text { concentration in \% }\end{array}$} & Sensitivity $(\mathrm{nA} / \mathrm{mM})$ & Linearity & LOD \\
\cline { 2 - 7 } & $\mathrm{G}$ & $\mathrm{L}$ & $\mathrm{G}$ & $\mathrm{L}$ & $\mathrm{G}$ & $\mathrm{L}$ \\
\hline 0.01 & 12.94 & 12.1 & 0.987 & 0.982 & $0.15 \mathrm{mM}$ & $0.12 \mathrm{mM}$ \\
\hline 0.02 & 13.374 & 14.1 & 0.99 & 0.99 & $0.1 \mathrm{mM}$ & $0.1 \mathrm{mM}$ \\
\hline 0.03 & 13.068 & 14.35 & 0.99 & 0.99 & $0.1 \mathrm{mM}$ & $0.09 \mathrm{mM}$ \\
\hline 0.04 & 13.343 & 14.487 & 0.986 & 0.99 & $0.1 \mathrm{mM}$ & $0.11 \mathrm{mM}$ \\
\hline 0.05 & 14.846 & 14.685 & 0.98 & 0.99 & $0.085 \mathrm{mM}$ & $0.092 \mathrm{mM}$ \\
\hline
\end{tabular}




\section{A.4 Conclusion of the Toxicity Tests and Enzymes Modifications}

To better understand the effect of each components in the enzyme's layers on their performance, calibration tests were done for each of the following cases:

1. No Glutaraldehyde, or Triton, or Tween-20 were added, the enzymes were made only by dissolving their corresponding oxidase and BSA in PBS solution.

2. $0.02 \%$ Triton was added, where $5 \mathrm{mg}$ of $\mathrm{GOx}$ and $50 \mathrm{mg}$ of $\mathrm{BSA}$ were dissolved in $500 \mu \mathrm{L}$ of $1 \mathrm{mM}$ PBS containing $0.02 \% \mathrm{v} / \mathrm{v}$ Triton. And $2.5 \mathrm{mg}$ of Lox and $50 \mathrm{mg}$ of BSA were dissolved in $500 \mu \mathrm{L}$ of $1 \mathrm{mM}$ PBS containing $0.02 \% \mathrm{v} / \mathrm{v}$ Triton.

3. Glutaraldehyde was used in glucose and lactate enzymes layers as a crosslinking reagent to immobilize the enzymes. Where $5 \mathrm{mg}$ of Gox and $50 \mathrm{mg}$ of BSA were thoroughly dissolved in $500 \mu \mathrm{L}$ of $1 \mathrm{mM}$ PBS containing $0.02 \% \mathrm{v} / \mathrm{v}$ Triton and $3 \mu \mathrm{L}$ of $25 \%$ glutaraldehyde solution was added and quickly mixed. And $2.5 \mathrm{mg}$ of Lox and $50 \mathrm{mg}$ of BSA were thoroughly dissolved in $500 \mu \mathrm{L}$ of $1 \mathrm{mM}$ PBS containing $0.02 \% \mathrm{v} / \mathrm{v}$ Triton and 3 $\mu \mathrm{L}$ of $25 \%$ glutaraldehyde solution was added and quickly mixed.

4. Tween-20 was used, where $5 \mathrm{mg}$ of Gox and $50 \mathrm{mg}$ of BSA were dissolved in $500 \mu \mathrm{L}$ of $1 \mathrm{mM}$ PBS containing $0.02 \% \mathrm{v} / \mathrm{v}$ Tween-20. And $2.5 \mathrm{mg}$ of Lox and $50 \mathrm{mg}$ of BSA were dissolved in $500 \mu \mathrm{L}$ of $1 \mathrm{mM}$ PBS containing $0.02 \% \mathrm{v} / \mathrm{v}$ Tween-20.

Table A.3 shows the conclusion of the sensor's performance under different contents of enzymes layers. Comparing the performance of the sensors with different enzymes layers materials, it was found that the use of Glutaraldehyde has the best sensitivity, good linearity and LOD. However, Glutaraldehyde is very toxic on oocytes and embryos and can't be added to the enzymes as demonstrated in Section A.2. Although the sensitivities when Tween-20 and no Glutaraldehyde was used were lower than when Glutaraldehyde was used, the sensors still show good and comparable performance with good linearity and LOD and better performance than the case when Triton only or no-additives were used. Therefore, we have decided to use Tween-20 
instead of Triton for its high performance and less toxicity effect on oocytes and embryos as demonstrated in Section A.3.

Table A.3: Sensors performance under different contents of enzymes layers

\begin{tabular}{|c|c|c|c|c|c|c|c|c|c|}
\hline \multirow[t]{2}{*}{\begin{tabular}{|l} 
Additive \\
\end{tabular}} & \multicolumn{2}{|c|}{ Linearity } & \multicolumn{2}{|c|}{$\begin{array}{l}\text { Sensitivity } \\
(\mathrm{nA} / \mathrm{mM})\end{array}$} & \multicolumn{2}{|c|}{$\begin{array}{l}\text { LOB }=\text { mean }_{\text {blank }} \\
+\mathrm{SD}_{\text {blank }}\end{array}$} & \multicolumn{2}{|c|}{$\begin{array}{l}\mathrm{LOD}= \\
1.5^{\star} \mathrm{LOB} / \text { slope }\end{array}$} & \multirow[t]{2}{*}{$\begin{array}{l}\text { Effect on } \\
\text { cell }\end{array}$} \\
\hline & $\begin{array}{l}\text { Lact } \\
\text { ate }\end{array}$ & $\begin{array}{l}\text { Gluc } \\
\text { ose }\end{array}$ & $\begin{array}{l}\text { Lact } \\
\text { ate }\end{array}$ & $\begin{array}{l}\text { gluco } \\
\text { se }\end{array}$ & Lactate & $\begin{array}{l}\text { glucos } \\
\mathrm{e}\end{array}$ & Lactate & $\begin{array}{l}\text { Gluco } \\
\text { se }\end{array}$ & \\
\hline $\begin{array}{l}\text { No } \\
\text { additives }\end{array}$ & $\begin{array}{l}0.98 \\
5\end{array}$ & 0.984 & 9.9 & 6.6 & 2.8 & 1.8 & $\begin{array}{l}0.4 \\
\mathrm{mM}\end{array}$ & $\begin{array}{l}0.4 \\
\mathrm{mM}\end{array}$ & No effect \\
\hline Triton & 0.99 & 0.99 & 9.79 & 9.64 & 1.7 & 1.73 & $\begin{array}{l}0.27 \\
\mathrm{mM}\end{array}$ & $\begin{array}{l}0.49 \\
\mathrm{mM}\end{array}$ & $\begin{array}{l}\text { It kills the } \\
\text { cell }\end{array}$ \\
\hline \begin{tabular}{|l|} 
Tween-20 \\
\end{tabular} & 0.99 & 0.99 & $\begin{array}{l}14.3 \\
8\end{array}$ & 14.1 & 1.025 & 1.09 & $\begin{array}{l}0.1 \\
\mathrm{mM}\end{array}$ & $\begin{array}{l}0.1 \\
\mathrm{mM}\end{array}$ & $\begin{array}{l}\text { Appears } \\
\text { fine }\end{array}$ \\
\hline $\begin{array}{l}\text { Glutaralde } \\
\text { hyde }\end{array}$ & 0.99 & 0.99 & $\begin{array}{l}21.2 \\
38\end{array}$ & 16.5 & 1.53 & 1.4 & $0.1 \mathrm{mM}$ & $\begin{array}{l}0.1 \\
\mathrm{mM}\end{array}$ & $\begin{array}{l}\text { It kills the } \\
\text { cell }\end{array}$ \\
\hline
\end{tabular}




\section{Appendix B: Bovine Embryos Grading, Experiment Setup, Statistical Analysis and Sensors Properties}

\section{B.1 Embryo Morphological Grading}

In vitro produced bovine embryos of good quality and different stages of development were selected for multi-sensor measurements. Embryos were evaluated using a stereomicroscope at $100 \mathrm{X}$ magnification. Embryos were classified in 5 stages of normal development associated with days in culture: 8- to16-cell embryos, morulae (compact mass of approximately 62 cells), blastocysts (formation of blastocoel), expanded blastocysts (continued expansion of the blastocoel), and hatched blastocysts (escape of the embryo from the surrounding zona pellucida). Embryos were qualitative graded based on morphology using the International Embryo Transfer Society (IETS) scoring system, which classifies excellent embryos as 1 and dead or degenerating embryos as 4 [123]. Embryos of morphologic grades 1 or 2, were considered to be excellent or good in quality and have good developmental potential; these embryos were used for multi-sensor measurements. Embryos of poor-quality (grade 3) and uncertain viability were not used. Embryos graded as 4 (degenerate or arrested in development) were used as negative controls as well as dead, uncleaved oocytes. Just prior to multi-sensor measurements, embryos were taken out of an incubator $\left(38.5{ }^{\circ} \mathrm{C}, 5 \% \mathrm{CO}_{2}, 5 \% \mathrm{O}_{2}, 90 \% \mathrm{~N}_{2}\right.$ ), evaluated and selected. The selected embryo was moved to a dish containing G-MOPS and held at $38.5^{\circ} \mathrm{C}$ until being placed into the multisensor chamber.

\section{B.2 Experiment Setup}

All experiments were done in ERL. Temperature were maintained by setting a hotplate at $38.8^{\circ} \mathrm{C}$. the hotplate is a flat base connected under a microscope (Fig. B.1). The measurements were done using a benchtop potentostat. The data collected by the potentiostat was analyzed using a set of custom-built tools written in Matlab. 


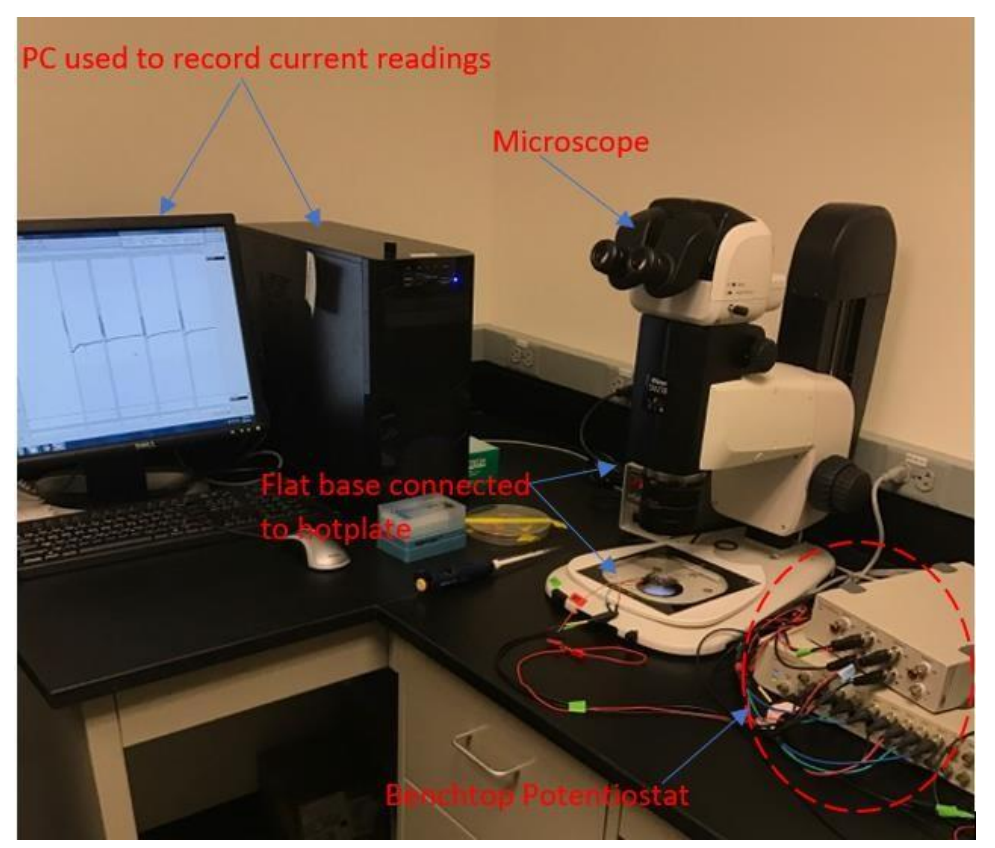

Fig. B.1. Experiment setup for measuring single embryo metabolism

\section{B.3 Statistical Analysis}

One-way ANOVA was used to analyze the pattern of oxygen consumption, glucose uptake, and lactate production during bovine embryos development from (8 to 32) cells stage to hatched blastocyst. Differences between means were examined using Tukey HSD (pairwise comparison among stages). These statistical analyses are for the data in Chapter $\mathbf{5}$ and similar methods were used for statistical analysis of the data in Chapter 6.

Table B.1: Oxygen consumption, glucose uptake, and lactate production of in vitro produced bovine embryos. One-way ANOVA for all data is $(P<0.001)$. a, b, c, d within columns: Tukey HSD (pairwise comparison), values with different superscripts are significantly different ( $P \leq$ 0.05).

\begin{tabular}{|l|l|l|l|}
\hline \multicolumn{1}{|c|}{ stage } & \multicolumn{1}{|c|}{$\begin{array}{c}\text { Oxygen consumption } \\
(\mathrm{fmol} / \mathrm{s}) \pm \mathrm{SD}\end{array}$} & $\begin{array}{c}\text { Glucose uptake } \\
(\mathrm{fmol} / \mathrm{s}) \pm \mathrm{SD}\end{array}$ & $\begin{array}{c}\text { Lactate } \\
\text { production } \\
(\mathrm{fmol} / \mathrm{s}) \pm \mathrm{SD}\end{array}$ \\
\hline Dead cells (12) & $0.09 \pm 0.10^{\mathrm{a}}$ & $0.13 \pm 0.09^{\mathrm{a}}$ & $0.15 \pm 0.10^{\mathrm{a}}$ \\
\hline 8-32 cells (12) & $0.77 \pm 0.20^{\mathrm{b}}$ & $1.12 \pm 0.23^{\mathrm{b}}$ & $1.53 \pm 0.24^{\mathrm{b}}$ \\
\hline Morula (7) & $1.18 \pm 0.15^{\mathrm{bc}}$ & $1.65 \pm 0.23^{\mathrm{bc}}$ & $2.17 \pm 0.19^{\mathrm{bc}}$ \\
\hline Blastocyst (6) & $1.48 \pm 0.30^{\mathrm{c}}$ & $2.26 \pm 0.26^{\mathrm{c}}$ & $2.77 \pm 0.27^{\mathrm{c}}$ \\
\hline Expanded blastocyst (17) & $1.94 \pm 0.30^{\mathrm{d}}$ & $2.92 \pm 0.49^{\mathrm{d}}$ & $3.74 \pm 0.50^{\mathrm{d}}$ \\
\hline Hatched blastocyst (8) & $3.58 \pm 0.37^{\mathrm{e}}$ & $5.63 \pm 0.55^{\mathrm{e}}$ & $6.96 \pm 0.66^{\mathrm{e}}$ \\
\hline
\end{tabular}



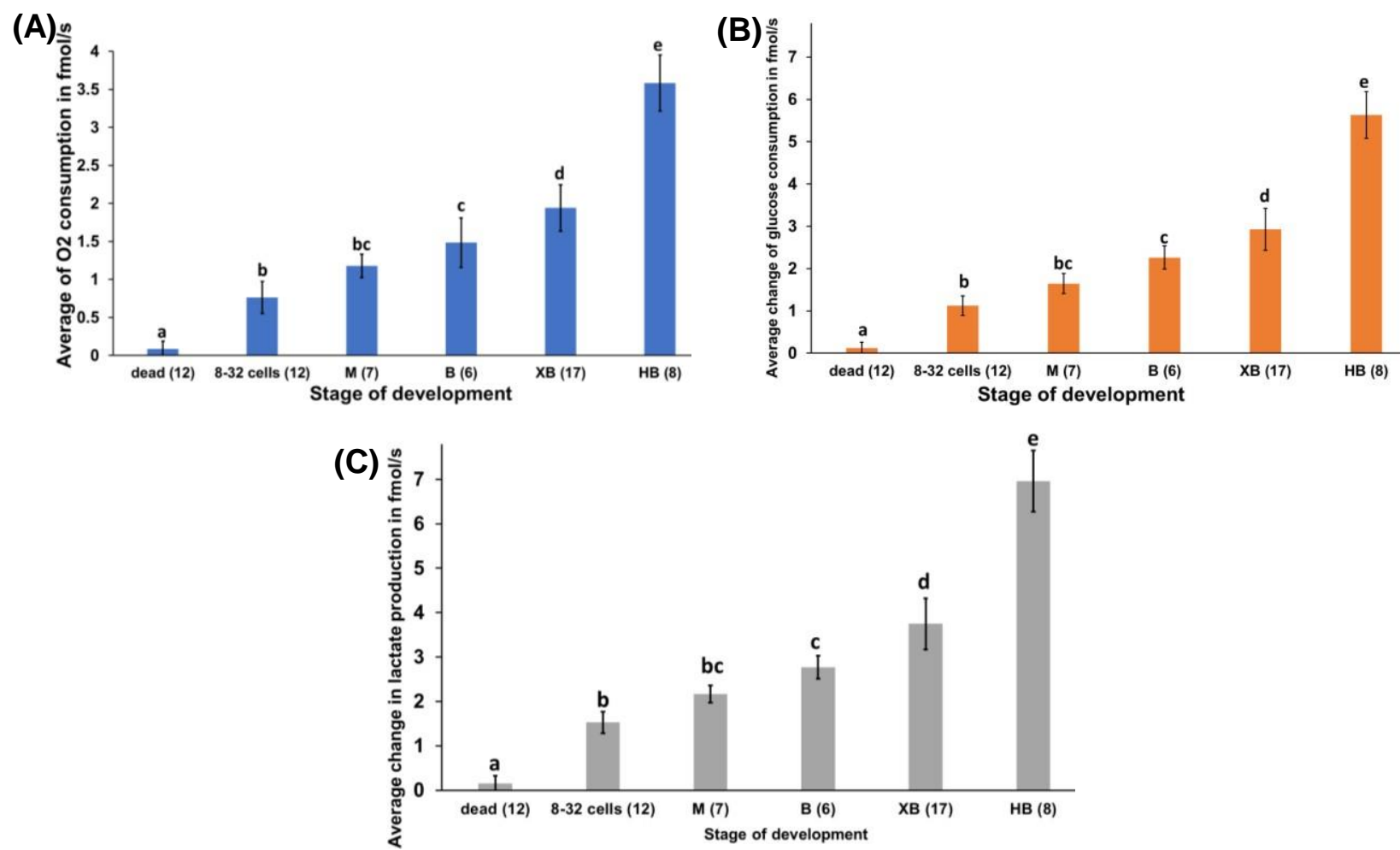

Fig. B.2. (A) Oxygen consumption with development. (B) Glucose consumption with development. (C) lactate production with development. a, b, c, d within columns Tukey HSD (pairwise comparison), values with different superscripts are significantly different $(P \leq 0.05)$.

\section{B.4 Sensors Properties}

The responsiveness and reproducibility of the sensors used in Chapter $\mathbf{5}$ and Chapter $\mathbf{6}$ are described in this section. Since all sensors were tested using a quasi RE and this might cause a problem, the use of quasi RE is also described.

\section{B.4.1 Sensors Responsiveness and Reproducibility}

\section{B.4.1.1 Oxygen Sensor Responsiveness and Reproducibility}

The responsiveness and reproducibility of the oxygen sensor signal to changing oxygen concentrations was demonstrated by adding G-MOPS $\left(21 \%\right.$ or $158 \mu \mathrm{M} \mathrm{O}_{2}$ ) and $0.1 \mathrm{M} \mathrm{Na}_{2} \mathrm{SO}_{3}$ (an $\mathrm{O}_{2}$ chelator) in a sequential cycle. In each step, a $0.1 \mathrm{M} \mathrm{Na}_{2} \mathrm{SO}_{3}$ solution with zero oxygen concentration was added using a pipette and reduction current was measured. Thenthe solution was removed, and the electrodes dried before adding a saturated G-MOPS medium to measure 
the reduction current corresponding to high oxygen concentration. The process was repeated multiple times to ensure sensor repeatability.

Results demonstrated a $0 \%$-to- $21 \%$ oxygen response time of $30 \mathrm{~s}$. The sensor also showed good reproducibility, with a mean of $-297 \mathrm{nA}$ and a standard deviation of ( $3 \mathrm{nA}$ or $1.18 \mu \mathrm{M})$ at the saturated dissolved oxygen level in G-MOPS medium $(21 \%$ or $158 \mu \mathrm{M})$, and a mean of $(-2.01 \mathrm{nA}$ or $1.1 \mu \mathrm{M})$ with standard deviation of $(0.45 \mathrm{nA}$ or $0.121 \mu \mathrm{M})$ at the minimum oxygen level $(0.315 \%$ or $2.37 \mu \mathrm{M}$ ) using $\mathrm{Na}_{2} \mathrm{SO}_{3}$ (Fig. B.3).

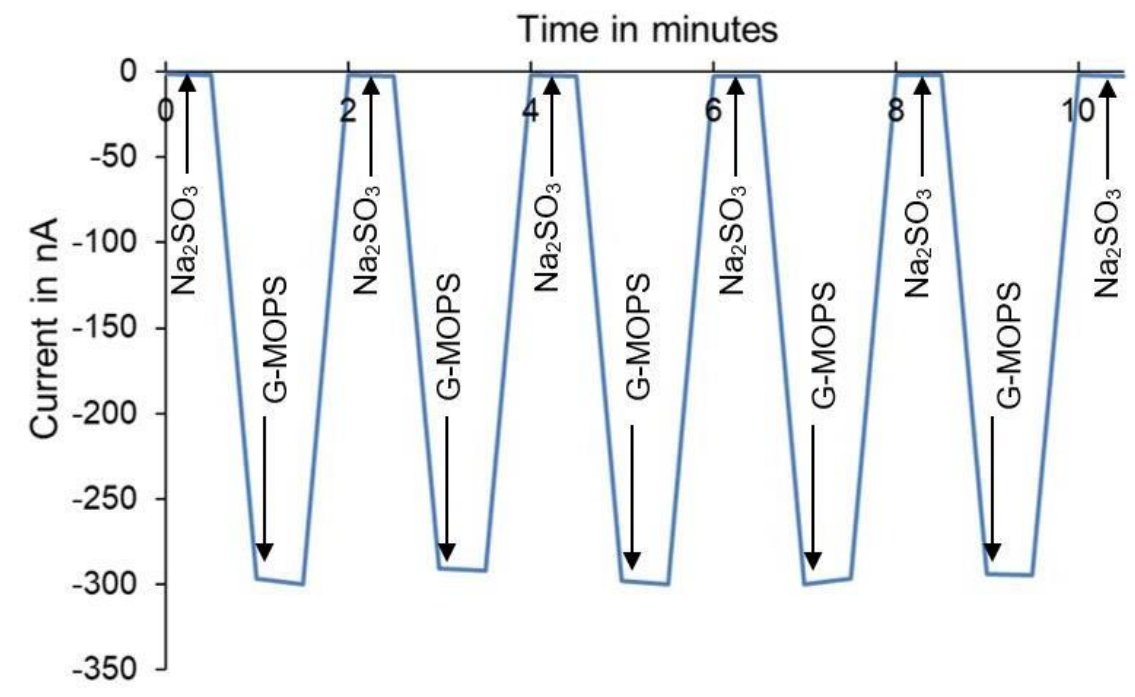

Fig. B.3. Time response and reproducibility of the oxygen sensor.

\section{B.4.1.2 Glucose Sensor Responsiveness and Reproducibility}

The responsiveness and reproducibility of the glucose sensor signal to changing glucose concentrations was demonstrated by adding $9 \mathrm{mM}$ glucose and DI water (zero glucose) in a sequential cycle. In each step, a DI water with zero glucose concentration was added using a pipette and oxidation current was measured. Then the solution was removed, and theelectrodes dried before adding a $9 \mathrm{mM}$ glucose to measure the oxidation current corresponding to high glucose concentration. The process was repeated multiple times to ensure sensor repeatability. 
Results demonstrated a low to high glucose response time of $40 \mathrm{~s}$. The sensor also showed good reproducibility, with a mean of $130.4 \mathrm{nA}$ and a standard deviation of $1.76 \mathrm{nA}$ at the max glucose level and a mean of $2.3 \mathrm{nA}$ with standard deviation of $0.5 \mathrm{nA}$ at the zero-glucose level. (Fig. B.4).

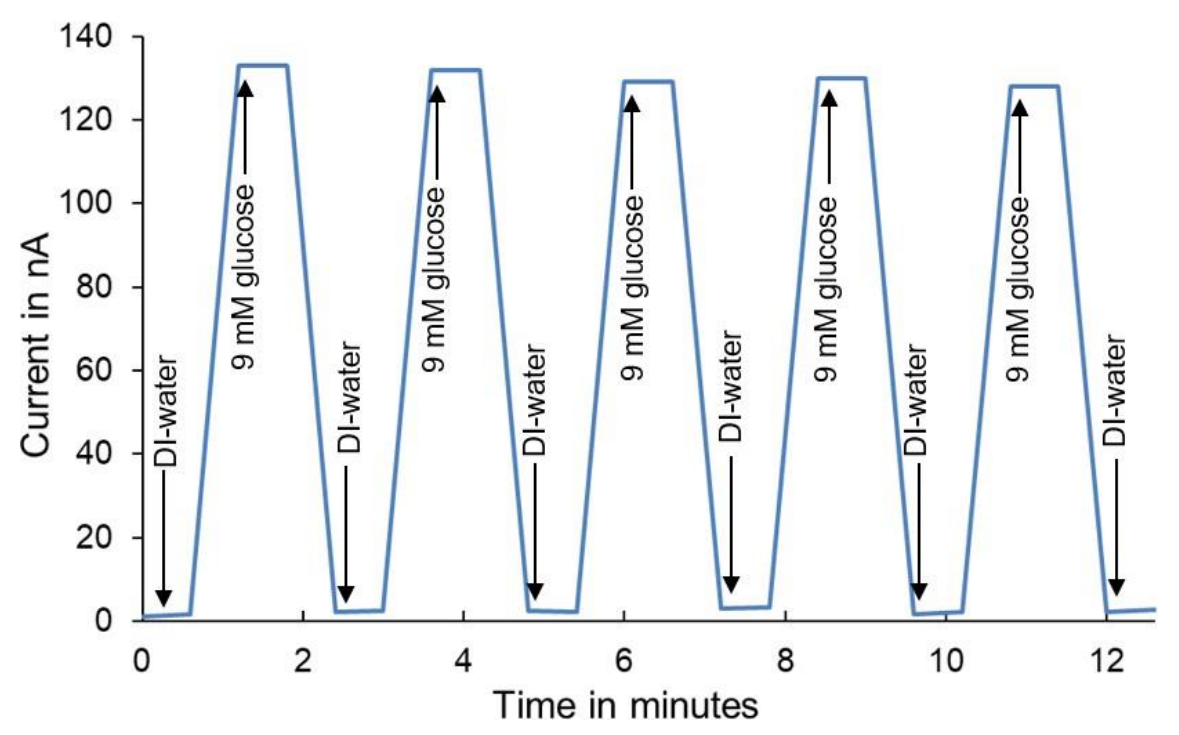

Fig. B.4. Time response and reproducibility of glucose sensor.

\section{B.4.1.3 Lactate Sensor Responsiveness and Reproducibility}

The responsiveness and reproducibility of the lactate sensor signal to changing lactate concentrations was demonstrated by adding $6 \mathrm{mM}$ lactate and DI water (zero lactate) in a sequential cycle. In each step, a DI water with zero lactate concentration was added using a pipette and oxidation current was measured. Then the solution was removed, and the electrodes dried before adding a $6 \mathrm{mM}$ lactate to measure the oxidation current corresponding to high lactate concentration. The process was repeated multiple times to ensure sensor repeatability.

Results demonstrated a low to high lactate response time of $40 \mathrm{~s}$. The sensor also showed good reproducibility, with a mean of $124.75 \mathrm{nA}$ and a standard deviation of $1.8 \mathrm{nA}$ at the max lactate level and a mean of $2.5 \mathrm{nA}$ with standard deviation of $0.43 \mathrm{nA}$ at the zero-lactate level. (Fig. B.5). 


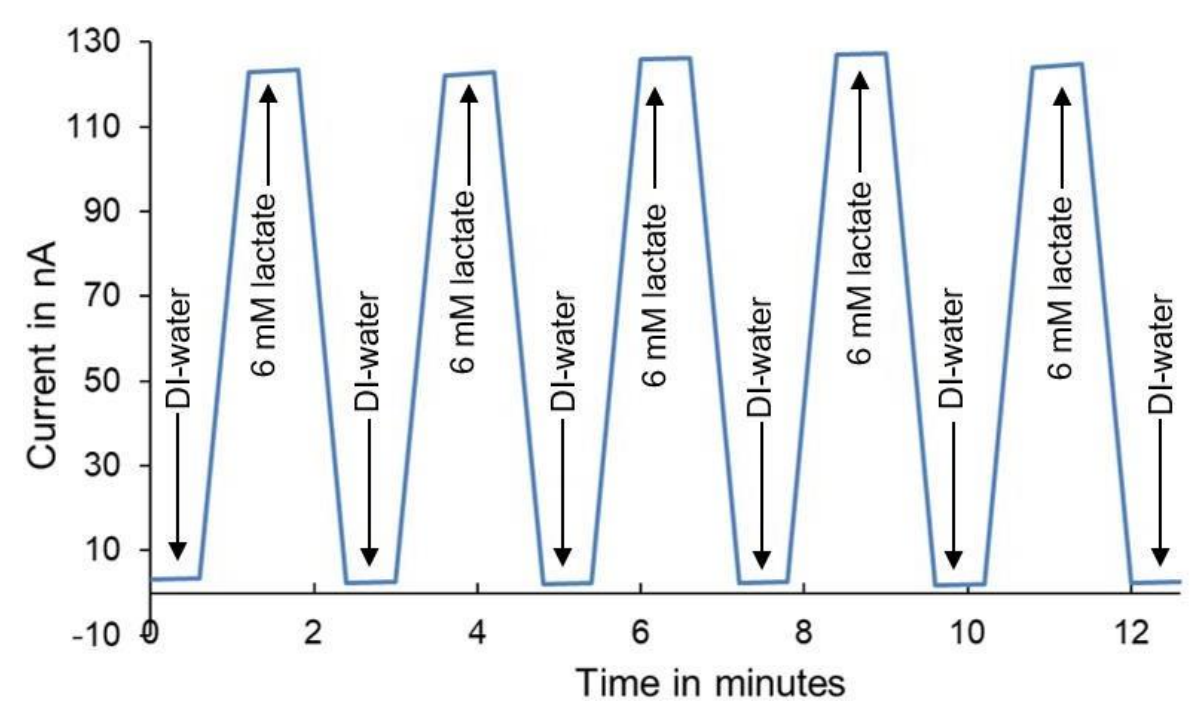

Fig. B.5. Time response and reproducibility of the lactate sensor.

\section{B.4.2 The Use of Quasi-Reference Electrode}

The major advantages of using $\mathrm{Ag} / \mathrm{AgCl}$ reference electrode is twofold: material stability over time and minimized polarization at the electrode surface which results in minimized potential shift between the reference and the working electrodes. The use of Au as reference electrode material for our multi-sensor setup is mainly due to 1) manufacturing compatibility and simplicity of the sensor system. The electrode substrate (glass substrate) of our multi-sensor system is set up to be disposable. Therefore, keeping the overall manufacturing costs down using the same material for all electrodes in the system is one of the main goals. 2) The advantage of stability using $\mathrm{Ag} / \mathrm{AgCl}$ reference electrode is greatly reduced due to the fact that our multi-sensor electrode substrate is intended to be one-time use only. 3) The potential shift of quasi-reference electrode such as Au electrode is insignificant in the short time period. In fact, the following table illustrates the similarity of activation voltages using $\mathrm{Ag} / \mathrm{AgCl}$ and $\mathrm{Au}$ in a three-electrode setup at the beginning of the usage period. The potential shift over longer period of time due to surface polarization does not impact our system due to the one-time-use nature of our system. 
Table B.2. Comparison of activation voltages of $\mathrm{Ag} / \mathrm{AgCl}$ and $\mathrm{Au}$ reference electrodes

\begin{tabular}{|l|l|l|l|l|l|}
\hline Ref\# & System & RE material & $\begin{array}{l}\text { Activation } \\
\text { voltage for } \\
\text { oxygen sensing }\end{array}$ & Linearity & $\begin{array}{l}\text { Response } \\
\text { time }\end{array}$ \\
\hline$[91]$ & $\begin{array}{l}\text { Three or Two } \\
\text { electrodes }\end{array}$ & $\mathrm{Au}$, or $\mathrm{Ag} / \mathrm{AgCl}$ & -0.6 to -0.8 & good & $\begin{array}{l}\mathrm{Ag} / \mathrm{AgCl} \\
20-50 \mathrm{~s} \\
\mathrm{Au}: 30- \\
180 \mathrm{~s}\end{array}$ \\
\hline$[56]$ & Three electrodes & $\mathrm{Ag} / \mathrm{AgCl}$ & -0.6 to -0.9 & 0.99 & $40 \mathrm{~s}$ \\
\hline$[57]$ & Three electrodes & $\mathrm{Au}$ & -0.8 to -1 & good & \\
\hline$[49]$ & Three electrodes & $\mathrm{Au}$ & -0.6 to -0.7 & $\geq 0.99$ & \\
\hline $\begin{array}{l}{[101,} \\
117]\end{array}$ & Three electrodes & $\mathrm{Au}$ & -0.55 to -0.7 & 0.98 & $30 \mathrm{~s}$ \\
\hline
\end{tabular}

\section{B.4.3 pH sensor Calibration (pH6-pH8)}

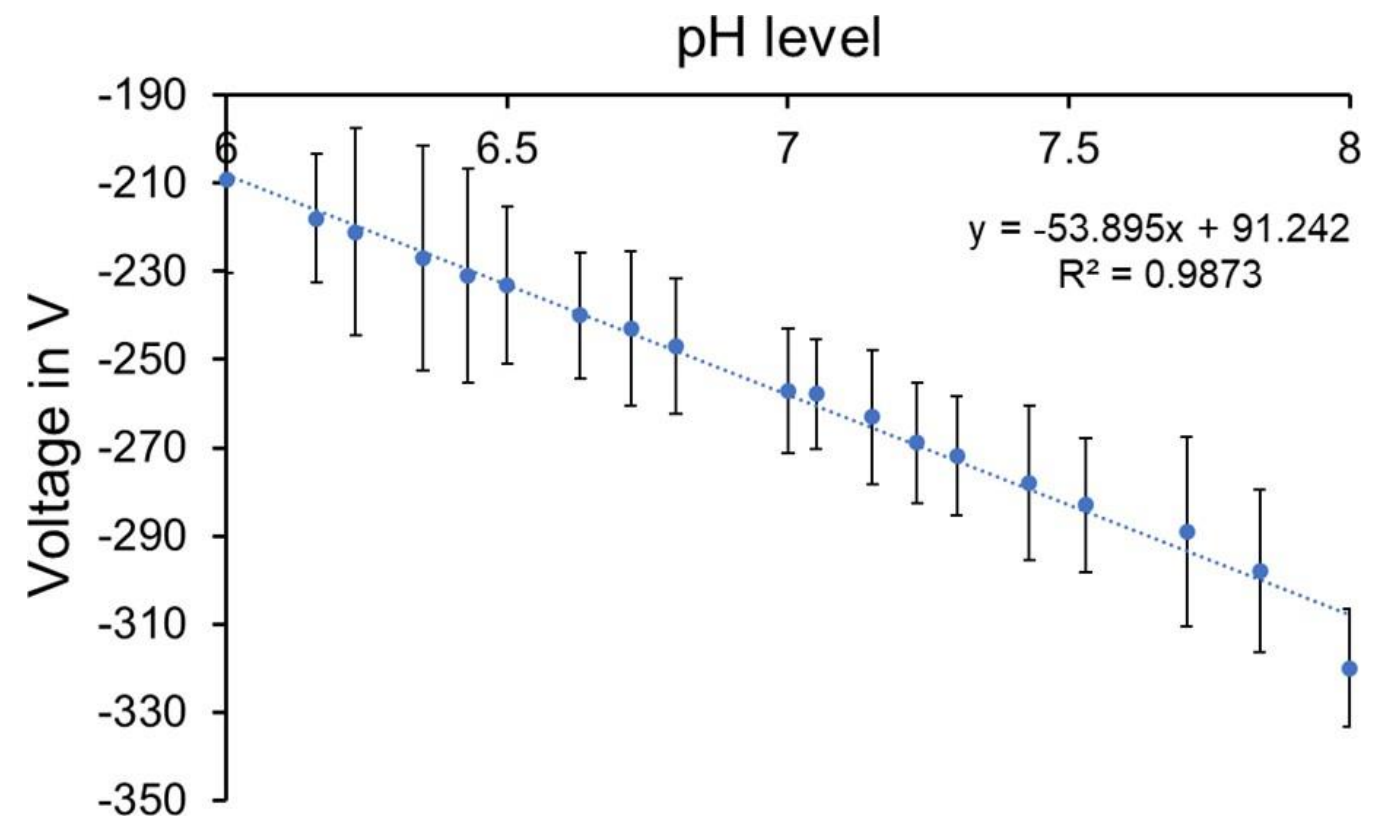

Fig. B.6. Calibration curve (pH6-pH8) 


\section{Appendix C: Examples of the measured data}

\section{C.1 Bovine Embryos Metabolism: Basal vs Glycolysis}

Examples of the measured current vs time of the metabolism of 5 embryos before and after adding oligomycin are shown in Figs. C.1 (a-c).

(a)
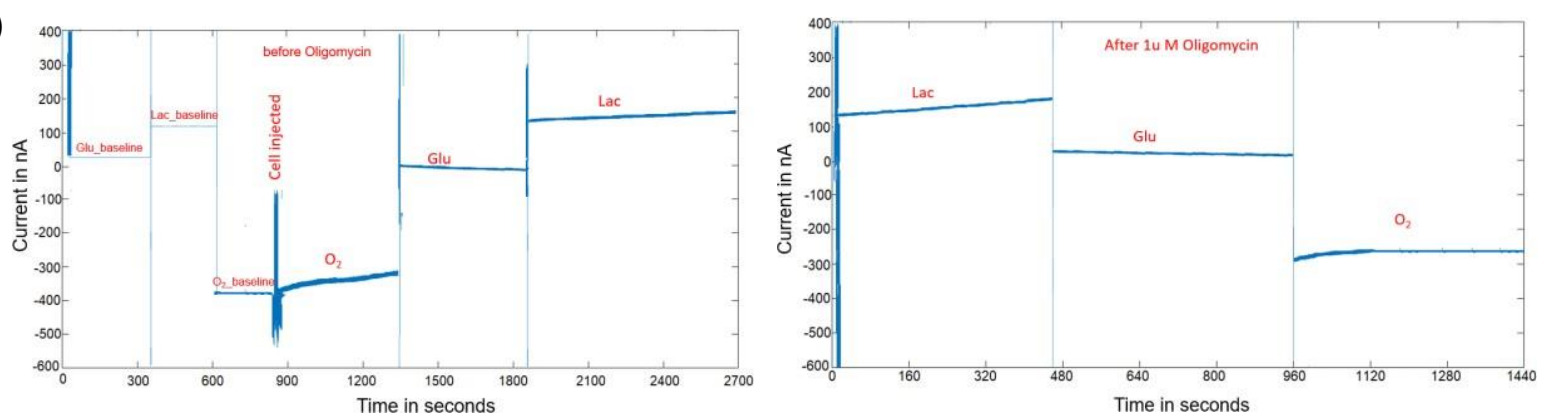

(b)
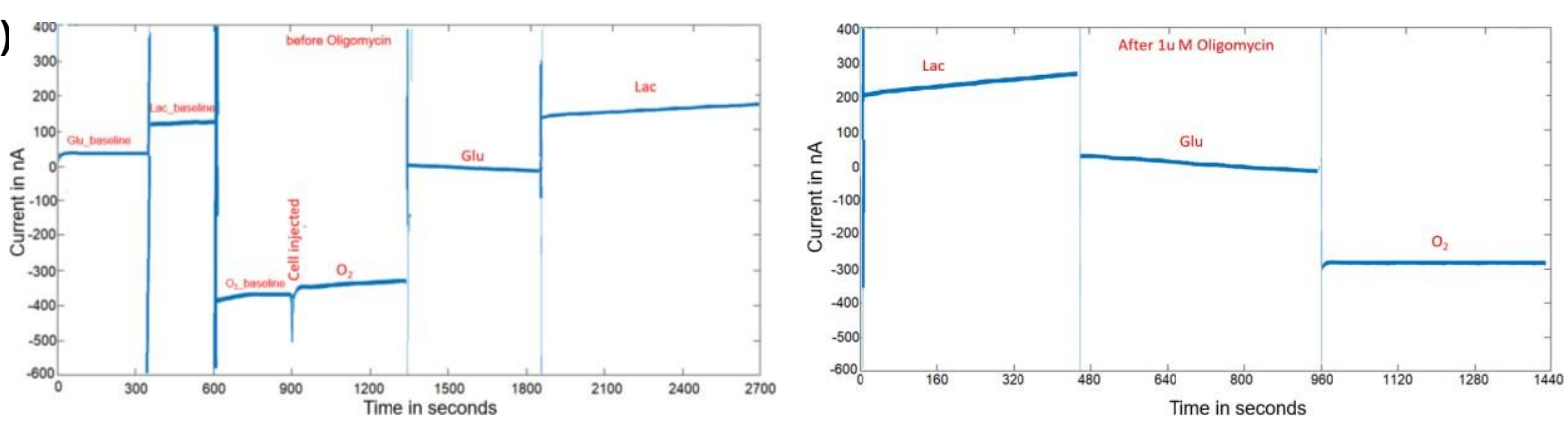

(c)
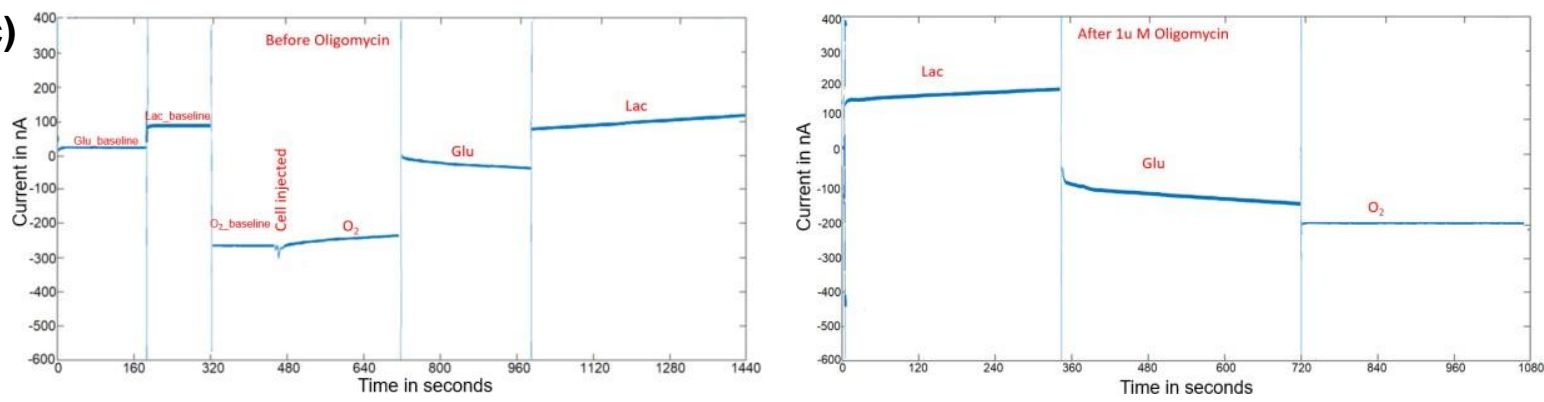

Fig. C.1. The currents readings $n A$ vs time for 5 different embryos: (a)-(c).

\section{C.2 Equine Embryos Metabolism (Basal, Oligomycin, FCCP ${ }_{1-3}$ )}

Fig. C.2 shows an example of the measured data. The figure to the left shows the oxygen sensor current readings at basal, oligomycin, and FCCP1 to FCCP3, and the figure to the right shows the glucose and lactate sensors current readings at same conditions. A baseline reading 
was taken for all sensors. The cell was injected into the well that has oxygen and $\mathrm{pH}$ and specifically on top of the oxygen sensor. the readings of oxygen, $\mathrm{pH}$, glucose and lactate was recorded under all conditions of basal, oligo, and FCCP1 to FCCP3. pH is measured using potenometric method (voltage not current) so that is why the readings of $\mathrm{pH}$ are not in the same figures of oxygen, glucose and lactate.
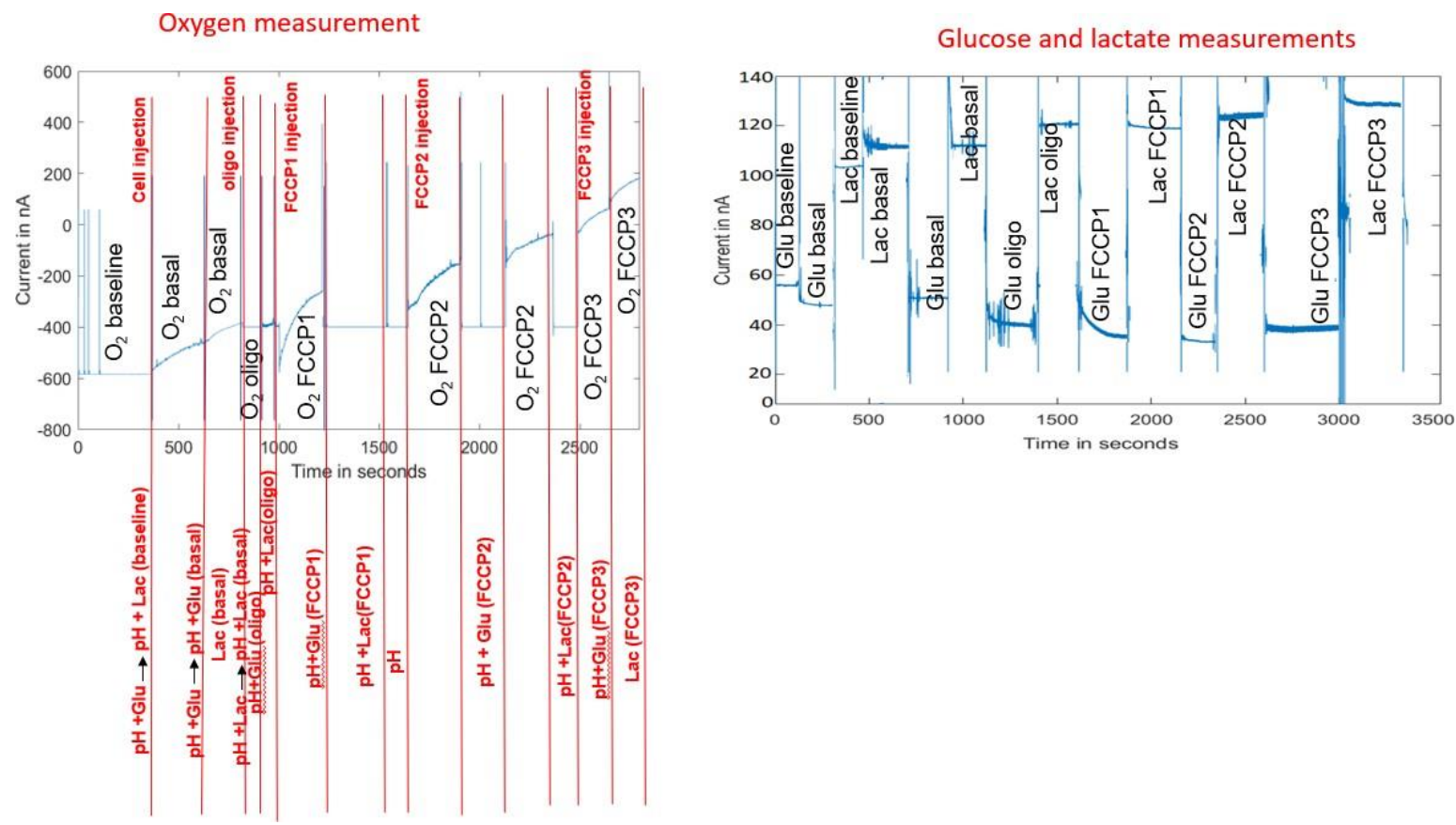

Fig. C.2. Example of equine embryo measured data 


\section{Appendix D: Interference Tests}

\section{D.1 Effect of Enzymes Reactions on $\mathrm{O}_{2}$ Level in Multi-Sensor Platform}

Considering the following equations from Chapter 2,

$\mathrm{L}-$ lactate $+\mathrm{O}_{2} \stackrel{\text { LOD }}{\longrightarrow}$ Pyruvate $+\mathrm{H}_{2} \mathrm{O}_{2}$

$\mathrm{H}_{2} \mathrm{O}_{2} \rightarrow \mathrm{O}_{2}+2 \mathrm{H}^{+}+2 \mathrm{e}^{-}$

Glucose $+\mathrm{O}_{2} \stackrel{\text { COD }}{\longrightarrow}$ gluconolactone $+\mathrm{H}_{2} \mathrm{O}_{2}$

$\mathrm{H}_{2} \mathrm{O}_{2} \rightarrow \mathrm{O}_{2}+2 \mathrm{H}^{+}+2 \mathrm{e}^{-}$

In each reaction glucose and lactate enzymes will need to take one oxygen molecule to produce $\mathrm{H}_{2} \mathrm{O}_{2}$, and this molecule will be produced again after $\mathrm{H}_{2} \mathrm{O}_{2}$ oxidation reaction happens.

However, to make sure that the oxygen molecules that are used for enzymatic reaction will not affect the oxygen consumption level, experiment was done on 6 embryos.

Oxygen consumption was measured in two chambers (Fig. 5.1, Chapter 5): one has enzymes on the working electrodes corresponding to glucose and lactate measurements, while the other chamber has no enzymes anywhere. Only oxygen consumption was measured for each cell, and the cell was moved from one chamber to another to compare the difference in oxygen readings.

- Test 1: cell was measured when no enzymes on, then was moved to a device with enzymes on.

- Test 2: cell was measured in the device with enzymes on, then was moved to a device with no enzymes.

The results have shown that there is no significant difference between the oxygen readings with or without enzymes and so no interference effect from the enzymes on the oxygen consumption level. Fig. D.1 shows a bar chart conclusion of the oxygen readings of 6 - cells in 
two chambers (with enzymes and without enzymes). The measured current vs time figures for 5 cells are shown in Fig. D.2 (A-E).

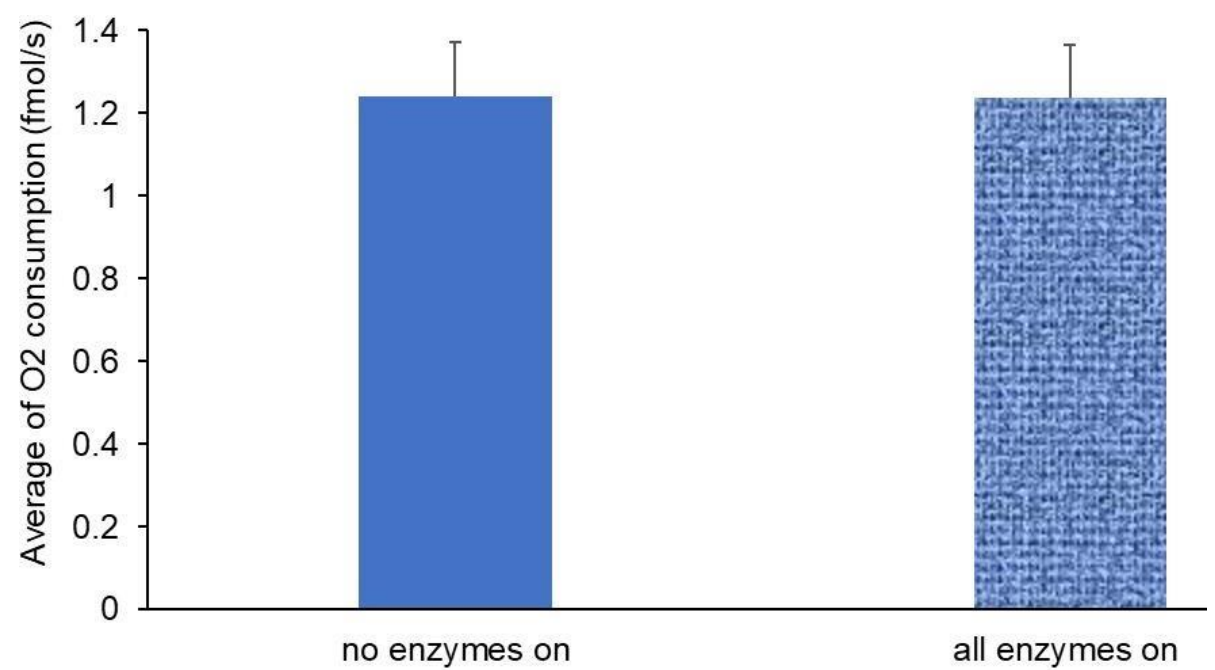

Fig. D.1. Oxygen consumption level of embryos each measured in two chambers: with and without enzymes on. Error bars are (SD) between 6 embryos.
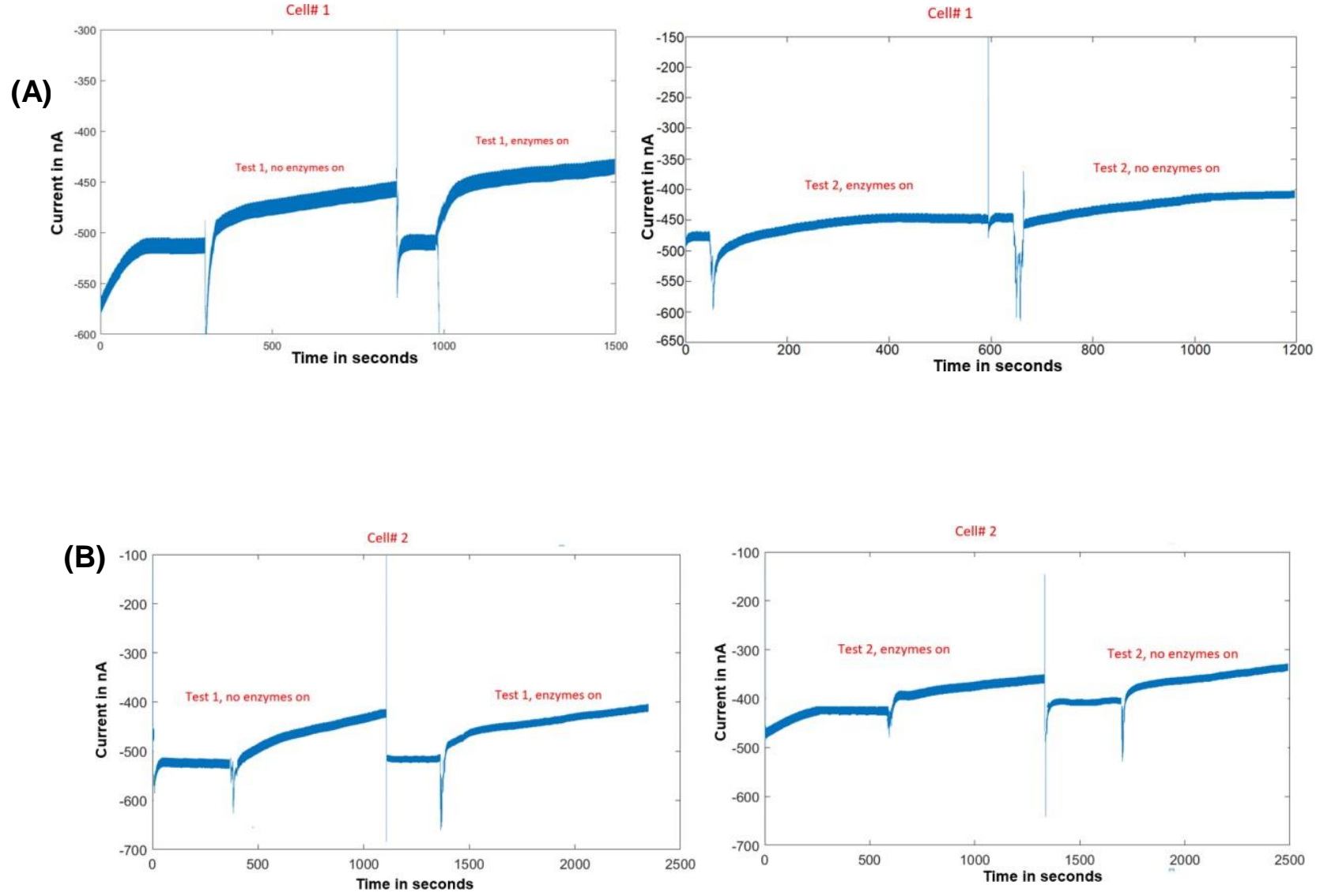
(C)
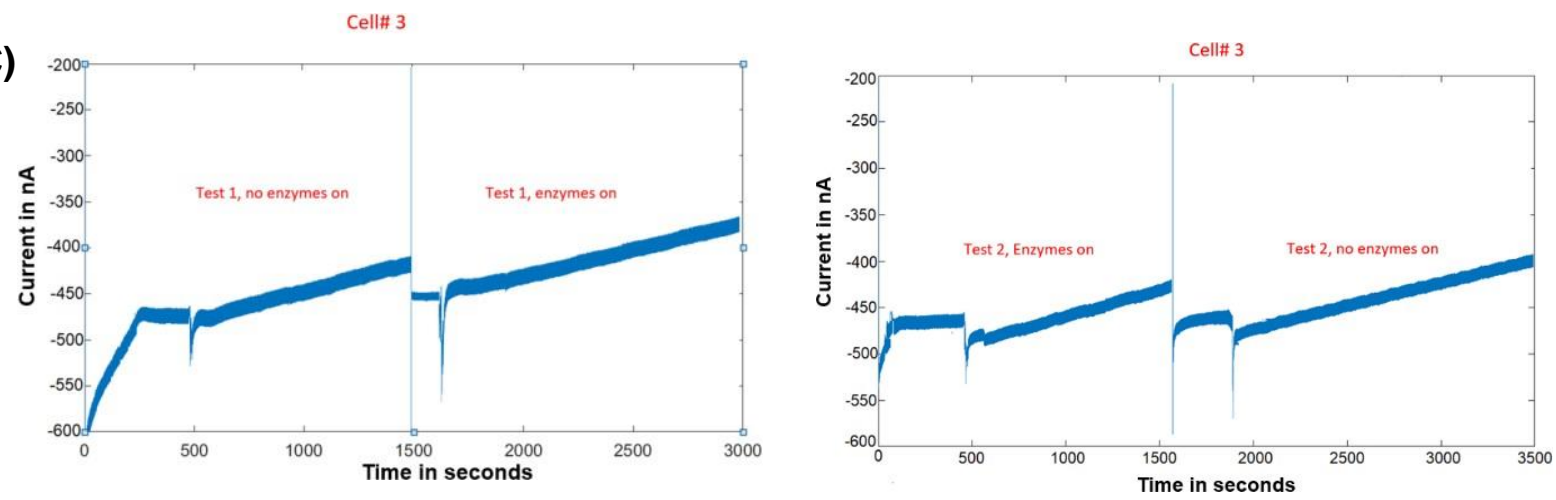

(D)

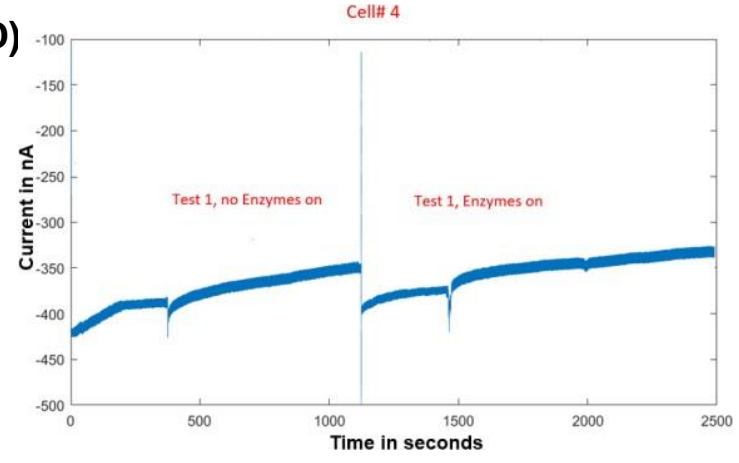

(E)

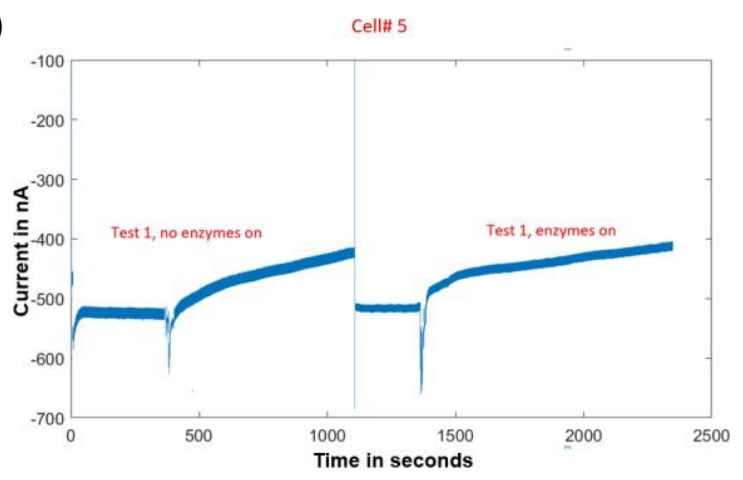

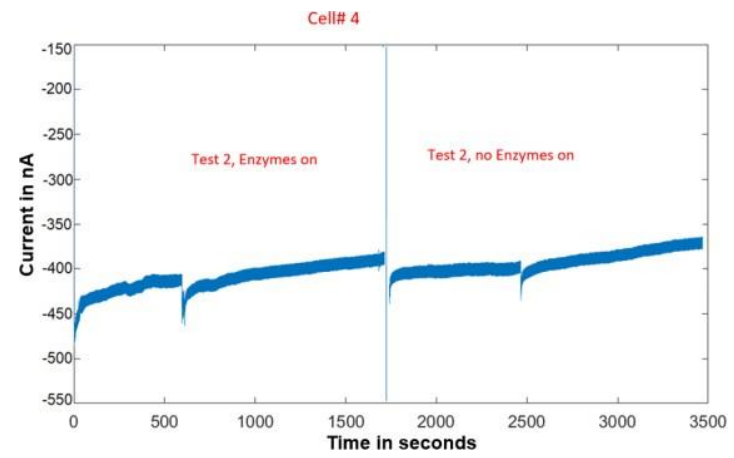

Cell\# 5

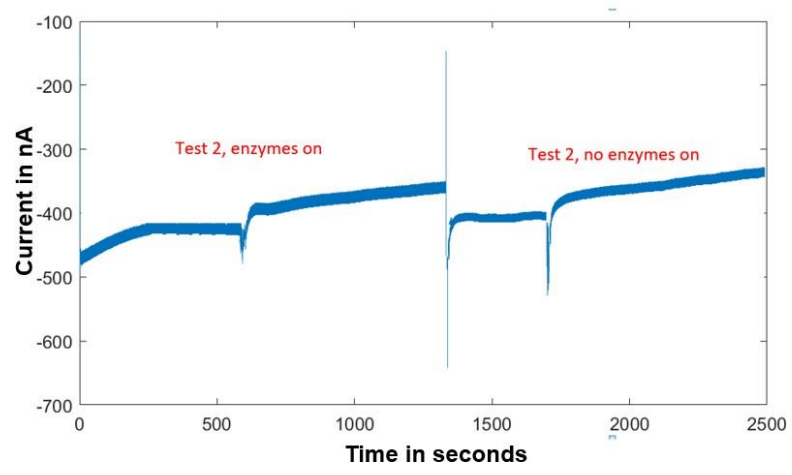

Fig. D.2. The currents readings nA vs time for 5 different embryos: (a)-(e).

\section{D.2 Interference between Enzyme Sensors in Multi-Sensor System}

The interference experiments were performed using a different sensor configuration with shorter distances between interfering sensors. Each well (Fig. D.3A) has six sensors that will ultimately be used for sensing of up to six different analytes. The multi-sensor design mask (Fig. D.3B) was e-beam patterned on an ITO coated glass, and the chip was fabricated using the 
similar steps described in Sections 6.2.2 and 6.2.3. The final multi-sensor chip after ITO etching and gold evaporation is shown in Fig. D.3C.

(A)

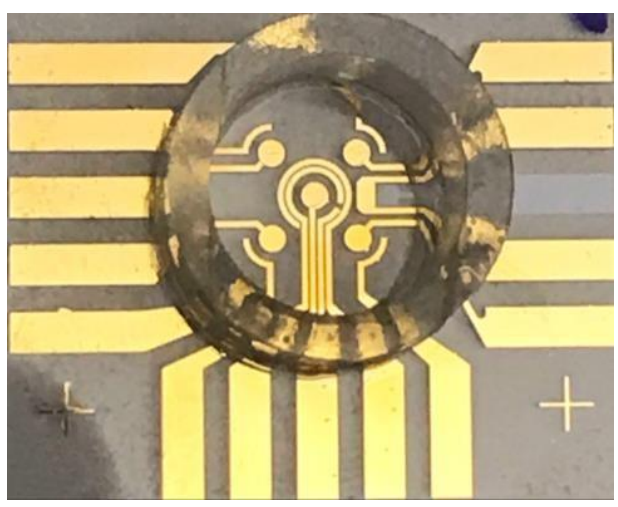

(B)

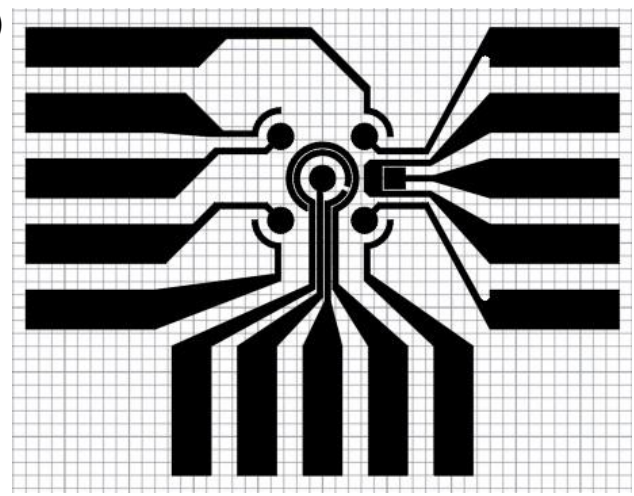

(C)

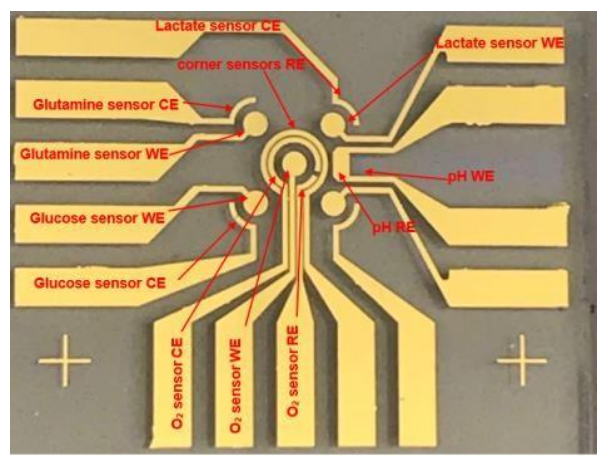

Fig. D.3. (A) Four wells design. (B) Single well chip. (C) The mask used for the design.

\section{D.2.1 Interference Raw Data Examples}

Figs. S.12A-S.12C show an example of the interference raw data, when glucose sensor was used as an intiating sensor and and the effect of glucose titration $(0.1 \mathrm{mM}, 0.5 \mathrm{mM}, 1 \mathrm{mM}$ and 2 $\mathrm{mM}$ ) was measured at two lactate sensors at different distances (sens3 at $4.6 \mathrm{~mm}$ and sens 4 at $3.2 \mathrm{~mm}$ ). 

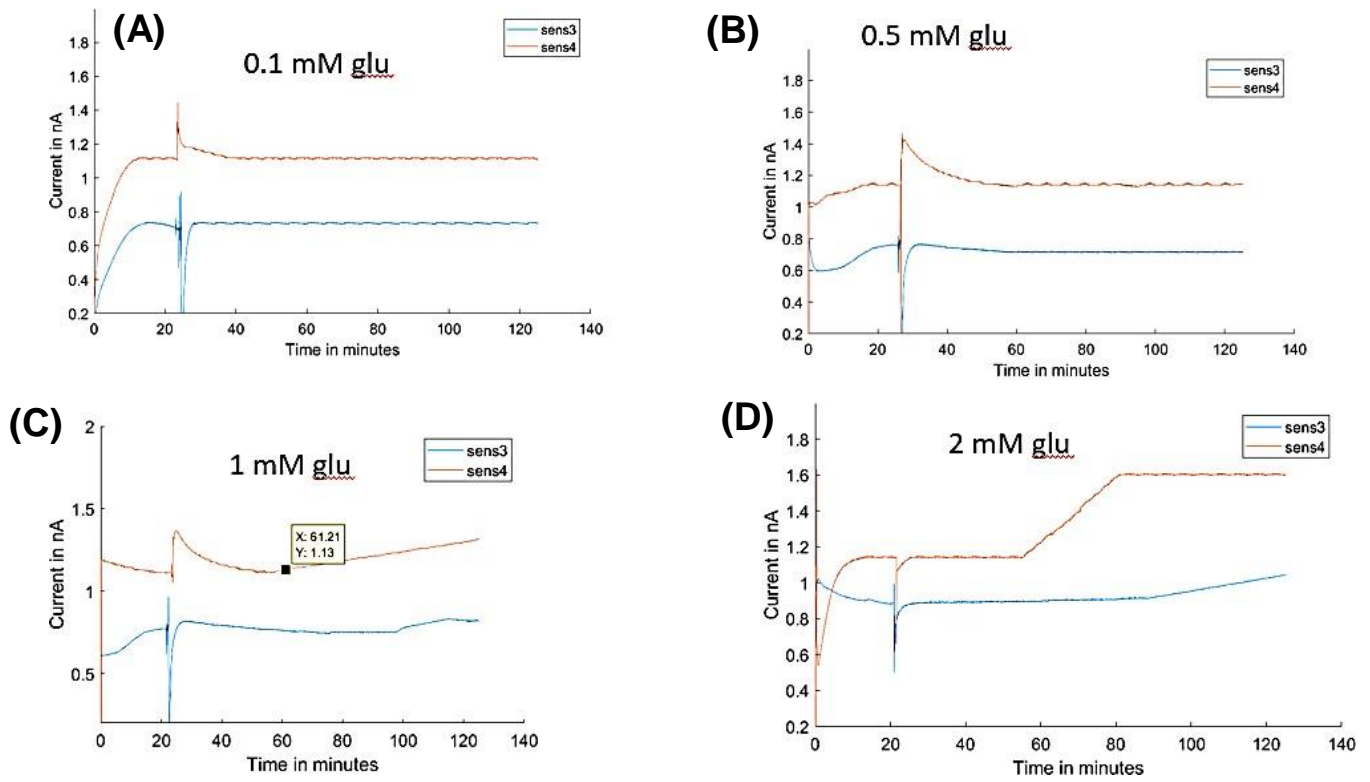

Fig. D.4. Effect of glucose titration $(0.1 \mathrm{mM}$ to $2 \mathrm{mM}),(\mathbf{A})$ to $(\mathbf{B})$ on lactate sensors at two different distances (sens3 at $4.6 \mathrm{~mm}$, and sens 4 at $3.2 \mathrm{~mm}$ ) 


\section{Appendix E: Effect of the platform on embryos development}

After some experiments, some cells were taken back to the culturing system to continue the development and that helped us to study the effect of the platform on the cell development in long term. However, the percentages of the cells from all stages that were developed until the last stage were $33 \%$, while $67 \%$ of the cells either died after 24 hours or couldn't reach the last stage of development. Fig. E.1 shows the bar-chart conclusion of analytes consumption/production for all developed samples from all stages.

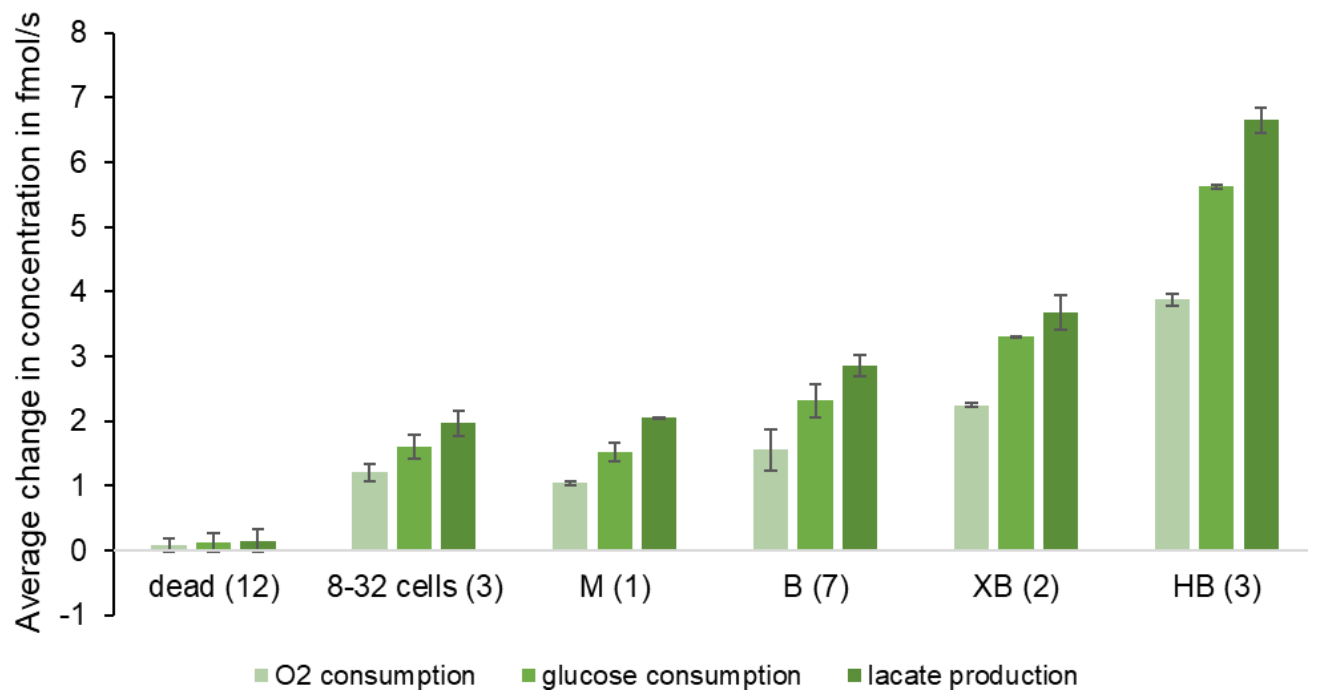

Fig. E.1. The analytes consumption/production of the samples that were developed to the final stage successfully (33\% of all cells).

The percentage of the cells developed from each stage is: $33 \%$ of $8-32$ cells, $25 \%$ of $M, 88 \%$ of $B$, $28 \%$ of $\mathrm{XB}$, and $75 \%$ of HB. Fig. E.2 (A-E) shows the bar-chart conclusion of analytes consumption/production for developed vs non-developed samples from each stage. 


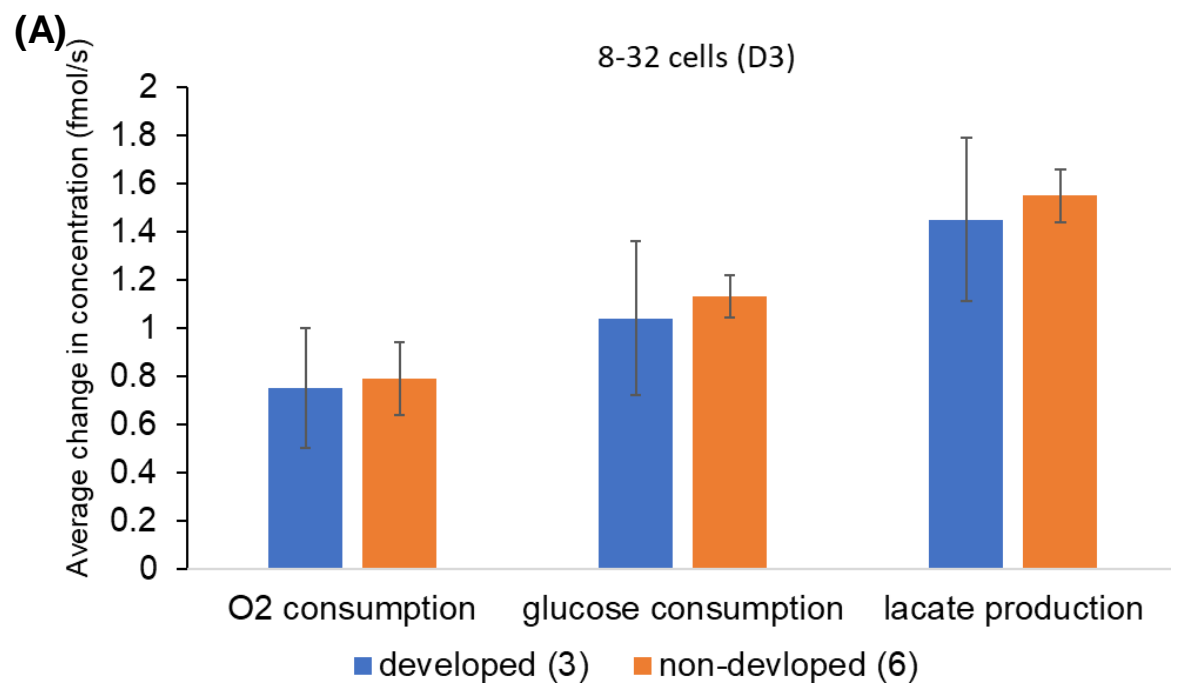

(B)

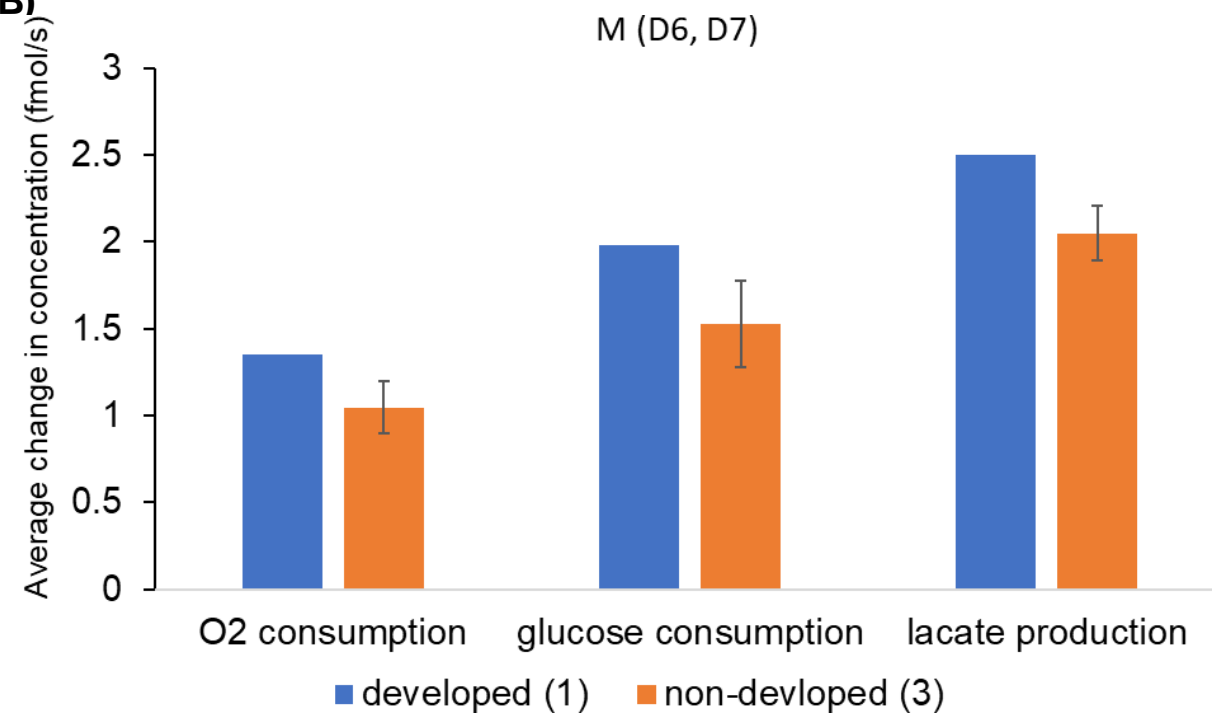

(C)

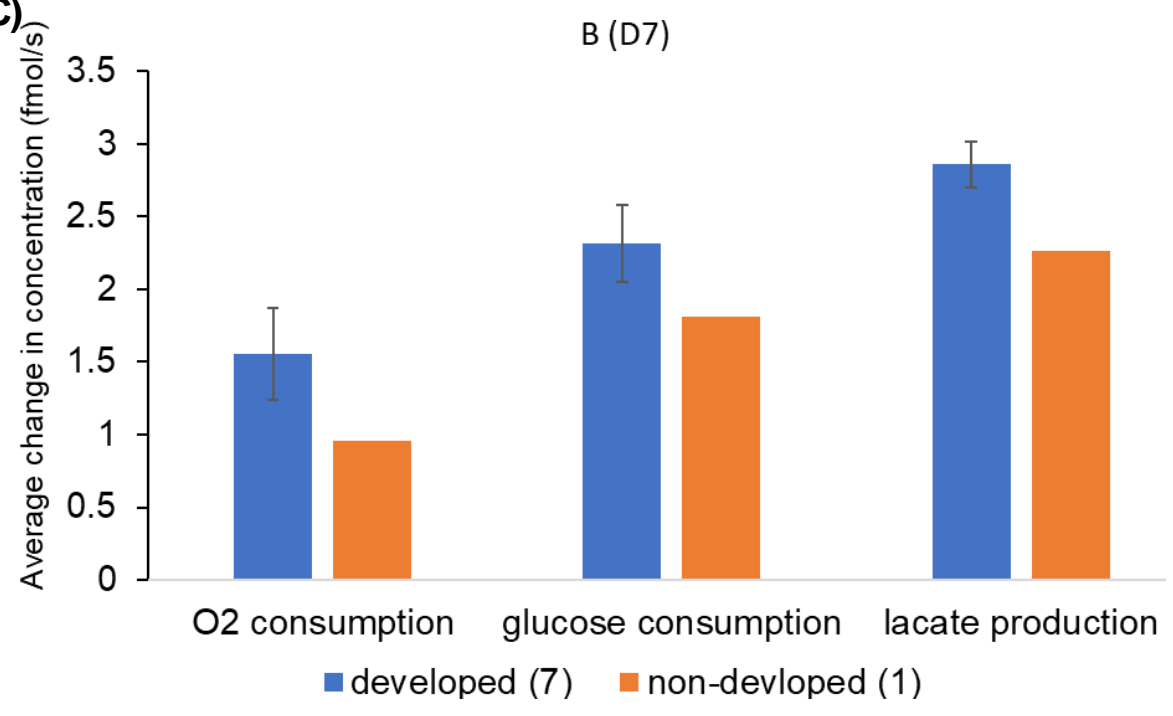




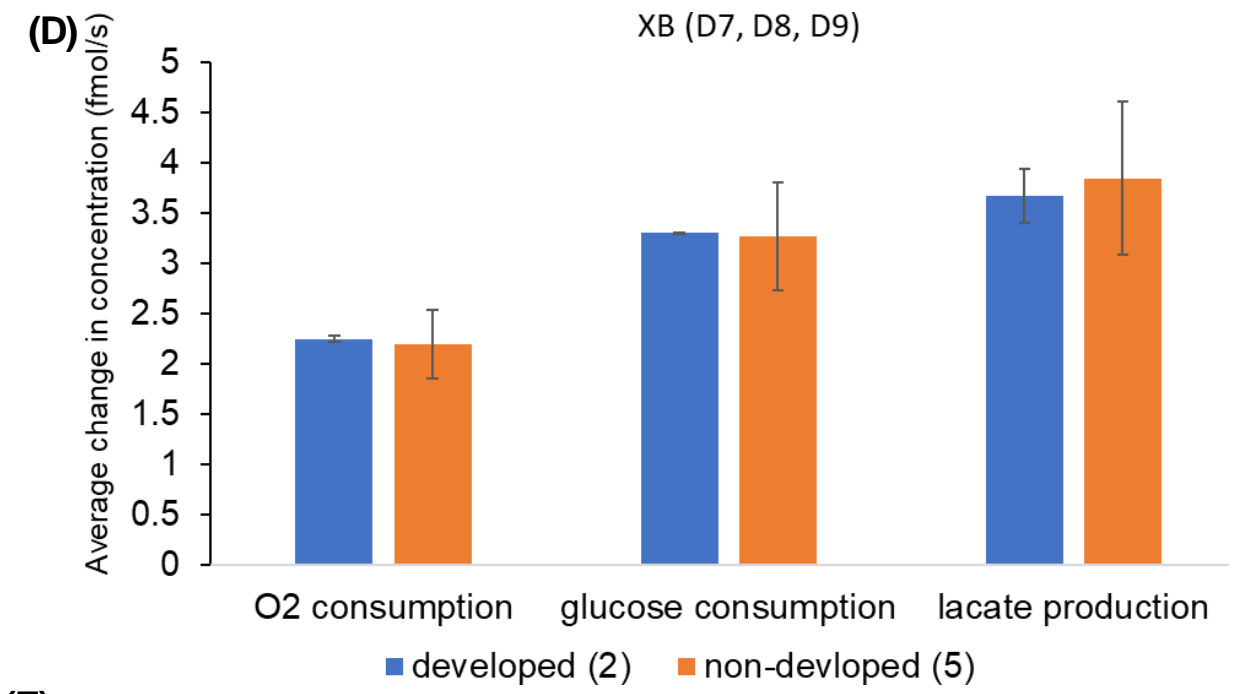

(E)

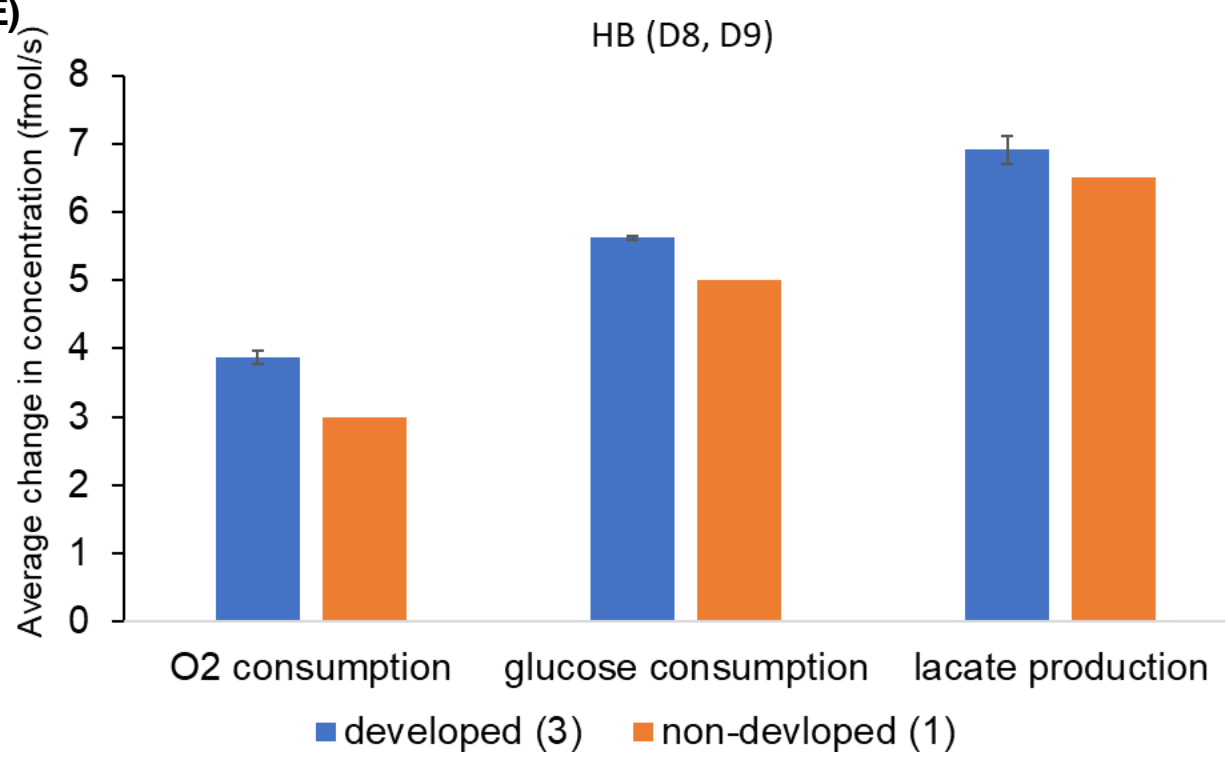

Fig. E.2. The analytes consumption/production of the devloped vs non-developed samples from all stages $(A)-(E)$. 


\section{Appendix F: Matlab Gui}

Matlab Gui was used for analyzing the measured data and converting them into consumption or production rates in fmol/s. I have included the code used for oxygen consumption rate and similar coding was used for lactate and glucose rates. The complete code is very long, so I only included the part of the code used for oxygen consumption rate under all conditions (basal, oligo, FCCP1, FCCP2, FCCP3).

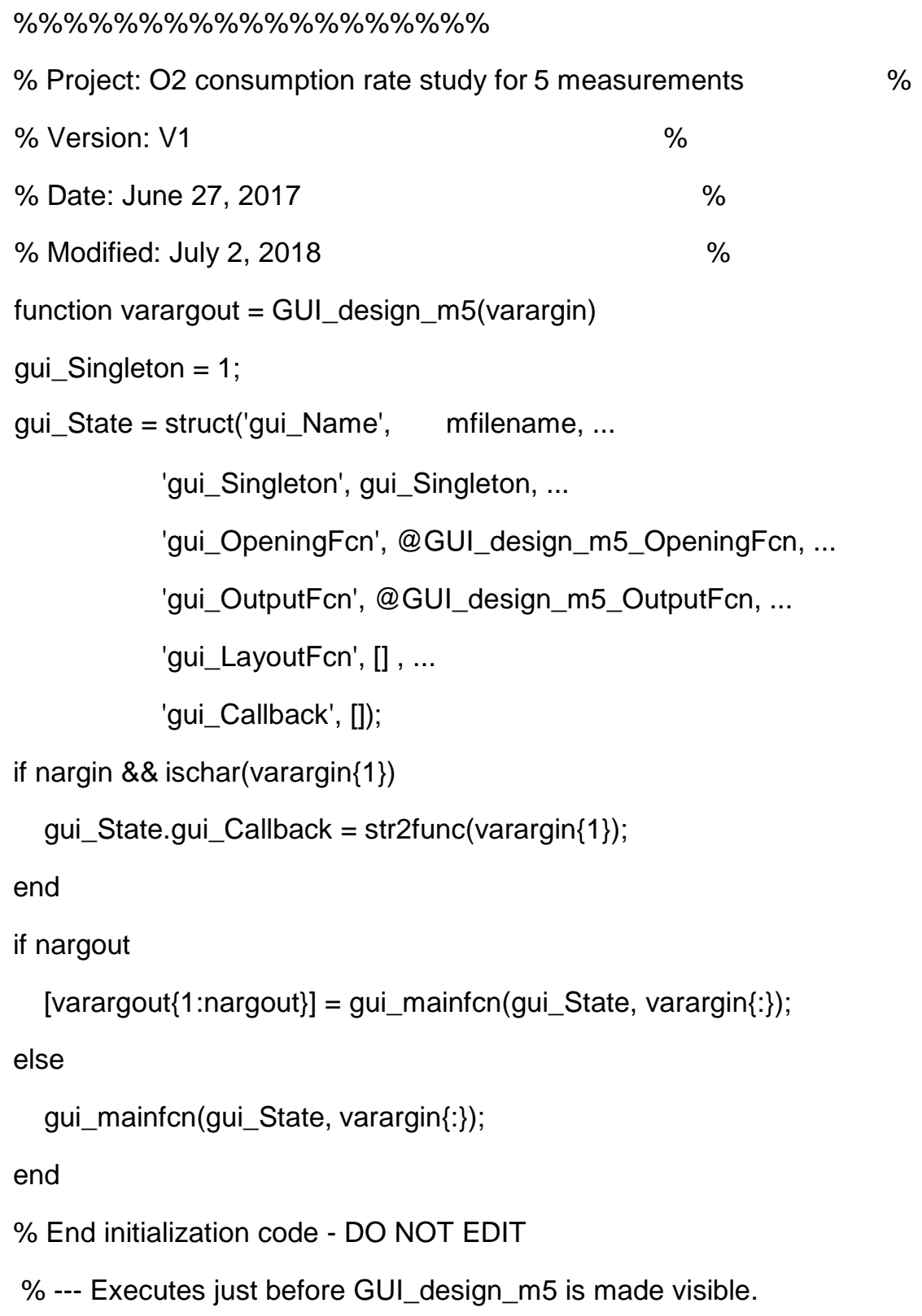


function GUI_design_m5_OpeningFcn(hObject, eventdata, handles, varargin)

$x \lim ([0$ 40])

$y \lim ([-2000-1])$

xlabel('time $\left.(\min )^{\prime}\right)$

ylabel('current $(n A)$ ')

title('Oxygen Consumption Measurement')

display('Current to Oxygen Consumption Rate Conversion')

display('Please setup the measurement.')

\% Choose default command line output for GUI_design_m5

handles.output $=$ hObject;

$\%$ Update handles structure

guidata(hObject, handles);

$\%$--- Executes on button press in load_button.

function load_button_Callback(hObject, eventdata, handles)

global data unsmoothed data2 cell_height\% data2 filter_fn

[file_reading,path] = uigetfile('*.txt');

selectedfile = fullfile(path,file_reading);

set(handles.Sample_ID,'String', file_reading);

raw = load(selectedfile);

$\% \% \%$ Since it is Oxygen measurement, any data points greater than zero \%\%\%

$\% \% \%$ is not consodered valid. Therefore I force it to be zero. $\% \%$

for $\mathrm{i}=1$ :length(raw)

if $\operatorname{raw}(\mathrm{i})<0$

$\operatorname{raw}(\mathrm{i})=\operatorname{raw}(\mathrm{i})$;

else

$\operatorname{raw}(\mathrm{i})=0$;

end

end

$\% \% \% \% \% \% \%$ find the non-zero parts of the data, and merge the data $\% \% \% \% \% \% \% \% \% \%$

$B=$ find $(r a w)$;

for $\mathrm{i}=1$ :length(B)

unsmoothed $(i)=\operatorname{raw}(B(i))$; 
end

\%\%\%\%\%\%\%\%\%\%\%\%\%\%\%\%\%\%\%\%\%\%\%\%\%\%\%\%\%\%\%\%\%\%\%\%\%\%\%\%\%\%\%\%\%\%\%\%\%\%\%\%

cell_height $=$ str2double $(g e t($ handles.cell_height, 'string'));

data2 = 0 ;

data $=$ unsmoothed;

plot(linspace $(0$, length(unsmoothed)/60/40,length(unsmoothed))',unsmoothed...

,'LineWidth',1.5)

grid on

xlabel('Time $(\min )$ ')

ylabel('Current $\left.(\mathrm{nA}))^{\prime}\right)$

title('Oxygen Consumption Measurement')

display('Data Loaded Successfully...')

function basal_s_Callback(hObject, eventdata, handles)

$\%$ hObject handle to basal_s (see GCBO)

$\%$ eventdata reserved - to be defined in a future version of MATLAB

$\%$ handles structure with handles and user data (see GUIDATA)

\% Hints: get(hObject,'String') returns contents of basal_s as text

$\% \quad$ str2double(get(hObject,'String')) returns contents of basal_s as a double

$\%$--- Executes during object creation, after setting all properties.

function basal_s_CreateFcn(hObject, eventdata, handles)

\% hObject handle to basal_s (see GCBO)

$\%$ eventdata reserved - to be defined in a future version of MATLAB

$\%$ handles empty - handles not created until after all CreateFcns called

$\%$ Hint: edit controls usually have a white background on Windows.

$\% \quad$ See ISPC and COMPUTER.

if ispc \&\& isequal(get(hObject,'BackgroundColor'), get(0,'defaultUicontrolBackgroundColor')) set(hObject,'BackgroundColor','white');

end

function basal_f_Callback(hObject, eventdata, handles)

$\%$ hObject handle to basal_f (see GCBO)

$\%$ eventdata reserved - to be defined in a future version of MATLAB

$\%$ handles structure with handles and user data (see GUIDATA) 
\% Hints: get(hObject,'String') returns contents of basal_f as text

$\% \quad$ str2double(get(hObject,'String')) returns contents of basal_f as a double

$\%$--- Executes during object creation, after setting all properties.

function basal_f_CreateFcn(hObject, eventdata, handles)

$\%$ hObject handle to basal_f (see GCBO)

$\%$ eventdata reserved - to be defined in a future version of MATLAB

$\%$ handles empty - handles not created until after all CreateFcns called

$\%$ Hint: edit controls usually have a white background on Windows.

$\% \quad$ See ISPC and COMPUTER.

if ispc \&\& isequal(get(hObject,'BackgroundColor'), get(0,'defaultUicontrolBackgroundColor')) set(hObject,'BackgroundColor','white');

end

$\%$--- Executes on button press in basal_run.

function basal_run_Callback(hObject, eventdata, handles)

global data data2 unsmoothed cell_height

if data2 $==0$

plot(linspace $(0$, length(data) $/ 60 / 40$,length(data))',data...

,'LineWidth',1.5)

else

plot(linspace(0,length(data2)/60/40,length(data2))',...

data2,'LineWidth',1.5)

hold on

plot(linspace(0,length(data2)/60/40,length(data2))',data)

end

grid on

xlabel('Time (min)')

ylabel('Current (nA)')

title('Oxygen Consumption Measurement')

basal_start =str2double(get(handles.basal_s, 'string'));

basal_stop $=$ str2double(get(handles.basal_f,'string'));

hold off 


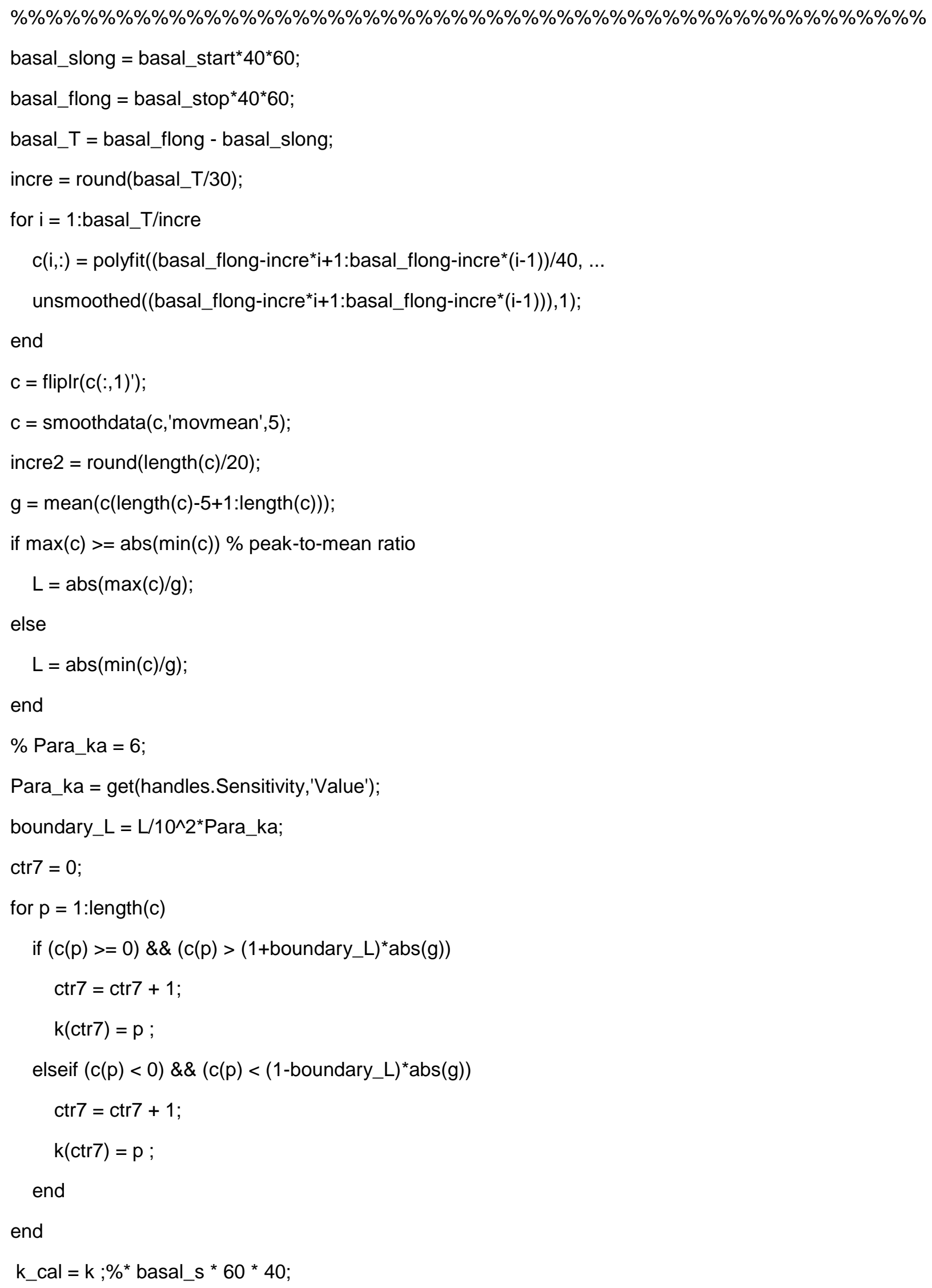




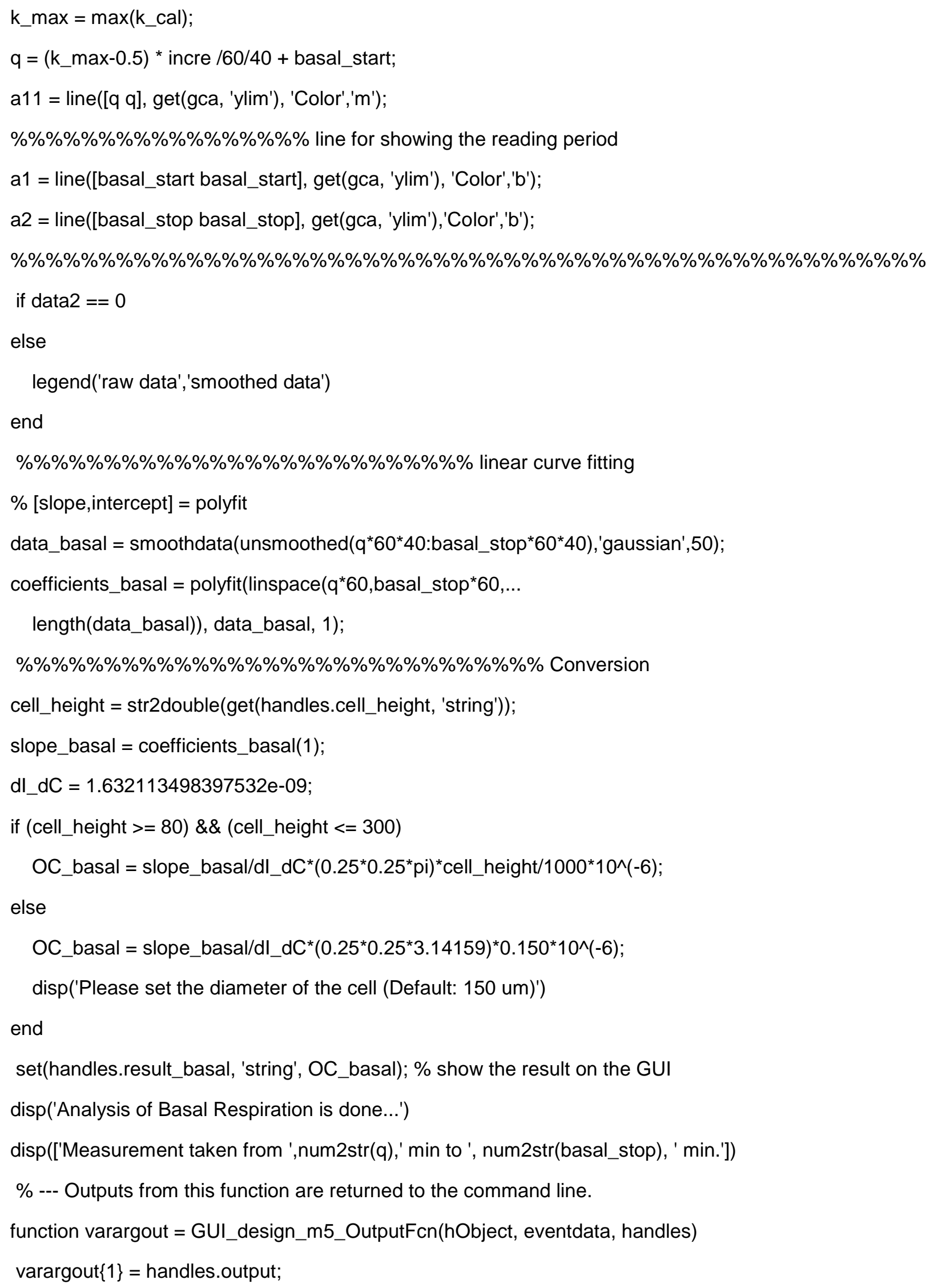




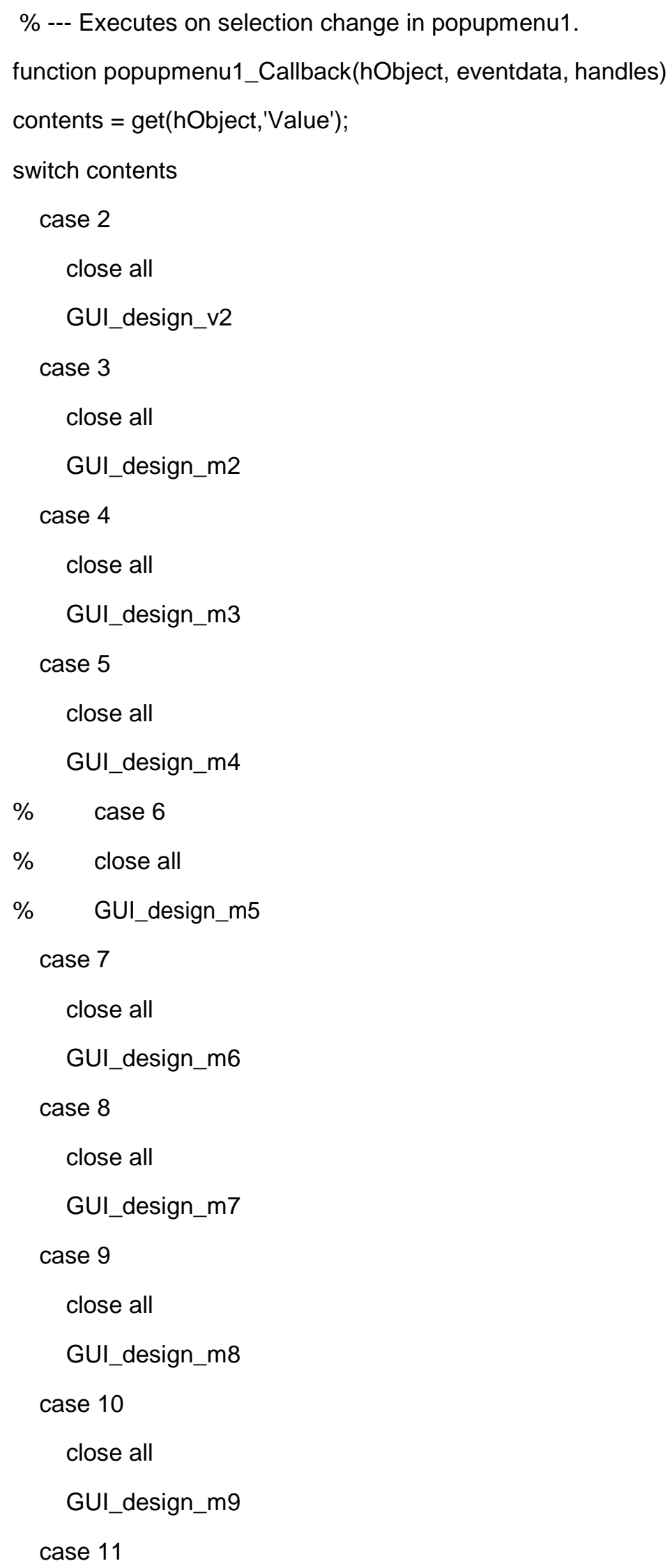


close all

GUI_design_m10

case 12

close all

GUI_design_m11

case 13

close all

GUI_design_m12

case 14

close all

GUI_design_m13

case 15

close all

GUI_design_m14

case 16

close all

GUI_design_m15

otherwise

end

$\%$--- Executes during object creation, after setting all properties.

function popupmenu1_CreateFcn(hObject, eventdata, handles)

if ispc \&\& isequal(get(hObject,'BackgroundColor'), get(0,'defaultUicontrolBackgroundColor'))

set(hObject,'BackgroundColor','white');

end

function m2_s_Callback(hObject, eventdata, handles)

$\%$ hObject handle to m2_s (see GCBO)

$\%$ eventdata reserved - to be defined in a future version of MATLAB

$\%$ handles structure with handles and user data (see GUIDATA)

\% Hints: get(hObject,'String') returns contents of m2_s as text

$\% \quad$ str2double(get(hObject,'String')) returns contents of m2_s as a double

$\%$--- Executes during object creation, after setting all properties.

function m2_s_CreateFcn(hObject, eventdata, handles) 
$\%$ hObject handle to m2_s (see GCBO)

$\%$ eventdata reserved - to be defined in a future version of MATLAB

$\%$ handles empty - handles not created until after all CreateFcns called

$\%$ Hint: edit controls usually have a white background on Windows.

$\% \quad$ See ISPC and COMPUTER.

if ispc \&\& isequal(get(hObject,'BackgroundColor'), get(0,'defaultUicontrolBackgroundColor')) set(hObject,'BackgroundColor','white');

end

function m2_f_Callback(hObject, eventdata, handles)

$\%$ hObject handle to $\mathrm{m}_{2} \_$(

$\%$ eventdata reserved - to be defined in a future version of MATLAB

$\%$ handles structure with handles and user data (see GUIDATA)

$\%$ Hints: get(hObject,'String') returns contents of m2 f as text

$\% \quad$ str2double(get(hObject,'String')) returns contents of $m 2 \_f$ as a double

$\%$--- Executes during object creation, after setting all properties.

function m2_f_CreateFcn(hObject, eventdata, handles)

$\%$ hObject handle to $m 2$ f (see GCBO)

$\%$ eventdata reserved - to be defined in a future version of MATLAB

$\%$ handles empty - handles not created until after all CreateFcns called

$\%$ Hint: edit controls usually have a white background on Windows.

$\% \quad$ See ISPC and COMPUTER.

if ispc \&\& isequal(get(hObject,'BackgroundColor'), get(0,'defaultUicontrolBackgroundColor')) set(hObject,'BackgroundColor','white');

end

$\%$--- Executes on button press in m2_run.

function m2_run_Callback(hObject, eventdata, handles)

global data data2 unsmoothed cell_height

if data2 $==0$

plot(linspace $(0$,length(data) $/ 60 / 40$,length(data))',data...

,'LineWidth',1.5)

else

plot(linspace(0,length(data2)/60/40,length(data2))',... 
data2,'LineWidth',1.5)

hold on

$\operatorname{plot}($ linspace $(0$, length(data2)/60/40,length(data2))',data)

end

grid on

xlabel('Time (min)')

ylabel('Current (nA)')

title('Oxygen Consumption Measurement')

m2_start = str2double(get(handles.m2_s, 'string'));

m2_stop = str2double(get(handles.m2_f,'string'));

hold off

\%\%\%\%\%\%\%\%\%\%\%\%\%\%\%\%\%\%\%\%\%

$\mathrm{m} 2$ _slong $=\mathrm{m} 2$ _start ${ }^{*} 40^{*} 60$;

m2_flong $=$ m2_stop $* 40 * 60$

$\mathrm{m} 2 \_\mathrm{T}=\mathrm{m} 2$ _flong - m2_slong;

incre $=$ round $\left(\mathrm{m} 2 \_\mathrm{T} / 30\right)$;

for $\mathrm{i}=1: \mathrm{m} 2 \_$T/incre

$c(i,:)=$ polyfit $\left(\left(m 2\right.\right.$ flong-incre ${ }^{*} i+1: m 2$ flong-incre $\left.*(i-1)\right) / 40, \ldots$

unsmoothed $\left(\left(m 2\right.\right.$ flong-incre ${ }^{*} i+1: m 2$ flong-incre $\left.\left.\left.{ }^{*}(i-1)\right)\right), 1\right)$;

end

$C=f \operatorname{liplr}\left(c(:, 1)^{\prime}\right)$;

$\mathrm{c}=$ smoothdata $(\mathrm{c}$, 'movmean',5);

incre2 = round(length(c)/20);

$g=$ mean $(c($ length $(c)-5+1$ :length $(c)))$;

if $\max (c)>=\operatorname{abs}(\min (c)) \%$ peak-to-mean ratio

$\mathrm{L}=\operatorname{abs}(\max (\mathrm{c}) / \mathrm{g})$;

else

$\mathrm{L}=\operatorname{abs}(\min (\mathrm{c}) / \mathrm{g}) ;$

end

Para_ka $=6$;

boundary_L = L/10^2*Para_ka;

$\operatorname{ctr} 7=0$; 


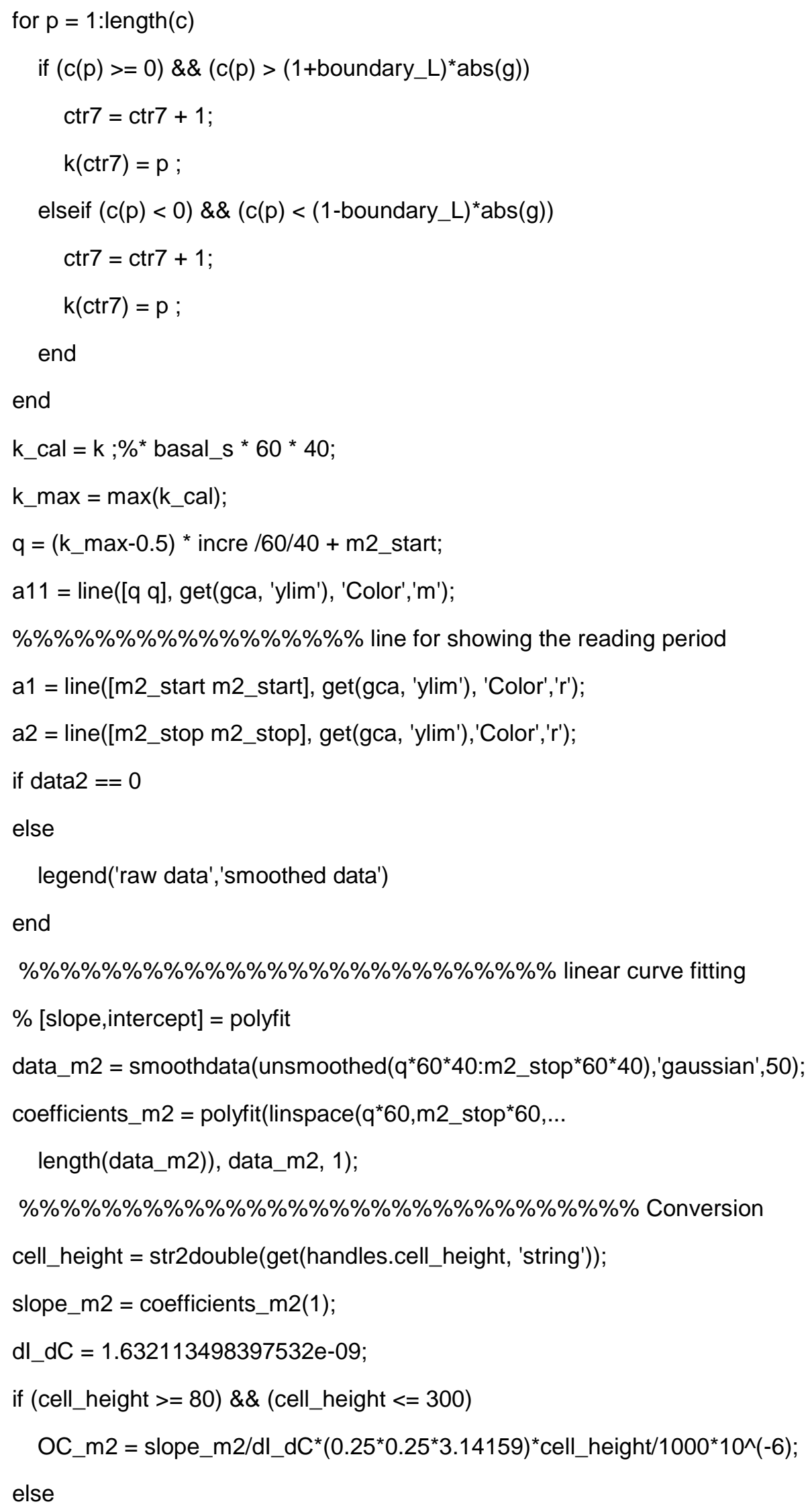


OC_m2 $=$ slope_m2/dl_dC ${ }^{\star}\left(0.25^{\star} 0.25^{\star} 3.14159\right)^{*} 0.150^{\star} 10^{\wedge}(-6)$;

disp('Please set the diameter of the cell (Default: $150 \mathrm{um}$ )')

end

set(handles.m2_result, 'string', OC_m2); \% show the result on the GUI

disp('Analysis of Measurement 2 is done...')

disp(['Measurement taken from ',num2str(q),' min to ', num2str(m2_stop), ' min.'])

function m3_s_Callback(hObject, eventdata, handles)

$\%$ hObject handle to m3_s (see GCBO)

$\%$ eventdata reserved - to be defined in a future version of MATLAB

$\%$ handles structure with handles and user data (see GUIDATA)

$\%$ Hints: get(hObject,'String') returns contents of m3_s as text

$\% \quad$ str2double(get(hObject,'String')) returns contents of m3_s as a double

$\%$--- Executes during object creation, after setting all properties.

function m3_s_CreateFcn(hObject, eventdata, handles)

$\%$ hObject handle to m3_s (see GCBO)

$\%$ eventdata reserved - to be defined in a future version of MATLAB

$\%$ handles empty - handles not created until after all CreateFcns called

$\%$ Hint: edit controls usually have a white background on Windows.

$\% \quad$ See ISPC and COMPUTER.

if ispc \&\& isequal(get(hObject,'BackgroundColor'), get(0,'defaultUicontrolBackgroundColor'))

set(hObject,'BackgroundColor','white');

end

function m3_f_Callback(hObject, eventdata, handles)

$\%$ hObject handle to m_f (see GCBO)

$\%$ eventdata reserved - to be defined in a future version of MATLAB

$\%$ handles structure with handles and user data (see GUIDATA)

\% Hints: get(hObject,'String') returns contents of m3_f as text

$\% \quad$ str2double(get(hObject,'String')) returns contents of m3_f as a double

$\%$--- Executes during object creation, after setting all properties.

function m3_f_CreateFcn(hObject, eventdata, handles)

$\%$ hObject handle to m_f (see GCBO) 
$\%$ eventdata reserved - to be defined in a future version of MATLAB

$\%$ handles empty - handles not created until after all CreateFcns called

$\%$ Hint: edit controls usually have a white background on Windows.

$\%$ See ISPC and COMPUTER.

if ispc \&\& isequal(get(hObject,'BackgroundColor'), get(0,'defaultUicontrolBackgroundColor')) set(hObject,'BackgroundColor','white');

end

$\%$--- Executes on button press in .

function m3_run_Callback(hObject, eventdata, handles)

global data data2 unsmoothed cell_height

if data2 $==0$

plot(linspace $(0$, length(data) $/ 60 / 40$,length(data))',data...

,'LineWidth',1.5)

else

plot(linspace(0,length(data2)/60/40,length(data2))',...

data2,'LineWidth',1.5)

hold on

plot(linspace(0,length(data2)/60/40,length(data2))',data)

end

grid on

xlabel('Time (min)')

ylabel('Current (nA)')

title('Oxygen Consumption Measurement')

m3_start =str2double (get(handles.m3_s, 'string'));

m3_stop $=$ str2double $($ get $($ handles.m3_f,'string') $)$;

hold off

m3_slong $=$ m3_start ${ }^{*} 40 * 60$;

m3_flong $=$ m3_stop $40 * 60$;

m3_T = m3_flong - m3_slong;

incre $=$ round $\left(\mathrm{m} 3 \_\mathrm{T} / 30\right)$;

for $\mathrm{i}=1$ :m3_T/incre

$c(i,:)=$ polyfit $\left(\left(m 3\right.\right.$ flong-incre ${ }^{*} i+1: m 3 \_f l o n g-$ incre $\left.^{*}(i-1)\right) / 40, \ldots$ 
unsmoothed $\left(\left(m 3\right.\right.$ flong-incre ${ }^{*}+1$ :m3_flong-incre $\left.\left.\left.{ }^{*}(i-1)\right)\right), 1\right)$;

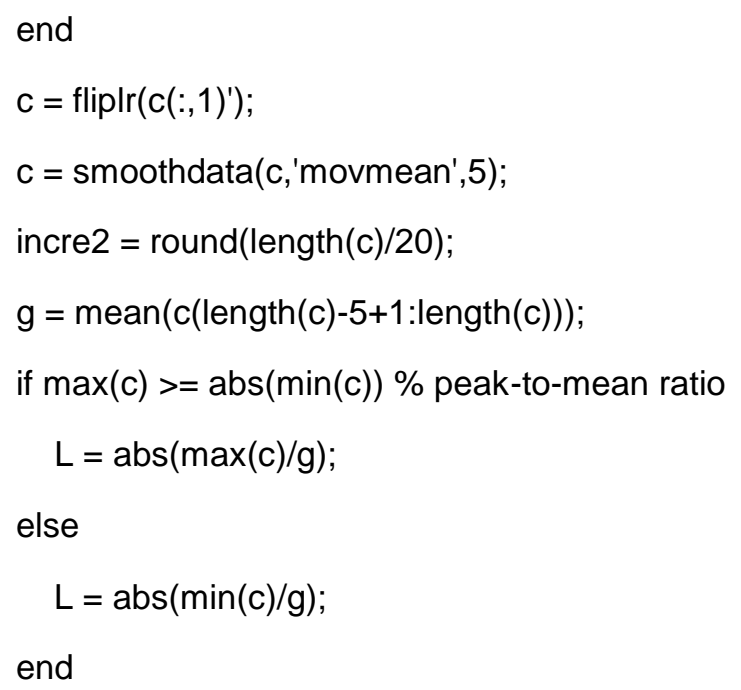




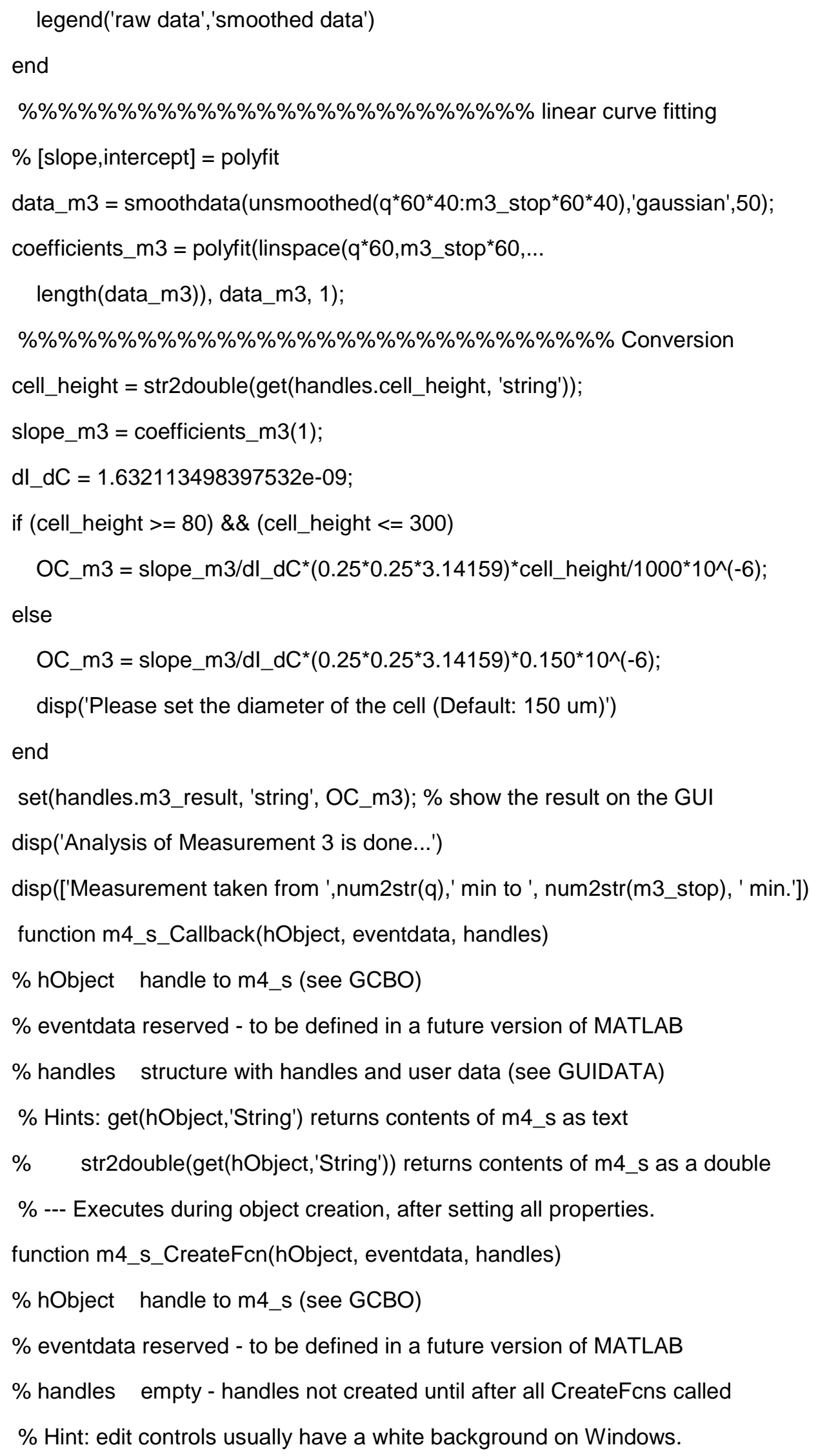


$\% \quad$ See ISPC and COMPUTER.

if ispc \&\& isequal(get(hObject,'BackgroundColor'), get(0,'defaultUicontrolBackgroundColor')) set(hObject,'BackgroundColor','white');

end

function m4_f_Callback(hObject, eventdata, handles)

$\%$ hObject handle to $\mathrm{m} 4$ _f (see GCBO)

$\%$ eventdata reserved - to be defined in a future version of MATLAB

$\%$ handles structure with handles and user data (see GUIDATA)

$\%$ Hints: get(hObject,'String') returns contents of m4_f as text

$\% \quad$ str2double(get(hObject,'String')) returns contents of m4_f as a double

$\%$--- Executes during object creation, after setting all properties.

function m4_f_CreateFcn(hObject, eventdata, handles)

$\%$ hObject handle to m4_f (see GCBO)

$\%$ eventdata reserved - to be defined in a future version of MATLAB

$\%$ handles empty - handles not created until after all CreateFcns called

$\%$ Hint: edit controls usually have a white background on Windows.

$\%$ See ISPC and COMPUTER.

if ispc \&\& isequal(get(hObject,'BackgroundColor'), get(0,'defaultUicontrolBackgroundColor')) set(hObject,'BackgroundColor','white');

end

$\%$--- Executes on button press in m4_run.

function m4_run_Callback(hObject, eventdata, handles)

global data data2 unsmoothed cell_height

if data2 $==0$

plot(linspace $(0$,length(data) $/ 60 / 40$,length(data))',data...

,'LineWidth',1.5)

else

plot(linspace(0,length(data2)/60/40, length(data2))',...

data2,'LineWidth',1.5)

hold on

plot(linspace(0,length(data2)/60/40,length(data2))',data) end

grid on 


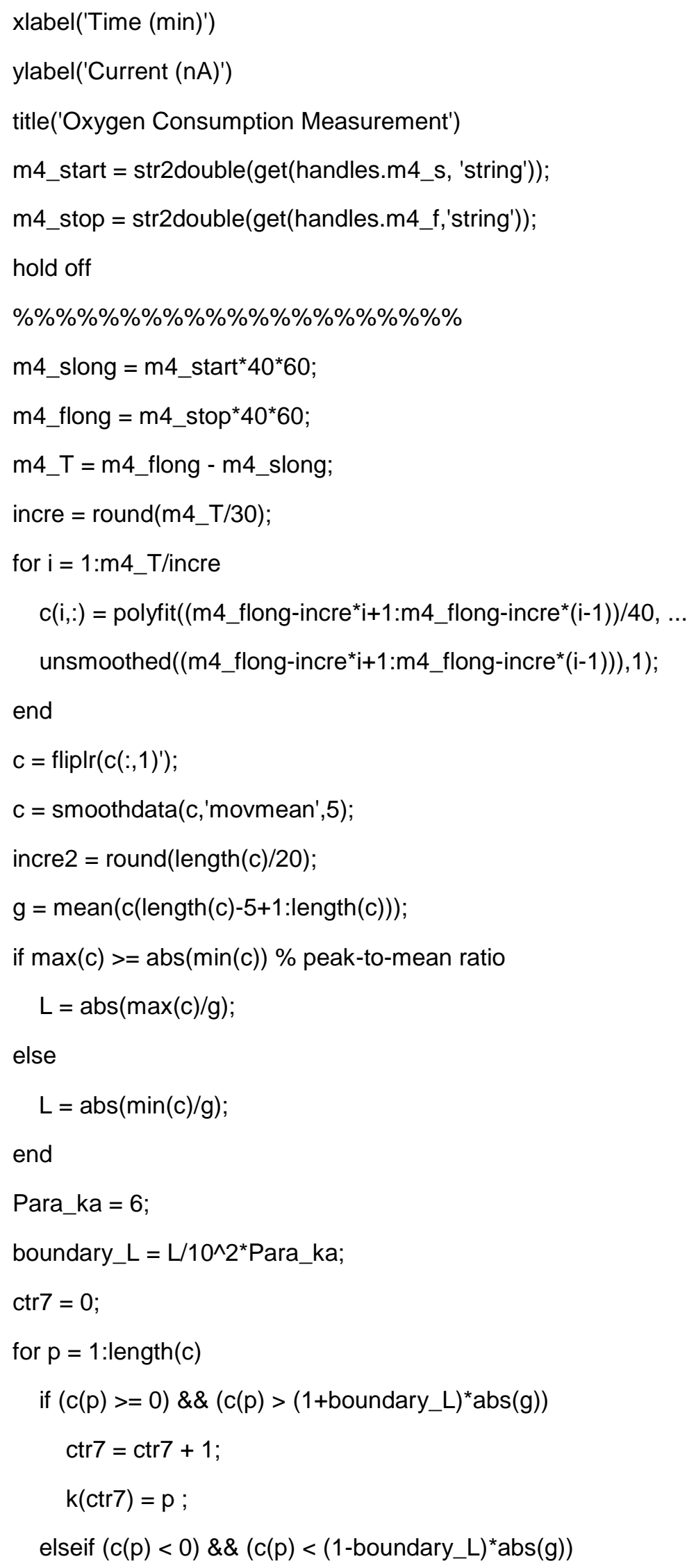




$$
\begin{aligned}
& \operatorname{ctr} 7=\operatorname{ctr} 7+1 \\
& \mathrm{k}(\operatorname{ctr} 7)=\mathrm{p} ;
\end{aligned}
$$

end

end

k_cal $=\mathrm{k} ; \%{ }^{*}$ basal_s * 60 * 40 ;

k_max $=\max \left(\mathrm{k} \_\right.$cal $)$;

$q=\left(k \_m a x-0.5\right) *$ incre $/ 60 / 40+m 4 \_s t a r t ;$

a11 = line([q q], get(gca, 'ylim'), 'Color','m');

$\% \% \% \% \% \% \% \% \% \% \% \% \% \% \% \%$ line for showing the reading period

a1 = line([m4_start m4_start], get(gca, 'ylim'), 'Color','r');

a2 = line([m4_stop m4_stop], get(gca, 'ylim'),'Color','r');

if data $2=0$

else

legend('raw data','smoothed data')

end

\%\%\%\%\%\%\%\%\%\%\%\%\%\%\%\%\%\%\%\%\%\%\%\%\%\% linear curve fitting

$\%$ [slope, intercept] $=$ polyfit

data_m4 $=$ smoothdata $\left(\right.$ unsmoothed $\left(q^{*} 60 * 40: m 4 \_s t o p^{*} 60 * 40\right)$, 'gaussian',50);

coefficients_m4 $=$ polyfit $\left(\right.$ linspace $\left(q^{*} 60, m 4 \_s t o p * 60, \ldots\right.$

length(data_m4)), data_m4, 1);

\%\%\%\%\%\%\%\%\%\%\%\%\%\%\%\%\%\%\%\%\%\%\%\%\%\%\%\%\%\% Conversion

cell_height $=$ str2double $($ get(handles.cell_height, 'string'));

slope_m4 = coefficients_m4(1);

dl_dC = 1.632113498397532e-09;

if (cell_height $>=80) \& \&$ (cell_height $<=300$ )

OC_m4 = slope_m4/dl_dC* $\left(0.25^{*} 0.25^{*} 3.14159\right)^{*}$ cell_height $/ 1000^{*} 10^{\wedge}(-6)$;

else

$\mathrm{OC} \_\mathrm{m} 4=$ slope $\_\mathrm{m} 4 / \mathrm{dl} \_\mathrm{dC} \mathrm{C}^{*}\left(0.25^{\star} 0.25^{\star} 3.14159\right)^{*} 0.150^{*} 10^{\wedge}(-6)$;

disp('Please set the diameter of the cell (Default: 150 um)')

end

set(handles.m4_result, 'string', OC_m4); \% show the result on the GUI disp('Analysis of Measurement 4 is done...') 
disp(['Measurement taken from ',num2str(q),' min to ', num2str(m4_stop), ' min.'])

function m2_n_Callback(hObject, eventdata, handles)

$\%$ hObject handle to edit11 (see GCBO)

$\%$ eventdata reserved - to be defined in a future version of MATLAB

$\%$ handles structure with handles and user data (see GUIDATA)

$\%$ Hints: get(hObject,'String') returns contents of edit11 as text

$\% \quad$ str2double(get(hObject,'String')) returns contents of edit11 as a double

$\%$--- Executes during object creation, after setting all properties.

function m2_n_CreateFcn(hObject, eventdata, handles)

$\%$ hObject handle to edit11 (see GCBO)

$\%$ eventdata reserved - to be defined in a future version of MATLAB

$\%$ handles empty - handles not created until after all CreateFcns called

\% Hint: edit controls usually have a white background on Windows.

$\% \quad$ See ISPC and COMPUTER.

if ispc \&\& isequal(get(hObject,'BackgroundColor'), get(0,'defaultUicontrolBackgroundColor')) set(hObject,'BackgroundColor','white');

end

function m3_n_Callback(hObject, eventdata, handles)

$\%$ hObject handle to edit11 (see GCBO)

$\%$ eventdata reserved - to be defined in a future version of MATLAB

$\%$ handles structure with handles and user data (see GUIDATA)

\% Hints: get(hObject,'String') returns contents of edit11 as text

$\% \quad \operatorname{str2double(get(hObject,'String'))~returns~contents~of~edit11~as~a~double~}$

$\%$--- Executes during object creation, after setting all properties.

function m3_n_CreateFcn(hObject, eventdata, handles)

$\%$ hObject handle to edit11 (see GCBO)

$\%$ eventdata reserved - to be defined in a future version of MATLAB

$\%$ handles empty - handles not created until after all CreateFcns called

$\%$ Hint: edit controls usually have a white background on Windows.

$\%$ See ISPC and COMPUTER.

if ispc \&\& isequal(get(hObject,'BackgroundColor'), get(0,'defaultUicontrolBackgroundColor')) set(hObject,'BackgroundColor','white'); 
end

function edit11_Callback(hObject, eventdata, handles)

$\%$ hObject handle to edit11 (see GCBO)

$\%$ eventdata reserved - to be defined in a future version of MATLAB

$\%$ handles structure with handles and user data (see GUIDATA)

\% Hints: get(hObject,'String') returns contents of edit11 as text

$\% \quad$ str2double(get(hObject,'String')) returns contents of edit11 as a double

$\%$--- Executes during object creation, after setting all properties.

function edit11_CreateFcn(hObject, eventdata, handles)

$\%$ hObject handle to edit11 (see GCBO)

$\%$ eventdata reserved - to be defined in a future version of MATLAB

$\%$ handles empty - handles not created until after all CreateFcns called

\% Hint: edit controls usually have a white background on Windows.

$\% \quad$ See ISPC and COMPUTER.

if ispc \&\& isequal(get(hObject,'BackgroundColor'), get(0,'defaultUicontrolBackgroundColor')) set(hObject,'BackgroundColor','white');

end

function m5_s_Callback(hObject, eventdata, handles)

$\%$ hObject handle to m5_s (see GCBO)

$\%$ eventdata reserved - to be defined in a future version of MATLAB

$\%$ handles structure with handles and user data (see GUIDATA)

\% Hints: get(hObject,'String') returns contents of m5_s as text

$\% \quad$ str2double(get(hObject,'String')) returns contents of m5_s as a double

$\%$--- Executes during object creation, after setting all properties.

function m5_s_CreateFcn(hObject, eventdata, handles)

\% hObject handle to m5_s (see GCBO)

$\%$ eventdata reserved - to be defined in a future version of MATLAB

$\%$ handles empty - handles not created until after all CreateFcns called

$\%$ Hint: edit controls usually have a white background on Windows.

$\%$ See ISPC and COMPUTER.

if ispc \&\& isequal(get(hObject,'BackgroundColor'), get(0,'defaultUicontrolBackgroundColor')) set(hObject,'BackgroundColor','white'); 
end

function m5_f_Callback(hObject, eventdata, handles)

$\%$ hObject handle to m5_f (see GCBO)

$\%$ eventdata reserved - to be defined in a future version of MATLAB

$\%$ handles structure with handles and user data (see GUIDATA)

\% Hints: get(hObject,'String') returns contents of m5_f as text

$\% \quad$ str2double(get(hObject,'String')) returns contents of m5_f as a double

$\%$--- Executes during object creation, after setting all properties.

function m5_f_CreateFcn(hObject, eventdata, handles)

$\%$ hObject handle to m5_f (see GCBO)

$\%$ eventdata reserved - to be defined in a future version of MATLAB

$\%$ handles empty - handles not created until after all CreateFcns called

\% Hint: edit controls usually have a white background on Windows.

$\% \quad$ See ISPC and COMPUTER.

if ispc \&\& isequal(get(hObject,'BackgroundColor'), get(0,'defaultUicontrolBackgroundColor')) set(hObject,'BackgroundColor','white');

end

$\%$--- Executes on button press in m5_run.

function m5_run_Callback(hObject, eventdata, handles)

global data data2 unsmoothed cell_height

if data $2==0$

plot(linspace $(0$,length(data) $/ 60 / 40$,length(data))',data...

,'LineWidth',1.5)

else

plot(linspace( 0 ,length(data2)/60/40, length(data2))',...

data2,'LineWidth',1.5)

hold on

plot(linspace(0,length(data2)/60/40,length(data2))',data)

end

grid on

xlabel('Time (min)')

ylabel('Current $\left.(\mathrm{nA})^{\prime}\right)$ 


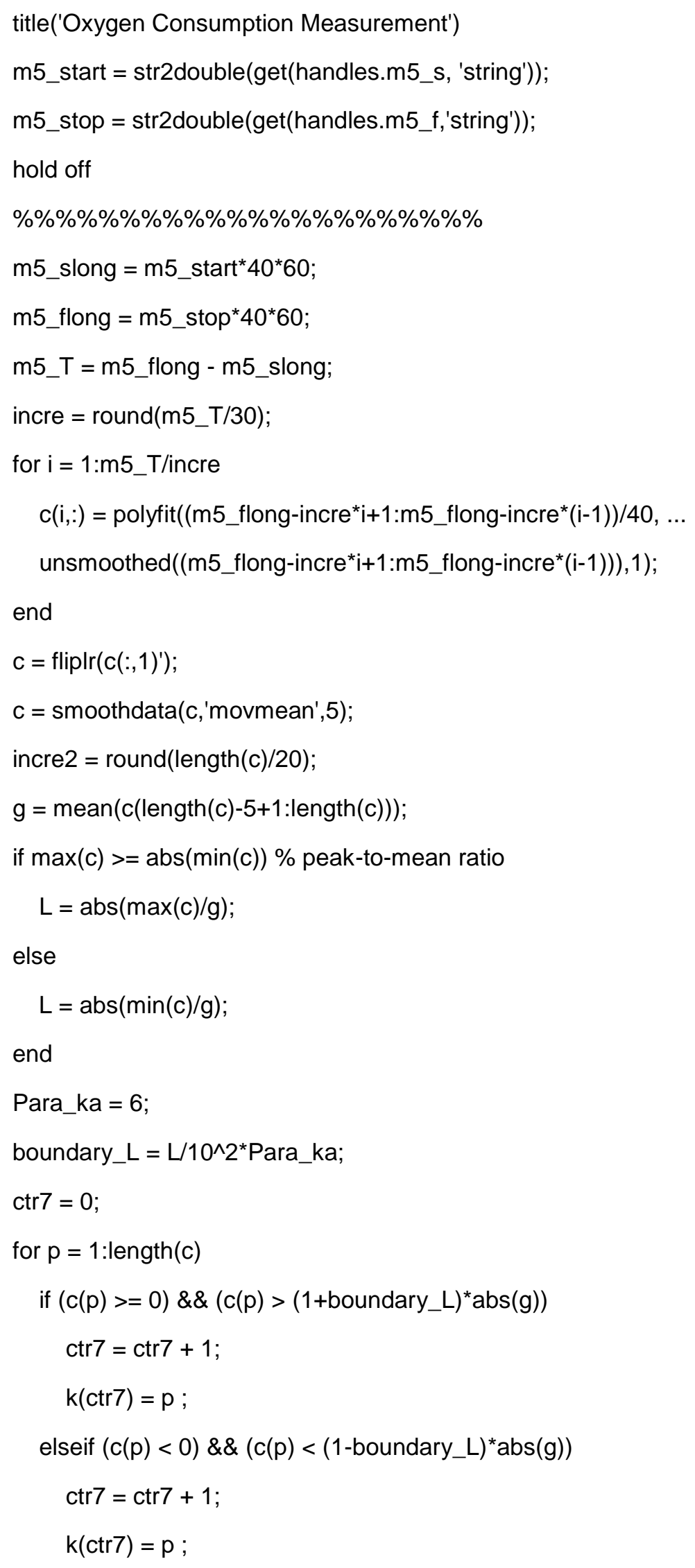


end

end

k_cal $=\mathrm{k} ; \%{ }^{*}$ basal_s ${ }^{*} 60$ * 40 ;

k_max $=\max \left(\mathrm{k} \_\right.$cal $)$;

$q=\left(k \_\right.$max-0.5) * incre /60/40 + m5_start;

a11 = line([q q], get(gca, 'ylim'), 'Color','m');

$\% \% \% \% \% \% \% \% \% \% \% \% \% \% \% \%$ line for showing the reading period

a1 = line([m5_start m5_start], get(gca, 'ylim'), 'Color','r');

a2 = line([m5_stop m5_stop], get(gca, 'ylim'),'Color','r');

\%\%\%\%\%\%\%\%\%\%\%\%\%\%\%\%\%\%\%\%\%\%\%\%\%\%\%\%\%\%\%\%\%\%\%\%\%\%\%\%\%\%\%\%\%\%\%\%\%\%\%\%

if data2 $==0$

else

legend('raw data','smoothed data')

end

$\% \% \% \% \% \% \% \% \% \% \% \% \% \% \% \% \% \% \% \% \% \% \% \% \%$ linear curve fitting

$\%$ [slope, intercept $]=$ polyfit

data_m5 = smoothdata(unsmoothed(q*60*40:m5_stop*60*40),'gaussian',50);

coefficients_m5 $=$ polyfit(linspace $\left(q^{*} 60, m 5 \_s t o p * 60, \ldots\right.$

length(data_m5)), data_m5, 1);

\%\%\%\%\%\%\%\%\%\%\%\%\%\%\%\%\%\%\%\%\%\%\%\%\%\%\%\%\%\% Conversion

cell_height $=$ str2double $($ get $($ handles.cell_height, 'string'));

slope_m5 = coefficients_m5(1);

dl_dC = 1.632113498397532e-09;

if (cell_height $>=80) \& \&$ (cell_height $<=300$ )

$\mathrm{OC} \_\mathrm{m} 5=$ slope_m5/dl_dC* $\left(0.25^{*} 0.25^{*} 3.14159\right)^{*}$ cell_height $/ 1000^{*} 10^{\wedge}(-6)$;

else

OC_m5 $=$ slope_m5/dl_dC ${ }^{*}\left(0.25^{*} 0.25^{\star} 3.14159\right)^{\star} 0.150^{*} 10^{\wedge}(-6) ;$

disp('Please set the diameter of the cell (Default: 150 um)')

end

set(handles.m5_result, 'string', OC_m5); \% show the result on the GUI

disp('Analysis of Measurement 5 is done...')

disp(['Measurement taken from ',num2str(q),' min to ', num2str(m5_stop), ' min.']) 
function edit14_Callback(hObject, eventdata, handles)

$\%$ hObject handle to edit14 (see GCBO)

$\%$ eventdata reserved - to be defined in a future version of MATLAB

$\%$ handles structure with handles and user data (see GUIDATA)

\% Hints: get(hObject,'String') returns contents of edit14 as text

$\% \quad$ str2double(get(hObject,'String')) returns contents of edit14 as a double

$\%$--- Executes during object creation, after setting all properties.

function edit14_CreateFcn(hObject, eventdata, handles)

\% hObject handle to edit14 (see GCBO)

$\%$ eventdata reserved - to be defined in a future version of MATLAB

$\%$ handles empty - handles not created until after all CreateFcns called

$\%$ Hint: edit controls usually have a white background on Windows.

$\% \quad$ See ISPC and COMPUTER.

if ispc \&\& isequal(get(hObject,'BackgroundColor'), get(0,'defaultUicontrolBackgroundColor')) set(hObject,'BackgroundColor','white');

end

$\%$--- Executes on button press in reset_butt.

function reset_butt_Callback(hObject, eventdata, handles)

$\%$ hObject handle to reset_butt (see GCBO)

$\%$ eventdata reserved - to be defined in a future version of MATLAB

$\%$ handles structure with handles and user data (see GUIDATA)

set(handles.Sample_ID,'String', 'Sample ID');

\% set(handles.m2_n,'String', 'Measurement 2');

set(handles.Sensitivity,' Value',6);

set(handles.sen_level,'String', 6.0)

set(handles.cell_height, 'string', 'Diameter');

set(handles.result_basal, 'string', 'Rate');

set(handles.basal_s, 'string', 'Start Time (min)');

set(handles.basal_f, 'string', 'End Time (min)');

set(handles.m2_s, 'string', 'Start Time $(\mathrm{min})$ ');

set(handles.m2_f, 'string', 'End Time (min)');

set(handles.m2_result, 'string', 'Rate'); 


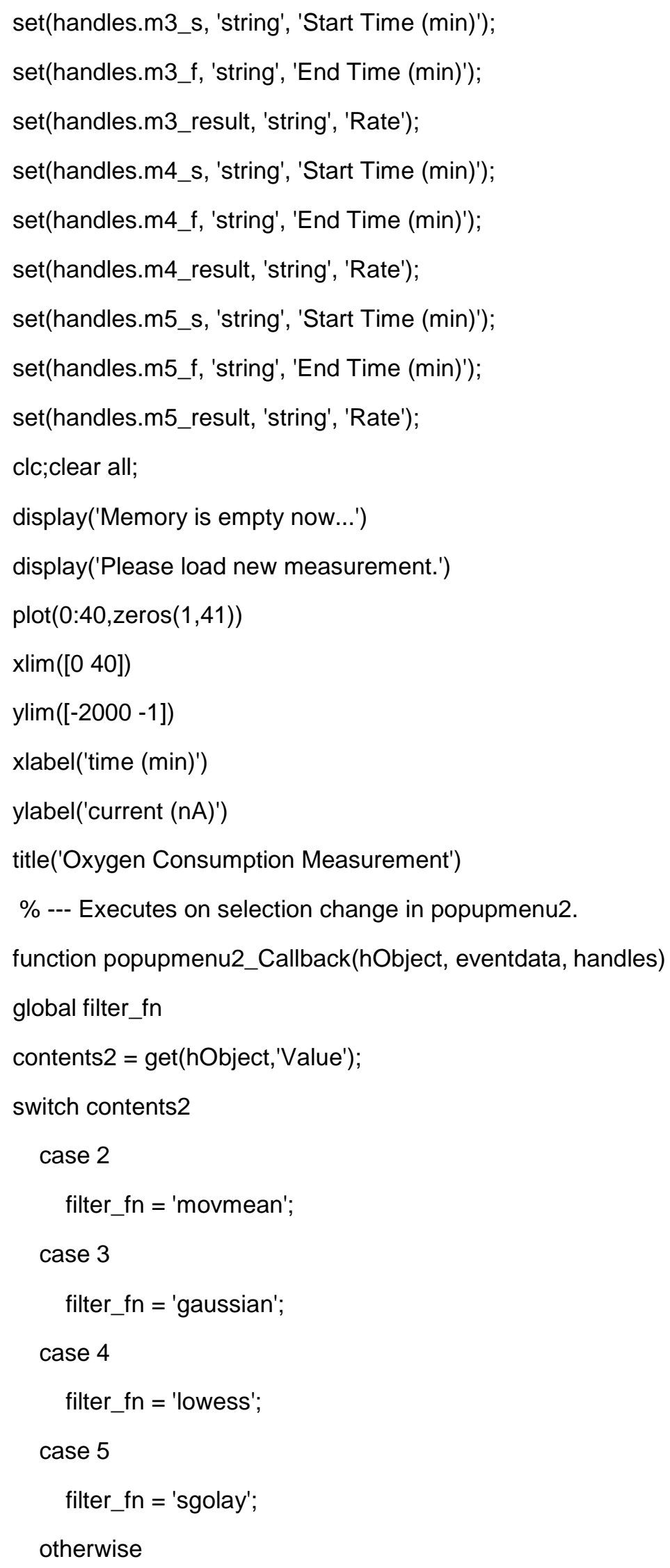


filter_fn = 'movmean';

end

$\%$--- Executes during object creation, after setting all properties.

function popupmenu2_CreateFcn(hObject, eventdata, handles)

$\%$ hObject handle to popupmenu2 (see GCBO)

$\%$ eventdata reserved - to be defined in a future version of MATLAB

$\%$ handles empty - handles not created until after all CreateFcns called

$\%$ Hint: popupmenu controls usually have a white background on Windows.

$\% \quad$ See ISPC and COMPUTER.

if ispc \&\& isequal(get(hObject,'BackgroundColor'), get(0,'defaultUicontrolBackgroundColor'))

set(hObject,'BackgroundColor','white');

end

function filter_num_Callback(hObject, eventdata, handles)

$\%$ hObject handle to filter_num (see GCBO)

$\%$ eventdata reserved - to be defined in a future version of MATLAB

$\%$ handles structure with handles and user data (see GUIDATA)

\% Hints: get(hObject,'String') returns contents of filter_num as text

$\% \quad$ str2double(get(hObject,'String')) returns contents of filter_num as a double

$\%$--- Executes during object creation, after setting all properties.

function filter_num_CreateFcn(hObject, eventdata, handles)

$\%$ hObject handle to filter_num (see GCBO)

$\%$ eventdata reserved - to be defined in a future version of MATLAB

$\%$ handles empty - handles not created until after all CreateFcns called

\% Hint: edit controls usually have a white background on Windows.

$\% \quad$ See ISPC and COMPUTER.

if ispc \&\& isequal(get(hObject,'BackgroundColor'), get(0,'defaultUicontrolBackgroundColor')) set(hObject,'BackgroundColor','white');

end

$\%$--- Executes on button press in filter_butt.

function filter_butt_Callback(hObject, eventdata, handles)

global data data2 filter_fn unsmoothed

data2 = unsmoothed; 


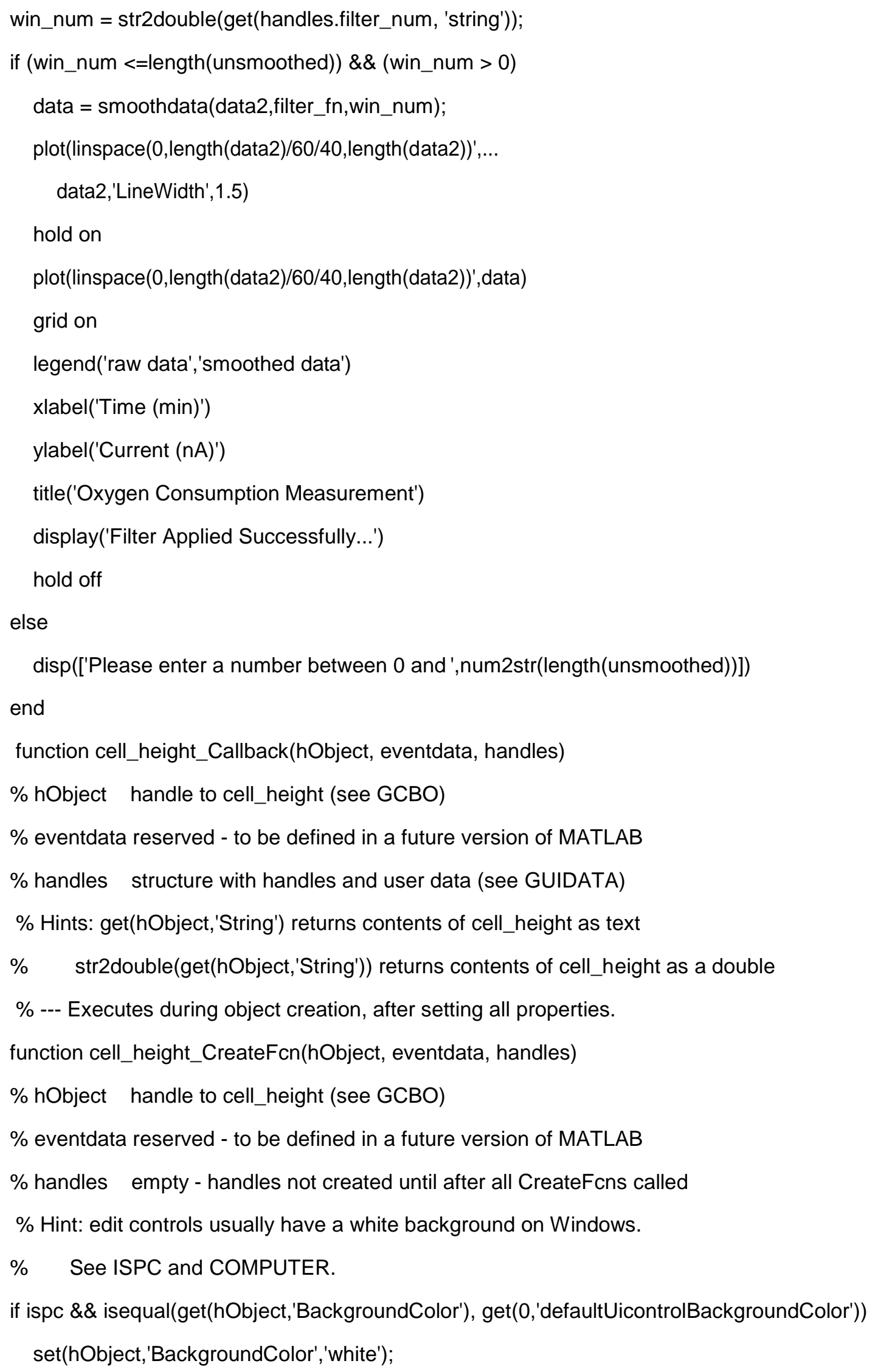


end

$\%$--- Executes on slider movement.

function Sensitivity_Callback(hObject, eventdata, handles)

$\%$ hObject handle to Sensitivity (see GCBO)

$\%$ eventdata reserved - to be defined in a future version of MATLAB

$\%$ handles structure with handles and user data (see GUIDATA)

kk = round(get(hObject,''Value'),1);

set(handles.sen_level,'String', kk);

\% Hints: get(hObject,'Value') returns position of slider

$\% \quad$ get(hObject,'Min') and get(hObject,'Max') to determine range of slider

$\%$--- Executes during object creation, after setting all properties.

function Sensitivity_CreateFcn(hObject, eventdata, handles)

$\%$ hObject handle to Sensitivity (see GCBO)

$\%$ eventdata reserved - to be defined in a future version of MATLAB

$\%$ handles empty - handles not created until after all CreateFcns called

$\%$ Hint: slider controls usually have a light gray background.

if isequal(get(hObject,'BackgroundColor'), get(0,'defaultUicontrolBackgroundColor')) set(hObject,'BackgroundColor',[.9 .9 .9]);

end

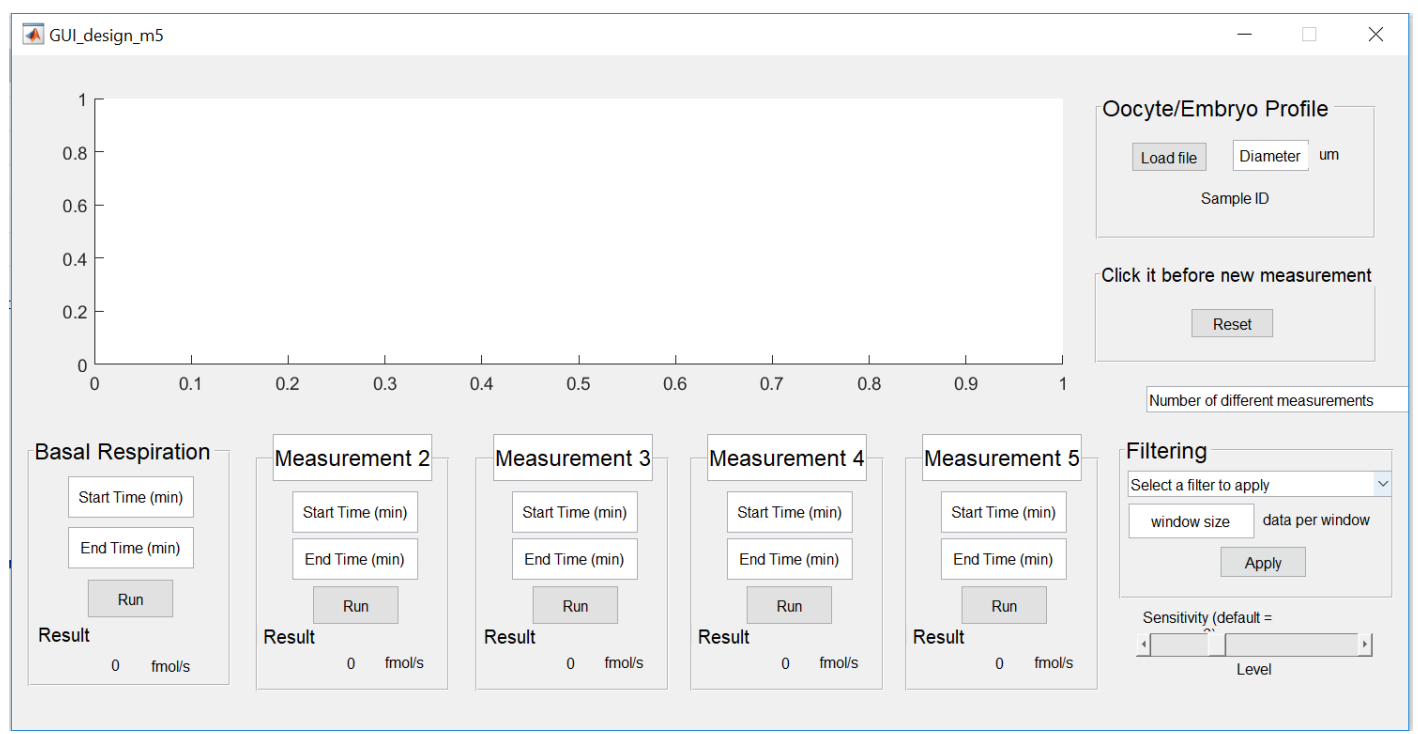

Fig. F.1. Matlab Gui screen 


\section{Appendix G: Publications}

[1] Conference paper: Y. Obeidat and T. Chen, "Characterization of an O2 Sensor Using Microelectrodes". IEEE sensors. 2016, Orlando, FL, Oct. 30 - Nov. 2.

[2] Published journal paper: Obeidat, Y., Evans, A., Tedjo, W., Chicco, A., Carnevale, E., Chen, T. 2018a. Monitoring Oocyte/Embryo Respiration Using Electrochemical-Based Oxygen Sensors, Sensors and amp; Actuators: B. Chemical, https://doi.org/10.1016/j.snb.2018.07.157.

[3] Published journal paper: Obeidat, Y., Catandi, G., Carnevale, E., Chicco, A., DeMann, A., Field, S., Chen, T. 2018b. A Multi-Sensor System for Measuring Bovine Embryo Metabolism, Biosensors and Bioelectronic. DOI: https://doi.org/10.1016/j.bios.2018.09.071.

[4] Submitted journal paper: Obeidat, Y., Cheng, M-H., Catandi, G., Carnevale, E., Chicco, A., DeMann, A., Field, S., Chen, T. 2018c. Understanding Mitochondrial Bioenergetics and the Role of Glycolysis in Equine Embryos, Biosensors and Bioelectronic. 